\title{
Luttinger-liquid physics in wire and dot geometries
}

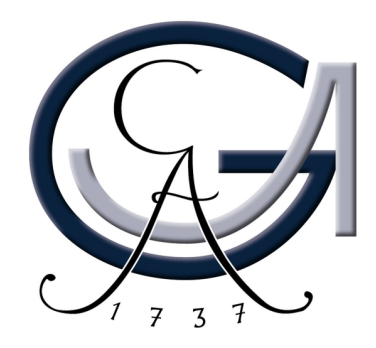

\author{
Dissertation \\ zur Erlangung des \\ mathematisch-naturwissenschaftlichen Doktorgrades \\ „Doctor rerum naturalium“ \\ der Georg-August-Universität zu Göttingen
}

vorgelegt von

\section{Hans Peter Wächter}

aus Hannover

Göttingen 2009 
D7

Referent: Prof. Dr. Kurt Schönhammer

Koreferent: Prof. Dr. Rainer Kree

Tag der mündlichen Prüfung: 16.12.2009 




\section{preface/Vorwort}

Über Universitätsprofessoren stellte Max Weber 1917 fest:1ำ

«Es kann jemand ein ganz hervorragender Gelehrter und ein geradezu entsetzlich schlechter Lehrer sein. Ich erinnere an die Lehrtätigkeit von Männern wie Helmholtz oder wie Ranke. Und das sind nicht etwa seltene Ausnahmen.»

Es mag als besonderer Glücksfall angesehen werden, dass gleich zwei Professoren meine Promotion begleiteten, die ihre exzellenten wissenschaftlichen Fähigkeiten mit guter Lehre zu verbinden wussten. Ich danke von ganzem Herzen Prof. Dr. Kurt Schönhammer für das Angebot, in seiner Arbeitsgruppe ein Promotionsprojekt aufnehmen zu können und für seine Bereitschaft, dieses durch seine physikalische Erfahrung und präzise Art des Denkens zu unterstützen. Nicht minder danke ich Prof. Dr. Volker Meden für seine inspirierende Art, physikalische Problemlösungen voranzutreiben und die Beantwortung unzähliger Fragen. Prof. Dr. Rainer Kree sei gedankt für die Übernahme eines Koreferats zu dieser Arbeit.

Desweiteren danke ich Dipl.-Phys. Ansgar Kalz und Dipl.-Phys. Piet Dargel für die vielen (physikalischen) Diskussionen in unserem Büro und für das Korrekturlesen des Entwurfes der vorliegenden Dissertation. Frau Lepa-Wächter danke ich für wertvolle Hinweise bezüglich guter und richtiger englischer Sprache. Für jeden verbleibenden Rechtschreibfehler und jedes verbleibende grammatikalische Missgeschick bin ich alleine verantwortlich.

Speziell danke ich meinem Vater für das Angebot, zu jeder Zeit beliebige Museen und Ausstellungen zur Zerstreuung aufzusuchen.

Schließlich sei der Deutschen Forschungsgemeinschaft gedankt für die Unterstützung im Rahmen des Sonderforschungsbereiches 602 "Komplexe Strukturen in kondensierter Materie von atomarer bis mesoskopischer Skala".

Göttingen,

Hans Peter Wächter

November 2009

\footnotetext{
${ }^{1}$ Weber M. C. E., Wissenschaft als Beruf (Philipp Reclam jun., Stuttgart, 1995), S. 9
} 



\section{table of contents}

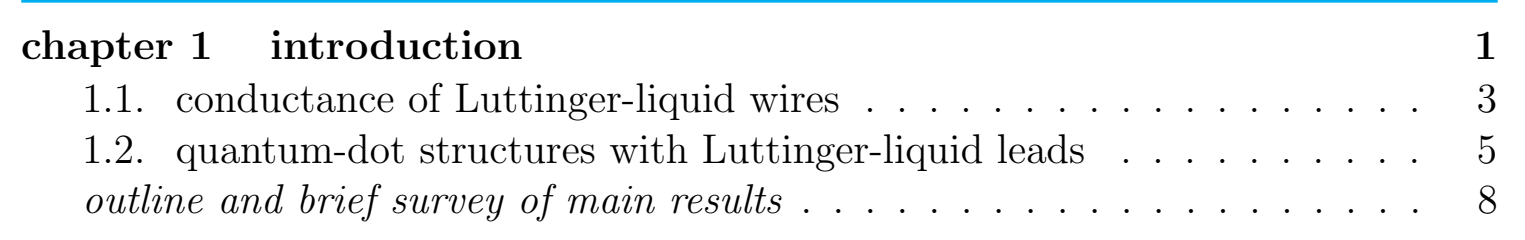

$\begin{array}{lll}\text { chapter } 2 & \text { interacting electrons in one spatial dimension } & 11\end{array}$

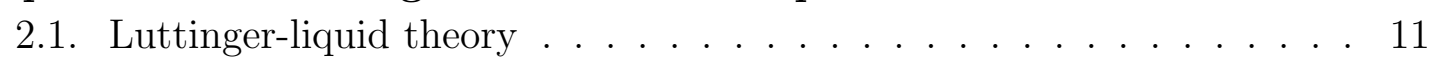

2.2. spinless fermions on a chain . . . . . . . . . . . . . . . 14

$\begin{array}{lll}\text { chapter } 3 & \text { computational methods } & 17\end{array}$

3.1. the functional renormalization group . . . . . . . . . . . . . . . 17

3.1.1. basics of the one-particle irreducible scheme . . . . . . . . . . 18

3.1.2. adaption for one-dimensional lattice systems . . . . . . . . . 20

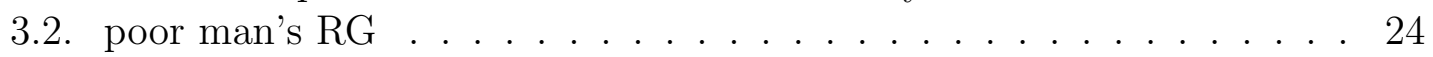

3.3. computing observables . . . . . . . . . . . . . . . . . . . . 25

3.3.1. conductance . . . . . . . . . . . . . . . . . . 25

3.3.2. local spectral function . . . . . . . . . . . . . . . 26

3.3.3. charging . . . . . . . . . . . . . . . . . 27

\begin{tabular}{lll}
\cline { 2 - 2 } chapter 4 & conductance of Luttinger-liquid wires & 29
\end{tabular}

side note: length scales in mesoscopic systems . . . . . . . . . . . . . . 31

4.1. quantum wire with arbitrarily coupled one-dimensional leads . . . . . 32

side note: the stub in poor man's RG . . . . . . . . . . . . . . . . . . . 40

4.2. quantum wire with arbitrarily coupled two-dimensional leads . . . . . 41

side note: the role of spin . . . . . . . . . . . . . . . . . . . 49

\begin{tabular}{lll}
\cline { 2 - 3 } chapter 5 & quantum-dot structures with Luttinger-liquid leads & 51
\end{tabular}

5.1. dot structures coupled to Luttinger-liquid leads within the approxi-

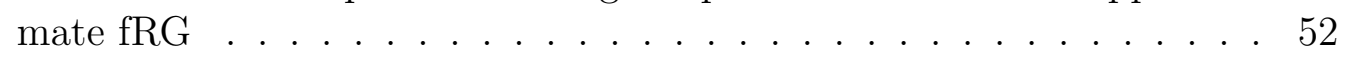

$5.2 . \quad$ single dot with two Luttinger-liquid leads (a) . . . . . . . . . . . . . . . 54

5.2 .1$. conductance . . . . . . . . . . . . . . . 54

5.2 .2$. charging . . . . . . . . . . . . . . . . . . 54

side note: the role of $\operatorname{spin} \ldots \ldots$. . . . . . . . . . . . . 59

5.2.3. schematic proposal of experimental setup . . . . . . . . . . . . 59

side note: charging of a side-coupled dot (b) and end-coupled dot (c) . . . 60

5.3. parallel double dot with two Luttinger-liquid leads $(\mathrm{d})$. . . . . . . . 62

side note: dot-lead interaction within the approximate fRG . . . . . . . . . 67 
$\begin{array}{lll}\text { chapter } 6 & \mathbf{7 1}\end{array}$

6.1. conductance of Luttinger-liquid wires . . . . . . . . . . . . . . . . 71

6.2. quantum-dot structures with Luttinger-liquid leads . . . . . . . . . . 72

appendices i i

appendix A normalization of physical units iii

appendix B projection formalism $\quad$ v

appendix $\mathrm{C}$ the inversion algorithms vii

C.1. tridiagonal matrices ................... . vii

C.2. algorithm for complex geometries . . . . . . . . . . . . . . . . viii

C.2.1. subdiagonal, superdiagonal and diagonal . . . . . . . . . . . . ix

C.2.2. the block $(2,4) \ldots \ldots \ldots$. . . . . . . . . . xi

appendix D the models for the leads $\quad$ Xv

D.1. the semi-infinite hopping chain . . . . . . . . . . . . . . xv

D.2. Green function of the one-dimensional chain . . . . . . . . . . . . . . xvi

D.3. the semi-infinite hopping stripe . . . . . . . . . . . . . . . xvi

D.4. Green function of the two-dimensional stripe . . . . . . . . . . . . . xvii

appendix $\mathrm{E}$ explicit calculation of $\mathcal{T}^{(i)}$ and $\mathcal{T}^{(i i)} \quad$ xix

E.1. computing $\mathcal{T}^{(i)}$ : single-particle scattering theory . . . . . . . . . xix

E.2. computing $\mathcal{T}^{(i i)}$ : vanishing corrections to the Landauer-Büttiker formula ..................... . . . . . . . . . .

appendix $\mathrm{F}$ details of the perturbation theory for the single $\operatorname{dot} \quad \mathrm{xxv}$

F.1. perturbative calculation of $\Sigma^{\mathrm{dot}}(z) \ldots \ldots \ldots \ldots$. . . . . . . . . . . . . . . . .

F.2. charging and width . . . . . . . . . . . . . . . . xxvi

appendix G parallel double quantum dot with non-interacting leads xxix

list of references $\quad$ xxxiii 


\section{introduction}

In his pioneering 1950-paper on interacting fermions in one spatial dimension [Tom50], Sin-itiro Tomonaga wrote:

$\ll$ A mathematically closed and clear-cut presentation of the theory is achieved, however, at the expense of physical usefulness (sic!), because, thus far, the author has succeeded only in giving a complete formulation for a one-dimensional assembly of particles.» [Tom50, p. 546]

Some 25 years after this harsh judgment, H. Keller (professor for inorganic chemistry in Heidelberg at that time) stated on the occasion of a NATO Advanced Study Institute on chemistry and physics of one-dimensional metals (held in Bolzano, 1976):

«Theoretical and experimental work on solids with low-dimensional cooperative phenomena has mushroomed in the last few years, and it seems to be quite fashionable to contribute to this field, especially to the problem of one-dimensional metals.» [Kel76, p. v]

This shows, that one-dimensional physics had by then developed from an elaborate theoretical playground to a full-fledged field of broad physical interest. Up to the present day, this interest stimulates much scientific effort both from the side of theory and from the side of experiment. Put in a somewhat broader perspective, the field of physics at the nanoscale is one of the dominant issues of solid state physics over the last 20 years [DHL07] 1] The experimental control and theoretical understanding of the physical properties of nanostructures is a mandatory prerequisite for successful implementation of technical applications [Hei03, DHL07, NB09].

The problem of (strong) interactions in one-dimensional many-fermion systems ${ }^{2}$ received particular attention [BD04, Gia04]. The breakdown of Fermi-liquid theory, which generically describes the properties of higher ${ }^{3}$-dimensional metals [NO98, GV05, led to the distinct paradigm of Luttinger liquids [Voi94, Sch03], describing the low-energy behavior of a wide range of one-dimensional systems in terms of a few parameters, the Luttinger-liquid parameters, cf. chapter 2, Two important characteristics of Luttinger-liquid behavior, contrasting it to Fermi-liquid behavior, are e. g. the change of the elementary excitations from fermionic quasi-particles to bosonic collective modes with distinct propagation velocities (leading to the notion

\footnotetext{
${ }^{1}$ To any literature cited in the introductory paragraphs, the abbreviation 'e. g.' should be added, as mostly books and reviews are listed, which admittedly may have equitable substitutes.

${ }^{2}$ Thus, exactly the field Tomonaga decisively contributed to while denying it any physical usefulness.

3'Higher' means 'more than one' in this thesis.
} 
of spin-charge separation) and the appearance of anomalous scaling dimensions in the correlation functions, which lead to a power-law behavior of a variety of observables with interaction-dependent exponents Voi94, Sch03. Concerning transport, the impact of interaction and impurities changes drastically in one dimension. Even a single, arbitrary small barrier in a Luttinger liquid leads to vanishing conductance in the low-energy limit [KF92b].

Furthermore, the activity in the field has stimulated the (original or further) development of some formidable calculatory tools, like the Bethe-ansatz [Tak99] or bosonization [LM66, Sch97, vDS98]. After the refinement of the renormalization group (RG) idea [WK74, Wil75, Gol93], and its successful application to onedimensional fermionic systems Sól79, Sha94, several variants, e. g. the densitymatrix-renormalization group (DMRG) [Whi92, Sch05], an adaption of the poor man's RG [MYG93, AW09] and particularly an adaption of the functional renormalization group (fRG) [SH01, Ens05, And06], were developed, which constitute current state-of-art methods.

Experimentally a variety of predictions from Luttinger-liquid theory, like the power-law scaling of certain observables, should be detectable. Various systems with one-dimensional character, like single-channel wires in semiconductor heterostructures, highly anisotropic conductors, chains of gold atoms and carbon nanotubes, have been identified and/or fabricated and spectroscopic as well as transport measurements have been performed. ${ }^{4}$ Particularly strong indications of Luttinger-liquid physics were presented in CPW96, CPW98 and [Cha03, p. 1475 et seq.] for electron tunneling into a quantum Hall edge and in $\mathrm{GDO}^{+} 02$, GAD03 for photoemission spectroscopy on the lithium "purple bronze" $\left(\mathrm{Li}_{0.9} \mathrm{Mo}_{6} \mathrm{O}_{17}\right)$.

However, despite the well advanced theory and theoretical methods and astonishingly refined experimental techniques, the challenge for both, theory and experiment, to converge in a way that non-ambiguous conclusions can be drawn, remains Sch03.

The present thesis shall contribute to meet this challenge from the side of theory. Firstly, the conductance through a Luttinger liquid is examined, generalizing existing theoretical models by explicitly accounting for the coupling geometries necessarily appearing in the coupling of a finite quantum wire to (higher-dimensional) leads. To this end, the functional renormalization group in an adaption for one-dimensional interacting lattice systems is appropriately extended. This project and the choice of the computational method is further motivated and explained in section 1.1 . conductance of Luttinger-liquid wires.

Secondly, novel power laws in the charging of a single dot coupled to Luttingerliquid leads are identified and used to sketch an experimental scheme particularly well suited to probe Luttinger-liquid predictions. It is explained, how the fRG in the extension for the conductance study above can be used to study arbitrary dot

\footnotetext{
${ }^{4}$ Extensive and well annotated listings of these materials and the experimental techniques can be found in Cha03, Sch03. Although about six years old, these reviews give a thorough and by far not outdated overview over materials and techniques. Not covered is the recent controversial observation of Luttinger-liquid physics in shot noise experiments $\mathrm{WQN}^{+} 07$, KROY07.
} 
structures coupled to Luttinger-liquid leads. An exemplary study on double dots coupled in parallel to the leads is performed. This second part is further motivated and explained in section 1.2: quantum-dot structures with Luttinger-liquid leads.

\section{conductance of Luttinger-liquid wires}

The transport properties of a Luttinger-liquid wire, described by the linear conductance $G$ ([BF04, p. 103]), have intensively been studied theoretically and various methods were developed in order to incorporate the coupling to leads.5 In the context of field theoretical models vDS98, e. g., locally varying Luttinger-liquid parameters [MS95, Pon95, SS95, Mas95, JMS06] (called local Luttinger liquid) and special boundary conditions at the ends of the wire [EG96, TEG02, EH98] (called radiative boundary conditions) were proposed. More recently the leads were modelled by attaching the end of semi-infinite non-interacting hopping chains to the end of the wire (modelled itself as interacting hopping chain) Ogu99, Ogu01, $\mathrm{MAM}^{+} 03$, $\mathrm{EMA}^{+}$05, JMS06, $\mathrm{AEM}^{+}$06b. These studies set up a consistent picture for the conductance. If the leads are coupled perfectly to the wire, the conductance shows the finite value $G_{0}=\frac{1}{2 \pi}$ per channe ${ }^{6}$ and does not depend on the length of the wire and the electron-electron interaction 78 For low-transmittance contacts and vanishing bias voltage $V \ll T$, the conductance shows power-law scaling $G \sim T^{\alpha(K)}$ for $\pi v_{F} / L \ll T \ll B$ with $L$ being the length of the wire and $B$ the (model-specific) band width. For temperatures below $\pi v_{F} / L$, the wire becomes effectively zerodimensional and shows transport characteristics of a quantum dot [NB09, p. 509]. The exponent $\alpha(K)$ only depends on the Luttinger-liquid parameter $K$, cf. chapters 2 and 4

The observation of power-law behavior in $G(T)$ was reported in several experimental studies [THS95, $\mathrm{BCL}^{+99}$, YPBD99, $\mathrm{RWD}^{+}$00, $\mathrm{PdYD00}, \mathrm{GKE}^{+}$04]. The most promising materials are metallic single wall carbon nanotubes (SWNT), cf. Har01, RWD ${ }^{+} 00$, PdYD00, $\mathrm{PTY}^{+} 01, \mathrm{GKE}^{+}$04, CBR07, whose low-energy sector was theoretically argued to be a Luttinger liquid [KBF97, EG97. However, the theoretical description of transport in Luttinger liquids briefly sketched above lacks any geometrical features found in typical experimental setups (like overhanging parts of the leads or the wire, cf. fig. 1.1) as the leads are merely coupled to the end of the wire (either in a microscopic model or by inclusion in effective field theories). Furthermore, due to the one-dimensional character of the leads, no explicit modelling of the contact region is possible.

\footnotetext{
${ }^{5}$ For a recent overview over the field of quantum transport through nanostructures in general, cf. NB09.

${ }^{6}$ The normalization of physical units is defined in appendix $\mathrm{A}$

7'Perfectly' is actually defined by the criterion $G=G_{0}$, cf. also [MS03, EMA ${ }^{+} 05$, JMS06].

${ }^{8}$ The perfect value $K G_{0}$ renormalized by the interaction (parametrized by the Luttinger-liquid parameter $K$ ) is obtained if a an infinite Luttinger liquid without any leads [LP74, AR82, KF92c, $\mathrm{MAM}^{+} 03$ is considered.
} 


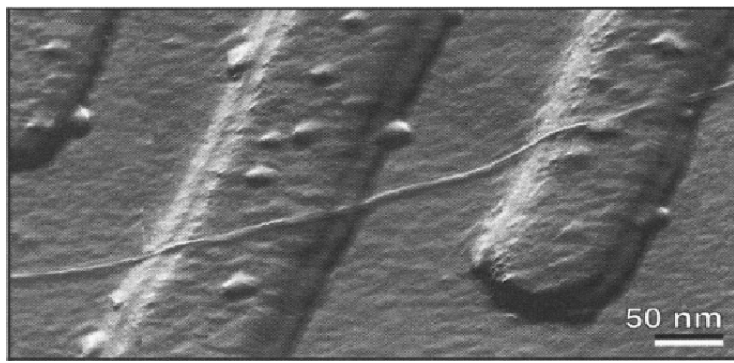

(a)

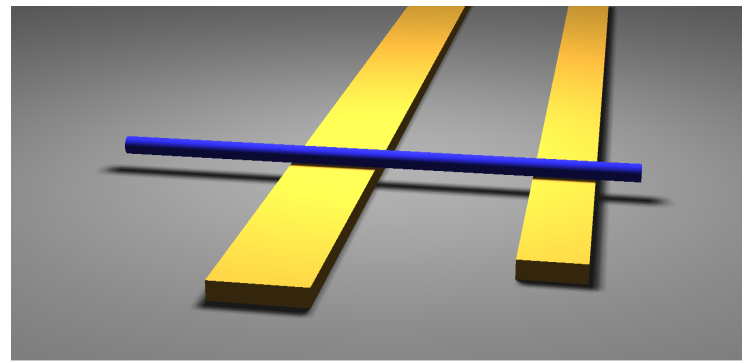

(b)

Figure 1.1: (a) typical experimental setup in experiments on carbon nanotubes [adapted by permission from Macmillan Publishers Ltd: NATURE [TDD ${ }^{+97}$, copyright (1997)]: tube lying on two metal electrodes; (b) artist's view of the simplified situation modelled in this thesis: stripe like Fermi-liquid leads coupled to a one-dimensional Luttinger-liquid conductor, cf. text.

Thus, in this respect, a significant gap between the simplicity of theoretical modelling and the complexity of experimental setups exists. In order to bridge this gap, in this thesis a microscopic model, whose low-energy properties are known to be described by Luttinger-liquid theory, is coupled by two metallic (one-dimensional as well as two-dimensional) leads with Fermi-liquid properties, cf. fig. 1.1 (b), and the conductance of the setup is studied. Specifically, a tight-binding hopping chain of spinles $\mathrm{q}^{9}$ fermions with nearest-neighbour interaction is arbitrarily coupled via hopping terms to non-interacting hopping stripes of variable width. A short summary of the results can be found at the end of this introductory chapter, page 8 .

Microscopic modelling is chosen for two reasons. First, it has proved highly successful in extending Luttinger-liquid theory, originally derived on effective field theories, towards qualitatively more realistic setups, cf. e. g. the discussion of transport above. Second, true ab-initio calculations (cf. Qua08 for a recent review), which would allow for direct comparison with experimental data, fail to include Luttinger-liquid physics (or more generally strong correlations) and are therefore of limited use in this context ${ }^{10}$ cf. also the introductory paragraphs of [PPJL $\left.{ }^{+} 03\right]$.

As computational method of choice to study microscopic models of interacting fermions in the low-energy sector, an adaption of the one-particle irreducible scheme of the fRG ${ }^{11}$ was established in recent years. By comparison to DMRG and exact results ${ }^{12}$ the fRG scheme was shown not only to capture the asymptotic Luttinger-liquid physics qualitatively but also quantitatively to linear order in the

\footnotetext{
${ }^{9}$ The role of spin is discussed in chapter 4

${ }^{10}$ However, recent ab-inito studies on the conductance of non-interacting SWNT emphasized the importance of realistic contact modeling, cf. $\mathrm{PPJL}^{+} 03$, NTC06, NTC08.

${ }^{11} \mathrm{~A}$ detailed account of the development of the $\mathrm{fRG}$ and the several existing schemes is given in [Ens05, chap. 2] and [And06, chap. 3].

${ }^{12}$ As obtained from Bethe ansatz calculations and bosonization in the asymptotic limit Voi94, Gia04.
} 


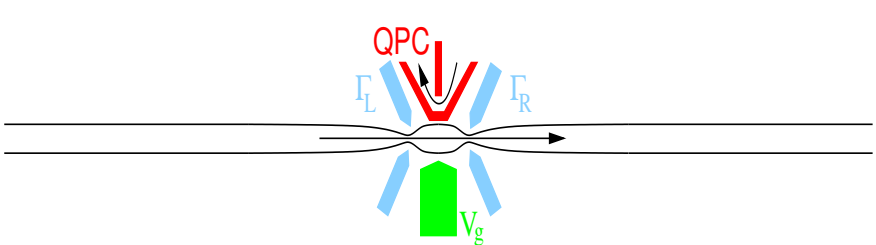

(a)

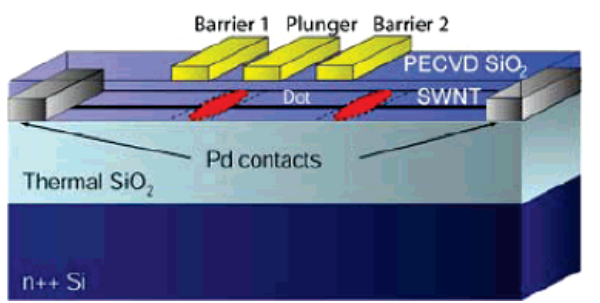

(b)

Figure 1.2: (a) Schematic plot of experimental setup to measure transport through the dot region as well as the charge on the dot. Electrodes define the gate voltage $V_{g}$ and the constrictions $\Gamma_{L}, \Gamma_{R}$. The charge on the dot is measured by a quantum point contact (QPC); (b) state-of-art experimental setup realizing SWNT based quantum dots controlled by electrostatic gates [adapted with permission from [BGM ${ }^{+}$05], copyright 2005 American Chemical Society].

interaction, cf. the detailed studies on impurities in Luttinger liquids [MMSS02a, MMSS02b, $\mathrm{AEM}^{+} 04$ ], on Y-junctions of Luttinger liquids [BTSMS05a, BTSMS05b] and particularly on conductance through end-coupled wires $\left[\mathrm{MAM}^{+} 03, \mathrm{EMA}^{+} 05\right]$. The unique feature is, that hopping chains of up to $10^{7}$ sites can be treated numerically ${ }^{13}$ allowing to study the complete energy-range down to the asymptotic low-energy (Luttinger-liquid) physics, which can actually be reached. This property relies on the usage of a highly efficient inversion algorithm for tridiagonal matrices, scaling linearly with the matrix dimension (i. e. the number of lattice sites in the chain), cf. [Ens05, app. B] and chapter 3. Part of this thesis is an inversion algorithm for matrices with large triangular blocks connected by smaller full blocks, which permits to treat wires up to $10^{6}$ lattice sites coupled in the complex geometry introduced above, cf. chapter 3 and 4.

\section{quantum-dot structures with Luttinger-liquid leads}

Dot structures coupled to Luttinger-liquid leads in various geometries have received considerable interest ${ }^{14}$ One dominant question is, wether traces of Luttinger-liquid behavior can be found in the observables of the system, like the conductance, the average occupation of the dot (charging) or the local spectral function of the dot.

In the case of a single level (quantum dot) coupled to two Luttinger-liquid leads, the conductance is known to show various power laws in various parameter regimes ${ }^{15}$ In chapter 5 novel Luttinger-liquid power laws are identified in the charging of

\footnotetext{
${ }^{13}$ This is a major advantage over DMRG, which becomes infeasible for system sizes exceeding $\sim 10^{3}$, e. g. Sch05 for a review and e. g. $\left[\mathrm{AEM}^{+} 04\right]$ for a comparison.

${ }^{14}$ There is no current review available, but SI97, Fur98, RS04, EMA ${ }^{+}$05, SWGB05, AEM06a, WMS07a, LYY08, GWB09, Kaw09 and their references give a complete overview.

${ }^{15}$ Compare with chapter 5 and the works cited there.
} 
a quantum dot in this geometry. The calculations are done using perturbation theory in the dot-Luttinger-liquid coupling and complemented by an fRG study. The charging shows distinct power laws as function of different external parameters for various interaction strengths, cf. chapter 5 for a detailed account.

Besides the theoretical interest, the availability of multiple observables showing Luttinger-liquid physics can significantly contribute to the challenge of converging theory and experiment. As mentioned earlier, Luttinger-liquid physics manifests e. g. in the power-law scaling of a variety of observables as functions of external parameters with exponents, which can be expressed in terms of a single interactiondependent parameter $K$, cf. chapter 2. An experiment, which measures the powerlaw scaling of a single observable (like the conductance experiments introduced in the preceding section) and extracts the parameter $K$ is troubled to convincingly exclude any other source than Luttinger-liquid physics for the observed data, since $K$ depends on the details of the probe (e. g. interaction, screening, band structure and filling) and no ab-initio estimates exist ${ }^{16}{ }^{17}$ A way to circumvent this problem would be the measurement of two observables for which Luttinger-liquid theory predicts power-law scaling with different exponents $\alpha_{1}(K), \alpha_{2}(K)$, ideally as function of two different external parameters ${ }^{18}$ If the extraction of $K$ from the two exponents yielded a consistent result, i. e. $K\left(\alpha_{1}\right)=K\left(\alpha_{2}\right)$, strong evidence for Luttinger-liquid physics would be achieved.

In the light of recent progress in the fabrication of highly controllable quantum dots in SWNT [MBM04, $\mathrm{BGM}^{+}$05, JGRHL06, SMB ${ }^{+}$06, SGK09] and the novel power laws identified in the charging, an experimental setup is proposed, which allows for the combined measurement of charging on and conductance through the dot, cf. fig. 1.2 (a). The different characteristic power laws of the two observables could be measured and the Luttinger-liquid parameter of the system independently be extracted from each scaling to conduct an experiment as described above, thus providing a unique way for checking consistency with Luttinger-liquid physics. In fig. 1.2 (b), a state-of-art experimental setup $\left[\mathrm{BGM}^{+} 05\right]$ is shown, which readily contains the major features of the experiment proposed. Details are added in chapter 5.

Apart from the studies of the single-dot system, the inversion algorithm for matrices with large triangular blocks connected by smaller full blocks, introduced in section 1.1 for treating quantum wires with complex coupling geometries, is incorporated in an fRG scheme, which allows to study arbitrary dot structures coupled to Luttinger-liquid leads. The modelling of the leads is done with the same tightbinding hopping chain of spinless fermions used to model the quantum wire in section 1.1. cf. also chapter 2. An exemplary study on a system of parallel double quantum

\footnotetext{
${ }^{16}$ For SWNT some phenomenological estimate exists, cf. KBF97, EG98, or [CBR07, p. 720].

${ }^{17}$ Even if such predictions existed, the input parameters would be hard to determine experimentally and would vary from probe to probe.

${ }^{18} \mathrm{~A}$ step into this direction is the linear conductance measurement across an impurity-free part of an SWNT as well as across a part of the same SWNT containing an impurity, reported in YPBD99.
} 
section 1.2. quantum-dot structures with Luttinger-liquid leads

dots is conducted, explaining the implementation in the fRG scheme in detail and giving a complete survey of the conductance of the system. 


\section{outline and brief survey of main results}

\section{chapter 2: interacting electrons in one spatial dimension}

The notion of a Luttinger liquid is introduced and developed as far as needed in the subsequent chapters. The tight-binding chain used to model Luttingerliquid wires and non-interacting leads is introduced and its properties explained.

\section{chapter 3: computational methods}

The computational methods used in the subsequent chapters to study conductance, charging and local spectral function for dots and wires are introduced. In particular, the fRG is developed in a scheme, which allows to study the complex geometries described in the introduction and its properties are discussed in detail. As complementary approach, the poor man's RG is explained.

\section{chapter 4: conductance of Luttinger-liquid wires}

The inclusion of geometric features in the coupling of leads and wire necessarily introduces overhanging parts of the wire as well as of the leads, which add new length scales to the system. It will be shown, that these length scales set new energy scales and the basic temperature range $\pi v_{F} / L \ll T \ll B$ in which power-law scaling in the conductance can occur, is split into up to five different regimes according to these new energy scales. Each regime can show distinct scaling behavior and is separated from the others by extended crossover regions. The results are found to hold in the case of symmetric widths of the coupling regions and locally homogeneous coupling strengths as well as for arbitrary widths and locally varying coupling strengths.

\section{chapter 5: quantum-dot structures with Luttinger-liquid leads}

The charging of a quantum dot, weakly coupled to two Luttinger-liquid leads, is shown to be a continuous function of the gate voltage for weak interactions, becoming discontinuous for stronger interactions. Luttinger-liquid power laws with distinct interaction-dependent exponents are identified in the charging as function of small gate voltage and in the characteristic width of the charging-vs.-gate-voltage curve as function of the coupling to the leads. With these results, the experimental setup discussed in the introduction is further specified.

The fRG technique to include geometric features in the coupling of leads and wire is used to study arbitrary quantum-dot structures coupled to Luttingerliquid leads. The implementation is exemplified for a system of two quantum dots, coupled in parallel to Luttinger-liquid leads. A study of the conductance and the local spectral functions shows the similarity to the problem of a double barrier in a Luttinger liquid.

chapter 6: concluding remarks

A short summary is given and some future prospects are being discussed. 




\section{interacting electrons in one spatial dimension

\section{summary}

In section 2.1 a brief introduction to the Luttinger-liquid paradigm is given, developing the theory as far as needed in subsequent chapters.

In section 2.2 the lattice models used in subsequent chapters are defined and their basic properties are discussed.

The paradigm of three-dimensional metals, Fermi-liquid theory. 11 introduces the notion of quasi-particles, which upon adiabatically switching on the interaction evolve in a one-to-one correspondence from the bare particle states in the noninteracting (and exactly solvable) Fermi gas. Formally, this implies that the oneparticle Green function of the interacting electron system features a pole with finite residue, cf. [NO98, p. 249 et seq.]. The quasi-particles can be classified by the same quantum numbers as the free particles, however, their physical properties (e. g. mass, compressibility) are renormalized by the interaction. This renormalization can be expressed by a set of parameters, the Landau parameters, which are determined by the two-particle interaction of the quasi-particles [NO98, p. 299 et seq.] [GV05, p. 409 et seq.]. The validity of the approach was also checked within renormalization group studies identifying the Fermi liquid as low-energy fixed point [Sha94, DC96].

\section{\begin{tabular}{l|l} 
Luttinger-liquid theory & 2.1
\end{tabular}}

Luttinger-liquid theory is well covered by a number of review papers and books (each emphasizing a different perspective, though), cf. [Sól79, Sha94, Voi94, vDS98, Sch03, Gia04, GV05. Therefor, no self-contained derivation is given here, but a short introduction of the important points. Some nomenclature in the description of one-dimensional many-fermion systems is given in fig. 2.1 (a) and (c). Part (a) shows the dispersion relation of non-interacting fermions on a lattice (chain) with periodic boundary conditions. Characteristically the dispersion features two Fermi points $\pm k_{F}$ and two branches with $k<0$ and $k>0$, which are used to discriminate the fermions as left- and right-movers respectively. The scattering processes of a two-particle interaction can then be classified according to fig. 2.1.(c).

\footnotetext{
${ }^{1}$ Complete reviews are e. g. given in [NO98, p. 296 et seq.] or GV05, p. 405 et seq.] in modern notation, the original works by Landau in Pin61.
} 


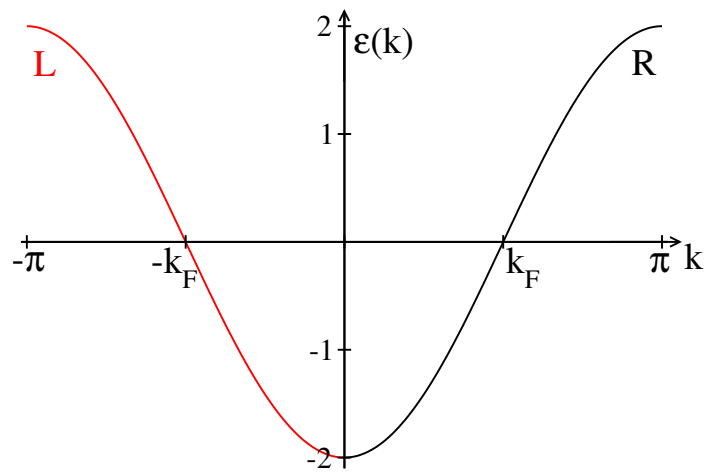

(a)
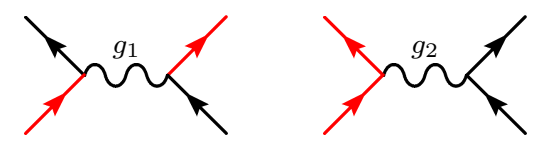

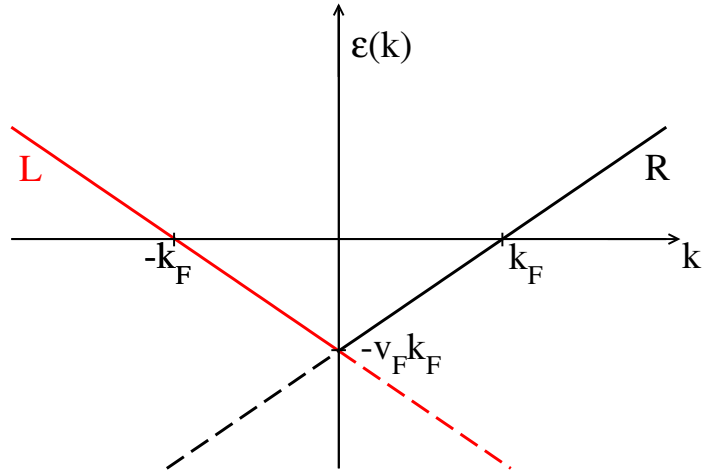

(b)
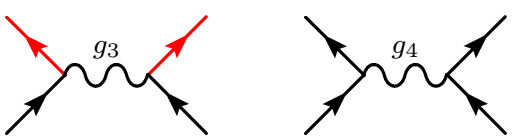

(c)

Figure 2.1: (a) Dispersion $\varepsilon(k)$ of a one-dimensional tight binding Hamiltonian with periodic boundary conditions, $(k<0)$-branch [red]: left-movers $(\mathrm{L}),(k>0)$-branch [black]: right-movers $(\mathrm{R})$; (b) Linear dispersion of the Luttinger model, $(k<0)$-branch [red]: leftmovers $(\mathrm{L}),(k>0)$-branch [black]: right-movers $(\mathrm{R})$, dashed lines: filled Dirac sea. The scale of the y-axis differs from the y-axis in (a). (c) Scattering processes of two-particle interaction [red lines: left-movers; black lines: right-movers]: $g_{2}, g_{4}$ (forward scattering), $g_{3}$ (Umklapp scattering), $g_{1}$ (backward scattering)

Processes with momentum transfer $\approx 2 k_{F}$ (backward scattering) are characterized by the interaction strength $g_{1}$, processes with small momentum transfer (forward scattering) by $g_{2}$ (inter-branch) and $g_{4}$ (intra-branch), processes with momentum transfer $\approx 4 k_{F}$ (Umklapp processes) by $g_{3}$. Including spin leads to the further discrimination $g_{i, \gamma}$ with $i=1, \ldots, 4$ and $\gamma=\perp, \|$ for scattering with parallel (\|) and opposite $(\perp)$ spin, respectively, cf. [Sól79, p. 206].

The $g_{2, \gamma}$ scattering process introduces logarithmic divergences in the expansion of the self-energy, which lead to a vanishing residue at the Fermi surface. Thus, there are no fermionic quasi-particles in contrast to the Fermi liquid picture. Resummed, these divergences lead to the characteristic power laws in the correlation functions. The $g_{4, \gamma}$ scattering process alone generates a branch cut in the one-particle Green function for the model including spin, which leads to the so called spin-charge separation. Due to these forward scattering processes the quasi-particle picture breaks down and Fermi-liquid theory is not applicable, cf. [Voi94, p. 987 et seq.].

It was conjectured [Hal80, Hal81a, Hal81b], that the generic low-energy behavior of gapless one-dimensional systems can instead be described in terms of a model proposed by Tomonaga [Tom50] and Luttinger [Lut63] ? $^{2}$ which starts from a linear

\footnotetext{
${ }^{2}$ Simply called Luttinger liquid in this thesis.
} 
dispersion as illustrated in fig. 2.1. (b) 3 and only assumes forward scattering processes $g_{2, \gamma}$ and $g_{4, \gamma}$ in the two-particle interactions. Deviations from the assumption of a strictly linear dispersion, which generically appear in the expansion of the dispersion around the Fermi points in general models, were shown not to substantially alter the low-energy physics of the Luttinger model [Hal81b, p. 2604] and [Voi94, p. 1030].4 Backscattering $g_{1, \gamma}$ can also be expected to appear in more general models, however a renormalization group analysis shows that if $g_{1, \perp}$ is positive and initially not too strong, $g_{1, \perp}=0$ is a stable fixed point and backscattering merely renormalizes the forward scattering strengths, cf. [Sól79] for details.5 Umklapp scattering $g_{3, \gamma}$ can only be a low-energy process, if the momentum transfer $4 k_{F}$ is a reciprocal lattice vector (i. e. in a lattice model with appropriate filling). If sufficiently strong initially, it does not renormalize to zero but drives the system away from the line of Luttinger-liquid fixed points [Hal81b, Sól79]. An example is the charge density wave, which appears at half-filling for $U / t>2$ in the model of spinless fermions on a chain, cf. next section.

The Luttinger model is exactly solvable, e. g. by bosonization [ML65, Hal81b, Sch97, and its low-energy physics is fully described by two parameters $K_{\nu}, v_{\nu}$ for the spin $(\nu=s)$ and charge $(\nu=c)$ degree of freedom, related to the original parameters over $\left(v_{F}\right.$ : Fermi velocity)

$$
\begin{gathered}
K_{\nu}=\sqrt{\frac{\pi v_{F}+g_{4 \nu}-g_{2 \nu}}{\pi v_{F}+g_{4 \nu}+g_{2 \nu}}} \\
v_{\nu}=\sqrt{\left(v_{F}+\frac{g_{4 \nu}}{\pi}\right)^{2}-\left(\frac{g_{2 \nu}}{\pi}\right)^{2}}
\end{gathered}
$$

and obeying

$$
v_{N \nu} v_{J \nu}=v_{\nu}^{2} \quad, v_{N \nu}=v_{\nu} / K_{\nu} \quad, v_{J \nu}=v_{\nu} K_{\nu} \quad\left(\operatorname{thus} K_{\nu}=\sqrt{v_{J \nu} / v_{N \nu}}\right)
$$

with $g_{2 / 4, \rho}=\frac{1}{2}\left(g_{2 / 4, \|}+g_{2 / 4, \perp}\right)$ and $g_{2 / 4, \sigma}=\frac{1}{2}\left(g_{2 / 4, \|}-g_{2 / 4, \perp}\right)$, [Voi94, p. 998 et seq.]. The $K_{\nu}$ describe the interactions among the degree of freedom $\nu\left(0<K_{\nu}<1\right.$ for repulsive $K_{\nu}>1$ for attractive and $K_{\nu}=1$ for no interaction), $v_{\nu}$ describes the Fermi velocity of the collective excitations. The spinless case is obtained by dropping the spin label $\nu=s$ and for notational simplicity replacing $\left\{K_{c}, v_{N c}, v_{K c}, v_{c}\right\} \rightarrow$ $\left\{K, v_{N}, v_{J}, v\right\}$.

The correlation functions of this model follow power laws in time and space (or frequency and k-space) with exponents depending on the parameters $K_{\nu}$, cf. the

\footnotetext{
${ }^{3}$ The filled Dirac sea of negative energy states constitutes the difference between the approach of Tomonaga and Luttinger: While Tomonaga worked with cutoffs around $\pm k_{F}$, Luttinger extended the branches of the dispersion to infinity, thereby adding a sea of states with negative energy, cf. e. g. Sch97.

${ }^{4}$ This holds for static properties in the low-energy limit, however, to properly describe the dynamic properties a distinct low-energy theory, explicitly taking into account non-linearities, was recently proposed IG09b, IG09a.

${ }^{5}$ This analysis was only recently extended to the case of a system with an open boundary GM09.
} 
reviews Voi94, vDS98. Of particular interest in subsequent chapters will be the local spectral function $\rho_{x}(\omega)$, which follows the power law, cf. [MS92, Voi94, Med96]

$$
\rho_{x=0}(\omega) \sim|\omega|^{\alpha_{\text {bulk }}\left(K_{c}, K_{s}\right)}
$$

with $\alpha_{\text {bulk }}=\frac{1}{4} \sum_{\nu=c, s}\left(K_{\nu}+1 / K_{\nu}-2\right)$. In the case of spin-rotational invariant interaction $\left(K_{s}=1\right) \alpha_{\text {bulk }}=\frac{1}{4}\left(K_{c}+1 / K_{c}-2\right)$ and in the spinless case $\left(K_{s}=K_{c} \rightarrow\right.$ $K) \alpha_{\text {bulk }}=\frac{1}{2}(K+1 / K-2)$. If the system is semi-infinite with an open boundary at $(x=0)$, the exponent in 2.2 reads $\alpha_{\text {end }}=\frac{1}{2} \sum_{\nu=c, s}\left(1 / K_{\nu}-1\right)$, in the spinrotational invariant case $\alpha_{\text {end }}=\frac{1}{2}\left(1 / K_{c}-1\right)$ and $\alpha_{\text {end }}=1 / K-1$ in the spinless case [FG95, $\mathrm{MMS}^{+}$00]. In the presence of a low-energy cutoff (e. g. finite temperature $T$ or system length $L)$, eq. $(2.2)$ reads $\rho_{x=0}(\omega, N, L) \sim \max \left(|\omega|, T, v_{F} / L\right)^{\alpha_{\text {bulk }}\left(K_{c}, K_{s}\right)}$.

Upon validity of Haldane's conjecture, the knowledge of the Luttinger-liquid parameters $\left(K_{\nu}, v_{\nu}\right)$ is thus sufficient to fully describe the low-energy properties of the one-dimensional system at hand. This is expressed by the notion Luttinger-liquid theory ${ }^{6}$ as generic low-energy theory for the correlated one-dimensional electron system.

\section{spinless fermions on a chain}

Consider a one-dimensional tight-binding chain of spinless fermions with nearestneighbour hopping and nearest-neighbour interaction, cf. fig. 2.2. The one-particle part of its Hamiltonian reads $7^{7}$

$$
h^{\text {wire }}=-t \sum_{j=1}^{N-1}\left(c_{j+1}^{\dagger} c_{j}+\text { H.c. }\right)
$$

in the basis of Wannier states 89 This is a discrete version of the problem of the particle in the box [CTDL77, vol. 1 p. 77 et seq.], which is diagonal in the basis of plane waves $\sqrt{2 /(N+1)} \sin \left(k_{n} j\right)$ with $k_{n}=n \pi /(N+1), n \in\{1 \ldots N\}$ and dispersion $\varepsilon\left(k_{n}\right)=-2 t \cos \left(k_{n}\right)$, cf. fig. 2.1 (a). Thus, the energy band has finite support $[-B / 2, B / 2]$ with bandwidth $B=4 t$. Localized site and hopping impurities may be included by adding terms $V_{j} c_{j}^{\dagger} c_{j}$ respectively $-\left(t_{j, j+1}-t\right) c_{j+1}^{\dagger} c_{j}+$ H.c. at the site or bond of choice.

The two-particle part is given by

$$
H^{\mathrm{int}}=\sum_{j=1}^{N-1} U_{j, j+1}\left(n_{j}-\nu(n, U)\right)\left(n_{j+1}-\nu(n, U)\right)
$$

\footnotetext{
${ }^{6}$ Despite the fact that Tomonaga-Luttinger-liquid is the more appropriate nomenclature, in the following merely the term Luttinger liquid is used.

${ }^{7}$ One-particle Hamiltonians are labeled with lowercase letters, more-than-one-particle Hamiltonians with uppercase letters.

${ }^{8}$ The lattice constant $a$ of the chain is used as length scale (cf. appendix A), such that any location $x=j a=j$.

${ }^{9}$ Compare with Cal74, p. 291] or [Dat95, p. 141] for an introduction to the tight-binding method.
} 


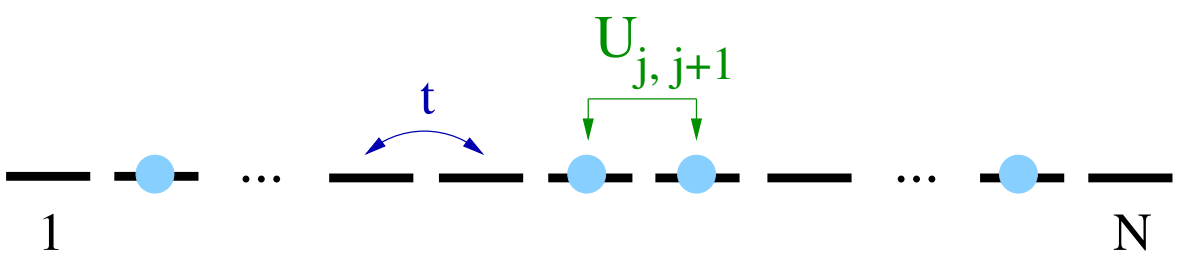

Figure 2.2: finite one-dimensional hopping chain with nearest-neighbour hopping $t$ and nearest-neighbour interaction $U$.

with the factor $\nu$ fixing the filling of the chain. Half-filling is obtained by setting $\nu=1 / 2$, e. g. [Sha94, p. 154]. Finally the complete Hamiltonian is denoted

$$
H^{\text {wire }}=h^{\text {wire }}+H^{\text {int }} \text {. }
$$

The clean system with homogeneous interaction $\left(U_{j, j+1}=U\right.$ for all $\left.j\right)$ can be obtained by a mapping from the XXZ-model (Jordan-Wigner transformation), cf. [JW28] and [NO98, p. 432], which is integrable and whose Bethe-ansat ${ }^{10}$ equations are known [YY66a, YY66b, YY66c]. Within a wide range of the filling $n$ and the interaction strength $U / t$, the low-energy properties of the model can be described by Luttinger-liquid theory [Hal80], cf. fig. 2.3. In the case of half-filling, this Luttinger-liquid behavior is restricted to $|U / t|<2$. The two Luttinger-liquid parameters $K$ and $v, 2.1$, can be obtained in a closed form ${ }^{11}$

$$
K^{-1}=\sqrt{\frac{v_{N}}{v_{J}}}=\frac{2}{\pi} \arccos \left(-\frac{U}{2 t}\right) \quad \text { and } \quad v=\sqrt{v_{N} v_{J}}=\frac{\pi t \sqrt{1-\left(\frac{U}{2 t}\right)^{2}}}{\pi-\arccos \left(-\frac{U}{2 t}\right)}
$$

cf. [Hal80, p. 1360] in combination with the identification $\Delta=-U /(2 t)$ of the original parameter $\Delta$ in the XXZ-model [YY66c, appendix A]. For $U / t>2$ a charge density wave builds up and for $U / t<-2$ phase separation occurs, cf. [Hal80] (or [Sch03, p. 119] for more intuitive arguments).

The leads, which the finite chain (2.5) will be coupled to, are modelled by semiinfinite hopping stripes at half-filling (i. e. with chemical potential being zero)

$$
h_{a}^{2 \mathrm{~d}-\text { lead }}=-\sum_{\vec{\jmath}, \vec{\jmath}^{\prime} \in \mathbb{A}_{a}}\left(t_{a}^{\left(\vec{\jmath}, \vec{\jmath}^{\prime}\right)} d_{\vec{\jmath}, a}^{\dagger} d_{\vec{\jmath}^{\prime}, a}+\text { H.c. }\right)
$$

with $\mathbb{A}_{a}=\left\{\left(j_{x}, j_{y}\right)^{\mathrm{T}} \mid j_{x} \in\left\{1, \ldots, N_{a}^{\text {con }}\right\} \wedge j_{y} \in\{1, \ldots, \infty\}\right\}$ and $t_{a}^{\left(\vec{\jmath} \vec{\jmath}^{\prime}\right)}$ connecting nearest-neighbour sites. The subscript $a$ identifies the left $(a=L)$ or the right $(a=R)$ lead. For later reference note that $\bar{a}$ defines the respective opposite of $a$, i. e. $a=L$ implies $\bar{a}=R$ and vice versa. By setting $N_{a}^{\text {con }}=1$ and dropping the redundant indices and vector arrows the one-dimensional lead (chain) is obtained

$$
h_{a}^{1 \mathrm{~d}-\text { lead }}=-t_{a} \sum_{j=1}^{\infty}\left(d_{j+1, a}^{\dagger} d_{j, a}+\text { H.c. }\right) \text {. }
$$

\footnotetext{
${ }^{10}$ Compare with Bet31 for the original idea or the triple KM97, KHM98, KHM00 for a review.

${ }^{11}$ Away from half-filling these parameters have to be obtained numerically, cf. e. g. Gia04, p. 146 et. seq.].
} 
Figure 2.3: ground-state phase diagram of the homogeneous chain with abbreviations: LL: Luttinger liquid, CDW: charge density wave, PS: phase separation; following [Hal80, fig. 1].

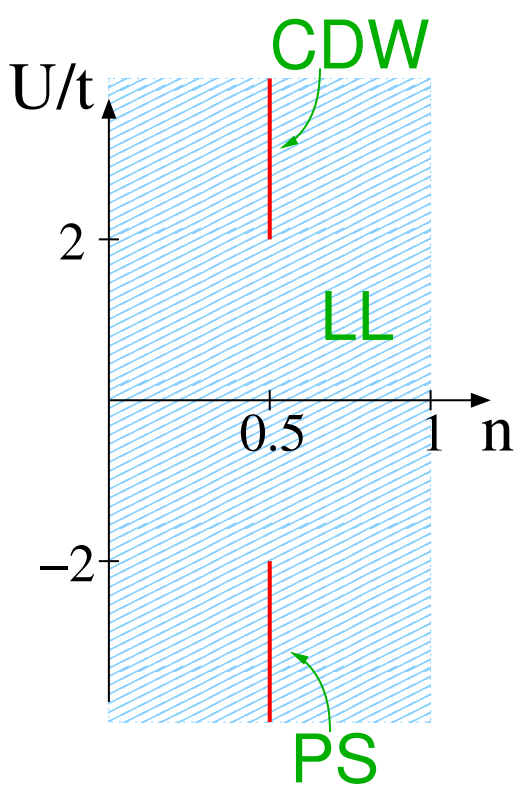

The properties of these models are discussed in detail in appendix D, The coupling geometry of the wire and the leads is defined by

$$
h_{a}^{\text {coupl }}=\sum_{j=1}^{N} \sum_{\vec{l} \in \mathbb{A}_{a}}\left(V_{a}^{(j, \vec{l})} c_{j}^{\dagger} d_{\vec{l}, a}+\text { H.c. }\right)
$$

with a coupling matrix $V_{a}^{(j, \vec{l})}$, which will be set up explicitly for the specific problems in chapter 4 and 5 . The one-dimensional case can be obtained as above. 


\section{computational methods}

\section{summary}

Primarily, in this chapter, the one-particle-irreducible scheme of the functional renormalization group is presented in detail and the approximation scheme, identified in the introduction as particularly useful method for studying Luttinger-liquid physics in fermionic lattice models is derived, cf. section 3.1. Important remarks on the accuracy of this approximate fRG scheme are made at the end of section 3.1.2. In section 3.2, the poor man's RG, which is made use of in chapter 4 to supplement the conductance calculations with the fRG is introduced and its derivation is sketched. The relation to the $\mathrm{fRG}$ is shortly commented on.

The computation of the observables studied in chapters 4 and 5 is explained in section 3.3. Particularly, in subsection 3.3.1, the calculation of the conductance for a wire contacted by two-dimensional leads within the framework of the LandauerBüttiker formula is derived by generalizing the one-dimensional case.

\section{the functional renormalization group}

Suppose $S$ being the action of a many-fermion system, explicitly

$$
\begin{aligned}
S= & \int d \xi^{\prime} d \xi \mathcal{G}_{0}^{-1}\left(\xi^{\prime}, \xi\right) \bar{\psi}\left(\xi^{\prime}\right) \psi(\xi)+\int d \xi^{\prime} d \xi V\left(\xi^{\prime}, \xi\right) \bar{\psi}\left(\xi^{\prime}\right) \psi(\xi) \\
& +\int d \xi_{1}^{\prime} d \xi_{2}^{\prime} d \xi_{1} d \xi_{2} I\left(\left\{\xi^{\prime} ; \xi\right\}\right) \bar{\psi}\left(\xi_{1}^{\prime}\right) \bar{\psi}\left(\xi_{2}^{\prime}\right) \psi\left(\xi_{1}\right) \psi\left(\xi_{2}\right)
\end{aligned}
$$

with Graßmann variables $\psi$ and a general set of quantum numbers and fermionic Matsubara-frequencies represented by $\xi$, cf. [NO98, p. 57 et seq.]. The free propagator $\mathcal{G}_{0}$ and $V$ contain the one-particle terms of the system and $I$ its antisymmetrized two-particle interaction terms. Furthermore, the first term in the equation defines the free action $S_{0}$, the other terms are subsummed in $S_{1}$, thus $S=S_{0}+S_{1}$.

The following standard definitions in terms of Graßmann-variables are used. The n-particle Green function reads

$$
\mathcal{G}\left(\xi_{1}^{\prime}, \xi_{2}^{\prime}, \ldots, \xi_{n}^{\prime} ; \xi_{1}, \xi_{2}, \ldots, \xi_{n}\right)=(-1)^{n}\left\langle\psi\left(\xi_{1}\right) \ldots \psi\left(\xi_{n}\right) \bar{\psi}\left(\xi_{n}^{\prime}\right), \ldots, \bar{\psi}\left(\xi_{1}^{\prime}\right)\right\rangle
$$


with the average $\langle\bullet\rangle:=Z^{-1} \int d \psi d \bar{\psi} \bullet \exp (-S)$, normalized by the partition function $Z:=\int d \psi d \bar{\psi} \exp (-S)$. Adding source fields $\eta(\xi)$ and $\bar{\eta}(\xi)$ to the action $S$ yields the generating functional $\mathscr{G}$ of the Green functions:

$$
\mathscr{G}(\{\eta\}\{\bar{\eta}\})=\left\langle\exp \left(-\int d \xi(\bar{\psi}(\xi) \eta(\xi)+\bar{\eta}(\xi) \psi(\xi))\right)\right\rangle .
$$

The logarithm of this functional, $\mathscr{W}(\{\eta\}\{\bar{\eta}\})=\ln (\mathscr{G}(\{\eta\}\{\bar{\eta}\}))$, singles out all disconnected Green functions, thus constituting the generating functional of the connected Green functions. Its Legendre transform

$$
\begin{aligned}
\Gamma(\{\phi\}\{\bar{\phi}\}) & -\int d \xi_{1} \int d \xi_{2} \bar{\phi}\left(\xi_{1}\right) \mathcal{G}_{0}^{-1}\left(\xi_{1}, \xi_{2}\right) \phi\left(\xi_{2}\right) \\
& =-\mathscr{W}(\{\eta\}\{\bar{\eta}\})-\int d \xi(\bar{\phi}(\xi) \eta(\xi)+\bar{\eta}(\xi) \phi(\xi))
\end{aligned}
$$

(called effective potential or effective action) with the new Graßmann fields

$$
\phi(\xi)=-\frac{\delta}{\delta \bar{\eta}(\xi)} \mathscr{W}(\{\eta\}\{\bar{\eta}\}) \text { and } \bar{\phi}(\xi)=\frac{\delta}{\delta \eta(\xi)} \mathscr{W}(\{\eta\}\{\bar{\eta}\})
$$

generates the one-particle-irreducible, connected, amputated n-particle Green functions $\gamma_{n}$ (vertex functions). These are the Green functions, which are connected such that they cannot be separated in disconnected parts by removing a single internal propagator (one-particle irreducibility) and whose external propagators are removed (amputated). The second term in the first line of $(3.2)$ is non-standard, it was added to later cancel out vacuum-energy terms in the flow equation for $\Gamma$, cf. [Wet01].

The vertex functions are the (functional) derivatives of the effective potential

$$
\gamma_{n}\left(\xi_{1}^{\prime}, \ldots, \xi_{n}^{\prime} ; \xi_{1}, \ldots, \xi_{n}\right)=\left.\frac{\delta^{2 n} \Gamma(\{\phi\}\{\bar{\phi}\})}{\delta \bar{\phi}\left(\xi_{1}^{\prime}\right) \cdots \delta \bar{\phi}\left(\xi_{n}^{\prime}\right) \delta \phi\left(\xi_{n}\right) \cdots \delta \phi\left(\xi_{1}\right)}\right|_{\phi=\bar{\phi}=0}
$$

\section{basics of the one-particle irreducible scheme}

The starting point of the fRG scheme is the introduction of a cutoff $\Lambda$ in the free propagator $\mathcal{G}_{0}\left(\xi^{\prime}, \xi\right)$ such that for $\Lambda=0$ the original system is recovered, i. e. $\mathcal{G}_{0}^{\Lambda=0}\left(\xi^{\prime}, \xi\right)=\mathcal{G}_{0}\left(\xi^{\prime}, \xi\right)$ and for $\Lambda=\infty$ all degrees of freedom are removed, i. e. $\mathcal{G}_{0}^{\Lambda=\infty}\left(\xi^{\prime}, \xi\right)=0$. This introduces a $\Lambda$-dependence in all Green functions and their generating functionals. Explicitly, the effective potential reads

$$
\begin{aligned}
\Gamma^{\Lambda}(\{\phi\}\{\bar{\phi}\}) & -\int d \xi_{1} \int d \xi_{2} \bar{\phi}\left(\xi_{1}\right)\left[\mathcal{G}_{0}^{\Lambda}\right]^{-1}\left(\xi_{1}, \xi_{2}\right) \phi\left(\xi_{2}\right) \\
& =-\mathscr{W}^{\Lambda}\left(\left\{\eta^{\Lambda}\right\}\left\{\bar{\eta}^{\Lambda}\right\}\right)-\int d \xi\left(\bar{\phi}(\xi) \eta^{\Lambda}(\xi)+\bar{\eta}^{\Lambda}(\xi) \phi(\xi)\right)
\end{aligned}
$$


whereat the $\Lambda$-dependence is introduced in the $\eta$ over their connection (3.3) to the $\phi$, which are the fundamental variables of $\Gamma$. The derivative of $\Gamma$ with respect to $\Lambda$ is given by $\mathrm{Med}$, $\mathrm{SH} 01$

$$
\begin{aligned}
\partial_{\Lambda} \Gamma^{\Lambda}(\{\phi\}\{\bar{\phi}\})=\int d \xi_{1} & \int d \xi_{2} \partial_{\Lambda}\left[\mathcal{G}_{0}^{\Lambda}\right]^{-1}\left(\xi_{1}, \xi_{2}\right) \mathcal{G}_{0}^{\Lambda}\left(\xi_{2}, \xi_{1}\right) \\
& -\int d \xi_{1} \int d \xi_{2} \partial_{\Lambda}\left[\mathcal{G}_{0}^{\Lambda}\right]^{-1}\left(\xi_{1}, \xi_{2}\right) \frac{\delta^{2} \mathscr{W}^{\Lambda}(\{\eta\}\{\bar{\eta}\})}{\delta \bar{\eta}^{\Lambda}\left(\xi_{2}\right) \delta \eta^{\Lambda}\left(\xi_{1}\right)} .
\end{aligned}
$$

Using the relation [NO98, p. 111 et seq.]

$$
\begin{aligned}
& \left(\begin{array}{cc}
\frac{\delta^{2} \mathscr{W}^{\Lambda}(\{\eta\}\{\bar{\eta}\})}{\delta \bar{\eta}^{\Lambda} \delta \eta^{\Lambda}} & -\frac{\delta^{2} \mathscr{W}^{\Lambda}(\{\eta\}\{\bar{\eta}\})}{\delta \bar{\eta}^{\Lambda} \delta \bar{\eta}^{\Lambda}} \\
-\frac{\left.\delta^{2} \mathscr{W}^{\Lambda}(\{\eta\} \bar{\eta}\}\right)}{\delta \eta^{\Lambda} \delta \eta^{\Lambda}} & \frac{\delta^{2} \mathscr{W}^{\Lambda}(\{\eta\}\{\bar{\eta}\})}{\delta \eta^{\Lambda} \delta \bar{\eta}^{\Lambda}}
\end{array}\right) \\
= & \left(\begin{array}{cc}
\frac{\delta^{2} \Gamma^{\Lambda}(\{\phi\}\{\bar{\phi}\})}{\delta \bar{\phi} \delta \phi}+\left[\mathcal{G}_{0}^{\Lambda}\right]^{-1} & \frac{\delta^{2} \Gamma^{\Lambda}(\{\phi\}\{\bar{\phi}\})}{\delta \bar{\delta} \delta \bar{\phi}} \\
\frac{\delta^{2} \Gamma^{\Lambda}(\{\phi\}\{\bar{\phi}\})}{\delta \phi \delta \phi} & \frac{\delta^{2} \Gamma^{\Lambda}(\{\phi\}\{\bar{\phi}\})}{\delta \phi \delta \bar{\phi}}-\left(\left[\mathcal{G}_{0}^{\Lambda}\right]^{-1}\right)^{T}
\end{array}\right)^{-1} \\
= & \left(\begin{array}{ll}
A_{1} & A_{2} \\
A_{3} & A_{4}
\end{array}\right)
\end{aligned}
$$

the flow equation for the effective potential is obtained as

$$
\begin{aligned}
\partial_{\Lambda} \Gamma^{\Lambda}(\{\phi\}\{\bar{\phi}\})= & \int d \xi_{1} \int d \xi_{2} \partial_{\Lambda}\left[\mathcal{G}_{0}^{\Lambda}\right]^{-1}\left(\xi_{1}, \xi_{2}\right) \mathcal{G}_{0}^{\Lambda}\left(\xi_{2}, \xi_{1}\right) \\
& -\int d \xi_{1} \int d \xi_{2} \partial_{\Lambda}\left[\mathcal{G}_{0}^{\Lambda}\right]^{-1}\left(\xi_{1}, \xi_{2}\right) A_{1}\left(\xi_{2}, \xi_{1}\right)
\end{aligned}
$$

with initial value [Med, p. 19]

$$
\Gamma^{\Lambda=\infty}(\{\phi\}\{\bar{\phi}\})=S_{1}(\{\phi\}\{\bar{\phi}\}) .
$$

Equations (3.6) and (3.7) constitute an initial value problem for the generating functional $\Gamma$ of the vertex functions $\gamma_{n}$. By expanding $\Gamma$ in a Volterra series, an infinite system of coupled differential equations can be deduced for the $\gamma_{n}$. Explicitely, the expansion reads

$$
\begin{aligned}
\Gamma^{\Lambda}(\{\phi\}\{\bar{\phi}\})=\sum_{n=0}^{\infty} \frac{(-1)^{n}}{n !^{2}} \int \prod_{j=1}^{n}\left(d \xi_{j} d \xi_{j}^{\prime}\right) \gamma_{n}\left(\xi_{1}^{\prime}, \ldots,\right. & \left.\xi_{n}^{\prime} ; \xi_{1}, \ldots, \xi_{n}\right) \\
& \times \bar{\phi}\left(\xi_{1}^{\prime}\right) \cdots \bar{\phi}\left(\xi_{n}^{\prime}\right) \phi\left(\xi_{n}\right) \cdots \phi\left(\xi_{1}\right)
\end{aligned}
$$

with the definition (3.4) inserted as expansion coefficients. Upon inserting this series on the left- and right-hand side of (3.6) and arranging the resulting polynomial in powers of $\phi$ and $\bar{\phi}$, the differential equations for the $\gamma_{n}$ are obtained by comparing 
the respective coefficients [Med, p. 20 et seq.], [SH01, p. 14 et seq.]. The first three equations are given as

$$
\begin{aligned}
& \partial_{\Lambda} \gamma_{0}^{\Lambda}= \int d \xi_{1} d \xi_{1}^{\prime}\left[\mathcal{G}^{\Lambda}-\mathcal{G}_{0}^{\Lambda}\right]\left(\xi_{1}, \xi_{1}^{\prime}\right) \partial_{\Lambda}\left[\mathcal{G}_{0}^{\Lambda}\right]^{-1}\left(\xi_{1}^{\prime}, \xi_{1}\right) \\
& \partial_{\Lambda} \gamma_{1}^{\Lambda}\left(\xi_{1}^{\prime}, \xi_{1}\right)=\int d \xi_{2} d \xi_{2}^{\prime} \mathcal{S}^{\Lambda}\left(\xi_{2}, \xi_{2}^{\prime}\right) \gamma_{2}^{\Lambda}\left(\xi_{1}^{\prime}, \xi_{2}^{\prime} ; \xi_{1}, \xi_{2}\right)(3.8 \mathrm{a}) \\
& \partial_{\Lambda} \gamma_{2}^{\Lambda}\left(\xi_{1}^{\prime}, \xi_{2}^{\prime} ; \xi_{1}, \xi_{2}\right)=-\int d \xi_{3} d \xi_{3}^{\prime} \gamma_{3}^{\Lambda}\left(\xi_{1}^{\prime}, \xi_{2}^{\prime}, \xi_{3}^{\prime} ; \xi_{1}, \xi_{2}, \xi_{3}\right) \mathcal{S}^{\Lambda}\left(\xi_{3}, \xi_{3}^{\prime}\right) \\
&+\int d \xi_{3} d \xi_{3}^{\prime} d \xi_{4} d \xi_{4}^{\prime} \mathcal{G}^{\Lambda}\left(\xi_{3}, \xi_{3}^{\prime}\right) \mathcal{S}^{\Lambda}\left(\xi_{4}, \xi_{4}^{\prime}\right) \\
& \times\left\{\gamma_{2}^{\Lambda}\left(\xi_{1}^{\prime}, \xi_{2}^{\prime} ; \xi_{3}, \xi_{4}\right) \gamma_{2}^{\Lambda}\left(\xi_{3}^{\prime}, \xi_{4}^{\prime} ; \xi_{1}, \xi_{2}\right)\right. \\
&-\gamma_{2}^{\Lambda}\left(\xi_{1}^{\prime}, \xi_{4}^{\prime} ; \xi_{1}, \xi_{3}\right) \gamma_{2}^{\Lambda}\left(\xi_{3}^{\prime}, \xi_{2}^{\prime} ; \xi_{4}, \xi_{2}\right)-\left[3 \leftrightarrow 4 ; 3^{\prime} \leftrightarrow 4^{\prime}\right] \\
&\left.+\gamma_{2}^{\Lambda}\left(\xi_{2}^{\prime}, \xi_{4}^{\prime} ; \xi_{1}, \xi_{3}\right) \gamma_{2}^{\Lambda}\left(\xi_{3}^{\prime}, \xi_{1}^{\prime} ; \xi_{4}, \xi_{2}\right)+\left[3 \leftrightarrow 4 ; 3^{\prime} \leftrightarrow 4^{\prime}\right]\right\}
\end{aligned}
$$

with $\mathcal{S}^{\Lambda}:=\mathcal{G}^{\Lambda} \partial_{\Lambda}\left[\mathcal{G}_{0}^{\Lambda}\right]^{-1} \mathcal{G}^{\Lambda}$ (single-scale propagator). Note that this system is not closed as $\gamma_{3}^{\Lambda}$ enters $\gamma_{2}^{\Lambda}, \gamma_{4}^{\Lambda}$ enters $\gamma_{3}^{\Lambda}$ and so on. The initial values $\gamma_{n}^{\Lambda=\infty}$ follow from the initial value of $\Gamma$ as the (antisymmetrized) interactions of the system:

$$
\begin{aligned}
\gamma_{0}^{\Lambda=\infty} & =0 \\
\gamma_{1}^{\Lambda=\infty}\left(\xi_{1}^{\prime}, \xi_{1}\right) & =-V\left(\xi_{1}^{\prime}, \xi_{1}\right) \\
\gamma_{2}^{\Lambda=\infty}\left(\xi_{1}^{\prime}, \xi_{2}^{\prime} ; \xi_{1}, \xi_{2}\right) & =I\left(\xi_{1}^{\prime}, \xi_{2}^{\prime} ; \xi_{1}, \xi_{2}\right) .
\end{aligned}
$$

For $n \geq 3$ all $\gamma_{n}^{\Lambda=\infty}=0$, because no $(n>2)$-particle interactions were included in the definition of $S$, cf. (3.1). To put some physical intuition to these equations, note that the vertex functions with $n \geq 2$ represent the coupling strength of the respective $n$-particle interaction. The function $\gamma_{1}$ can be identified as the system's self-energy $\Sigma$, explicitly $\gamma_{1}\left(\xi^{\prime}, \xi\right)=-\Sigma\left(\xi^{\prime}, \xi\right)$ [Med, p. 10]. Finally a slight re-definition of the average $\langle\bullet\rangle$ allows for the identification of $\gamma_{0}$ as the grand-canonical potential of the system, cf. [WMS07b] and [AEM+04] for details and applications.

\section{adaption for one-dimensional lattice systems}

In this subsection, the general fRG scheme is operationalized for a one-dimensional hopping chain filled with spinless fermions, which interact via nearest-neighbour interactions, as introduced in section 2.2, eq. (2.5). The approximation scheme was developed in $\left.\mathrm{AEM}^{+} 04\right]$ and $\left[\mathrm{EMA}^{+}\right.$05], extending the scheme of [MMSS02b] and MMSS02a by including an approximate flow of the two-particle interaction $\gamma_{2}^{\Lambda}$ without any noticeable change in the computational effort. This scheme will be referred to as approximate $f R G$ or approximate $f R G$ scheme in the following. 
First the infinite system is truncated by neglecting all $(n>2)$-particle interactions, namely by neglecting the term containing $\gamma_{3}^{\Lambda}$ in equation (3.8c). The initial value of $\gamma_{3}$ is zero, however, it is generated during the flow by terms of third order in $\gamma_{2}^{\Lambda}$, cf. [AEM $\left.{ }^{+} 04\right]$ and [SH01, §5]. Thus, as long as $\gamma_{2}^{\Lambda}$ stays sufficiently small, its contribution may safely be discarded. Thereby a closed system for the self-energy $\Sigma^{\Lambda}$ and the two-particle interaction $\gamma_{2}^{\Lambda}$ is obtained.

Next, the external Matsubara frequencies in $\gamma_{2}^{\Lambda}\left(\xi_{1}^{\prime}, \xi_{2}^{\prime} ; \xi_{1}, \xi_{2}\right)$ are set to zero. Thus, the frequency independence of the initial interaction is preserved during the flow. As a consequence also the self-energy looses its dependence on the Matsubara frequencies. This simplification introduces errors of second respectively third order in the interaction strength in $\Sigma^{\Lambda}$ and $\gamma_{2}^{\Lambda}\left[\mathrm{AEM}^{+} 04\right]$. On the other hand, the computational effort is decisively reduced, cf. the attempts to include the frequency

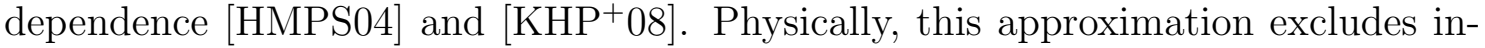
elastic processes and limits the accuracy to which scaling exponents can be obtained, cf. next subsection.

At this point the cutoff procedure is made explicit by introducing a step function in the Matsubara frequencies into the bare propagator [AEM ${ }^{+} 04$

$$
\mathcal{G}_{0}^{\Lambda}=\Theta\left(\left|\omega_{n}\right|-\Lambda\right) \mathcal{G}_{0}
$$

and expanding $\int d \xi \rightarrow \int d \alpha \sum_{\omega_{n}}$ with some set of quantum numbers $\alpha$. Following the reasoning of $\left[\mathrm{AEM}^{+} 06 \mathrm{~b}\right.$, appendix $\left.\mathrm{A}\right]$ to express the sum over $\omega_{n}$ as an integral in $\omega$ over a function which is piecewise constant on intervals centered around the Matsubara frequencies $\omega_{n}$, Morris lemma [Mor11, p. 2422] can be applied just as in the case of $T=0\left[\mathrm{AEM}^{+} 04\right]$. This procedure clearly outperforms older versions using a smooth cutoff, cf. [EMA $\left.{ }^{+} 05\right]$ and [Ens05, p. 64 et seq.], concerning the speed of the calculations. The frequency integrated differential equations for $\Sigma^{\Lambda}$ and $\gamma_{2}^{\Lambda}$ obtained this way are given as

$$
\begin{array}{r}
\partial_{\Lambda} \Sigma^{\Lambda}\left(\alpha_{1}^{\prime}, \alpha_{1}\right)=\frac{-1}{2 \pi} \int d \alpha_{2} \int d \alpha_{2}^{\prime} \sum_{\omega= \pm \hat{\Lambda}} \tilde{\mathcal{G}}^{\Lambda}\left(\alpha_{2}, \alpha_{2}^{\prime}, \imath \omega\right) \gamma_{2}^{\Lambda}\left(\alpha_{1}^{\prime}, \alpha_{2}^{\prime} ; \alpha_{1}, \alpha_{2}\right) \\
\partial_{\Lambda} \gamma_{2}^{\Lambda}\left(\alpha_{1}^{\prime}, \alpha_{2}^{\prime} ; \alpha_{1}, \alpha_{2}\right)=\frac{1}{2 \pi} \int d \alpha_{3} d \alpha_{3}^{\prime} d \alpha_{4} d \alpha_{4}^{\prime} \sum_{\omega= \pm \hat{\Lambda}}\left\{\frac{1}{2} \tilde{\mathcal{G}}^{\Lambda}\left(\alpha_{3}, \alpha_{3}^{\prime}, \imath \omega\right)\right. \\
\times \tilde{\mathcal{G}}^{\Lambda}\left(\alpha_{4}, \alpha_{4}^{\prime},-\imath \omega\right) \gamma_{2}^{\Lambda}\left(\alpha_{1}^{\prime}, \alpha_{2}^{\prime} ; \alpha_{3}, \alpha_{4}\right) \gamma_{2}^{\Lambda}\left(\alpha_{3}^{\prime}, \alpha_{4}^{\prime} ; \alpha_{1}, \alpha_{2}\right) \\
+\tilde{\mathcal{G}}^{\Lambda}\left(\alpha_{3}, \alpha_{3}^{\prime}, \imath \omega\right) \tilde{\mathcal{G}}^{\Lambda}\left(\alpha_{4}, \alpha_{4}^{\prime}, \imath \omega\right) \\
{\left[-\gamma_{2}^{\Lambda}\left(\alpha_{1}^{\prime}, \alpha_{4}^{\prime} ; \alpha_{1}, \alpha_{3}\right) \gamma_{2}^{\Lambda}\left(\alpha_{3}^{\prime}, \alpha_{2}^{\prime} ; \alpha_{4}, \alpha_{2}\right)\right.} \\
\left.\left.+\gamma_{2}^{\Lambda}\left(\alpha_{2}^{\prime}, \alpha_{4}^{\prime} ; \alpha_{1}, \alpha_{3}\right) \gamma_{2}^{\Lambda}\left(\alpha_{3}^{\prime}, \alpha_{1}^{\prime} ; \alpha_{4}, \alpha_{2}\right)\right]\right\}
\end{array}
$$


with $\tilde{\mathcal{G}}^{\Lambda}:=\left(\mathcal{G}_{0}^{-1}-\Sigma^{\Lambda}\right)^{-1}$ and $\hat{\Lambda}$ representing the Matsubara frequency closest to $\Lambda$. The corresponding initial values $(3.9)$ read

$$
\begin{aligned}
\Sigma^{\hat{\Lambda}_{0}}\left(\alpha_{1}^{\prime}, \alpha_{1}\right) & =V\left(\alpha_{1}^{\prime}, \alpha_{1}\right)+\frac{1}{2} \sum_{\alpha_{2}} I\left(\alpha_{1}^{\prime}, \alpha_{2} ; \alpha_{1}, \alpha_{2}\right) \\
\gamma_{2}^{\hat{\Lambda}_{0}}\left(\alpha_{1}^{\prime}, \alpha_{2}^{\prime} ; \alpha_{1}, \alpha_{2}\right) & =I\left(\alpha_{1}^{\prime}, \alpha_{2}^{\prime} ; \alpha_{1}, \alpha_{2}\right),
\end{aligned}
$$

whereat $\hat{\Lambda}_{0}$ is some huge, yet finite and thus numerically accessible, number (typically $\left.\approx 10^{8}\right)$. The second term in the initial value of $\Sigma^{\Lambda}$ accounts for $\hat{\Lambda}_{0}<\infty$, cf. $\mathrm{AEM}^{+} 04$ and $[\mathrm{Wäc06}, \mathrm{p}$. xvii] for details.

On the right-hand side of the flow equation (3.10b) the full Green function $\tilde{\mathcal{G}}$ appears. This feeds back the influence of the one-particle potential $V\left(\alpha_{1}^{\prime}, \alpha_{1}\right)$ into the two-particle interaction and becomes important for systems containing a high number of randomly distributed inhomogeneities (disordered systems) Gia04, p. 285 et seq.]. However, for the treatment of isolated structures in otherwise clean systems as considered in this thesis, this feedback can be neglected safely [Gia04, p. 323]. We therefore replace $\tilde{\mathcal{G}} \rightarrow \mathcal{G}_{0}$ in $(3.10 \mathrm{~b})$.

For a one-dimensional lattice with $N$ sites, the number of flow equations for $\gamma_{2}^{\Lambda}\left(\alpha_{1}^{\prime}, \alpha_{2}^{\prime}, \alpha_{1}, \alpha_{2}\right)$ is proportional to $N^{4}$ (if not reduced by symmetry), which has to be reduced significantly by a suitable parametrization of $\gamma_{2}^{\Lambda}$. As the focus of this thesis lies on asymptotic low-energy behavior, the interaction $\gamma_{2}^{\Lambda}$ may be projected onto the Fermi points, which by antisymmetry gives the single term $\gamma_{2}^{\Lambda}\left(k_{F},-k_{F} ; k_{F},-k_{F}\right)$. Furthermore, the interaction is pinned to the nearest-neighbour structure of the bare interaction (2.4) in position space $\left(\alpha_{i}=j_{i}\right)$, viz.

$$
\gamma_{2}^{\Lambda}\left(j_{1}^{\prime}, j_{2}^{\prime} ; j_{1}, j_{2}\right)=U^{\Lambda}\left(\delta_{j_{1} j_{2}-1}+\delta_{j_{1} j_{2}+1}\right)\left(\delta_{j_{1} j_{1}^{\prime}} \delta_{j_{2} j_{2}^{\prime}}-\delta_{j_{1} j_{2}^{\prime}} \delta_{j_{2} j_{1}^{\prime}}\right),
$$

which in $k$-space reads $\gamma_{2}^{\Lambda}\left(k_{F},-k_{F} ; k_{F},-k_{F}\right)=2 U^{\Lambda}\left(1-\cos \left(2 k_{F}\right)\right)$. Upon inserting this expression into $3.10 \mathrm{~b}$, the flow equation can be integrated analytically, yielding at half filling

$$
U^{\Lambda}=\frac{U}{1+\left(\Lambda-\frac{2+\Lambda^{2}}{\sqrt{4+\Lambda^{2}}}\right) \frac{U}{2 \pi}},
$$

cf. [AEM ${ }^{+}$04] and for details [And06, p. 39 and p. 140 et seq.]. In order to treat inhomogeneous interactions, cf. (2.4), this equation is applied locally on each bond, giving

$$
U_{j, j+1}^{\Lambda}=\frac{U_{j, j+1}}{1+\left(\Lambda-\frac{2+\Lambda^{2}}{\sqrt{4+\Lambda^{2}}}\right) \frac{U_{j, j+1}}{2 \pi}},
$$

which constitutes a minor additional approximation $\left[\mathrm{EMA}^{+} 05\right.$, p. 8].

Inserting the parametrization (3.12) into (3.10a), the flow equations of the selfenergy in position space $\left(\alpha_{i}=j_{i}\right)$ simplify to

$$
\begin{aligned}
\partial_{\Lambda} \Sigma^{\Lambda}(j, j) & =-\frac{1}{\pi} \sum_{r= \pm 1} U_{j, j+r}^{\Lambda} \mathfrak{R e} \tilde{\mathcal{G}}^{\Lambda}(j, j+r, \imath \hat{\Lambda}) \\
\partial_{\Lambda} \Sigma^{\Lambda}(j, j \pm 1) & =\frac{U_{j, j \pm 1}^{\Lambda}}{\pi} \mathfrak{R e} \tilde{\mathcal{G}}^{\Lambda}(j, j \pm 1, \imath \hat{\Lambda}) .
\end{aligned}
$$


The self-energy in position space becomes real and tridiagonal. Thus, at any stage of the flow $\Lambda: \infty \rightarrow 0$ the fRG provides a one-particle Hamiltonian $h^{\Lambda}=h^{\text {wire }}+\Sigma^{\Lambda}$ of similar form as the one-particle part of the original Hamiltonian (2.5), yet with on-site energies and hoppings renormalised by $\Sigma^{\Lambda}$. In particular, this is true for the end of the flow, which means that the many-particle problem at hand has eventually been mapped onto a one-particle Hamiltonian

$$
\bar{h}=h^{\text {wire }}+\Sigma,
$$

in which the effect of the two-particle interaction is taken into account by a potential term $\Sigma=\Sigma^{\Lambda=0}$ consisting of a local $\left(\Sigma_{j, j}\right)$ and a non-local $\left(\Sigma_{j, j+1}\right)$ part. This effective (scattering) potential renormalizes the (local) on-site energies as well as the (non-local) hopping amplitudes in the one-particle part of the original Hamiltonian.

Furthermore, for the calculation of $\Sigma^{\Lambda}$, only the sub-, superdiagonal and diagonal elements of the Green function $\tilde{\mathcal{G}}$ are needed. This result is important as it allows for the usage of highly efficient algorithms to set up the right-hand side of the flow equations for the self-energy (cf. appendix C), thereby allowing to treat system sizes, which permit to access the asymptotic low-energy behavior.

\section{comment on the degree of accuracy of the approximate fRG scheme}

A particularly important manifestation of Luttinger-liquid physics is the powerlaw behavior of a variety of observables as function of external parameters with exponents depending at fixed filling only on the Luttinger-liquid parameter $K$, cf. section 2.1. For small interactions (small $U$ ), the $U$-expansion of $K \operatorname{reads} K=$ $1-\frac{U}{U_{c}}+\mathcal{O}\left(\frac{U}{U_{c}}\right)^{2}$ with $U_{c}$ being a model and parameter dependent scale $\left(U_{c}=\pi t\right.$ in the case of $K$ given by (2.6)). Thus, the boundary exponent $\alpha_{\text {end }}=\frac{1}{K}-1 \approx \mathcal{O}\left(\frac{U}{U_{c}}\right)$ is of linear order in $U / U_{c}$, while the bulk exponent $\alpha_{\text {bulk }}=\left(K+\frac{1}{K}-2\right) / 2 \approx \mathcal{O}\left(\frac{U}{U_{c}}\right)^{2}$ is of quadratic order in $U / U_{c}$. The approximate $\mathrm{fRG}$ scheme presented above was shown to reproduce the scaling exponents known from alternative approaches (e. g. effective field theories) to linear order $\left[\mathrm{AEM}^{+} 04, \mathrm{EMA}^{+}\right.$05, BTSMS05b, which implies, that scaling with $\alpha_{\text {end }}$ can be calculated within this scheme, while scaling with $\alpha_{\text {bulk }}$ and thus bulk Luttinger-liquid behavior can not. If bulk Luttinger-liquid physics is dominant, the scaling exponent is therefore expected to vanish within the approximate fRG. This can be traced back to the fact, that terms of order $U^{2}$ are only partly kept in the flow of the self-energy, cf. the preceding section.

\section{projection of the one- and two-dimensional leads}

The leads (2.7) respectively (2.8), which are attached to the Hamiltonian (2.5) of the wire, can be integrated out at each step of the fRG-flow. Using Feshbach projection 
(cf. appendix B) with the projection operator $P$ onto the subspace of $h^{\text {wire }}$ and $Q:=1-P$, the Green function reads

$$
\begin{aligned}
P \tilde{\mathcal{G}}^{\Lambda}(\imath \hat{\Lambda}) P & =\left(\imath \hat{\Lambda} P-P h^{\Lambda} P-P h^{\Lambda} Q\left(\imath \hat{\Lambda} Q-Q h^{\Lambda} Q\right)^{-1} Q h^{\Lambda} P\right)^{-1} \\
& =\left(\imath \hat{\Lambda} P-h^{\text {wire }}-\Sigma^{\Lambda}-\left(h_{L}^{\text {coupl }}\right)^{\dagger} \mathcal{G}_{L}^{\text {lead }} h_{L}^{\text {coupl }}-\left(h_{R}^{\text {coupl }}\right)^{\dagger} \mathcal{G}_{\mathrm{R}}^{\text {lead }} h_{R}^{\text {coupl }}\right)^{-1}
\end{aligned}
$$

Thereby the problem is reduced to the inversion of an $(N \times N)$-matrix and the calculation of the Green function of the isolated leads $\mathcal{G}_{L}^{\text {lead }}$ and $\mathcal{G}_{R}^{\text {lead }}$. In chapter 4 and 5 this general procedure is applied and further specified.

\section{\begin{tabular}{l|l} 
poor man's RG & 3.2
\end{tabular}}

Consider a one-dimensional wire of interacting electrons with some (symmetric) one-particle potential at the origin, which, for non-interacting electrons, is specified by the transmission and reflection amplitudes $\left\{t_{0}, r_{0}\right\}$. In lowest-order perturbation theory the two-particle interaction constitutes additional scattering potentials (Hartree- and Fock-potential [AM76, p. 332 et seq.]), which are treated in the Born approximation [Sit71, p. 43 et seq.]. This leads to corrections $\Delta t_{k}$ and $\Delta r_{k}$ (k being the wave number of the scattering state), which diverge logarithmically at the Fermi points for all orders of perturbation theory in the interaction strength [MYG93].

This divergence originates from the slowly decaying Friedel oscillations Mah00, p. 195], which enter the Hartree potential over the electron density. Therefor Yue et al. set up the following RG scheme [MYG93, YYGM94] in the spirit of Anderson's "poor man's RG" And70]. A sufficiently small region $(-l, l)$ around the one-particle scattering potential at the origin is chosen and the perturbative correction to the bare amplitudes $\{t, r\}$ is calculated with the Hartree- and Fock-potential being cut off outside $(-l, l)$. This regularizes the divergence as the slow decay of the oscillations is cut off and yields some renormalized amplitudes $\left\{t+\Delta t_{k}, r+\Delta r_{k}\right\}$. Next, the region is scaled up by a factor $\Lambda \gg 1$, viz. $(-\Lambda l, \Lambda l)$, and the calculation is repeated with the renormalized amplitudes $\left\{t+\Delta t_{k}, r+\Delta r_{k}\right\}$ and the Hartree- and Fockpotential having support on $(-\Lambda l, \Lambda l)$. This procedure is iterated until a length scale is reached at which the phase coherence of the scattered electron and the Friedel oscillations is lost [YGM94]. Effectively, at the end of this RG scheme, the logarithmically divergent terms in the perturbation series of the corrections to the amplitudes are summed to infinite order in the two-particle interaction strength YGM94.

Due to the unitarity of the scattering matrix [Sit71, p. 11], only a single independent flow equation for the transmission amplitude is obtained, which for continuous $\Lambda$ reads [YGM94]

$$
\frac{d t_{k}^{\Lambda}}{d \ln \Lambda}=-\alpha t_{k}^{\Lambda}\left(1-\left|t_{k}^{\Lambda}\right|^{2}\right)
$$


with $\alpha=\left(1-K^{2}\right) /\left(1+K^{2}\right),(K$ : Luttinger-liquid parameter $)$. The parameter $\Lambda$ runs from 1 to $1 /\left(\left|k-k_{F}\right| d\right)$ with $d$ being the characteristic length scale of the twoparticle interaction and $k_{F}$ the Fermi wave number. The initial condition is $t_{k}^{\Lambda=1}=t$. This approach is valid for any strength of the bare one-particle potential. However, it is restricted to weak two-particle interactions due to their perturbative treatment [YGM94. Therefor, the Luttinger-liquid exponents are only correct to leading order in the interaction and thus bulk Luttinger-liquid behavior is not being captured. A detailed comparison of this approach to the previously presented approximate fRG scheme is made in [MAE ${ }^{+} 08$ ], suggesting the overall supremacy of the latter.

The poor man's RG scheme was generalized to a junction of $N$ one-dimensional wires, characterized by a scattering matrix $S$ with diagonal elements $r_{i i}$ (reflection amplitude from wire $i$ into wire $i$ ) and off-diagonal elements $t_{i j}$ (transmission amplitudes from wire $i$ into wire $j$ ) [LRS02]. Following above's procedure of Yue et al. an RG equation for $S$ is obtained:

$$
\frac{d S^{\Lambda}}{d \ln \Lambda}=S^{\Lambda}\left(F^{\Lambda}\right)^{\dagger} S^{\Lambda}-F^{\Lambda}
$$

with a diagonal matrix $F$ with elements $F_{j j}=-1 / 2 \alpha_{j} r_{j j}$ and $\alpha_{j}$ parametrizing the interaction strength in the $j$ th wire, cf. above. The flow again starts at $\Lambda=1$ and is stopped at a length scale at which the phase coherence of the scattered electron and the Friedel oscillations is lost.

Thus far, the RG-schemes have been set up for continuous wires. Connection to a microscopic lattice model of $N=3$ wires is obtained using the identities

$$
r_{i i}=\frac{2 u_{i}^{2}}{\sum_{l=1}^{3} u_{l}^{2}}-1 \quad \text { and } \quad t_{i j}=\frac{2 u_{i} u_{j}}{\sum_{l=1}^{3} u_{l}^{2}}
$$

with $-u_{i}$ being the hopping constant from the last site of the $i$ th wire to the connecting site [LRS02].

\section{computing observables}

In this subsection the observables which are examined in subsequent chapters are introduced and their calculation within the fRG and the poor man's RG is explained.

\section{conductance}

In linear response, the conductance $G$ of a system is defined as the proportionality constant relating an infinitesimal change $d V$ of the bias voltage $V$ to an infinitesimal change $d I$ of the current $I$, starting in the equilibrium situation $V=0$ [BF04, p. 97], i. e.

$$
G:=\left.\frac{d I}{d V}\right|_{V=0}
$$


Within the general setup considered in this work, i. e. a scattering region connected to two non-interacting reservoirs, this expression can be elaborated on within the Kubo formalism of linear response, eventually connecting $G$ to the retarded currentcurrent correlation function $C_{a \bar{a}}[\mathrm{BF} 04$, p. 99]:1]

$$
G=\lim _{\omega \rightarrow 0} \frac{C_{a \bar{a}}(\omega+\imath \eta)-C_{a \bar{a}}(0)}{\imath \omega}
$$

with $\eta \searrow 0$ and

$$
C_{a \bar{a}}\left(\imath \omega_{n}\right)=\int_{0}^{\beta} d \tau\left\langle\mathrm{T}_{\tau} J_{a}(\tau) J_{\bar{a}}(0)\right\rangle e^{\imath \omega_{n} \tau}
$$

The symbol $J_{a}$ denotes the particle-current operator, describing current from the left respectively right reservoir into the scattering region, cf. e. g. [CTDL77, vol. 1 p. 238].

For the particular case of a mesoscopic scattering region of non-interacting electrons, the conductance is completely determined by the probability with which an incident electron penetrates through this region, put explicitly:

$$
G / G_{0}=\int_{-\infty}^{\infty} d \varepsilon\left(-\partial_{\varepsilon} f\right) \mathcal{T}(\varepsilon)
$$

with the Fermi-function $f$ and the transmission probability $\mathcal{T}(\varepsilon)$ for an incoming electron. This point of view originates in the work of Landauer and Büttiker, cf. [Lan70, BILP85, Lan96, IL99]. Full derivations can e. g. be found in [CJM05] and [BF04, p. 112 et seq.].

For an interacting scattering region, corrections stemming from the two-particle interaction enter the basic formula (3.19), such that $\mathcal{T}(\varepsilon)$ becomes the sum of a contribution $\mathcal{T}^{(i)}(\varepsilon)$, which only involves bare current operators, and a contribution $\mathcal{T}^{(i i)}(\varepsilon)$ involving the vertex function $\gamma_{2}$. Within the approximate fRG scheme from section 3.1.2, $\mathcal{T}^{(i i)}(\varepsilon)$ can be shown to vanish. This originates in the frequencyindependence of $\gamma_{2}$. A detailed proof of this statement is given in appendix E.

The remaining transmission probability $\mathcal{T}^{(i)}$ can be obtained within the framework of one-particle scattering theory, using the effective Hamiltonian $\bar{h}$ obtained from the fRG flow. The explicit expressions and a detailed derivation are given in appendix E.

Within the poor man's approach, cf. section 3.2, the conductance is obtained from the $S$ matrix at the end of its renormalization flow, cf. section 4.1 .

\section{local spectral function}

From the one-particle Green function $\mathcal{G}$, the local spectral function can be extracted as [BF04, p. 128 et seq.] [Eco79, p. 10]

$$
\rho_{j}(\omega)=-\frac{1}{\pi} \mathfrak{I} \mathfrak{m} \mathcal{G}(j, j, \omega+\imath \eta)
$$

\footnotetext{
${ }^{1}$ Note that the Hamiltonians considered in this work are time-inversion symmetric, which implies that the choices $C_{L R}$ or $C_{R L}$ are equivalent leading to a $G$ being independent of this particular choice.
} 
with an infinitesimal $\eta \searrow 0$. Since the fRG procedure yields an effective oneparticle Hamiltonian $\bar{h}$, its resolvent $\mathcal{G}=(\omega+\imath \eta-\bar{h})^{-1}$ can directly be inserted into the equation. Note that the necessary inversion can be done efficiently with the algorithms presented in appendix C. The local spectral function is particularly useful in the context of the identification of Luttinger-liquid behavior due to its asymptotic suppression at the Fermi energy, cf. section 2.1.

\section{charging}

The average occupation (charging) $n_{j}$ of a given site $j$ of some lattice is by definition given as

$$
n_{j}:=\left\langle c_{j}^{\dagger} c_{j}\right\rangle=\frac{1}{\beta} \sum_{\omega_{n}} e^{-\imath \eta} \mathcal{G}\left(j, j, \imath \omega_{n}\right)
$$

with $\eta \nearrow 0$. The sum can be casted into an integral representation using Poisson's summation formula [BF04, p. 197], yielding

$$
n_{j}=\int_{\omega_{\min }}^{\omega_{\max }} d \omega f(\omega) \rho_{j}(\omega)
$$

The symbol $f$ denotes the Fermi function, $\rho_{j}$ is defined in equation (3.20). The limits $\omega_{\min }$ and $\omega_{\max }$ explicitly denote frequency cutoffs, e. g. those emerging naturally from the finite bandwidth in lattice systems.

In the limit $T \rightarrow 0$, the Fermi function converges to the Heaviside function, giving

$$
n_{j}=\int_{\omega_{\min }}^{\mu} d \omega \rho_{j}(\omega)
$$

Alternatively, $n_{j}$ can be obtained from a flow equation. To this end an additional source term $\phi_{j} n_{j}$ is added to the action $S$ [ZJ89, p. 148] and the density response vertex is defined as

$$
R_{j}^{\Lambda}:=\left.\partial_{\phi_{j}} \Sigma^{\Lambda}\right|_{\phi_{j}=0}
$$

Note that the independence of frequency stems from the fRG-approximation scheme, cf. subsection 3.1.2. Within this scheme the flow equations for $R_{j}^{\Lambda}$ read

$$
\begin{gathered}
\partial_{\Lambda} R_{j}^{\Lambda}(l, l)=-\frac{U^{\Lambda}}{\pi} \sum_{l^{\prime}=1}^{N} \sum_{r= \pm 1} \sum_{r^{\prime}=-1}^{1} \mathfrak{R e}\left(\tilde{\mathcal{G}}^{\Lambda}\left(l+r, l^{\prime}, \imath \hat{\Lambda}\right) R_{j}^{\Lambda}\left(l^{\prime}, l^{\prime}+r\right) \tilde{\mathcal{G}}^{\Lambda}\left(l^{\prime}+r^{\prime}, l+r, \imath \hat{\Lambda}\right)\right) \\
\partial_{\Lambda} R_{j}^{\Lambda}(l, l \pm 1)=\frac{U^{\Lambda}}{\pi} \sum_{l^{\prime}=1}^{N} \sum_{r^{\prime}=-1}^{1} \mathfrak{R e}\left(\tilde{\mathcal{G}}^{\Lambda}\left(l, l^{\prime}, \imath \hat{\Lambda}\right) R_{j}^{\Lambda}\left(l^{\prime}, l^{\prime}+r^{\prime}\right) \tilde{\mathcal{G}}^{\Lambda}\left(l^{\prime}+r^{\prime}, l \pm 1, \imath \hat{\Lambda}\right)\right)
\end{gathered}
$$

and feed into the flow equation

$$
\partial_{\Lambda} n_{j}^{\Lambda}=-\frac{1}{\pi} \sum_{l=1}^{N} \sum_{r=-1}^{1} \tilde{\mathcal{G}}(l+r, l, \imath \hat{\Lambda}) R_{j}^{\Lambda}(l, l+r)
$$


Further details are given in [Ens05, p. 71 et seq.] and $\left[\mathrm{AEM}^{+} 04\right]$.

Within an exact treatment, the approaches (3.21) and (3.23) would provide identical results, however, within the fRG-approximation scheme used here, the question arises, whether one approach is superior considering a given problem. This discussion is being postponed to chapter 5 , in which the calculation of $n_{j}$ is a dominant issue. 


\section{conductance of Luttinger-liquid wires}

Parts of the results presented in this chapter have been published in WMS09.

\section{summary}

In this chapter, a detailed account of the temperature dependence of the conductance of a quantum wire attached to non-interacting leads is given. Starting from the rather well studied wire coupled at its ends to the end of the leads, the coupling geometry is gradually made more complex and the influence of the geometrical features on the conductance is described and explained.

In the introductory part, the technique to include two-dimensional leads in the approximate fRG, cf. chapter 3, is established.

In section 4.1 the case of one-dimensional leads is studied and the importance of the length scales introduced by overhanging parts of wire and leads is identified and explained. In their presence, the conductance-vs.-temperature curve splits up into temperature regimes showing different (scaling) behavior separated by extended crossover regions. The crossover scales are set by the inverse length of the respective overhanging part.

The subsequent section 4.2 studies a wire coupled over an extended coupling region to two-dimensional leads. The results of section 4.1 are found to persist in the case of symmetric widths of the coupling regions and locally homogeneous coupling strengths as well as for arbitrary widths and locally varying coupling strengths.

In the last side note, the inclusion of spin is discussed.

As discussed in the introduction, cf. section 1.1, the temperature dependence of the conductance $G(T)$ through a quantum wire coupled at its ends to the end of one-dimensional leads is rather well understood. This case constitutes the limit $N_{L / R}^{\text {con }}=1$ and $N_{L / R}^{\text {over }}=N_{L / R}^{\text {lead }}=0$ of the formalism below. The most elementary understanding of the temperature dependence can be obtained using arguments in the spirit of Fermi's Golden Rule [BF04, p. 86]. It states that the conductance of two weakly coupled systems is proportional to the local density of states at both sides of the coupling. This implies, combined with the basic scaling of the density of states at the edge of a semi-infinite Luttinger liquid, cf. section 2.1, that the conductance for tunneling from a Fermi liquid lead into the edge of a Luttinger liquid scales as $G(T) \sim T^{\alpha_{\text {end }}}$. For a wire connected to two leads, it seems intuitive to simply add the two resistances resulting from the two wire-lead couplings, or in other words, apply "Kirchhoff's law" and add up the inverse conductances. Within a microscopic modeling of the semi-infinite leads (given by (2.8)) and the wire (given by (2.5)) 
it was shown, that this reasoning holds for weak couplings $t_{L / R}^{\text {con }} \ll 1$ and long wires $N \gg 1$ in the temperature regime $\pi v_{F} / N \ll T \ll B$ [EMA ${ }^{+} 05$, JMSE07] 1 . The linear conductance thus scales as $G(T) \sim T^{\alpha_{\text {end }}}$ in this regime. The scale $\pi v_{F} / N$ forms a lower bound for any power-law scaling [KF92a, KF92b, KF92c] and the bandwidth $B$ constitutes the (model-specific) upper bound for any scaling, cf. [EMA ${ }^{+}$05, fig. 14] or fig. 4.7 [green, dashed line] for exemplary curves. Note, that the conductance for tunneling from a Fermi liquid lead into the bulk of a Luttinger liquid scales, following Fermi's Golden Rule, as $G(T) \sim T^{\alpha_{\text {bulk }}}$.

In this chapter, the general Hamiltonian (whose constituents were introduced in section 2.2

$$
H=H^{\text {wire }}+h_{L}^{\text {coupl }}+h_{R}^{\text {coupl }}+h_{L}^{1 \mathrm{~d} / 2 \mathrm{~d}-\text { lead }}+h_{R}^{1 \mathrm{~d} / 2 \mathrm{~d}-\text { lead }}
$$

of a wire coupled arbitrarily to one- respectively two-dimensional leads is studied. Lead and wire are chosen to be at half-filling. The structure of the wire-lead couplings is further specified by choosing the coupling matrix 2.9 to be

$$
\begin{aligned}
V_{L}^{\left(N_{1}+l_{x},\left(l_{x}, N_{L}^{\text {lead }}+1\right)^{\mathrm{T}}\right)} & =t_{L}^{l_{x}, \text { coupl }} \\
V_{R}^{\left(N_{3}+l_{x},\left(l_{x}, N_{R}^{\text {lead }}+1\right)^{\mathrm{T}}\right)} & =t_{R}^{l_{x}, \text { coupl }}
\end{aligned}
$$

for $l_{x}=1, \ldots, N_{L / R}^{\text {con }}$ and zero otherwise. Fig. 4.1 sets the notation and illustrates the situation: ${ }^{2}$ The sites of the wire (black bars) are coupled via a hopping term $t_{a}^{j \text {,coupl }}$ to the sites of the leads (green crosses) they lie on top of. For simplicity, the hoppings in the leads are assumed to equal the hopping in the wire, which is used as energy scale, i. e. $t_{a, y}=t_{a, x}=t=1$. The focus lies on low-transmittance contacts $t_{a}^{j \text {,coupl }} \ll 1$. The case of one-dimensional leads is obtained by setting $N_{L / R}^{\text {con }}=1$.

In the approximate fRG, the flow equations of the self-energy, 3.14a and (3.14b), involve the computation of the elements of the tridiagonal part of the full resolvent $\tilde{\mathcal{G}}$. Furthermore, for calculating the conductance 3.19$)$, the elements $\left\langle j^{\prime}|\overline{\mathcal{G}}| j\right\rangle$ with $j^{\prime} \in\left\{N_{1}+1, \ldots, N_{2}\right\}$ and $j \in\left\{N_{3}+1, \ldots, N_{4}\right\}$ are needed as follows from inserting the specific geometry chosen, (4.2a) and (4.2b), into (E.4). The structure of the $(N \times N)$-matrix of $\tilde{\mathcal{G}}^{-1}$ and $\overline{\mathcal{G}}^{-1}$ resulting after projecting the leads using $(3.16)$ is shown in figure C.1 in appendix C. The spatial extension of the coupling area of the two-dimensional leads introduces two full matrices (block 2 and 4). For the inversion involved in the calculation of the wanted elements highly efficient algorithms are available allowing for the treatment of wire lengths reaching the asymptotic regime of Luttinger-liquid behavior, cf. appendix C.

Before turning to the results, the role of length scales and dephasing in mesoscopic systems is briefly being discussed in the following side note. The phenomena

\footnotetext{
${ }^{1}$ Note, however, that this can not be extended to more than two impurities (e. g. two contacts to leads plus one isolated impurity), cf. JMSE07.

${ }^{2}$ If $N_{L / R}^{\text {over }}=0$, the wire is said to couple at its end(s) to the leads. Any $N_{L / R}^{\text {over }}>0$ will be referred to as coupling into the bulk of the wire. The notions 'end-coupled' and 'bulk-coupled' follow this nomenclature. Moreover the wire is said to be coupled to the end(s) of the lead(s) only if $N_{L / R}^{\text {lead }}=0$.
} 


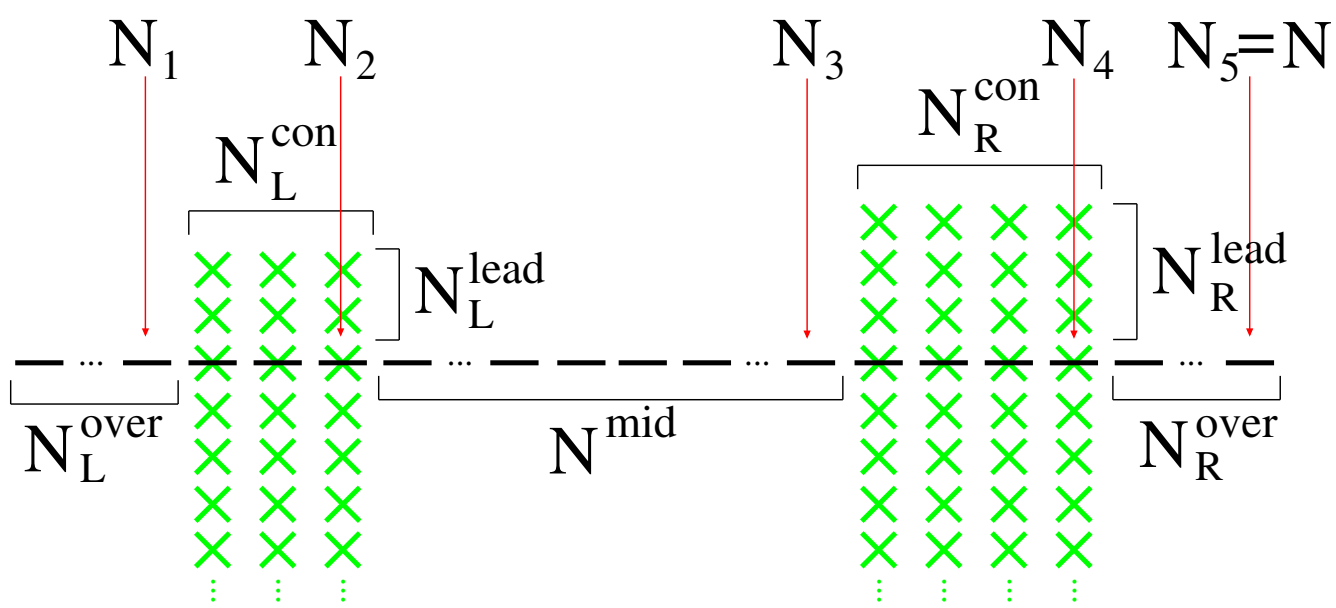

Figure 4.1: Sketch of system geometry considered. The sites of wire and lead lying on top of each other are coupled via a hopping term, cf. text. The length scales are indicated as used throughout this chapter. The total length of the wire is $N=N_{5}=$ $N_{L}^{\text {over }}+N_{R}^{\text {over }}+N_{L}^{\text {con }}+N_{R}^{\text {con }}+N^{\text {mid }}$.

described above entirely in the language of temperature or energy scales can equivalently be described in this language of length scales. Both languages are used in parallel in subsequent sections.

\section{side note: length scales in mesoscopic systems}

The regime of mesoscopic physics can be characterized by a certain order of length scales, cf. e. g. [BF04, p. 103]. For the specific model used in this chapter (cf. introductory part), the ordering reads

$$
a_{0}=1<\lambda_{F} \lesssim N_{B} \ll \underbrace{\lesssim\left\{N_{L / R}^{\text {over }}, N_{L / R}^{\text {lead }}, N\right\}}_{\text {geometry-dependent }} \ll N_{\phi} \lesssim N_{\text {in }} .
$$

The two lower bounds, viz. atomic scale $a_{0}$ and Fermi wave length $\lambda_{F}$, are fixed by the choice of $a_{0}$ to be the lattice spacing (thus $a_{0}=1$ ) and the choice of half-filling, respectively. The two upper bounds, viz. the phase and energy-relaxation length $N_{\phi}$ and $N_{\text {in }}$, are essentially infinite. This stems from the fact, that all features added to the clean wire are static, cf. e. g. [Dat95, p. 19], no inelastic processes are included, and all inelastic and dephasing processes from the electron-electron interaction, cf. e. g. [Imr97, p. 56], are excluded in the approximate fRG, cf. section 3.1.2. The thermal length $N_{T}$ is defined as the distance beyond which the information on the initial phase of an electron is lost due to finite temperature,viz.

$$
N_{T}=\frac{\pi v_{F}}{T}
$$

For the model-specific band width $B$, an analogous length $N_{B}=\pi v_{F} / B$ is defined. 
Within this language of length scales, the temperature regime $\pi v_{F} / N \ll T \ll B$ in which power-law scaling in $G(T)$ is observed for an end-coupled wire (cf. introductory paragraph) reads $N \gg N_{T} \gg N_{B}$, i. e. the thermal length is smaller than the overall length of the system.

Concerning the new energy scales introduced by the overhanging parts of the wire, the description in terms of temperature and energy scales is equivalent to the description in terms of thermal length and lengths of the overhanging parts.

\section{quantum wire with arbitrarily coupled one-dimensional leads}

One-dimensional leads, i. e. $N_{L / R}^{\text {con }}=1$, are connected at an arbitrary site to an arbitrary site of the wire. Following fig. 4.1, the coupling sites in the wire are $N_{L}:=N_{1}+1$ and $N_{R}:=N_{3}+1$, those in the leads $N_{L}^{\text {lead }}+1$ and $N_{R}^{\text {lead }}+1$. The site index $l_{x}$ of the coupling strengths $t_{L / R}^{l_{x} \text {,coupl }}$ is redundant and will be omitted.

\section{non-interacting electrons, analytic approximation}

Each of the coupling sites can either be even or odd, just as the overall length $N$ of the wire. For $T \rightarrow 0$, this leads to pronounced even-odd effects, as, according to $(3.19)$, the conductance at vanishing temperature is given by the effective transmission E.5 at the Fermi energy. This vanishes for even coupling sites, since the local spectral function at the Fermi energy is zero for even sites in the finite and semi-infinite hopping chain, thus allowing for a nonvanishing conductance only if all coupling sites are odd. Furthermore, if, for odd coupling sites and symmetric coupling $t_{L}^{\text {coupl }}=t_{R}^{\text {coupl }}$, the length $N$ is chosen to be odd, $G(T \rightarrow 0) / G_{0} \rightarrow 1$, cf. Ogu99, $\mathrm{EMA}^{+}$05]. These even-odd effects disappear with increasing temperature $T>\pi v_{F} / N$. To avoid confusions stemming from these effects, the results presented in the following are obtained using systems of odd length and odd coupling sites. Thus, $G(T \rightarrow 0) / G_{0} \rightarrow 1$ for symmetric coupling $t_{L}^{\text {coupl }}=t_{R}^{\text {coupl }}$. This still holds in the interacting systems of the next subsection.

Figure 4.2 compares the conductance $G(T)$ of a non-interacting wire coupled to leads at its ends with a wire coupled to leads in its bulk. The coupling is weak $\left(t_{L}^{\text {coupl }}=t_{R}^{\text {coupl }}=0.25\right)$ and there are no overhanging parts of the lead, i. e. $N_{L / R}^{\text {lead }}=0$. For the end-coupled case $N_{L / R}^{\text {over }}=0$, while for the bulk-coupled case the wire has overhanging parts $N_{L}^{\text {over }}=20, N_{R}^{\text {over }}=286$. The conductance of the end-coupled wire [solid, black line] starts $G_{0}$ for $T=0$, crosses over into a plateau at $T \approx \pi v_{F} / N$ [left vertical line] and eventually falls off as $T^{-1}$ for $T \gg B$, cf. [EMA ${ }^{+} 05$ ]. For the system with overhanging parts, the plateau splits into three clearly distinguishable regimes with the two additional crossover scales $\pi v_{F} / N_{L}^{\text {over }}$ and $\pi v_{F} / N_{R}^{\text {over }}$ [red, dashed line], indicated by the two vertical lines to the right. In the first regime $\pi v_{F} / N \ll T \ll$ $\pi v_{F} / N_{R}^{\text {over }}$ a plateau with the same conductance as for the end-coupled wire appears. 


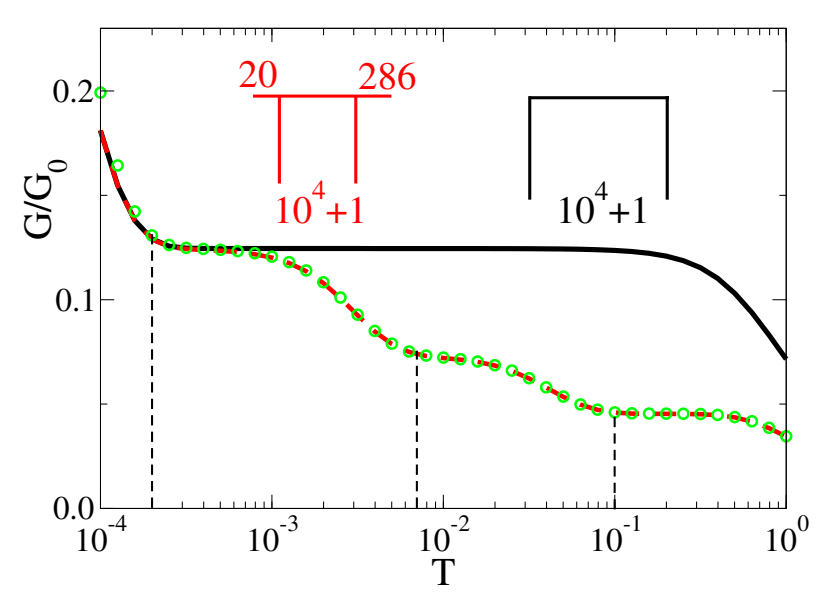

Figure 4.2: Conductance $G$ as function of temperature $T$ of a noninteracting wire $(U=0)$ of length $N=$ $10^{4}+1$ and coupling $t_{L}^{\text {coupl }}=t_{R}^{\text {coupl }}=$ 0.25 with $N_{L / R}^{\text {over }}=0$ [black, solid] (setup shown in right inset) respectively $N_{L}^{\text {over }}=20, N_{L}^{\text {over }}=286[$ red, dashed] (setup shown in left inset), both obtained from $(3.19)$. Green circles: same parameters as red line, calculated with the approximation 4.6. Vertical lines indicate the crossover scales.

At the two additional scales, the conductance crosses over to new, lower plateaus. Eventually, for $T \gg B$, the conductance falls off proportional to $T^{-1}$.

For $\pi v_{F} / N \ll T \ll B$ and $t_{L / R}^{\text {coupl }} \ll 1,|t(\varepsilon)|^{2}$ basically consists of $N$ well separated Lorentz peaks, located approximately at the eigenenergies $\varepsilon_{\alpha}$ of the isolated wire. Generalizing the calculation in $\left[\mathrm{EMA}^{+} 05\right]$, the integrated weight $w_{\alpha}$ of the Lorentzian resonance near $\varepsilon_{\alpha}$ is

$$
w_{\alpha} \approx 4 \pi\left[\frac{1}{\Delta_{L, \alpha}}+\frac{1}{\Delta_{R, \alpha}}\right]^{-1} .
$$

Here the partial widths $\Delta_{a, \alpha}$ are given by

$$
\Delta_{a, \alpha}=\left(t_{a}^{\text {coupl }}\right)^{2} \frac{2}{(N+1) \sin \left[k\left(\varepsilon_{\alpha}\right)\right]} \sin ^{2}\left[N_{a} k\left(\varepsilon_{\alpha}\right)\right] \sin ^{2}\left[\left(N_{a}^{\text {lead }}+1\right) k\left(\varepsilon_{\alpha}\right)\right],
$$

with $N_{a}$ the site of the wire to which lead $a$ couples. Note that $\sin ^{2}\left[N_{L} k\left(\varepsilon_{\alpha}\right)\right]=$ $\sin ^{2}\left[\left(N_{L}^{\text {over }}+1\right) k\left(\varepsilon_{\alpha}\right)\right]$ by definition and $\left.\sin ^{2}\left[N_{R} k\left(\varepsilon_{\alpha}\right)\right]=\sin ^{2}\left[\left(N_{R}^{\text {over }}+1\right) k\left(\varepsilon_{\alpha}\right)\right]\right]^{3}$ In the temperature regime $\pi v_{F} / N \ll T \ll B$ the Landauer-Büttiker formula simplifies to

$$
G(T) / G_{0} \approx \sum_{\alpha=1}^{N} w_{\alpha}\left(-\frac{\partial f}{\partial \varepsilon}\right)_{\varepsilon=\varepsilon_{\alpha}} .
$$

The overhanging parts of the wire and those of the leads enter symmetrically in (4.6). Thus, the choice $N_{L}^{\text {lead }}=20, N_{R}^{\text {lead }}=286$ and $N_{L / R}^{\text {over }}=0$ is equivalent to $N_{L}^{\text {over }}=20, N_{R}^{\text {over }}=286$ and $N_{L / R}^{\text {lead }}=0$ and gives the same conductance as the one shown in fig. 4.2. This equivalence is broken in an interacting wire.

All four possible new scales, viz. $\pi v_{F} / N_{L / R}^{\text {lead }}$ and $\pi v_{F} / N_{L / R}^{\text {over }}$, can be resolved by applying the analytical approximation (4.6) to a very long wire. This implies a very

\footnotetext{
${ }^{3}$ Explicitely: $\sin \left[N_{R} k\left(\varepsilon_{\alpha}\right)\right]=\sin \left[\left(N+1-\left(N_{R}^{\text {over }}+1\right)\right) k\left(\varepsilon_{\alpha}\right)\right]=\sin \left[(N+1) k\left(\varepsilon_{\alpha}\right)\right] \cos \left[\left(N_{R}^{\text {over }}+\right.\right.$ 1) $\left.k\left(\varepsilon_{\alpha}\right)\right]-\cos \left[(N+1) k\left(\varepsilon_{\alpha}\right)\right] \sin \left[\left(N_{R}^{\text {over }}+1\right) k\left(\varepsilon_{\alpha}\right)\right]$. With $k\left(\varepsilon_{\alpha}\right)=\frac{\pi n_{\alpha}}{N+1}$ and $n_{\alpha} \in\{1, \ldots, N\}$ follows: $\sin \left[(N+1) k\left(\varepsilon_{\alpha}\right)\right]=0$ and $\cos \left[(N+1) k\left(\varepsilon_{\alpha}\right)\right]= \pm 1$ and thus $\sin \left[N_{R} k\left(\varepsilon_{\alpha}\right)\right]= \pm \sin \left[\left(N_{R}^{\text {over }}+1\right) k\left(\varepsilon_{\alpha}\right)\right]$ or $\sin ^{2}\left[N_{R} k\left(\varepsilon_{\alpha}\right)\right]=\sin ^{2}\left[\left(N_{R}^{\text {over }}+1\right) k\left(\varepsilon_{\alpha}\right)\right]$.
} 

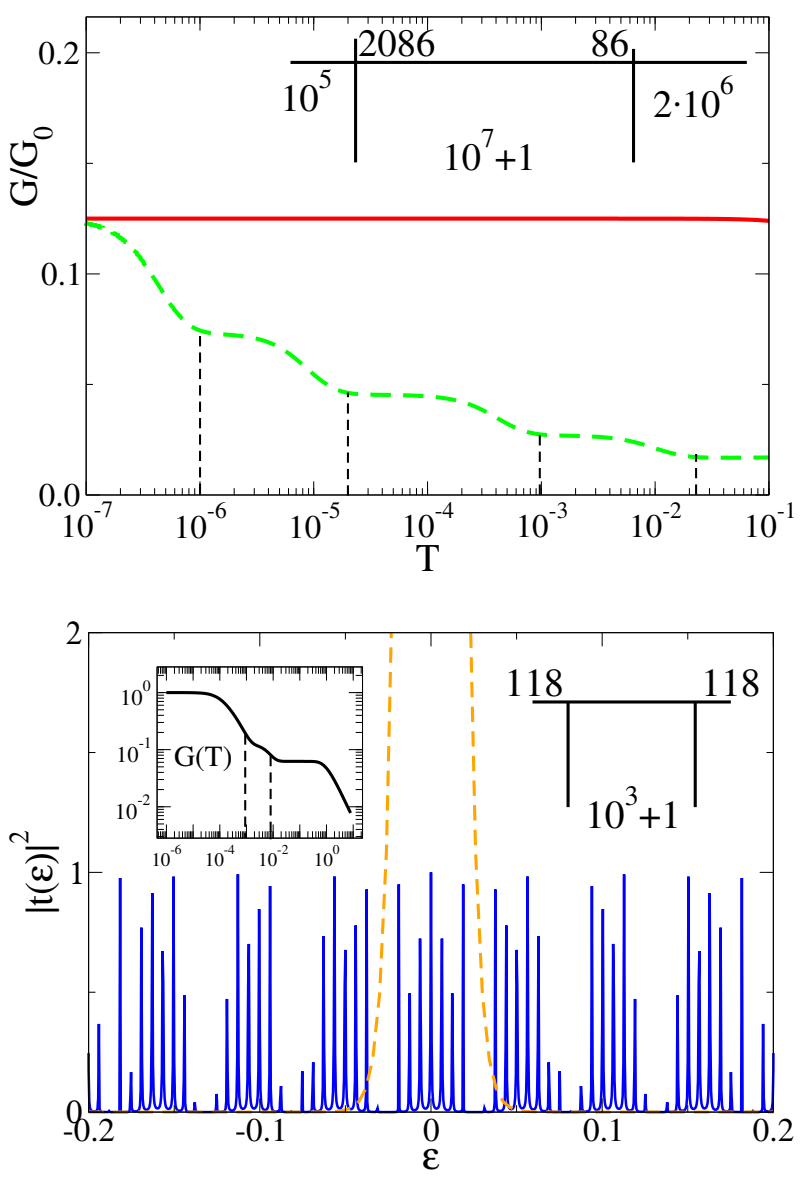

Figure 4.3: Conductance $G$ as function of temperature $T$ of a noninteracting wire of length $N=10^{7}+1$ and coupling $t_{L}^{\text {coupl }}=t_{R}^{\text {coupl }}=0.25$ with $N_{L / R}^{\text {lead }}=N_{L / R}^{\text {over }}=0$ [red, solid] respectively $N_{L}^{\text {lead }}=2086, N_{R}^{\text {lead }}=86$, $N_{L}^{\text {over }}=10^{5}$ and $N_{R}^{\text {over }}=2 \cdot 10^{6}$ [green, dashed] as depicted in inset. Both curves were obtained with the approximation (4.6). Vertical lines indicate the crossover scales.

Figure 4.4: Transmission amplitude $|t(\varepsilon)|^{2}$ for $T=0.005$ of system of length $N=1001$ coupled such that $N_{L}^{\text {over }}=N_{R}^{\text {over }}=118$ [blue, solid line]; $(-\partial f / \partial \varepsilon)$ for $T=0.005$ [orange, dashed line]; inset: conductance through system as function of $T$.

broad interval $\pi v_{F} / N \ll B$. Figure 4.3 shows the resulting conductance [green, dashed line] in comparison to an end-coupled wire of the same length [red, solid line].

A basic understanding of the appearance of additional length scales and the associated conductance plateaus can be obtained by investigating the kernel $(-\partial f / \partial \varepsilon)$ $|t(\varepsilon)|^{2}$ in the Landauer-Büttiker formula (3.19). Fig. 4.4 shows the transmission amplitude $|t(\varepsilon)|^{2}$ for a wire of length $N=10^{3}+1$, bulk-coupled to the end of the leads with $N_{L}^{\text {over }}=N_{R}^{\text {over }}=118$. The equal length of the overhanging parts implies that only two crossover scales exist, viz. $\pi v_{F} / N$ and $\pi v_{F} / N^{\text {lead }}:=\pi v_{F} / N_{L}^{\text {lead }}=$ $\pi v_{F} / N_{R}^{\text {lead }}$, which is chosen for the sake of readability. The transmission amplitude $|t(\varepsilon)|^{2}$ shows $N$ clearly distinguishable Lorentz-shaped peaks (only a small part of the band is shown in the figure), one of which lies at $\varepsilon=0$ as $N$ is odd. The periodic overstructure visible in the distribution of the peaks stems from the length scale $N^{\text {lead }}$ entering the transmission amplitude in the standing waves $\sin ^{2}\left(\left(N_{L / R}^{\text {lead }}+1\right) k\right)$ (cf. (4.4) and (4.5)), which in this case yields $|t(\varepsilon)|^{2} \sim \sin ^{4}\left(\left(N^{\text {lead }}+1\right) k\right)$. An additional example for this overstructure is provided in fig. 4.5 for a very small system and unequal overhanging parts. Returning to fig. 4.4, the left inset shows the conductance with its two plateaus, which can be understood by scanning $|t(\varepsilon)|^{2}$ with the derivative of the Fermi function as do (3.19) and (4.6). For $T \rightarrow 0,(-\partial f / \partial \varepsilon) \rightarrow \delta(\varepsilon)$ and, thus, only the central peak of height one enters $G(T)$ and $G(T \rightarrow 0) \rightarrow G_{0}$. 
Figure 4.5: Transmission amplitude $|t(\varepsilon)|^{2}$ for $T=0.005$ of system of length $N=25$ coupled such that $N_{L}^{\text {over }}=2$ and $N_{R}^{\text {lead }}=8$ [red]; blue line: $\sin ^{2}[3 k(\varepsilon)] \sin ^{2}[9 k(\varepsilon)]$. Note the logarithmic y-axis.
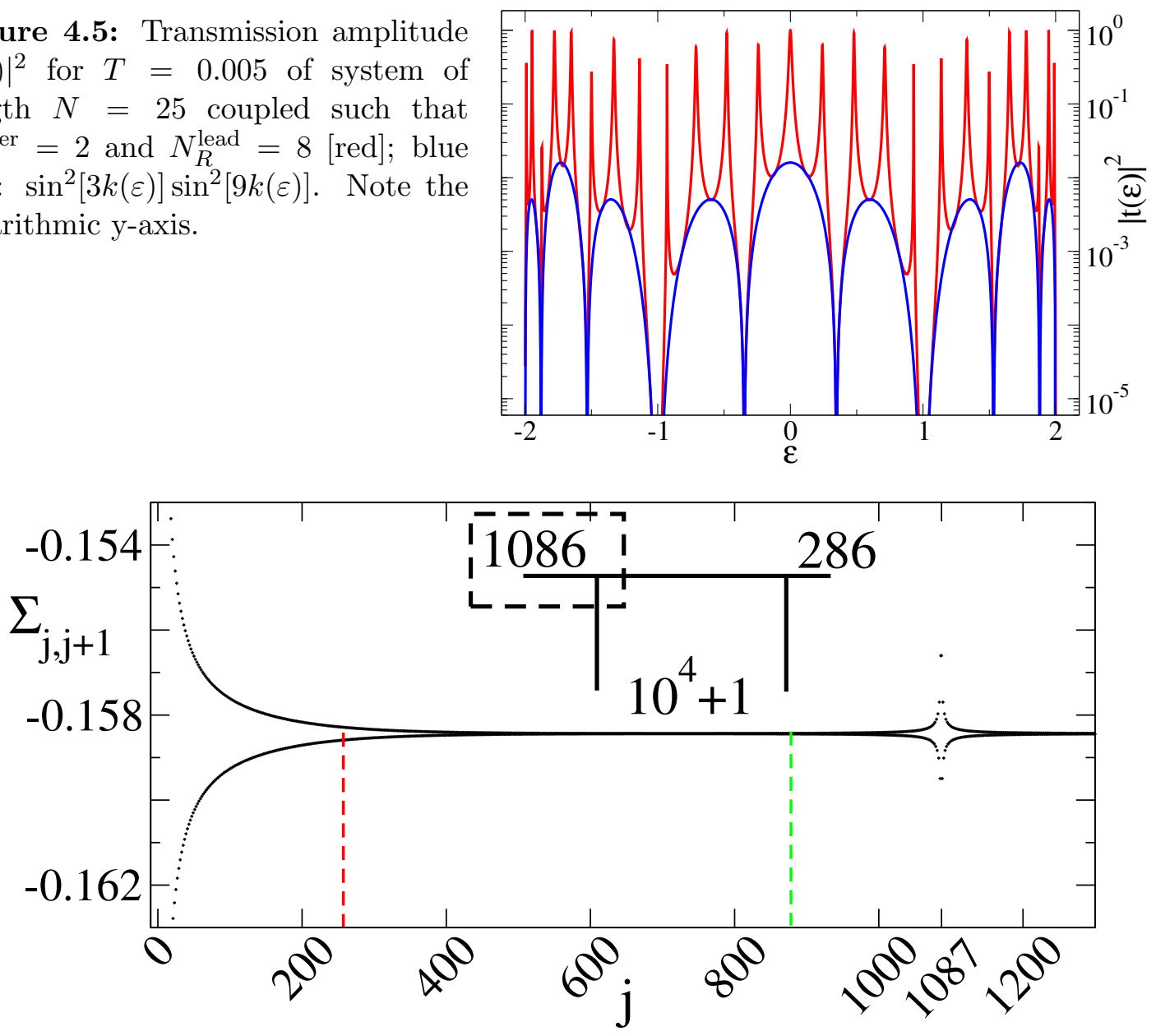

Figure 4.6: Off-diagonal self-energy at the end of the fRG flow for an interacting wire of length $N=10^{4}+1$ with $U=0.5$ and couplings $t_{R}^{\text {coupl }}=t_{L}^{\text {coupl }}=0.25$ at $T=4 \cdot 10^{-3}$. The leads are attached with their end (i. e. $N_{L / R}^{\text {lead }}=0$ ) to the bulk of the wire with $N_{L}^{\text {over }}=1086$ and $N_{R}^{\text {over }}=286$. The part of the self-energy shown in the main plot is indicated by the dashed box in the inset. Long-ranged oscillations emerge from the boundaries and from the coupling site. The respective scale $j_{T}$ beyond which the oscillations decay exponentially is indicated by the vertical dashed lines.

For $T \gg \pi v_{F} / N,(-\partial f / \partial \varepsilon)$ becomes broader than the central peak and averages over the first few peaks of the overstructure leading to the first plateau in $G(T)$. Next, for $T \gg \pi v_{F} / N^{\text {lead }},(-\partial f / \partial \varepsilon)$ becomes broader than the central peak of the overstructure, averaging over the gap between the neighboring peaks, leading to the second, lower plateau. Eventually the average extends over the entire band $\left(N_{T}>N_{B}\right)$ and leads to the $T^{-1}$ behavior. The same reasoning holds for a larger number of length scales, leading to a more complicated overstructure in $|t(\varepsilon)|^{2}$.

\section{interacting electrons}

The approximate fRG flow leads to a static self-energy for the interacting wire, which is tridiagonal in position space. cf. section 3.1.2. At half-filling and for tun- 


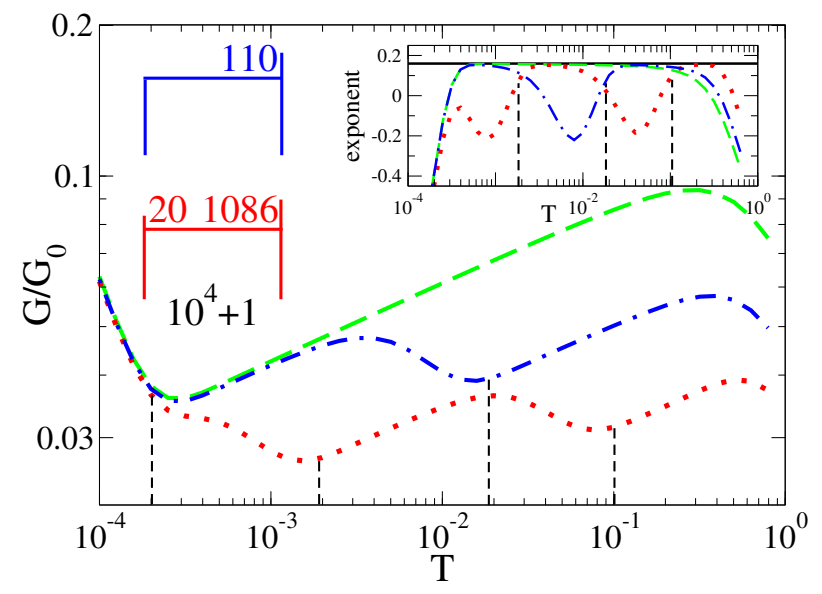

Figure 4.7: Conductance $G$ as function of temperature $T$ of an interacting wire of length $N=10^{4}+1$ with $U=0.5$. The ends of the wire are weakly coupled $\left(t_{L}^{\text {coupl }}=t_{R}^{\text {coupl }}=\right.$ $0.25)$ to leads with overhanging parts of length $N_{L / R}^{\text {lead }}=0$ [green, dashed], $N_{L}^{\text {lead }}=0, N_{R}^{\text {lead }}=110$ [blue, dashdotted] and $N_{L}^{\text {lead }}=20, N_{R}^{\text {lead }}=$ 1086 [red, dotted]. The setups are sketched in the left insets. The vertical dashed lines terminating at the different curves indictate the corresponding crossover scales. Right inset: Effective exponents of the conductance curves. Solid horizontal line indicates the fRG approximation for $\alpha_{\text {end }}$, cf. [EMA $\left.{ }^{+} 05\right]$.

nel barriers, the diagonal part vanishes and the effective potential is given by the non-local term, viz. the modulation $\Sigma_{j, j+1}$ of the hopping. If the leads are coupled to the end of the wire (with or without featuring overhanging parts themselves), each contact barrier is the source of such a non-local potential, oscillating with the distance $\Delta j$ from the end (site 1 and site $N$, respectively) as $(-1)^{\Delta j}$ and decaying as $1 / \Delta j$. The prefactor of the decay is universal in the sense that it does not depend on the strength of the tunnel barriers $t_{L}^{\text {coupl }}$ and $t_{R}^{\text {coupl }}$, even in the limiting case of open boundaries $\left(t_{L / R}^{\text {coupl }}=0\right.$, i. e. an isolated wire), cf. [AEM ${ }^{+} 04$. For finite temperature, the power-law decay is cut off at a scale $j_{T} \sim 1 / T$, which marks the crossover from power-law to exponential decay of $\Sigma_{j, j+1}$, cf. [EMA $\left.{ }^{+} 05\right]$. Scattering off this effective potential leads to the power-law scaling of the local spectral function (cf. e. g. [MMSS02a]) and the conductance with exponents determined by the universal prefactor $\left[\mathrm{EMA}^{+}\right.$05, YGM94].

A typical off-diagonal component $\Sigma_{j, j+1}$ of the self-energy of an interacting wire with two overhanging parts $\left(N_{L}^{\text {over }}=1086\right.$ and $\left.N_{R}^{\text {over }}=286\right)$, weakly coupled to the leads $\left(t_{R}^{\text {coupl }}=t_{L}^{\text {coupl }}=0.25\right)$, is shown in fig. 4.6. From each end of the wire $(-1)^{\Delta j} / \Delta j$-oscillations with universal prefactor emerge and become exponentially damped at distance $\Delta j=j_{T}$ (indicated by the red vertical line). To enhance readability only one end is shown in the main plot as indicated in the inset. The coupling to the lead (at site 1087) introduces similar oscillations, however, with a nonuniversal prefactor. In contrast to the situation found close to an end-contact, these oscillations do not lead to a power-law suppression of the local spectral function close to the coupling site. Furthermore, as explained in section 3.1.2, the approximate fRG procedure does not capture bulk Luttinger-liquid power laws with exponents of order $U^{2}$. This implies that the local spectral function close to the coupling site in the bulk does not show any power law at all. 
Figure 4.8: As in fig. 4.7 but with $N_{L / R}^{\text {lead }}=0, N_{R}^{\text {over }}=0$ and for $N_{L}^{\text {over }}=0$ [green, dashed], $N_{L}^{\text {over }}=10$ [red, dotted], $N_{L}^{\text {over }}=110$ [cyan, dash-doubledotted], $N_{L}^{\text {over }}=1110$ [blue, dashdotted].

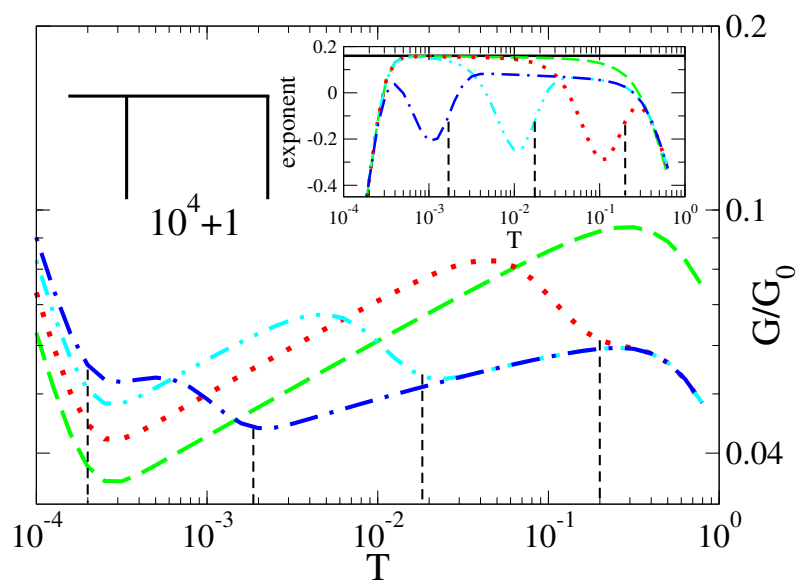

The conductance for several setups is shown in figs. 4.7 to 4.9 for $T>\pi v_{F} / N$, i. e. in a regime in which the thermal length is shorter than the overall length of the system and the plateaus emerge in the non-interacting case. The length of the wire is $N=10^{4}+1$, which corresponds to roughly a micrometer taking typical lattice constants. The vertical dashed lines terminating at the different curves indicate the energy scales induced by the respective length scales of the different setups. The curve for the wire coupled at its ends to the ends of the leads is included for comparison [green, dashed lines]. The inset on the right-hand side of each figure shows the effective exponent of the curves $G(T)$, computed as their logarithmic derivative. Finally, the remaining insets depict sketches of the setups studied.

Fig. 4.7 focuses on wires coupled at their ends, viz. $N_{L / R}^{\text {over }}=0$. For all three temperature regimes,

$$
\begin{array}{r}
\pi v_{F} / N \ll T \ll \pi v_{F} / \max \left(N_{L / R}^{\text {lead }}\right) \\
\pi v_{F} / \max \left(N_{L / R}^{\text {lead }}\right) \ll T \ll \pi v_{F} / \min \left(N_{L / R}^{\text {lead }}\right) \\
\pi v_{F} / \min \left(N_{L / R}^{\text {lead }}\right) \ll T \ll B,
\end{array}
$$

the power law $G(T) \sim T^{\alpha_{\text {end }}}$ is found. Note that for the system with $N_{L}^{\text {lead }}=$ $20, N_{R}^{\text {lead }}=1086$ [red, dotted line], the first temperature regime $\pi v_{F} / N \ll T \ll$ $\pi v_{F} / N_{R}^{\text {lead }}$ is too small for a proper plateau in the effective exponent to develop. However, by reducing $N_{R}^{\text {lead }}$ this regime is extended, such that the effective exponent trends towards $\alpha_{\text {end }}$.

This simple case of a system with no overhanging parts of the wire (in other words, the particles tunnel into the end of the interacting wire) demonstrates a central result of this chapter. The power law, which for wires of length in the micrometer range, coupled at their ends to the ends of the leads holds over several decades in $T$, is split into significantly smaller temperature regimes, defined by the length scales of the system's geometry, with power-law scaling interrupted by extended crossover regimes. The three plateaus of different conductance identified in the non-interacting case become power laws with identical exponents in the interacting case. For each temperature regime the appearance of these exponents can be traced back to the spatial dependence of the self-energy $\Sigma_{j, j+1}$. Over the entire 


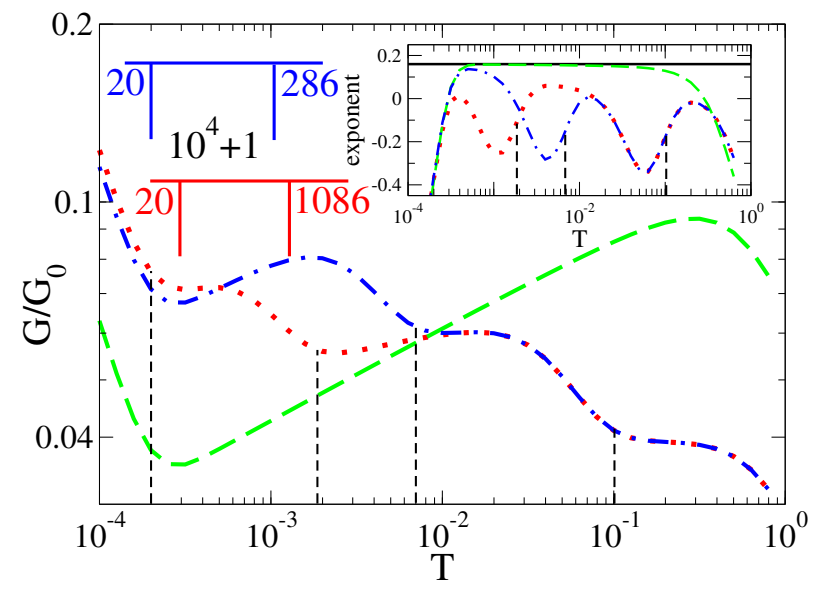

Figure 4.9: As in fig. 4.7 but with $N_{L / R}^{\text {lead }}=0, N_{R}^{\text {over }}=0$ and coupling into the bulk: $N_{L / R}^{\text {over }}=0$ [green, dashed], $N_{L}^{\text {over }}=20, N_{R}^{\text {over }}=286$ [blue, dashdotted], $N_{L}^{\text {over }}=20, N_{R}^{\text {over }}=1086[$ red, dash-dotted].

range $\pi v_{F} / N \ll T \ll B$, particles moving from the left to the right contact have to pass these long-range oscillatory scattering potentials with universal amplitude, originating from each end of the wire. For $\pi v_{F} / N \ll T$ these are well separated, such that the simple arguments presented in the introductory part hold, implying $G(T) \sim T^{\alpha_{\text {end }}}$.

The role of the length scales resulting from overhanging parts is further exemplified in fig. 4.8 on a system with $N_{L / R}^{\text {lead }}=0$ and $N_{R}^{\text {over }}=0$, but varying $N_{L}^{\text {over }}$, in other words, a system with a stub on the left. Starting from a system coupled at the ends, i. e. $N_{L}^{\text {over }}=N_{R}^{\text {over }}=0$ [green, dashed line], the left coupling site is moved into the bulk of the wire. For temperatures $T \ll \pi v_{F} / N_{L}^{\text {over }}$, the oscillatory scattering potential emerging from the left end of the wire extends well over the coupling site (since $1 / T \gg N_{L}^{\text {over }}$ ), therefore a particle has to pass both oscillatory potentials originating from the ends of the wire and the situation of particles tunneling into and out of the end of the wire is essentially recovered. This leads to the power-law scaling of the conductance with exponent $\alpha_{\text {end }}$. With increasing $N_{L}^{\text {over }}$ the crossover scale $\pi v_{F} / N_{L}^{\text {over }}$ decreases and the effective exponent of $\alpha_{\text {end }}$ is obtained inside a narrowing temperature regime (cf. inset on right-hand side). For the system with the largest $N_{L}^{\text {over }}$ shown (i. e. $N_{L}^{\text {over }}=1110$ ) [blue, dash-dotted line], the interval $\left[v_{F} / N, v_{F} / N_{L}^{\text {over }}\right]$ becomes too narrow for the logarithmic derivative to reach its asymptotic value (cf. inset on right-hand side). For temperatures $T \gtrsim \pi v_{F} / N_{L}^{\text {over }}$, the oscillatory potential originating at the left end of the wire is exponentially being damped before reaching the left contact. On their direct path from the left to the right contact, the particles thus merely experience the potential emerging from the right end of the wire. The local spectral function close to the origin of this potential scales as $\omega^{\alpha_{\text {end }}}$. The somewhat natural conclusion, that $G(T)$ follows a power law with exponent $\alpha_{\text {end }}$ in $\pi v_{F} / N_{L}^{\text {over }} \ll T \ll B$, however, is not correct as the exponent seems to approach an asymptotic value significantly smaller than $\alpha_{\text {end }}$ [blue, dashed dotted lines in main plot and inset]. The problem is revisited after the next paragraph.

As a third example systems with $N_{L / R}^{\text {lead }}=0$ (no overhanging parts of the leads) and different positions of the coupling to the wire $N_{L / R}^{\text {over }}$ are shown in fig. 4.9. The 
Figure 4.10: System as in fig. 4.8 with $N_{L}^{\text {over }}=1110$, as shown in the inset. Effective exponents in the regime $\pi v_{F} / N_{L}^{\text {over }} \ll T \ll B$ for different interaction strengths $U$, viz. from bottom to top: $U=0.25,0.5,0.75,1.0,1.5$. The horizontal lines show the fRG approximation for $\alpha_{\text {end }}(U=0.5) / 2$ [magenta] and $\alpha_{\text {end }}(U=1.0) / 2$ [cyan], cf. $\left[\mathrm{EMA}^{+} 05\right]$. The vertical line indicates the crossover scale.

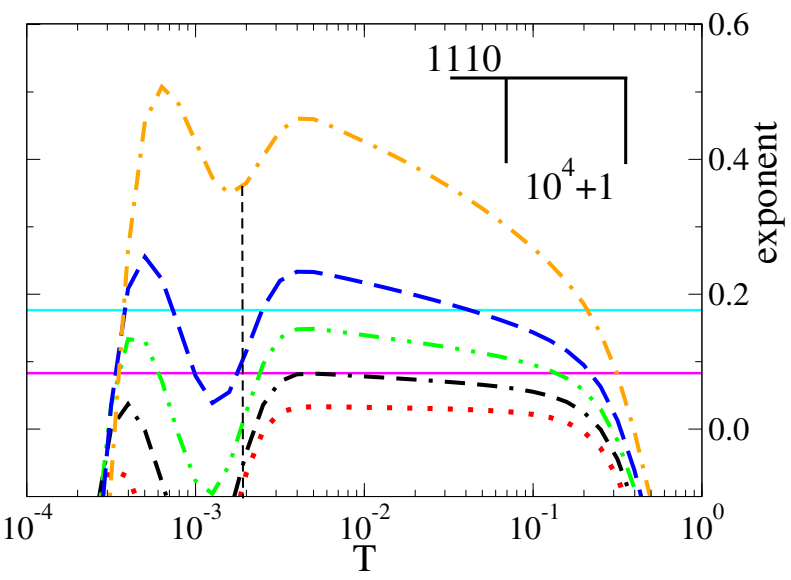

form of the conductance curves can be understood within the reasoning presented above. In the regime $\pi v_{F} / N \ll T \ll \pi v_{F} / \max \left(N_{L / R}^{\text {over }}\right)$ the conductance follows the power law $G(T) \sim T^{\alpha_{\text {end }}}$, because the oscillations of $\Sigma_{j, j+1}$ from the two ends reach well beyond the positions of the contacts. For $N_{R}^{\text {over }}=1086$ [red, dotted line] this regime is too narrow for the power law to be properly developed. For $\pi v_{F} / \max \left(N_{L / R}^{\text {over }}\right) \ll T \ll \pi v_{F} / \min \left(N_{L / R}^{\text {over }}\right)$, the oscillations from the end of the longer overhanging part are readily cut off before reaching the respective contact (the exponential damping sets in at a scale $j_{T} \sim 1 / T$, thus, $j_{T} \ll \max \left(N_{L / R}^{\text {over }}\right)$ ), while the ones emerging from the shorter overhanging part still extend well over the respective contact. In this regime the curves show a behavior similar to the one discussed in the context of fig. 4.8 in the preceding paragraph. In the third regime $\pi v_{F} / \min \left(N_{L / R}^{\text {over }}\right) \ll T \ll B$, the oscillatory potentials from both ends do not reach into the region between the left and right contact and power-law behavior cannot be found (cf. the conductance plateau of the red, dotted and the blue, dash-dotted line in fig. 4.8). This observation is in accordance with the fact that the oscillations of $\Sigma_{j, j+1}$ originating from the contacts do not induce any power-law suppression in the local spectral function in contrast to the ends of the wire. However, the conductance can be expected to show power-law scaling with the bulk Luttinger-liquid exponent $\alpha_{\text {bulk }} \sim U^{2}$ in this temperature regime, which is not being captured by the approximate fRG, cf. section 3.1.2.

In order to obtain a better understanding of the (apparent) exponent smaller than $\alpha_{\text {end }}$ found in the regime $\pi v_{F} / N_{L}^{\text {over }} \ll T \ll B$, fig. 4.10 shows the logarithmic derivative of $G(T)$ in this temperature regime for a system with $N_{L}^{\text {over }}=1110$, $N_{R}^{\text {over }}=0$ and different interaction strengths $U$ (cf. the blue, dash-dotted line in fig. 4.8 for the conductance curve with $U=0.5$ ). For $U \leq 0.5$ [black, double-dashdotted line] a scaling $G \sim T^{\alpha_{\text {end }} / 2}$ seems likely, however, for $U>0.5$, the plateau of the logarithmic derivative becomes increasingly tilted, such that no definite statement about any power law can be made. The horizontal lines indicate the exponents $\alpha_{\text {end }} / 2$ for $U=0.5$ respectively $U=1.0$ calculated within the approximate fRG, cf. $\left[\mathrm{EMA}^{+} 05\right]$. A complementary calculation within the framework of the poor man's RG (cf. section 3.2) suggests that the conductance curves essentially show a complicated logarithmic structure. However, both approaches fail to include bulk 


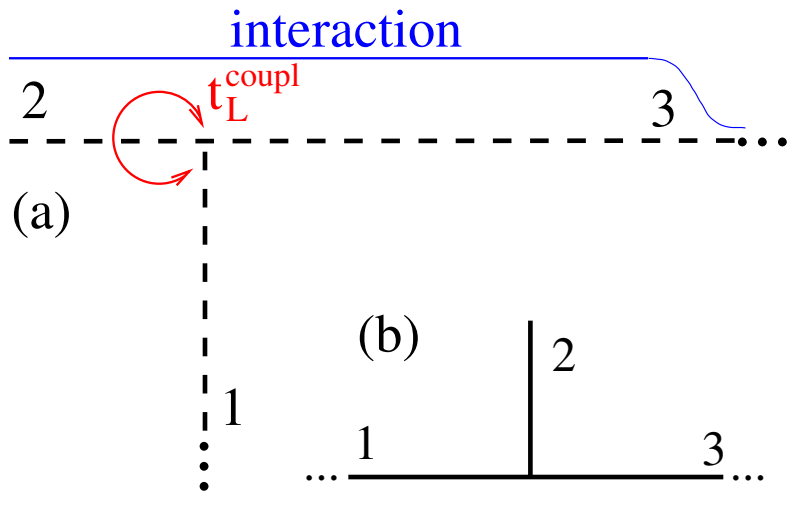

Figure 4.11: Sketches of the systems considered in the side note: (a) System used in fRG, non-interacting lead (1) coupled with $t_{L}^{\text {coupl }}$ into the bulk of the wire with overhanging part $N_{L}^{\text {over }}=1110$. Right end of wire coupled perfectly smooth (cf. text) to right lead. The blue solid line sketches the form of the interaction curve $U_{j, j+1}$. (b) System used in poor man's RG, non-interacting semi-infinite lead (1) connected to the bulk of semi-infinite wire with homogeneous interaction (2) $\&(3)$

Luttinger-liquid behavior, whose proper inclusion is expected to alter the curves $G(T)$ in this temperature regime. Details of the poor man's RG calculation and the exact expressions can be found in the side note at the end of this section.

The conductance through "mixed" systems, i. e. those with overhanging parts of the wire as well as overhanging parts of the leads, exhibits an even richer structure in its temperature dependence. As in the non-interacting case (cf. fig. 4.3) up to five different temperature regimes separated by the corresponding crossover regimes may appear in $\left[\pi v_{F} / N, B\right]$. The behavior well inside these regimes can be understood within the reasoning explained above.

\section{side note: the stub in poor man's RG}

In order to further clarify the behavior of the conductance through a system with a single overhanging part of the wire, i. e. $N_{L / R}^{\text {lead }}=0, N_{R}^{\text {over }}=0$ and $N_{L}^{\text {over }} \neq 0$, cf. fig. 4.8, the somewhat simpler system shown in fig. 4.11 (a) is considered in this side note. By setting the coupling to the right lead $t_{R}^{\text {coupl }}=1$ and switching off the interaction smoothly, viz. $U_{j, j+1}=U \frac{\arctan \left[s\left(N-j-j_{s}\right)\right]-\arctan \left[s\left(1-j_{s}\right)\right]}{\arctan \left[s\left(N / 2-j_{s}\right)\right]-\arctan \left[s\left(1-j_{s}\right)\right]}$ with $j_{s}=56$ and $s=0.25\left[\mathrm{EMA}^{+} 05\right.$ ], a perfect contact to the right lead is obtained, cf. [JMS06] for details. Thereby, for large $N$ (i. e. $N \gtrsim 10^{3}$ ), a semi-infinite wire with homogeneous interaction is modelled and the effect of the coupling into the bulk with overhanging part $N_{L}^{\text {over }}$ is isolated. The system is equivalent to the one shown in fig. 4.11 (b), which can be treated within the poor man's RG introduced in section 3.2, cf. [DRS04]. Using (3.18), the scattering matrix

$$
\mathbf{S}=\left(\begin{array}{lll}
r_{11} & t_{12} & t_{13} \\
t_{12} & r_{22} & t_{23} \\
t_{13} & t_{23} & r_{33}
\end{array}\right)=\left(\begin{array}{ccc}
r & t & t \\
t & r^{\prime} & t^{\prime} \\
t & t^{\prime} & r^{\prime}
\end{array}\right)
$$


for the junction is obtained, which upon inserting in (3.17) yields by unitarity of the $S$ matrix the single flow equation [DRS04]

$$
\frac{d r^{\prime}}{d \ln \Lambda}=-\alpha\left(r^{\prime}\right)^{2}\left(1+r^{\prime}\right)
$$

The variable $\alpha$ parametrizes the homogeneous interaction in wire (2) and (3), viz. $\alpha=$ $\frac{1-K^{2}}{1+K^{2}}$. Integration from $\ln (1)=0$ to $\ln \left(N_{T} / \pi\right)$ gives 4

$$
r^{\prime}\left(N_{T}\right)=-\frac{1}{\mathrm{~W}\left(\left(1+\frac{1}{r_{0}^{\prime}}\right) e^{\left.-\left(r_{0}^{\prime}+1\right) / r_{0}^{\prime}\left(N_{T} / \pi\right)^{-\alpha}\right)+1}\right.}
$$

with the Lambert $\mathrm{W}$ function $\mathrm{W}$, cf. $\left[\mathrm{CGH}^{+96}\right.$, and initial value $r_{0}^{\prime}=r^{\prime}(0)=$ $2 /\left(2+\left(t_{L}^{\text {coupl }}\right)^{2}\right)$, cf. $(3.18)$. In the regime $N_{L}^{\text {over }} \gg N_{T} \gg N_{B}\left[\pi v_{F} / N_{L}^{\text {over }} \ll T \ll B\right]$, the conductance is obtained by incoherently combining the $\mathrm{S}$ matrices of the junction (4.7) and the open boundary at the end of wire 2 as [Dat95, p. 125 et seq.]

$$
G\left(N_{T}\right) / G_{0}=-4 r^{\prime}\left(N_{T}\right) \frac{1+r^{\prime}\left(N_{T}\right)}{1-r^{\prime}\left(N_{T}\right)}
$$

Fig. 4.12 compares the conductance $G\left(N_{T}\right)$ of a wire with overhanging part $N_{L}^{\text {over }}=$ 1110 and coupling $t_{L}^{\text {coupl }}=0.25$ obtained with the fRG [solid lines] to the one obtained with eq. (4.9) [dash-dotted lines] for different interaction strengths $U$. For $U=0$ the curves coincide well inside the regime $N_{L}^{\text {over }} \gg N_{T} \gg N_{B}\left[\pi v_{F} / N_{L}^{\text {over }} \ll\right.$ $T \ll B]$ in which $(4.9)$ is valid. For $U>0$ the absolute value of the conductance differs, however, the functional form of the curves is identical. To better illustrate this point, the dashed-dotted lines in fig. 4.12 have been offset appropriately. Thus, $G\left(N_{T}\right)$ is a function of the Lambert W function, exhibiting a complicated logarithmic structure. The conductance through the systems of figs. 4.8 and 4.10 in the main text can thus be expected to reflect this logarithmic behavior 5

\section{quantum wire with arbitrarily coupled two-dimensional leads}

Two-dimensional leads (stripes), i. e. $N_{L / R}^{\text {con }}>1$, are connected to the wire as defined in (4.2a) and (4.2b) and as sketched in fig. 4.1. The parameter space grows linearly with the number of coupling sites, e. g. for five sites at each contact, ten couplings $t_{L / R}^{l_{x}, \text { coupl }}$ with $l_{x}=1, \ldots, N_{L / R}^{\text {con }}$ can freely be chosen.

To prevent this proliferation of parameters, the case of equal couplings, i. e. $t_{L / R}^{l_{x}, \text { coupl }}=$ $t_{L / R}^{l_{x}^{\prime}, \text { coupl }}$ for all $l_{x}$ and $l_{x}^{\prime}$, will be focused on in the following subsection. The site index

\footnotetext{
${ }^{4}$ Note that the definition of the thermal length in [DRS04, eq. (11)] differs from the definition 4.3 by a factor of $\pi$.

${ }^{5}$ Note that an addition of the reciprocal conductances of the system considered in this side note and the isolated right end of the systems of figs. 4.8 and 4.10 would not give the correct conductance of these systems as the problem involves three scatterers, cf. [JMSE07.
} 
Figure 4.12: System as in fig. 4.11 with $N_{L}^{\text {over }}=1110$. Conductance as function of the thermal length $N_{T}$ in the regime $N_{L}^{\text {over }} \gg N_{T} \gg N_{B}$ $\left[\pi v_{F} / N_{L}^{\text {over }} \ll T \ll B\right]$ for different interaction strengths $U$, viz. from bottom to top: $U=1.0,0.75,0.5,0.0$. The solid lines were obtained with the fRG, the dash-dotted lines with the poor man's RG, cf. text for details.

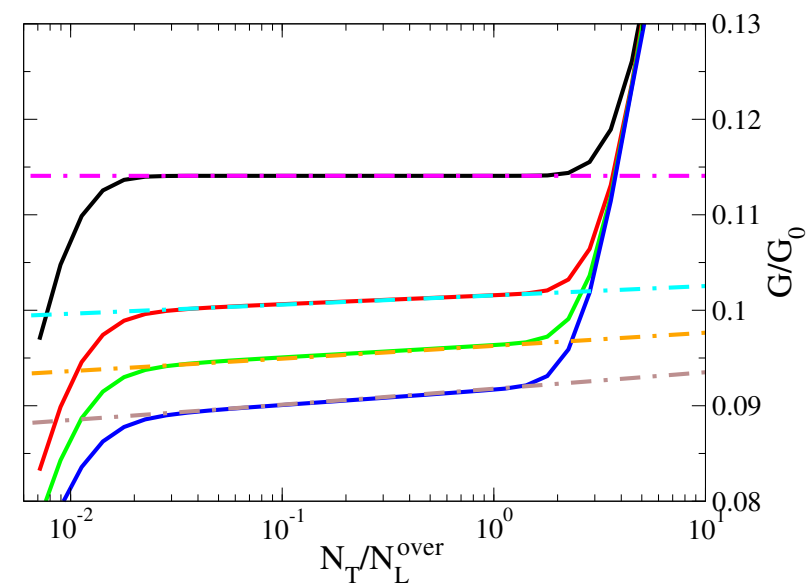

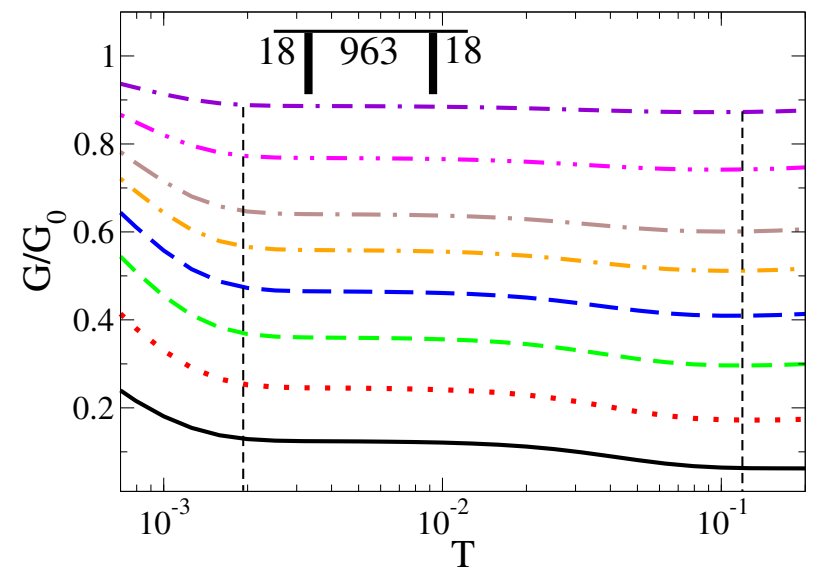

Figure 4.13: Conductance $G$ as function of temperature $T$ of a noninteracting wire coupled to stripe-like leads of different widths into the bulk, viz. $N_{L / R}^{\text {lead }}=0$ and $N_{L}^{\text {over }}=N_{R}^{\text {over }}=$ 18 and $N^{\text {mid }}=963$. The setup is sketched in the inset. All couplings are set equal: $t_{L / R}^{\text {coupl }}=0.25$ and the width of the leads is from bottom to top: $N_{L}^{\text {con }}=N_{R}^{\text {con }}=$ $1,3,5,7,9,11,15,21$. The vertical lines indicate the crossover scales.

$l_{x}$ of the coupling strengths is redundant in that case and will be omitted. Furthermore, the width of the leads is chosen to be equal, i. e. $N_{L}^{\text {con }}=N_{R}^{\text {con }}$. The influence of the relaxation of these restrictions on the basic results presented in the following section will be studied afterwards.

\section{symmetric width and couplings}

In the case of one-dimensional leads, two pronounced even-odd effects were identified. Firstly, the conductance for $T<\pi v_{F} / N$ strongly depended on the overall length $N$ of the wire. This effects persists for two-dimensional lead. The second even-odd effect was traced back to the vanishing local spectral function at the Fermi energy at even sites of the wire. Stripe-like leads couple to at least one odd site of the wire and therefore this effect disappears as the leads get broader.

Fig. 4.13 shows the conductance of a non-interacting wire with $N^{\text {mid }}=963$, overhanging parts of the wire of equal length, $N_{L / R}^{\text {over }}=18$ and leads without overhanging parts, $N_{L / R}^{\text {lead }}=0$ but varying width $N_{L / R}^{\text {con }}$. The vertical lines indicate the temperature scales $\pi v_{F} / N_{L / R}^{\text {over }}$ and $\pi v_{F} / N$, whose importance was recognized and explained for one-dimensional leads in the preceding section. The curves suggest an equal relevance for stripe-like leads as their overall shape remains unaltered if the 
Figure 4.14: As in fig. 4.13 but with length $N^{\text {mid }}=10^{4}+1$ and width $N_{L}^{\text {con }}=N_{R}^{\text {con }}=5$ of the contact regions. Wire coupled in bulk to end of lead, viz. $N_{L / R}^{\text {lead }}=0$ and $N_{L}^{\text {over }}=20$, $N_{R}^{\text {over }}=286$ [red, dash-dotted line], wire coupled at end to bulk of lead, viz. $N_{L / R}^{\text {over }}=0$ and $N_{L}^{\text {lead }}=20, N_{R}^{\text {lead }}=$ 286 [green, dashed line]. For comparison: one-dimensional leads $\left(N_{L / R}^{\text {con }}=\right.$ 1), coupled at ends, viz. $\quad N_{L / R}^{\text {lead }}=0$ and $N_{L / R}^{\text {over }}=0$ [orange, dash-doubledotted line]. Setups are shown in

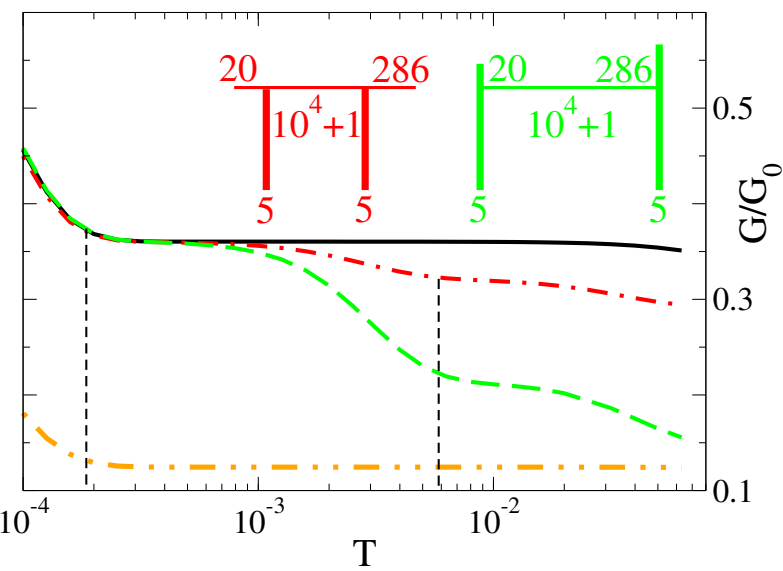
the insets. Vertical lines indicate the crossover scales.

width of the leads is increased. This point will be further discussed below. For large numbers of transverse channels in the leads, $G / G_{0}$ approaches one. This trend can be counteracted by reducing the strength of the couplings between leads and wire.

Fig. 4.14 shows the conductance for systems similar to those in fig. 4.2 , but with two-dimensional leads, $N_{L / R}^{\text {con }}=5$. For comparison, the curve for a wire coupled at its ends $\left(N_{L / R}^{\text {over }}=0\right)$ to the end of each lead $\left(N_{L / R}^{\text {lead }}\right)$ with lead width $N_{L}^{\text {con }}=$ $N_{R}^{\text {con }}=5$ is included. Overall, the conductance in the regime $\pi v_{F} / N \ll T \ll B$ is considerably higher than in the case of one-dimensional leads, cf. fig. 4.2, as expected from the results of fig. 4.13 . At the temperature scales $\pi v_{F} / N, \pi v_{F} / N_{\text {over }}^{L / R}$ and $\pi v_{F} / N_{\text {lead }}^{L / R}$ respectively the conductance crosses over to lower plateaus. This supports the conclusions drawn from fig. 4.13. The complete equivalence of $N_{L}^{\text {over/lead }}$ and $N_{R}^{\text {over/lead }}$ found in the case of one-dimensional leads is lost as the geometric equivalence is no longer present.

In the following, the wire is chosen to be interacting. A typical off-diagonal component $\Sigma_{j, j+1}$ of the self-energy of an interacting wire, coupled in its bulk to stripe-like leads, is shown in fig. 4.15. The parameters chosen are similar to those in fig. 4.6 in order to facilitate comparison to the case of one-dimensional leads. The oscillations emerging from the boundaries are expectedly unaffected by the width of the leads. The contact region itself (of size $N_{L}^{\text {con }}=21$; magnified in lower panel of figure) contains a superposition of oscillations emerging from all couplings sites. The symmetric appearance of the oscillations inside the contact region results from the choice of equal coupling strengths. The oscillations spreading from the outmost left contact site to the left and from the outmost right contact site to the right still fall off with inverse distance up to a scale $j_{T} \sim 1 / T$ beyond which they decay exponentially. Like in the case of one-dimensional leads these oscillations do not induce a powerlaw suppression in the local spectral function within the approximate fRG. These similarities imply that the temperature dependence of $G$ for an interacting wire 


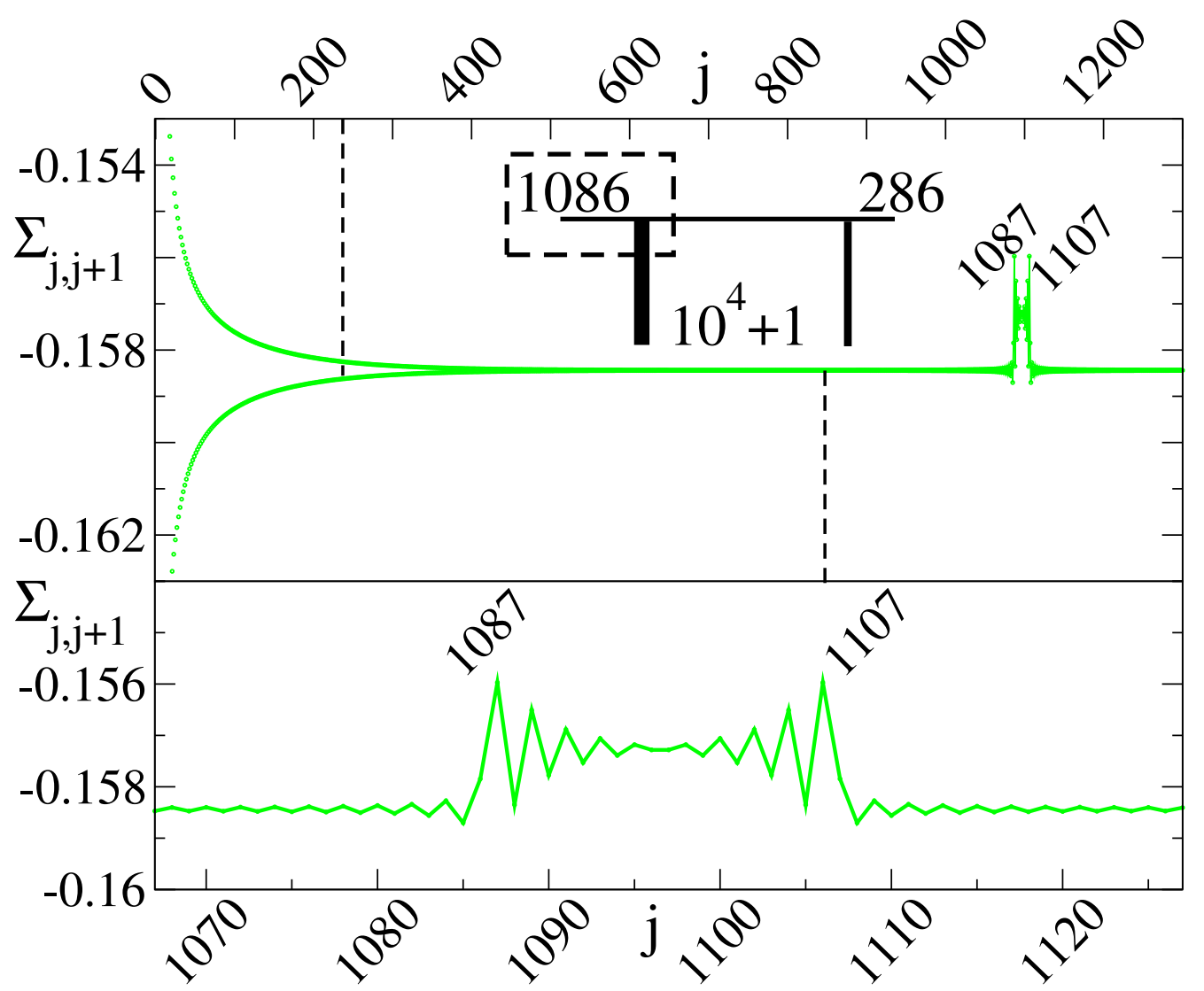

Figure 4.15: Off-diagonal self-energy at the end of the fRG flow for an interacting wire of length $N=10^{4}+1$ with $U=0.5$ and couplings $t_{R}^{\text {coupl }}=t_{L}^{\text {coupl }}=0.25$ at $T=4 \cdot 10^{-3}$. The leads of width $N_{L}^{\text {con }}=21$ and $N_{L}^{\text {con }}=3$ are attached with their end (i. e. $N_{L / R}^{\text {lead }}=0$ ) to the bulk of the wire with $N_{L}^{\text {over }}=1086$ and $N_{R}^{\text {over }}=286$. The part of the self-energy shown in the main plot is indicated by the dashed box in the inset. Long-ranged oscillations emerge from the boundaries and from all coupling sites. The respective scale $j_{T}$ beyond which the oscillations decay exponentially is indicated by the vertical dashed lines. The lower plot is a magnification of the left contact region.

with stripe-like leads can be explained within the reasoning presented in the case of one-dimensional leads.

Fig. 4.16 focuses on the case of an interacting wire, weakly coupled $\left(t_{L}^{\text {con }}=t_{R}^{\text {con }}=\right.$ 0.1 ) at its ends to the end of the leads, i. e. without any overhanging parts. The only energy scales are the inverse overall length $\pi v_{F} / N$ and the bandwidth $B$. In the regime $\pi v_{F} / N \ll T \ll B$, the conductance scales $\sim T^{\alpha_{\text {end }}}$. The closeness of the curves for $N_{L / R}^{\text {con }}=5$ and $N_{L / R}^{\text {con }}=6$ exemplifies the minor importance of the aforementioned even-odd effect. In fig. 4.17 an overhanging part of the leads is added to the system width $N_{L / R}^{\text {con }}=5$, whose conductance curve is shown for comparison [green, dashed line]. Similar to the case of one-dimensional leads, the universally decaying oscillations from the ends of the system generate a power law $G(T) \sim T^{\alpha_{\text {end }}}$ in the three temperature regimes introduced by the overhanging parts. For a wire of length $N=10^{4}+1$ it is difficult to resolve all three temperature 


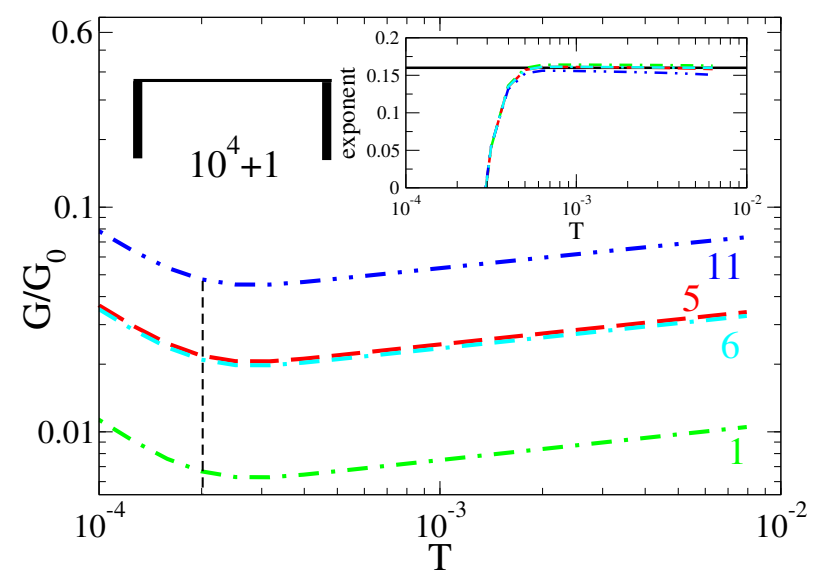

Figure 4.16: Conductance $G$ as function of temperature $T$ of an interacting wire of length $N=10^{4}+1$ with $U=0.5$. The ends of the wire are weakly coupled $\left(t_{L}^{\text {coupl }}=t_{R}^{\text {coupl }}=\right.$ 0.1 ) to leads without any overhanging parts, viz. $N_{L / R}^{\text {lead }}=0$ and $N_{L / R}^{\text {over }}=$ 0 . The width of the leads is varied: $N_{L}^{\text {con }}=N_{R}^{\text {con }}=1,6,5,11$ (from bottom to top, as indicated in the plot). Setup sketched in the left inset. Vertical dashed line indictates the crossover scale. Right inset: Effective exponents, styles and colors as in main plot. Solid horizontal line indicates the fRG approximation for $\alpha_{\text {end }}$, cf. [EMA $\left.{ }^{+} 05\right]$.

Figure 4.17: As in fig. 4.16 but with fixed width of leads, $N_{L}^{\text {con }}=N_{R}^{\text {con }}=5$ and $N_{L}^{\text {lead }}=N_{R}^{\text {lead }}=0$ [green, dashed], $N_{L}^{\text {lead }}=N_{R}^{\text {lead }}=100[$ red, dotted $]$ and $N_{L}^{\text {lead }}=N_{R}^{\text {lead }}=332$ [blue, dashdotted].

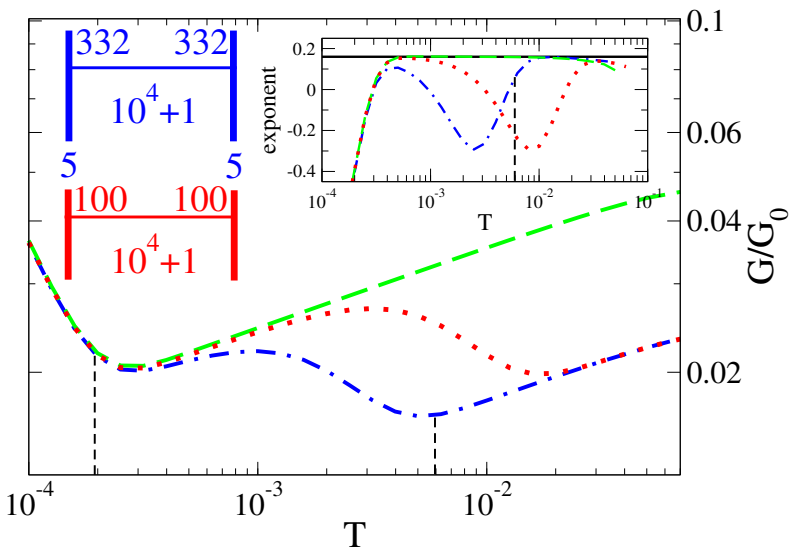

regimes introduced by the overhanging parts due to the broad crossover regions and the upper (bandwidth $B$ ) and lower (inverse overall length $\pi v_{F} / N$ ) bounds of any power-law scaling. Therefor the number of regimes to be resolved was reduced to two by choosing $N_{L}^{\text {lead }}=N_{R}^{\text {lead }}$. Even in this case, the two power laws cannot be resolved for a single set of parameters (compare the blue, dash-dotted line to the red, dotted one in fig. 4.17).

The case of leads coupled at their end to the bulk of the interacting wire is shown in fig. 4.18. As anticipated from the non-interacting case (cf. the discussion of fig. 4.14), the length scales $N_{L}^{\text {over }}$ and $N_{R}^{\text {over }}$ introduce the temperature scales

$$
\begin{array}{r}
\pi v_{F} / N \ll T \ll \pi v_{F} / \max \left(N_{L / R}^{\text {over }}\right) \\
\pi v_{F} / \max \left(N_{L / R}^{\text {over }}\right) \ll T \ll \pi v_{F} / \min \left(N_{L / R}^{\text {over }}\right) \\
\pi v_{F} / \min \left(N_{L / R}^{\text {over }}\right) \ll T \ll B .
\end{array}
$$

In all three regimes, the conductance shows the same behavior as in the case of onedimensional leads. Namely, in the first regime, the oscillations from the boundaries reach well beyond the coupling region, inducing the dependence $G(T) \sim T^{\alpha_{\mathrm{end}}}$. To 


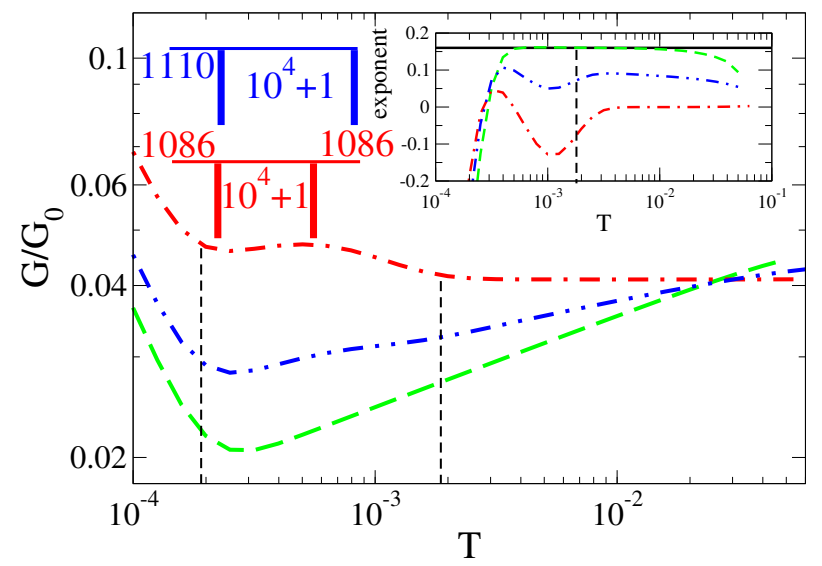

Figure 4.18: As in fig. 4.16 but with fixed width of leads, $N_{L}^{\text {con }}=N_{R}^{\text {con }}=5$ and $N_{L}^{\text {over }}=N_{R}^{\text {over }}=0$ [green, dashed], $N_{L}^{\text {over }}=N_{R}^{\text {over }}=1086[$ red, dashdotted] and $N_{L}^{\text {over }}=1110, N_{R}^{\text {over }}=0$ [blue, dash-double-dotted].

better resolve the conductance curve in the second and third regime, this first regime is chosen such small in fig. 4.18 that the asymptotic value of the exponent is not reached. The second regime is exemplified by the blue, dash-double-dotted curve. At scale $\pi v_{F} / N_{L}^{\text {over }}=\pi v_{F} / N_{R}^{\text {over }}$, the conductance crosses over to a seemingly powerlaw behavior. However, as has been discussed in detail in the case of one-dimensional leads, a complex logarithmic behavior is to be expected and the proper inclusion of bulk Luttinger-liquid behavior is likely to significantly change the curves. In the third regime, the oscillations in the self-energy $\Sigma_{j, j+1}$ from the boundaries are cut off by the exponential falloff (beyond distance $\sim 1 / T$ ) well before reaching the contact region and thus, no power-law behavior can be found within the approximate fRG (red, dash-dotted line in fig. 4.18). However, the conductance is expected to show power-law scaling with the bulk Luttinger-liquid exponent, if computed within a scheme, which properly includes these effects of order $U^{2}$.

The conductance through "mixed" systems with overhanging parts of the leads and the wire again shows an even richer temperature dependence, which can be understood by combining the cases presented above.

\section{asymmetries in width and coupling}

In figs. 4.19 and 4.20 the restriction of equal width of the leads is dropped, i. e. $N_{L}^{\text {con }} \neq N_{R}^{\text {con }}$ is chosen, for similar systems as studied in figs. 4.16 to 4.18 .

Fig. 4.19 focuses on the case of an interacting wire, weakly coupled $\left(t_{L}^{\text {con }}=t_{R}^{\text {con }}=\right.$ 0.1 ) at its ends to leads with overhanging parts $N_{L}^{\text {lead }}=N_{R}^{\text {lead }}=332$. The width of the left lead is significantly higher than the width of the right lead, viz. $N_{L}^{\text {con }}=7$ vs. $N_{R}^{\text {con }}=3$ [orange, dash-double-dotted line]. For comparison the conductance curve of a similar system with coupling regions of equal width, viz. $N_{L}^{\text {con }}=N_{R}^{\text {con }}=5$, is included [blue, dash-dotted line]. Apart from an overall lower conductance in the case of coupling regions of differing length, which results from the smaller number of transverse channels in the right lead $\left(N_{R}^{\text {lead }}=3\right.$ vs. $\left.N_{R}^{\text {lead }}=5\right)$, the form of the curves remains unaltered. The crossover scales are $\pi v_{F} / N$ and $\pi v_{F} / N_{L / R}^{\text {lead }}$ as identified in the preceding subsection. The effective exponent (right inset) shows that the behavior in the two resulting temperature regimes is identical in both cases. 
Figure 4.19: As in fig. 4.17 with overhanging leads $N_{L}^{\text {lead }}=N_{R}^{\text {lead }}=332$ and $N_{L / R}^{\text {over }}=0$, but $N_{L}^{\text {con }}=7$ and $N_{R}^{\text {con }}=3$ [orange, dash-double-dotted] (setup shown in left inset). For comparison: $N_{L}^{\text {lead }}=N_{R}^{\text {lead }}=332$ and symmetric width $N_{L}^{\text {con }}=N_{R}^{\text {con }}=5$ [blue, dash-dotted]; $N_{L / R}^{\text {lead }}=0$ and $N_{L}^{\text {con }}=N_{R}^{\text {con }}=5$ [green, dashed].
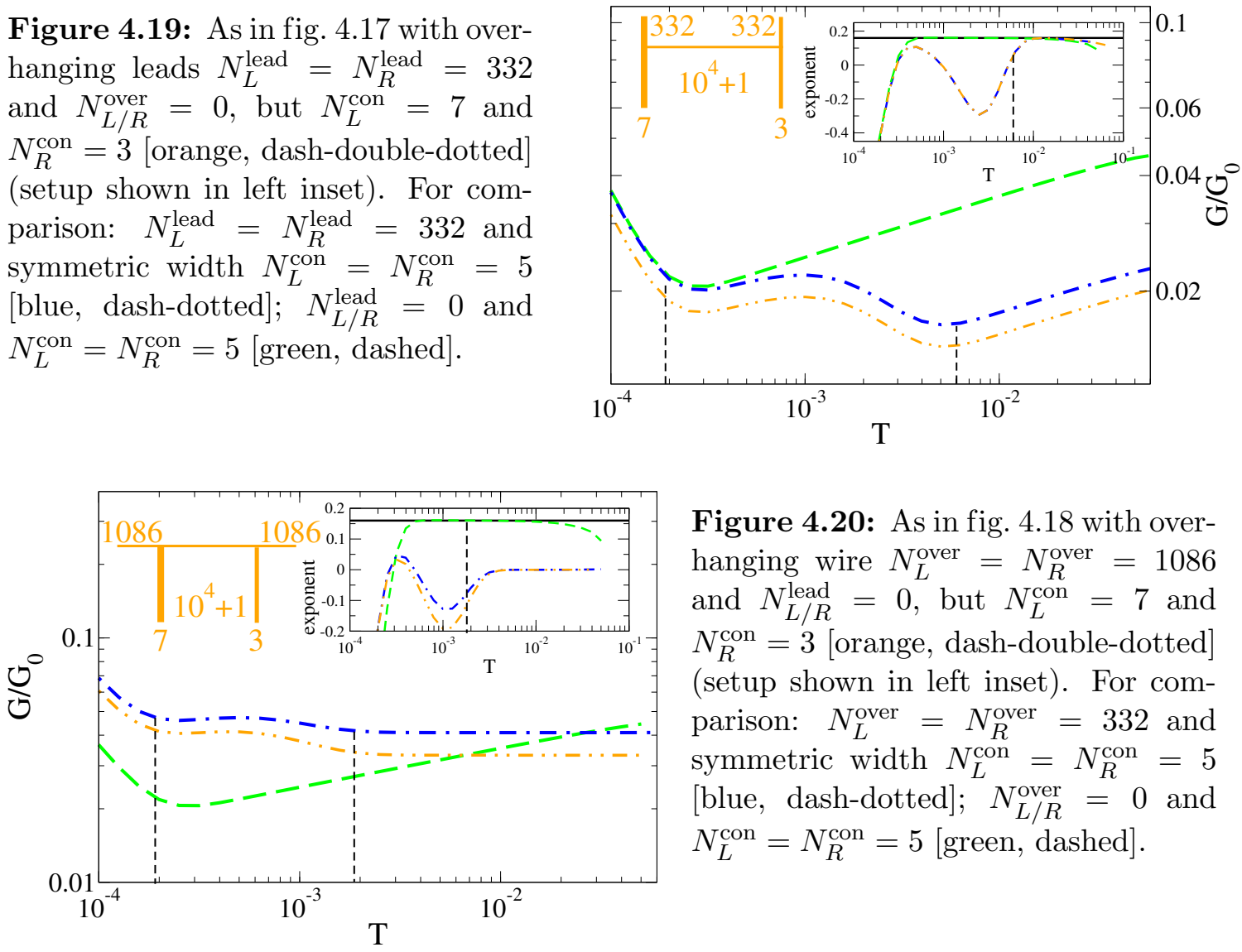

Figure 4.20: As in fig. 4.18 with overhanging wire $N_{L}^{\text {over }}=N_{R}^{\text {over }}=1086$ and $N_{L / R}^{\text {lead }}=0$, but $N_{L}^{\text {con }}=7$ and $N_{R}^{\text {con }}=3$ [orange, dash-double-dotted] (setup shown in left inset). For comparison: $N_{L}^{\text {over }}=N_{R}^{\text {over }}=332$ and symmetric width $N_{L}^{\text {con }}=N_{R}^{\text {con }}=5$ [blue, dash-dotted]; $N_{L / R}^{\text {over }}=0$ and $N_{L}^{\text {con }}=N_{R}^{\text {con }}=5$ [green, dashed].

Fig. 4.20 compares the conductance of an interacting wire coupled in its bulk $\left(N_{L / R}^{\text {over }}=1086\right)$ to the end of the leads $\left(N_{L / R}^{\text {lead }}=0\right)$ with coupling regions of unequal length $\left(N_{L}^{\text {con }}=7\right.$ vs. $\left.N_{R}^{\text {con }}=3\right)$ to a similar wire with coupling regions of equal length $\left(N_{L}^{\text {con }}=N_{R}^{\text {con }}=5\right)$. Similar to the case of the wire coupled at its ends, the crossover scales $\pi v_{F} / N$ and $\pi v_{F} / N_{L / R}^{\text {over }}$ do not change and the effective exponent indicates an identical behavior of both curves. As expected, the lower number of transverse channels in the right lead overall reduces the conductance in the case of coupling regions of unequal length.

The case of locally varying coupling strengths is exemplified in fig. 4.21 and 4.22, for simplicity for identical widths of the coupling regions $\left(N_{L}^{\text {con }}=N_{R}^{\text {con }}\right)$. The coupling strengths $t_{L / R}^{l_{x} \text {,coupl }}$ with $l_{x}=1, \ldots, N_{L / R}^{\text {con }}$ are chosen randomly from the interval $\left[10^{-3}, 0.4\right]$.

A typical off-diagonal component $\Sigma_{j, j+1}$ of the self-energy with similar parameters as in fig. 4.15, but with varying coupling strengths is depicted in fig. 4.21. The oscillations emerging from the boundaries are expectedly unaffected, therefore only the zoomed-in coupling region (of size $N_{L}^{\text {con }}=21$ ) is shown. As anticipated in the description of $\Sigma_{j, j+1}$ for equal coupling strengths, the symmetric appearance of the superposition of the oscillations emerging from all coupling sites is lost and the randomness of the couplings is reflected. However, the oscillations spreading from the outmost left contact site to the left and from the outmost right contact site to 


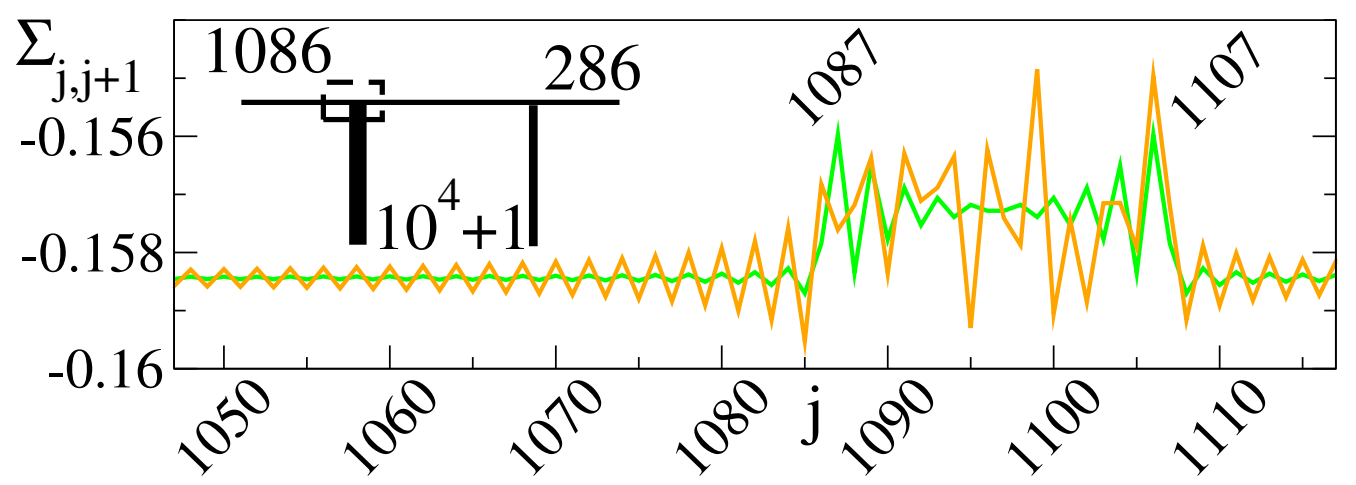

Figure 4.21: Off-diagonal self-energy at the end of the fRG flow for an interacting wire similar to fig. 4.15, but with coupling strengths chosen randomly from the interval $\left[10^{-3}, 0.4\right]$, [orange]. Green line as in fig. 4.15 for comparison. The part of the self-energy shown is indicated by the dashed box in the inset.

Figure 4.22: As in fig. 4.18 and fig. 4.17, respectively, viz. $N_{L / R}^{\text {over }}=$ $0, N_{L / R}^{\text {lead }}=0$ [orange, dash-dotted], $N_{L / R}^{\text {over }}=0, N_{L}^{\text {lead }}=N_{R}^{\text {lead }}=332[$ violet, dash-double-dotted], $N_{L}^{\text {over }}=N_{R}^{\text {over }}=$ 1086, $N_{L / R}^{\text {lead }}=0$ [cyan, dotted], each with $N_{L}^{\text {con }}=N_{R}^{\text {con }}=5$ but with coupling strengths chosen randomly from the interval $\left[10^{-3}, 0.4\right]$. For comparison: $N_{L / R}^{\text {over }}=0, N_{L / R}^{\text {lead }}=0$ and $N_{L}^{\text {con }}=$ $N_{R}^{\text {con }}=5$ [green, dashed] with identical coupling strengths $\left(t_{L}^{\text {coupl }}=t_{R}^{\text {coupl }}=\right.$

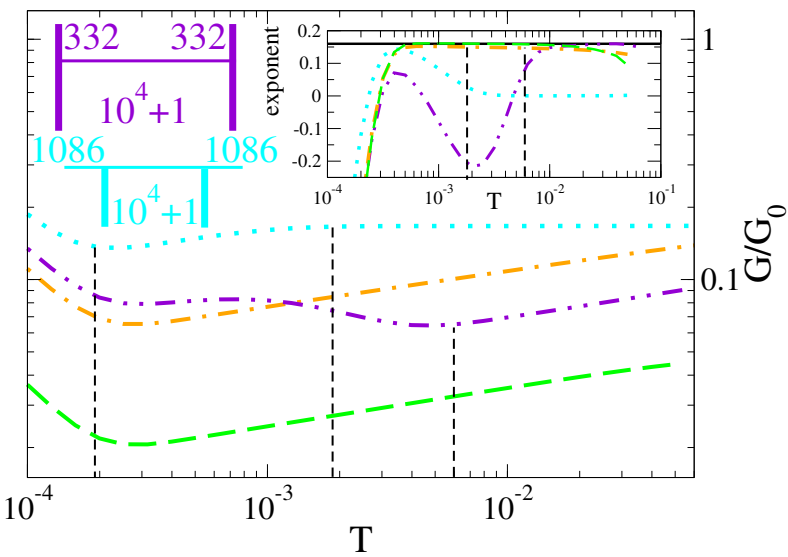
$0.1)$.

the right still fall off with inverse distance up to a scale $j_{T} \sim 1 / T$ beyond which they decay exponentially and essentially remain unaffected by the randomness inside the coupling region. The somewhat bigger amplitude of these oscillations stems from the fact, that the coupling strengths are partly higher than 0.1 , which was exemplarily chosen in the case of equal couplings, cf. fig. 4.15. This similar behavior of the oscillations propagating to the left and right implies a temperature dependence of $G$, which is similar to the case of equal couplings.

Fig. 4.22 shows the conductance through an interacting wire $(U=0.5)$ with coupling regions of equal widths $\left(N_{L}^{\text {con }}=N_{R}^{\text {con }}=5\right)$ but with randomly chosen coupling strengths. The geometries are chosen as in fig. 4.18 and fig. 4.17 and the same random set of coupling strength is used in all curves ${ }^{6}$ in order to facilitate comparison.

\footnotetext{
${ }^{6}$ Namely: $t_{L}^{1 \ldots 5, \text { coupl }}=\{0.069485,0.226153,0.307347,0.171172,0.065679\}$ and $t_{R}^{1 \ldots 5, \text { coupl }}=\{0.196028,0.277192,0.227531,0.358452,0.064737\}$.
} 
For all three systems, the overall conductance is somewhat higher than in the case of equal couplings $\left(t_{L / R}^{\text {coupl }}=0.1\right.$, cf. figs. 4.17 and 4.18 due to the partly higher coupling strengths. The energy scales induced by the geometry (cf. previous sections) remain unaffected by the randomness of the couplings, as can be inferred from the effective exponent in the inset (the vertical lines indicate the crossover scales). Explicitely, in the case of leads coupled at their ends to the ends of the wire $\left(N_{L / R}^{\text {over }}=N_{L / R}^{\text {lead }}=0\right)$ [orange, dash-dotted line], the effective exponent (shown in the right inset) shows a clear plateau of height $\alpha_{\text {end }}$ in the regime $\pi v_{F} / N \ll$ $T \ll B$ (the conductance curve for equal couplings $t_{L / R}^{\text {coupl }}=0.1$ is shown in the main plot and inset for comparison [green, dashed line]). The small deviation from the curve of equal coupling stems from the fact that some couplings are considerably higher than 0.1, therefore moving the asymptotic regime to bigger system sizes, cf. e. g. $\left[\mathrm{EMA}^{+}\right.$05, $\mathrm{AEM}^{+}$04]. The conductance through the system with overhanging parts of the leads $\left(N_{L / R}^{\text {over }}=0\right.$ and $\left.N_{L}^{\text {lead }}=N_{R}^{\text {lead }}=332\right)$ [violet, dash-double-dotted] shows two plateaus with exponent $\alpha_{\text {end }}$ in the regimes $\pi v_{F} / N \ll T \ll \pi v_{F} / N_{L / R}^{\text {lead }}$ and $\pi v_{F} / N_{L / R}^{\text {lead }} \ll T \ll B$. Equally, the conductance curve for the geometry with overhanging parts of the wire $\left(N_{L}^{\text {lead }}=N_{R}^{\text {lead }}=1086\right.$ and $\left.N_{L / R}^{\text {lead }}=0\right)$ [cyan, dotted line] crosses over at scale $\pi v_{F} / N_{L / R}^{\text {over }}$ from a regime with power-law behavior to a regime in which no scaling behavior can be found within the approximate fRG. Noteably, the conductance is not lowered before reaching the plateau, but stays at the value reached at the end of the scaling regime. This is in accordance with the observation that the crossover for the wire coupled in its bulk becomes less pronounced for increasing width of the leads, cf. figs. 4.13 and 4.14 .

In summary, neither the relaxation of the constriction to symmetric coupling regions nor to equal coupling strengths alters the basic behavior of the conductance as function of temperature. In particular, the crossover scales are unaffected and universally present.

\section{side note: the role of spin}

The results for the conductance of the quantum wire can directly be generalized to spin- $1 / 2$ fermions by appropriately adapting the Luttinger-liquid parameter $K$ and the exponent $\alpha$ in the scaling laws, cf. section 2.1, if the interaction is long-ranged (that is the $g_{1, \perp}$-type interactions, cf. section 2.1 can be neglected as the interaction is short-ranged in $k$-space and thus momentum transfer of $\approx 2 k_{F}$ can be neglected) and the system is spin rotationally invariant. For single wall carbon nanotubes this is generally assumed to be the case [KBF97, EG97]. In presence of a term $g_{1, \perp}>0$, the asymptotic low-energy sector is usually reached via a complex crossover-regime, e. g. $\mathrm{MMS}^{+} 00$, YGM94, $\mathrm{AEM}^{+} 06 \mathrm{~b}$. This significantly increases the system sizes needed to reach the asymptotic behavior $\left[\mathrm{AEM}^{+} 06 \mathrm{~b}\right]$. 



\section{quantum-dot structures with Luttinger-liquid leads}

Parts of the results presented in this chapter have been published in [WMS07a].

\section{summary}

Section 5.1 explains, how the technique to include two-dimensional leads in the approximate fRG, cf. chapter 4 , can be used to study arbitrary quantum-dot structures coupled to Luttinger-liquid leads.

The conductance and charging of a single dot with two Luttinger-liquid leads, fig. 5.1 (a), is investigated in section 5.2. In particular, several characteristic power laws in the charging are identified using perturbation theory and fRG. With these results, the experimental setup presented in the introduction is further specified. Other single-dot geometries with Luttinger-liquid leads, fig. 5.1 (b)/(c), are discussed in a side note.

In section 5.3, the implementation of the fRG scheme from section 5.1 is exemplified for a system of two quantum dots, coupled in parallel to Luttinger-liquid leads, fig. 5.1 (d). The conductance and the local spectral functions are studied. Thereby, the connection to the problem of a double barrier in a Luttinger liquid is thoroughly investigated and the relation to the recent study [RS04] is discussed.

In a final side note, another example for the implementation of the fRG scheme is given and used to study the impact of dot-lead interactions on the conductance through a Luttinger liquid wire with side-coupled quantum dot. 


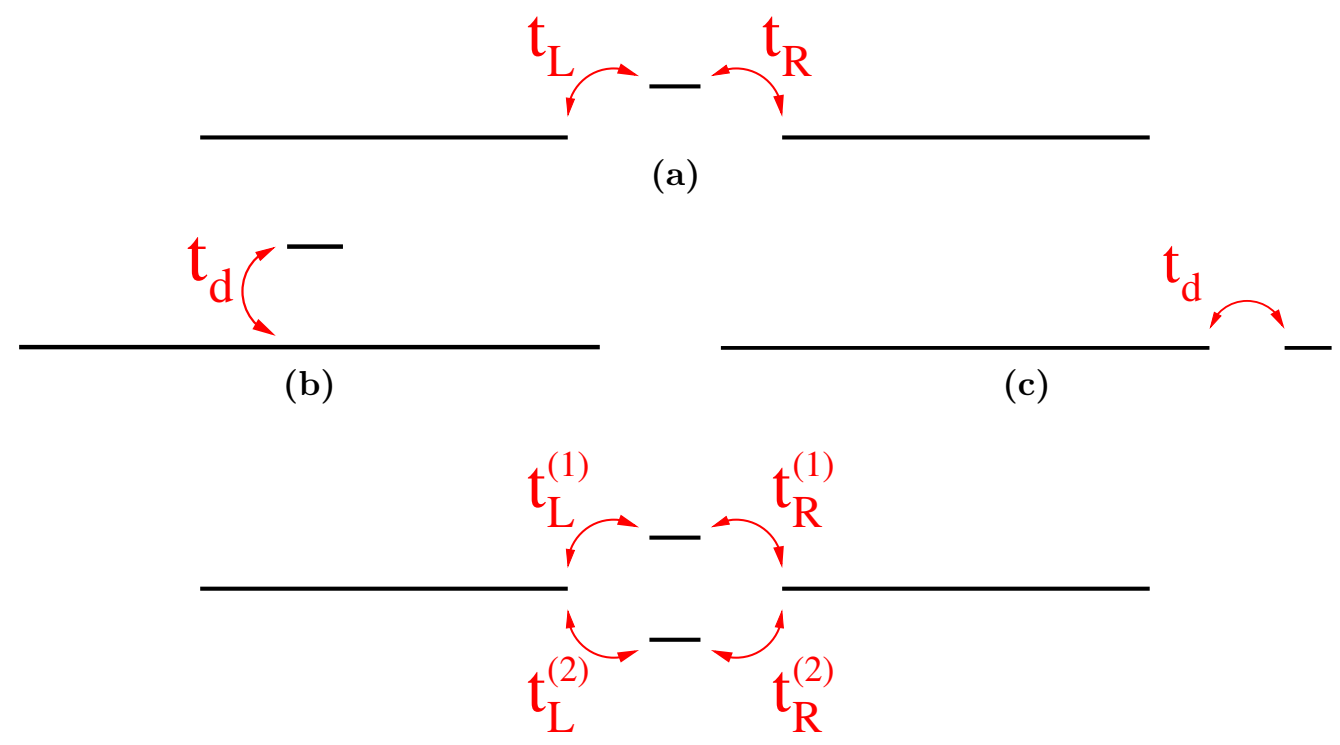

(d)

Figure 5.1: (a) single dot: single level coupled via hopping terms $t_{L}, t_{R}$ to the end of semi-infinite Luttinger liquids. (b) side-coupled dot: single level coupled via a hopping term $t_{d}$ into the bulk of an infinite Luttinger liquid. (c) end-coupled dot: single level coupled via a hopping term $t_{d}$ to the end of a semi-infinite Luttinger liquid. (d) parallel double dot: two single levels, coupled in parallel to the end of semi-infinite Luttinger liquids.

\section{dot structures coupled to Luttinger-liquid leads within the approximate fRG}

Quantum-dot structures coupled to Luttinger-liquid leads can be described using the lattice model introduced in section 2.2. The general setup is depicted in fig. 5.2 . The two Luttinger-liquid leads $(L / R)$ of length $N_{L}$ respectively $N_{R}$ are modelled by the Hamiltonian $H_{L / R}^{\text {wire }}$ given by eq. (2.5) with the lengths $N_{L}, N_{R}$ inserted appropriately. At the end (sites 1) each is coupled smoothly (cf. the side note: the stub in poor man's $R G$ on page 40 to a one-dimensional non-interacting hopping chain, eq. (2.8). The hopping in the non-interacting leads is set to equal the hopping $t$ in the wires, which is used as energy scale, $t=1$. The dot structure is described by some Hamiltonian $h^{\text {dot }}$, which includes the energy levels of the dots, the interdot hopping(s) and the coupling(s) of the $\operatorname{dot}(\mathrm{s})$ to the sites $N_{L}$ and $N_{R}$ of the leads, such that the complete Hamiltonian reads

$$
H=H_{L}^{\text {wire }}+H_{R}^{\text {wire }}+h_{L}^{\text {coupl }}+h_{R}^{\text {coupl }}+h_{L}^{1 \mathrm{~d}-\text { lead }}+h_{R}^{1 \mathrm{~d}-\text { lead }}+h^{\text {dot }}\left(\left\{d^{\dagger}, d\right\}\right)
$$

with $\left\{d^{\dagger}, d\right\}$ representing the set of dot operators. The electrons on the dots are assumed not to interact with the electrons in the leads. For large $N_{L / R}$ this is a microscopic model for the specific dot structure coupled to two semi-infinite Luttingerliquid leads. 


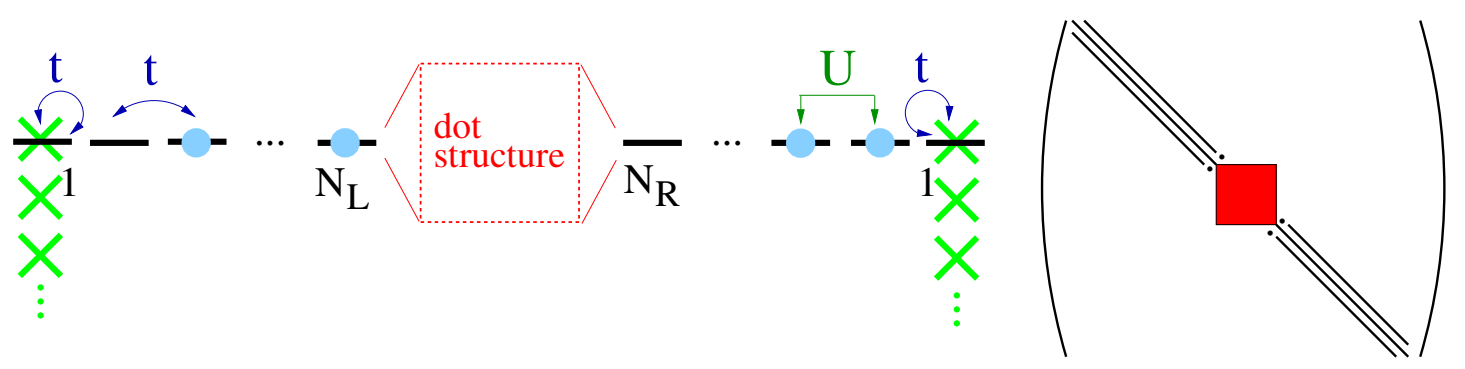

Figure 5.2: Left plot: Dot structure [red box], coupled to two finite one-dimensional hopping chains of length $N_{L / R}$ with nearest-neighbour hopping $t$ and nearest-neighbour interaction $U$. Each chain is coupled smoothly (cf. text) to a semi-infinite, one-dimensional chain [green crosses]. The asymptotic low-energy behavior (reached for large $N_{L / R}$ ) is similar to the physics of an identical dot structure coupled to two semi-infinite Luttingerliquid leads. Right plot: structure of the inverse Green functions during the fRG flow.

To implement this model in the approximate fRG, all energy levels, i. e. those of the leads and those of the dot structure are regarded as a single chain. The dot structure is established by connecting the appropriate energy levels by nearestneighbour and longer range hoppings, such that the wanted coupling geometry is obtained. As the charge on the dots is assumed neither to interact with charge on other dots nor with charge in the leads, the dot levels, the interdot- and dotlead hoppings are not renormalized during the fRG flow ${ }^{1}$ Two cases have to be discriminated.

Quantum dots, modelled as single energy levels and coupled in series only involve nearest-neighbour couplings and therefore, all matrices needed in the flow equations of the approximate fRG (cf. section 3.1.2) are tridiagonal, such that the algorithm from appendix C.1 can be used. A detailed fRG study of this situation can be found in $\left[\mathrm{EMA}^{+} 05\right]$.

Quantum dots, modelled as single energy levels but (partly) coupled in parallel, involve longer range hoppings (at least next-nearest-neighbour hoppings), such that the matrices needed in the flow equations of the approximate fRG have the structure schematically shown on the right-hand side of fig. 5.2. The dot structure introduces a full matrix (red box) embedded between the two tridiagonal parts, describing the leads. This can be treated using the scheme developed in chapter 4 for a quantum wire coupled to two-dimensional leads. A detailed example is presented in section 5.3 .

A case study of interaction between the electrons on the dots and in the leads is presented in the side note: dot-lead interaction within the approximate $f R G$.

\footnotetext{
${ }^{1}$ Therefor, eqs. (3.15) and (3.16) can trivially be adapted.
} 


\section{single dot with two Luttinger-liquid leads (a)}

A single level (quantum dot) is coupled to the ends of two semi-infinite Luttinger liquids by two hopping terms $t_{L}, t_{R}$ respectively, cf. fig. 5.1 (a). The two Luttinger liquids are chosen to be identical and their chemical potential $\mu$ is set to zero (halffilling). The energy of the level relative to this potential is given by the gate voltage $V_{g}$, temperature $T=0$ is assumed.

In the following subsections, results on the conductance $G$ are reviewed and the charging $n_{j}$ of the dot is studied as function of the gate voltage $V_{g}$ within perturbation theory in the dot-lead couplings and, complementary, with the approximate fRG on a microscopic lattice model, cf. section 5.1 .

\section{conductance $\quad 5.2 .1$}

Concerning the linear conductance $G$, this system amounts in a double barrier placed in a Luttinger liquid. This problem was intensively studied within bosonic field theories using various approximate-analytical, cf. [CW93, FN93, Saf97, Fur98, TGC ${ }^{+}$02, KCSK02, TEG05, RG, cf. [KF92c, KF92a, KF92b] and numerical, cf. [HE04], methods. An adaption of the poor man's RG from chapter 3 was used in [PG03, NG03] and a detailed study using the approximate fRG from section 3.1 on a microscopic model can be found in $\left[\mathrm{EMA}^{+} 05\right]$. The conductance as function of temperature was found to follow different power laws with exponents depending on $K$ in different temperature regimes, depending on the choice of the dot parameters.

Specifically, for a fixed gate voltage $V_{g} \neq 0$ (at $V_{g}=0$ the electrons tunnel resonantly) and $T \gg \pi v_{F} / N$ ( $N$ is infinite in the case of two semi-infinite Luttingerliquid leads), $G \sim T^{2 \alpha_{\text {end }}}$ holds for all $K<1$. On resonance, i. e. at $V_{g}=0$, and for symmetric dot-lead coupling (barriers) $t_{L}^{2}=t_{R}^{2}$, the conductance stays pinned to $G_{0}$ showing a resonance peak whose width scales like $T^{1-K}$. For asymmetric couplings, the conductace vanishes even at $V_{g}=0$, cf. the studies cited above, e. g. [EMA $\left.{ }^{+} 05\right]$.

\section{charging $\quad 5.2 .2$}

In the case of non-interacting leads, the charging $n_{0}\left(V_{g}\right)$ of the dot is given by [BF04, p. 148]

$$
n_{0}\left(V_{g}\right)=\frac{1}{2}-\frac{1}{\pi} \arctan \left(\frac{V_{g}}{\Gamma / 2}\right)
$$

with $\Gamma:=t_{L}^{2}+t_{R}^{2}$. Thus, $n_{0}\left(V_{g} \rightarrow 0\right)$ is always linear to leading order in $V_{g}$ and the characteristic width $W$ around $V_{g}=0$ over which the charge on the dot changes from unity to zero is propotional to $\Gamma$. 


\section{perturbation theory}

All calculational details have been moved to appendix F to condense the presentation.

By definition of the self-energy $\Sigma^{\operatorname{dot}}(z)$ (e. g. [NO98, p. 113]), the full Green function $\mathcal{G}^{\text {dot }}$ on the dot is given by

$$
\mathcal{G}^{\operatorname{dot}}(z)=\left(\left[\mathcal{G}_{0}^{\operatorname{dot}}\right]^{-1}(z)-\Sigma^{\operatorname{dot}}(z)\right)^{-1},
$$

with $\mathcal{G}_{0}^{\operatorname{dot}}(z)=1 /\left(z-V_{g}\right)$ being the Green function of the isolated $\operatorname{dot}\left(t_{L}=t_{R}=0\right)$. In lowest order perturbation theory in $\Gamma$, the self-energy $\Sigma^{\operatorname{dot}}(z)=\Gamma \mathcal{G}^{\text {end }}(z)$ with $\mathcal{G}^{\text {end }}(z)$ being the single-particle Green function of the disconnected semi-infinite Luttinger-liquid at the boundary $\left.\right|^{2}$ This perturbative expansion of the self-energy is superior to the perturbation theory in the Green function, used e. g. to study the charging of a dot side-coupled to a chiral Luttinger liquid in FM02 as it preserves the correct pole structure of the Green function, cf. e. g. [GV05, p. 297 et seq.].

The low-energy behavior of the imaginary part of the retarded Green function $\mathcal{G}_{\text {ret }}^{\text {end }}(\omega)$ (the local spectral function, cf. (3.20) ) is given by

$$
\rho^{\text {end }}=-\frac{\omega_{\mathrm{c}}}{\pi} \mathfrak{I m} \mathcal{G}_{\text {ret }}^{\text {end }}(\omega)=\frac{\Theta\left(\omega_{\mathrm{c}}-|\omega|\right)}{2 K}\left|\frac{\omega}{\omega_{\mathrm{c}}}\right|^{1 / K-1},
$$

cf. section 2.1, whereat a finite support $\left[-\omega_{\mathrm{c}}, \omega_{\mathrm{c}}\right]$ is imposed by means of the step function $\Theta$. The real part can be computed by a Hilbert transformation (KramersKronig relation [Mah00, p. 360]). Its leading behavior for $\left|\omega / \omega_{\mathrm{c}}\right| \ll 1$ is given by

$$
\omega_{c} \mathfrak{R e} \mathcal{G}_{\text {ret }}^{\text {end }}(\omega) \sim \begin{cases}-\operatorname{sgn}(\omega)\left|\omega / \omega_{c}\right|^{1 / K-1} & \text { for } 1 / 2<K<1 \\ \omega / \omega_{c} \ln \left|\omega / \omega_{c}\right| & \text { for } K=1 / 2 \\ -\omega / \omega_{c} & \text { for } K<1 / 2 .\end{cases}
$$

Inserting the perturbative self-energy into definition $(3.20)$, the local spectral function $\rho^{\text {dot }}$ on the dot follows as

$$
\omega_{c} \rho^{\mathrm{dot}}=\frac{\Gamma \rho^{\mathrm{end}}(\omega)}{\left(\omega-V_{g}-\Gamma \mathfrak{R e} \mathcal{G}_{\mathrm{ret}}^{\text {end }}(\omega)\right)^{2}+\left(\Gamma \rho^{\mathrm{end}}(\omega)\right)^{2}} .
$$

The charge $n^{\text {dot }}$ on the dot follows as (cf. (3.22)

$$
n^{\operatorname{dot}}\left(V_{g}\right)=\int_{-\omega_{c}}^{\mu=0} d \omega \rho^{\operatorname{dot}} .
$$

\footnotetext{
${ }^{2}$ In GWB09 the validity of this perturbation theory in the coupling $\Gamma$ was doubted by unsuccessfully applying it to a different system, viz. a single level coupled to the end of a single Luttinger-liquid lead. However, this obviously does not disprove the validity in the system studied here, just as a successful application would not have proved it. Furthermore the perturbation theory was doubted with reference to the RG analysis of the double-barrier problem in KF92b], which identified the coupling to be relevant (in the RG sense) on resonance, cf. [KF92b, p. 15244]. However, this argument fails as all results presented below describe the off resonance regime (that is the behavior of $n\left(V_{g} \neq 0\right)$ ). On resonance $n\left(V_{g}=0\right)=1 / 2$ is readily obtained in the continuous regime, cf. text.
} 


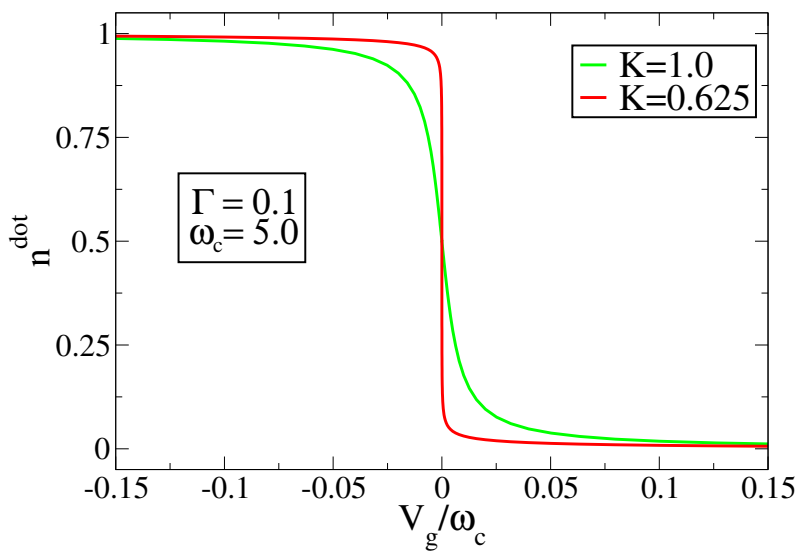

Figure 5.3: Charge $n^{\text {dot }}$ on the dot as function of gate voltage $V_{g}$ for $K=1.0$ [green] and $K=0.625$ [red], $\Gamma=0.1$ and $\omega_{c}=5.0$.

Note that the particle-hole symmetry $(\mu=0)$ implies $n^{\operatorname{dot}}\left(V_{g}\right)=1-n^{\operatorname{dot}}\left(-V_{g}\right)$, which allows to focus on $V_{g} \geq 0$.

For $K \in(1 / 2,1], n^{\operatorname{dot}}\left(V_{g}\right)$ is a continuous function with $n^{\operatorname{dot}}\left(V_{g}=0\right)=1 / 2$, cf. fig. 5.3 for exemplary curves. It contains regular terms of odd order in $V_{g}$, viz. terms $\sim V_{g}^{2 l+1}$ with $l \in \mathbb{N}_{0}$, along with anomalous terms with exponents containing the parameter $K$. The leading of these is $\sim V_{g}^{(2 K-1) /(1-K)}$. Depending on $K$ either the linear $(l=0)$ regular term or the leading anomalous term dominates $n^{\operatorname{dot}}\left(V_{g}\right)$. For $K=2 / 3,(2 K-1) /(1-K)=1$ and logarithmic corrections appear. Explicitely, the leading $V_{g}$ dependence is given by

$$
\frac{1}{2}-n^{\operatorname{dot}}\left(V_{g}\right) \sim \begin{cases}V_{g} / \omega_{c} & \text { for } 2 / 3<K \leq 1 \\ V_{g} / \omega_{c}\left|\ln \left(V_{g} / \omega_{c}\right)\right| & \text { for } K=2 / 3 \\ \left(V_{g} / \omega_{c}\right)^{(2 K-1) /(1-K)} & \text { for } 1 / 2<K<2 / 3,\end{cases}
$$

At $K=1 / 2, n^{\operatorname{dot}}\left(V_{g}\right)$ still is continuous and approaches $1 / 2$ with corrections $\sim$ $1 /\left|\ln \left(V_{g} / \omega_{c}\right)\right|$ for $V_{g} \searrow 0$.

For $K \in(0,1 / 2)$ and for small $\Gamma, n^{\operatorname{dot}}\left(V_{g}\right)$ is discontinuous and shows a jump $\Delta$ at $V_{g}=0$, i. e. $n^{\operatorname{dot}}\left(V_{g} \searrow 0\right) \neq n^{\operatorname{dot}}\left(V_{g} \nearrow 0\right)$, cf. fig. 5.4 for an exemplary curve. In this regime, the results obtained here with the perturbative $\Sigma^{\text {dot }}$ are identical to those of [FM02]. Absorbing the real part of $\mathcal{G}_{\text {ret }}^{\text {end }}(\omega)$, which is linear for $K<1 / 2$, cf. (5.4), in the first term of the denominator of (5.5) and neglecting all but the leading order in $\Gamma$ gives

$$
n\left(V_{g}\right) \sim \Gamma \int_{0}^{\omega_{c}} \frac{\omega^{1 / K-1}}{\left(\omega+V_{g}\right)^{2}},
$$

which is identical to [FM02, eq. (4)]. The jump $\Delta$ at $V_{g}=0$ is given by $\Delta=$ $1-2 n^{\operatorname{dot}}\left(V_{g} \searrow 0\right)$ with $n^{\operatorname{dot}}\left(V_{g} \searrow 0\right)=\frac{\Gamma}{\omega_{c}^{2}(2-4 K)}$. This object is nonuniversal as it depends on the cutoff $\omega_{c}$. For $K \rightarrow 1 / 2$ this expression only holds for sufficiently small $\Gamma$. The corrections to $n$ for finite $V_{g}$ and small $\Gamma$ are given by

$$
n^{\operatorname{dot}}\left(V_{g} \searrow 0\right)-n^{\operatorname{dot}}\left(V_{g}\right) \sim \begin{cases}\left(V_{g} / \omega_{c}\right)^{1 / K-2} & \text { for } 1 / 3<K<1 / 2 \\ V_{g} / \omega_{c}\left|\ln \left(V_{g} / \omega_{c}\right)\right| & \text { for } K=1 / 3 \\ V_{g} / \omega_{c} & \text { for } 0<K<1 / 3 .\end{cases}
$$


Figure 5.4: Charge $n^{\text {dot }}$ on the dot as function of gate voltage $V_{g}$ for $K=$ $0.42, \Gamma=0.01$ and $\omega_{c}=5.0$. The curve exemplifies the jump $\Delta$, which is characteristic for the regime $K<\frac{1}{2}$, cf. text.

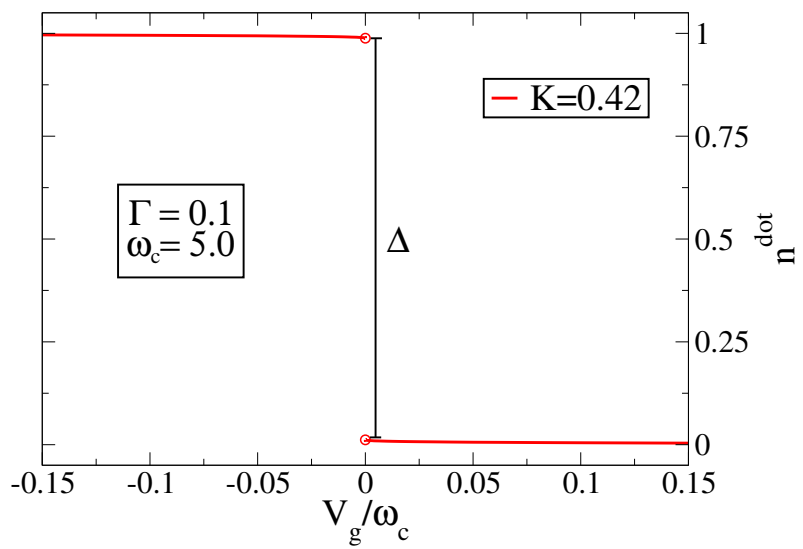

In the case of a continuous $n^{\operatorname{dot}}\left(V_{g}\right)$, i. e. for $K \in(1 / 2,1]$, there exists a characteristic width $W$ around $V_{g}=0$ over which the charge on the dot changes from unity to zero. This width can e. g. be defined ${ }^{3}$ by $W=2 V_{g}^{\text {ch }}$ with $n^{\text {dot }}\left(V_{g}^{\text {ch }}\right)=1 / 4$ and is a function of $\Gamma$. For $\Gamma \rightarrow 0$, the width exhibits power-law scaling following

$$
\frac{W(\Gamma)}{\omega_{c}} \sim\left(\frac{\Gamma}{\omega_{c}^{2}}\right)^{K /(2 K-1)}
$$

over the entire regime $1 / 2<K \leq 1$. This appearance of an anomalous exponent in $W$ seems to contradict the linear $V_{g}$ dependence of $1 / 2-n\left(V_{g}\right)$ for $2 / 3<K \leq 1$. However, both results are consistent as the regime over which $n\left(V_{g}\right)$ goes linearly through $1 / 2$ around $V_{g}=0$ shrinks with decreasing $\Gamma$ and decreasing $K$. This behavior of the width was also reproduced in the case of a dot coupled to the end of a single Luttinger-liquid lead (cf. fig. 5.1 (c)) [GWB09, eq. (9)].

\section{microscopic modelling and fRG}

Complementary to the results obtained with the perturbative approach, the charging of the dot can be calculated within the fRG approach presented in section 5.1. This is non-perturbative in $\Gamma$, but approximate in the interaction, in contrast to the perturbation theory in $\Gamma$ presented above. The single-dot system, cf. fig. 5.1, is trivially obtained by replacing the red box in fig. 5.2 by a single level plus couplings to the left and right $]^{4}$ thus choosing $h^{\text {dot }}=V_{g} d^{\dagger} d-\sum_{a=L, R} t_{a} d^{\dagger} c_{N_{a}}+$ H.c. Note that at half-filling, the leads are only Luttinger liquids for $U<2(K>1 / 2)$, cf. section 2.2 .

With the effective one-particle Hamiltonian obtained at the end of the fRG flow, cf. section 3.1.2, the local spectral function $\rho^{\text {dot }}$ on the dot can be computed using 3.20). Fig. 5.5 shows $\rho^{\operatorname{dot}}(\omega)$ for $U=0.5$, symmetric barriers $t_{L}=t_{R}=\sqrt{0.1}, V_{g}=$

\footnotetext{
${ }^{3}$ This definition is chosen in order to simplify the calculation presented in appendix F In the subsequent calculations on a microscopic model a different definition, yet equivalent concerning the scaling properties, is chosen.

${ }^{4}$ In this simple case, the system can obviously be studied within the formalism for dots coupled in series $\left[\mathrm{EMA}^{+} 05\right]$.
} 
Figure 5.5: Local spectral function on the dot as function of $\omega$ from fRG calculation with $U=0.5, t_{L}=t_{R}=$ $\sqrt{0.1}, V_{g}=1$ and length $N=10^{2}$ [red, dash-dotted], $N=10^{4}$ [green, dashed] and $N=10^{5}$ [black, solid]. Note the logarithmic scale of the $y$ axis. Lower inset: Magnification of $\rho^{\text {dot }}$ around $\omega=0$. Upper inset: $n^{\operatorname{dot}}\left(V_{g}\right)$ for the same parameters as in main plot and $N=10^{5}$.
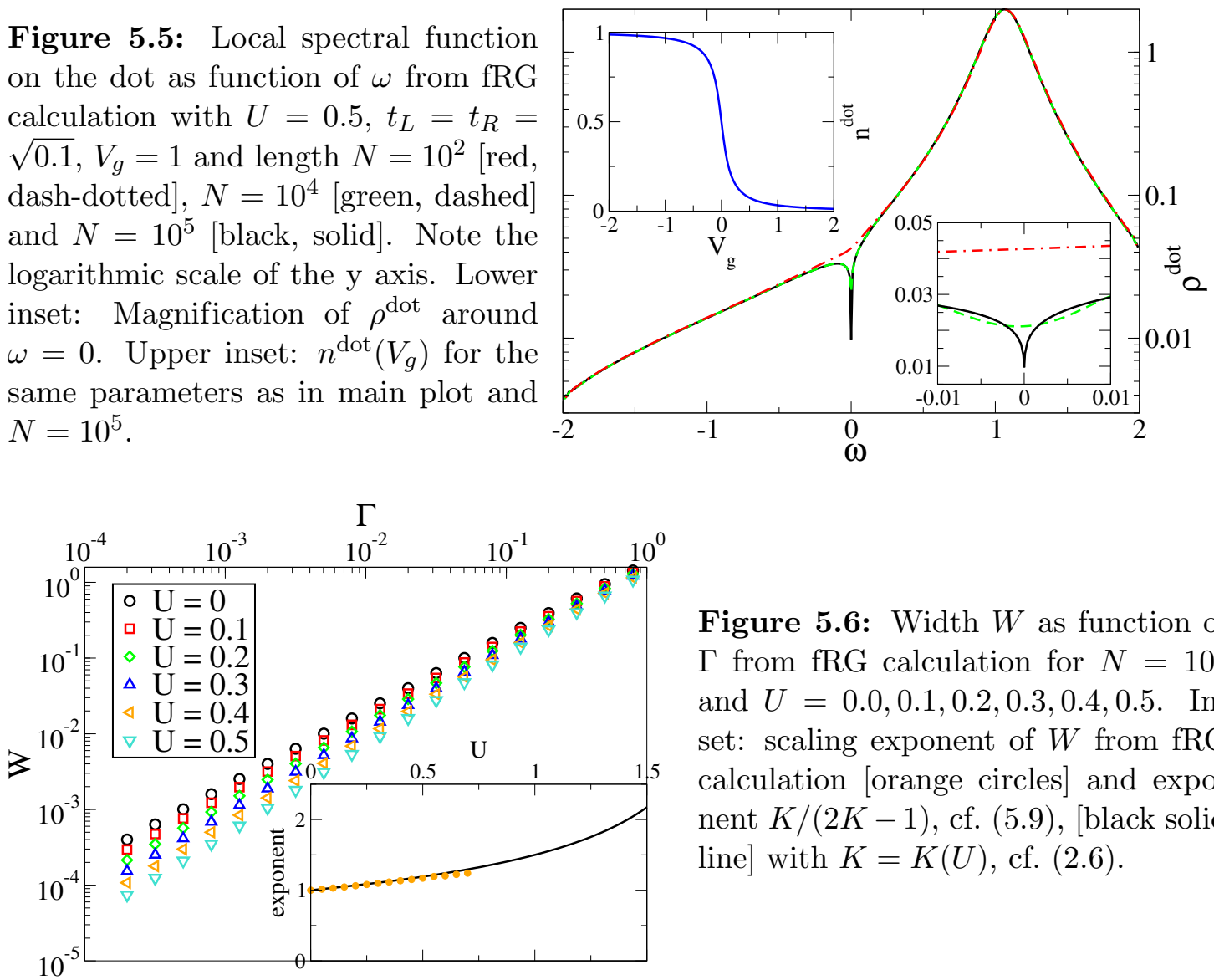

Figure 5.6: Width $W$ as function of $\Gamma$ from fRG calculation for $N=10^{5}$ and $U=0.0,0.1,0.2,0.3,0.4,0.5$. Inset: scaling exponent of $W$ from fRG calculation [orange circles] and exponent $K /(2 K-1)$, cf. $(5.9)$, [black solid line] with $K=K(U)$, cf. (2.6).

1 and $N=10^{2}, 10^{4}, 10^{5}$. The dominating feature is the Lorentz-like peak at $\omega \approx V_{g}$. Although the charge on the dot was assumed not to interact with the electrons in the leads, the coupling to the Luttinger-liquid leads induces the characteristic power-law suppression $\rho^{\operatorname{dot}}(\omega) \sim \omega^{1 / K-1}$, which develops with increasing $N$. The lower inset of fig. 5.5 shows a magnification of the dip region. This feature can also be found in the perturbative local spectral function (5.5). It is noteworthy, that the characteristic suppression is not observed for leads with $N \ll 10^{4}$ and is not developed properly even for $N \approx 10^{4}$, cf. the lower inset of fig. 5.5. This illustrates the general observation, that the energy scale at which the asymptotic low-energy behavior sets in is very low (cf. e. g. [MMSS02a, $\mathrm{AEM}^{+}$04] or even more severe in spinfull systems [AEM ${ }^{+}$06b]). The occasional attempt (e. g. [SWGB05, GWB09]) to numerically prove or disprove Luttinger-liquid behavior on significantly smaller systems has thus to be regarded with suspicion.

The suppression of $\rho^{\mathrm{dot}}$ also manifests in the charging $n^{\mathrm{dot}}\left(V_{g}\right)$ of the dot. To obtain $n^{\operatorname{dot}}\left(V_{g}\right)$ within the approximate fRG, two different schemes, viz. the integration of $\rho^{\operatorname{dot}}(\omega)$, eq. (3.22), and a flow equation, eq. (3.23), were introduced in section 3.3. In the description of the asymptotic behavior of $n^{\operatorname{dot}}\left(V_{g}\right)$, the latter scheme is expected to be superior [AEM ${ }^{+}$44, sec. III.B.2], however, it was checked that both approaches give identical asymptotic results. In the following the integration over $\rho^{\operatorname{dot}}(\omega)$ was used. The upper inset of fig. 5.5 exemplifies $n^{\operatorname{dot}}\left(V_{g}\right)$ on a system with 
parameters as in the main plot and $N=10^{5}\left(n^{\operatorname{dot}}\left(V_{g}\right)\right.$ does not change upon further increasing $N$ ).

Within the approximate fRG, the scaling formula $(5.9)$ for the width $W$ as function of $\Gamma$ can be confirmed by extracting $W$ from $n^{\operatorname{dot}}\left(V_{g}\right)$ for different $\Gamma$ and interactions $U$. For a system with $N=10^{5}$, the results are shown in the main plot of fig. 5.6 on a double-logarithmic scale ${ }^{5}$ At small $\Gamma$ power-law scaling can clearly be observed. The exponent (calculated by fitting the data exemplified in the main plot of fig. 5.6 is plotted in the inset of fig. 5.6 as function of $U$. For comparison, the function $K(U) /(2 K(U)-1)$ as obtained from the perturbative calculation, eq. (5.9), with $K(U)$ given by (2.6) is included. Good agreement is found for $0 \leq U \lesssim 1 / 2$. For larger $U$, higher order corrections from the interaction, neglected in the approximate fRG, cf. section 3.1 .2 become important.

\section{side note: the role of spin}

The choice of spinless fermions completely excludes any Kondo physics from the problem. Experimentally, this can be achieved by lifting the spin degeneracy of the dot level with a magnetic field or by going to temperatures greater than the Kondo temperature [COK98]. Given this absence of the Kondo effect, the inclusion of spin does not lead to new physics. The perturbative analysis in section 5.2 .2 can be adapted to the spinfull case by replacing the exponent $1 / K-1$ in the local spectral function (5.3) according to section 2.1. A study of the conductance in the presence of the Kondo effect can be found in [AEM06a].

\section{schematic proposal of experimental setup}

The power laws identified and explained in the two preceding subsections can be exploited to extract two different Luttinger-liquid exponents (say, $\alpha_{1}$ and $\alpha_{2}$ ) from two distinct observables using a single probe. Moreover, the two observables can be varied as functions of two different external parameters. In section 1.2 , this was identified as being of great use in the experimental observation of predicted Luttinger-liquid behavior. The appropriate experimental setup, already shown in fig. 1.2, is re-sketched in fig. 5.7; Electrodes define the gate voltage $V_{g}$ and the constrictions $\Gamma_{L}, \Gamma_{R}$, which control the coupling $\Gamma$. The charge on the dot is measured by a quantum point contact $\left[\mathrm{FSP}^{+} 93, \mathrm{SRI}^{+} 04\right]$ or a single-electron transistor electrometer [BZAS99, DLW ${ }^{+}$99].

Measuring the conductance as function of temperature at fixed gate voltage and constrictions gives a first exponent $\alpha_{1}=2 \alpha_{\text {end }}$ for $K<1$, cf. subsection 5.2.1.

A second exponent $\alpha_{2}$ can be extracted at fixed constrictions (thus fixed coupling) and fixed, sufficiently low temperature from the behavior of $n^{\text {dot }}$ upon vary-

\footnotetext{
${ }^{5}$ Note that for the fRG data, the width of $n^{\operatorname{dot}}\left(V_{g}\right)$ was defined as full width at half maximum of $\left|\frac{d n^{\mathrm{dot}}}{d V_{g}}\right|$. Concerning the scaling properties, this definition is equivalent to the one used in the derivation of eq. (5.9).
} 


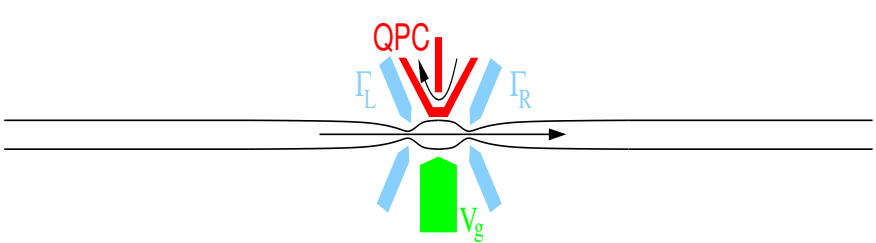

(a)

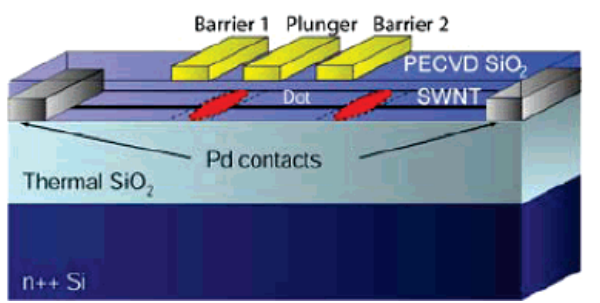

(b)

Figure 5.7: (a) Schematic of experimental setup to measure transport through the dot region as well as the charge on the dot. Electrodes define the gate voltage $V_{g}$ and the constrictions $\Gamma_{L}, \Gamma_{R}$. The charge on the dot is measured by a quantum point contact (QPC); (b) state-of-art experimental setup realizing SWNT based quantum dots controlled by electrostatic gates [adapted with permission from $\left[\mathrm{BGM}^{+} 05\right.$ ], copyright 2005 American Chemical Society].

ing $V_{g}$ close to resonance. Depending on the strength of the interaction of the electrons in the leads, either $\alpha_{2}=(2 K-1) /(1-K)=1 / \alpha_{\text {end }}-1$, cf. eq. (5.6), or $\alpha_{2}=1 / K-2=\alpha_{\text {end }}-1$, cf. eq. (5.8). Furthermore, an $\alpha_{2}$ can be obtained from measuring the width $W$ as function of the coupling $\Gamma$ at fixed gate voltage and fixed, sufficiently low temperature. This method has the advantage of being applicable over the whole range of not too strong interactions $(K>1 / 2)$, giving $\alpha_{2}=K /(2 K-1)=1 /\left(1-\alpha_{\text {end }}\right)$.

From the measured exponents, the Luttinger-liquid exponent $K$ can independently be calculated. A consistent result, $K\left(\alpha_{1}\right) \approx K\left(\alpha_{2}\right)$, would be a strong indication of Luttinger-liquid physics, cf. section 1.2 .

\section{side note: charging of a side-coupled dot (b) and end-coupled dot (c)}

In this side note, results on the charging of a dot from recent studies on the geometries shown in fig. 5.1 (b) and (c) are reviewed to provide some overview over the field. The geometries are either realized by coupling the level to a Luttinger liquid containing right-moving, as well as left-moving elementary excitations (i. e. the Luttinger liquid studied throughout this chapter, introduced in section 2.1) or to a Luttinger liquid merely containing elementary excitations propagating in a single direction. The former is termed non-chiral Luttinger liquid in this side note, the latter chiral Luttinger liquid. ${ }^{6}$

A single level, side-coupled to a non-chiral Luttinger liquid is studied in [LYY08] using a poor man's RG approach. No power-law behavior in the charging is found for weak interactions $(K \rightarrow 1)$, but only small corrections to the form for a noninteracting lead $(5.2)$. For very strong interactions $(K<2-\sqrt{3})$ the charging is conjectured to exhibit a jump of unit height at vanishing gate voltage (as in the case of a detached level). The point $V_{g}=0$ is approached as a power-law

\footnotetext{
${ }^{6}$ Compare with Cha03 for more details on chiral Luttinger liquids.
} 
$n\left(V_{g} \searrow 0\right) \sim V_{g}^{\alpha_{\text {bulk }}-1}$ and $n\left(V_{g} \nearrow 0\right) \sim 1-V_{g}^{\alpha_{\text {bulk }}-1}$ with $\alpha_{\text {bulk }}=K+1 / K-2$, cf. [LYY08, p. 4].

In [FM02], the charging of a single level, side-coupled to a chiral Luttinger liquid is studied by a perturbative expansion of the Green function of the dot to leading order in the coupling. This approach is valid for $0<K<1 / 2$ and provides in this regime the same results as obtained in section 5.2 .2 for the single level with two non-chiral Luttinger-liquid leads, cf. eq. (5.7). By mapping the system onto the anisotropic Kondo model, it is argued from the scaling behavior of the model [TW83, p. 593 et seq.], that for any $\Gamma$ a $K^{*} \leq 1 / 2$ exists above which the charging becomes continuous, cf. [FM02, p. 3]. In particular, if $\Gamma \ll 1, K^{*} \approx 1 / 2$. Furthermore, the single level, side-coupled to the chiral Luttinger liquid is identified as being equivalent to a single level, end-coupled to a non-chiral Luttinger liquid, if the levelLuttinger-liquid coupling is weak.

In GWB09 such a single level, end-coupled to a non-chiral Luttinger liquid (cf. fig. 5.1 (c)) is studied by expanding the partition function and casting it into the form of the Coulomb gas. Using the known connection to the Kondo model (cf. [AG69] and [TW83, p. 495 et seq.]), and the scaling results for the anisotropic Kondo model (cf. [TW83, p. 593 et seq.], similar to [FM02]), it was argued, that for $\Gamma \ll 1$ and $K<1 / 2$, the results of FM02 hold, viz. the charging function is discontinuous with power-law behavior given by (5.8) for $K<1 / 2$. The results from [TW83, p. 593 et seq.] furthermore imply, that in the regime $K>1 / 2$ the charging is linear in leading order in $V_{g}$ and that the characteristic width $W$ around $V_{g}=0$ over which the charge on the dot changes from unity to zero exhibits power-law behavior in the coupling, similar to eq. (5.9).7

Overall, in each model, cf. fig. 5.1 (a)-(c), with chiral or non-chiral Luttinger liquids, (at least) one regime in the interaction was identified for which $n\left(V_{g} \rightarrow 0\right)$ exhibits power-law scaling with an exponent depending only on the Luttinger liquid parameter $K{ }^{8}$

\footnotetext{
${ }^{7}$ This obviously contradicts the results of [SWGB05]. Most likely, the contradiction originates in the length of the system used in the DMRG calculations, which is insufficient to reach any Luttinger-liquid behavior, cf. the discussion of the developing Luttinger-liquid suppression in the local spectral function on page 58 and the discussions in $\mathrm{AEM}^{+} 04$, MMSS02a.

${ }^{8}$ In this respect, the statement «...that LL [Luttinger liquid] physics ... cannot be manifested through the behavior of any of the thermodynamic properties...» GWB09, p. 5] is rather surprising.
} 


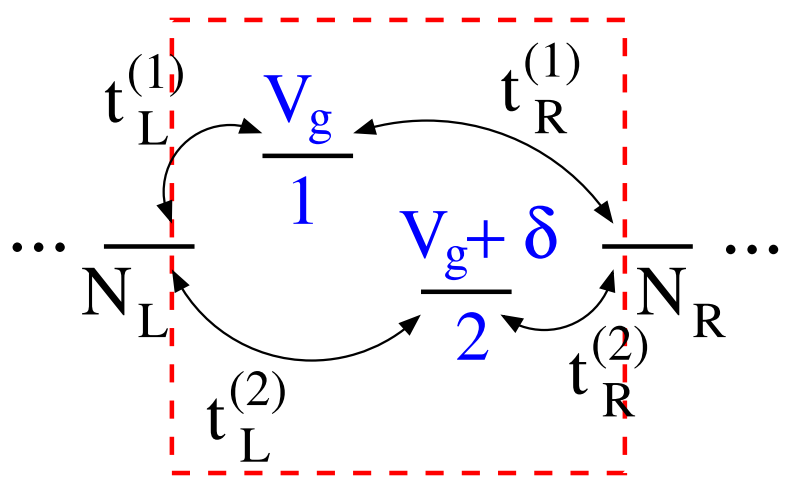

Figure 5.8: Parallel double quantum dot: Two energy levels (dot 1 and dot 2) coupled in parallel to the two leads with nearest-neighbour hopping terms $t_{L}^{(1)}, t_{R}^{(2)}$ and next-nearestneighbour hopping terms $t_{L}^{(2)}, t_{R}^{(1)}$. The charge on the dots is chosen not to interact with the electrons in the leads. Red, dashed box as in fig. 5.2 .

\section{parallel double dot with two Luttinger-liquid leads (d)}

A parallel double quantum dot is implemented in the model introduced in section 5.1. eq. (5.1). The explicit choice of the Hamiltonian $h^{\operatorname{dot}}\left(\left\{d^{\dagger}, d\right\}\right)$ reads

$$
h^{\mathrm{dot}}=V_{g} d_{1}^{\dagger} d_{1}+\left(V_{g}+\delta\right) d_{2}^{\dagger} d_{2}-\sum_{\substack{j=1,2 \\ a=L, R}} t_{a}^{(j)} d_{j}^{\dagger} c_{N_{a}}+\text { H.c. } .
$$

Fig. 5.8 illustrates the implementation in the general fRG scheme of section 5.1 The last site $N_{L}$ of the left lead (cf. also fig. 5.2) is connected to dot 1 via a nearest-neighbour hopping $t_{L}^{(1)}$ and to dot 2 via a next-nearest-neighbour hopping $t_{L}^{(2)}$. Similarly, the last site $N_{R}$ of the right lead is connected to the dots by the hoppings $t_{R}^{(1)}$ (next-nearest neighbour) and $t_{R}^{(2)}$ (nearest neighbour). As the nearestneighbour hoppings introduce elements on the first off-diagonal in the Hamiltonian matrix, the next-nearest-neighbour hoppings introduce elements on the second offdiagonal. Therefor, the full matrix embedded between the two long tridiagonal parts in the inverse Green function is of size $(4 \times 4)$, cf. also fig. 5.2. There is no interaction between the charge on the dots and the electrons in the leads. For simplicity, $N_{L}=N_{R}=N$ is chosen and the leads are half-filled.

Figs. 5.9 to 5.13 illustrate the influence of the interaction $U$ in the leads on the conductance $G\left(V_{g}\right)$ through the wire-dot system. The case of non-interacting leads is reviewed in appendix $\mathrm{G}$.

The three basic forms (cf. appendix $\mathrm{G}$ ) of $G\left(V_{g}\right)$ for tuned (i. e. $\delta=0$ ) dots, viz. resonance at $V_{g}=0$ [blue curves] $\left(t_{L}^{(1)}=t_{R}^{(1)}\right.$ and $\left.t_{L}^{(2)}=t_{R}^{(2)}\right)$, anti-resonance at $V_{g}=0$ [red curves] $\left(t_{L}^{(1)} t_{R}^{(2)}-t_{L}^{(2)} t_{R}^{(1)} \neq 0\right)$ and non-vanishing $G / G_{0}<1$ at $V_{g}=0$ [black curves] $\left(t_{L}^{(1)} t_{R}^{(2)}-t_{L}^{(2)} t_{R}^{(1)}=0\right.$ ) are shown in fig. 5.9 for different interactions $U$. Each lead is of length $N=1111$. For any set of parameters, except on resonance, the conductance gets suppressed for all $V_{g}$ with increasing interaction. On resonance the conductance stays pinned to $G_{0}$ and the width of the resonance peak decreases as $G$ is suppressed for any $V_{g} \neq 0$. Fig. 5.10 shows the dependence of $G$ on the length of the leads $N$ (i. e. the finite-size scaling of the conductance) at fixed interaction $U=0.5$ and gate voltage $V_{g}$ with couplings 
Figure 5.9: Conductance $G$ as function of gate voltage $V_{g}$ through tuned $(\delta=0)$ parallel double quantum dots coupled to the leads $(N=1111)$ with $t_{L}^{(1)}=t_{R}^{(1)}=t_{L}^{(2)}=t_{R}^{(2)}=0.3$ [blue lines], $t_{L}^{(1)}=0.4, t_{R}^{(1)}=0.3, t_{L}^{(2)}=$ $0.2, t_{R}^{(2)}=0.15$ (i. e. $t_{L}^{(1)} t_{R}^{(2)}-t_{L}^{(2)} t_{R}^{(1)}=$ $0)$ [black lines] and $t_{L}^{(1)}=0.4, t_{R}^{(1)}=$ $0.3, t_{L}^{(2)}=0.2, t_{R}^{(2)}=0.1$ (i. e. $t_{L}^{(1)} t_{R}^{(2)}-$ $\left.t_{L}^{(2)} t_{R}^{(1)} \neq 0\right)$ [red lines]. From top to bottom the interaction $U$ in the leads is raised as indicated in the plot.
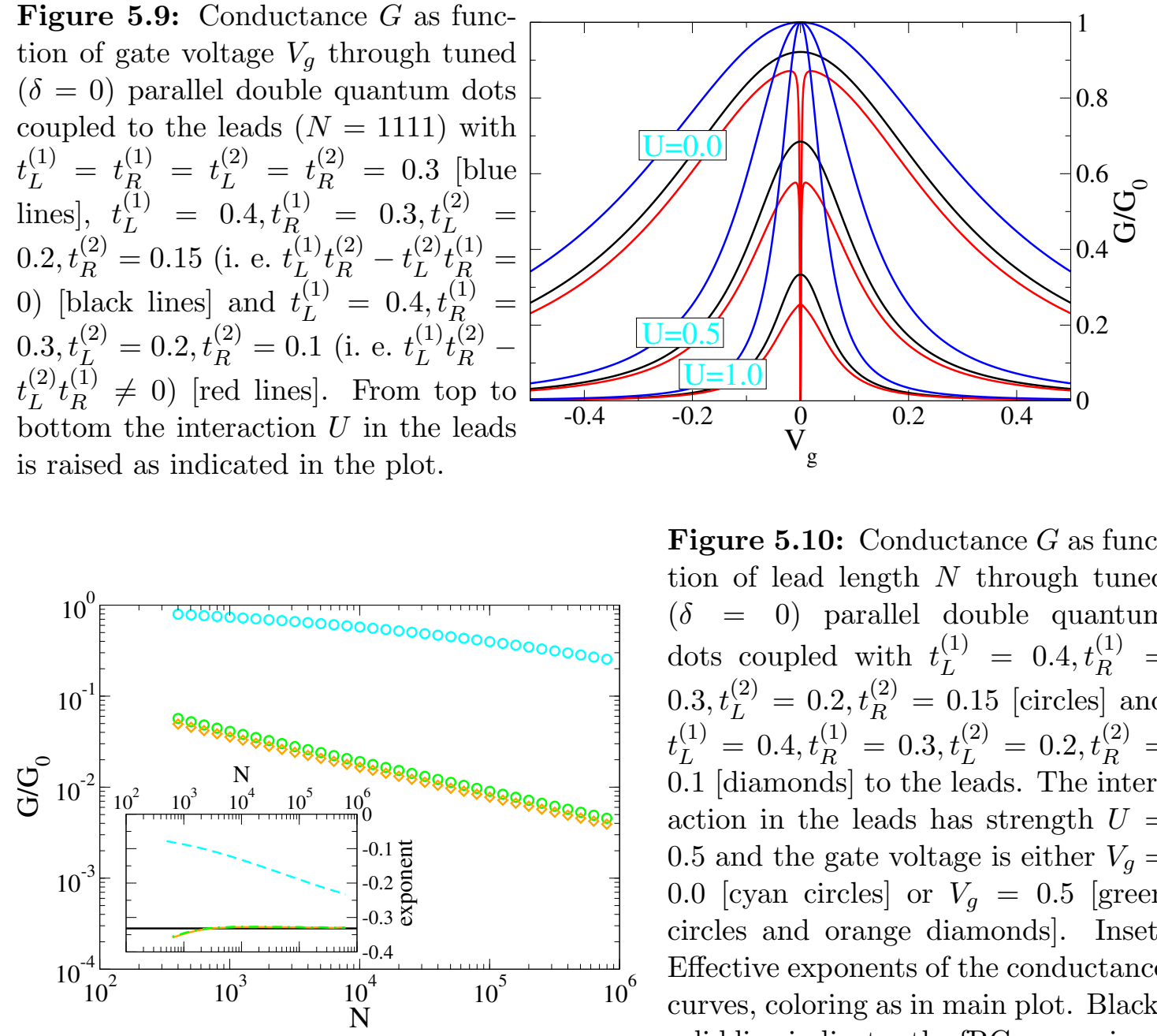

Figure 5.10: Conductance $G$ as function of lead length $N$ through tuned $(\delta=0)$ parallel double quantum dots coupled with $t_{L}^{(1)}=0.4, t_{R}^{(1)}=$ $0.3, t_{L}^{(2)}=0.2, t_{R}^{(2)}=0.15$ [circles] and $t_{L}^{(1)}=0.4, t_{R}^{(1)}=0.3, t_{L}^{(2)}=0.2, t_{R}^{(2)}=$ 0.1 [diamonds] to the leads. The interaction in the leads has strength $U=$ 0.5 and the gate voltage is either $V_{g}=$ 0.0 [cyan circles] or $V_{g}=0.5$ [green circles and orange diamonds]. Inset: Effective exponents of the conductance curves, coloring as in main plot. Black, solid line indicates the fRG approximation for $-2 \alpha_{\text {end }}$, cf. $\left[\mathrm{EMA}^{+} 05\right.$.

$t_{L}^{(1)}=0.4, t_{R}^{(1)}=0.3, t_{L}^{(2)}=0.2, t_{R}^{(2)}=0.15$ (the basic form shown by the black lines in fig. 5.9) and $t_{L}^{(1)}=0.4, t_{R}^{(1)}=0.3, t_{L}^{(2)}=0.2, t_{R}^{(2)}=0.1$ (the basic form shown by the red lines in fig. 5.9p. For high $\left|V_{g}\right|$, the conductance scales with a power law for $N \gtrsim 10^{3}$ [green squares and orange diamonds; $V_{g}=0.5$ ]. The green and orange curves in the inset show, that the exponents of the power laws reach an asymptotic value of $-2 \alpha_{\text {end }}=-2(1 / K-1)$, determined by the Luttinger-liquid parameter $K(U)$ of the leads, cf. (2.6). For low $\left|V_{g}\right|$, the conductance curve trends towards an asymptotic power law [cyan circles; $V_{g}=0.0$ ], as is clearly indicated by the trend in the exponent [cyan curve in the inset]. The asymptotic low-energy behavior is reached for lead lengths beyond $10^{8}$. Thus, for any set of parameters and gate voltage, except on resonance (to be discussed below), the conductance $G$ vanishes asymptotically in the low-energy limit, scaling $\sim N^{-2 \alpha_{\text {end }}}$.

To complement this result, the local spectral function $\rho(\omega)$ on site $N_{L}$, i. e. to the left of the double-dot structure, is presented in fig. 5.11 . For both cases, viz. $t_{L}^{(1)}=$ $0.4, t_{R}^{(1)}=0.3, t_{L}^{(2)}=0.2, t_{R}^{(2)}=0.1$ [solid lines] and $t_{L}^{(1)}=0.4, t_{R}^{(1)}=0.3, t_{L}^{(2)}=$ 


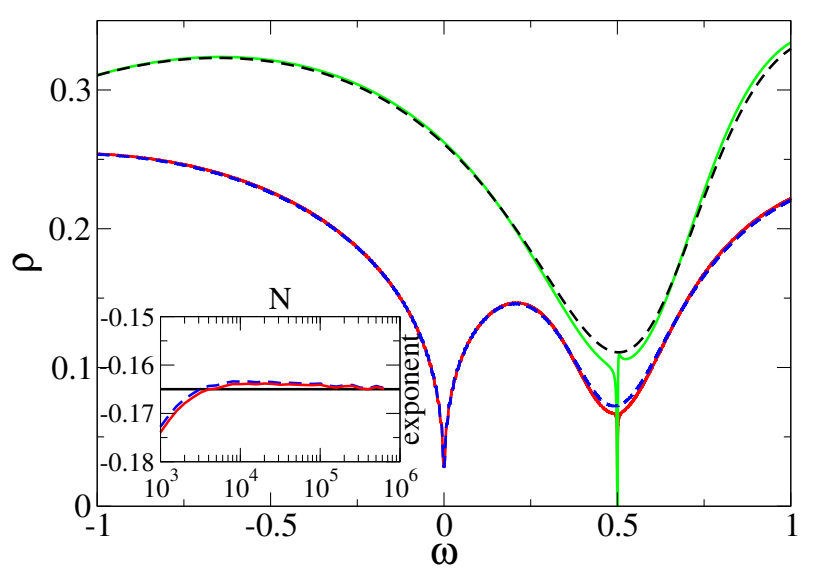

Figure 5.11: Local spectral function $\rho(\omega)$ on the site $N_{L}$ for tuned $(\delta=$ $0)$ parallel double quantum dots coupled with $t_{L}^{(1)}=0.4, t_{R}^{(1)}=0.3, t_{L}^{(2)}=$ $0.2, t_{R}^{(2)}=0.1\left[\right.$ solid lines] and $t_{L}^{(1)}=$ $0.4, t_{R}^{(1)}=0.3, t_{L}^{(2)}=0.2, t_{R}^{(2)}=0.15$ [dashed lines] to the leads $(N=1111)$. The gate voltage is $V_{g}=0.5$, interaction in the leads has strength $U=$ 0.0 [green, black] and $U=0.5$ [red, blue]. The $(U=0)$-curve was calculated with G.1), the $(U=0.5)$-curve with the approximate fRG. Only part of the band is shown to better resolve the low-energy features. Inset: exponent of suppression at $\omega=0$ as function of length $N$, coloring as in main plot.

$0.2, t_{R}^{(2)}=0.15$ [dashed lines] at $V_{g}=0.5$, a suppression at $\omega=0$ develops for nonvanishing interaction $U>0$ in the leads. The finite size scaling of $\rho(\omega=0)$ shows, that the suppression asymptotically develops into a power law with exponent $-\alpha_{\text {end }}$ (cf. inset). Thus, the form of the local spectral function around $\omega=0$ is similar to that of the local spectral function close to an open boundary in a Luttinger liquid [FG95, $\mathrm{MMS}^{+}$00] or equivalently next to an isolated impurity [KF92b, MMSS02a, $\mathrm{AEM}^{+}$04]. Similar results hold for the local spectral function to the right, i. e. on site $N_{R}$. The scaling behavior $\sim N^{-2 \alpha_{\text {end }}}$ of the conductance can then be understood by Fermi's Golden Rule, cf. [BF04, p. 86] and chapter 4, which states, that the tunneling rate (and thus the conductance, cf. eq. (3.19) ) for an electron to tunnel from the left-hand side of the dot structure to the right-hand side is proportional to the product of the density of states on the left hand and right-hand side. Each of these scales $\sim N^{-\alpha_{\text {end }}}$ as seen above. The features of the local spectral functions around $\omega=V_{g}$ are unrelated to the interaction of the electrons in the leads and explained in appendix $\mathrm{G}$. Only part of the band is shown in the figure to better resolve the low-energy features.

For tuned dots, individually coupled symmetrically (i e. $t_{L}^{(1)}=t_{R}^{(1)}$ and $t_{L}^{(2)}=t_{R}^{(2)}$ ) to the leads, the conductance was found to exhibit a resonance peak around $V_{g}=0$, where $G / G_{0}=1$ (cf. blue curves in fig. 5.9). In fig. 5.12, the finite-size scaling behavior of the conductance for couplings $t_{L}^{(1)}=t_{R}^{(1)}=t_{L}^{(2)}=t_{R}^{(2)}=0.1$ is shown. On resonance, i. e. at $V_{g}=0$, the conductance stays pinned to $G_{0}$ for any $N$ and interaction strength $U \leq 2$ (exemplified at $U=0.5$ [red crosses] and $U=1.99$ [blue circles]). The exponent in the inset also implies, that $G\left(V_{g}=0\right)$ does not depend on $N$. However, for any $V_{g} \neq 0$, i. e. off resonance, the conductance is suppressed $\sim N^{-2 \alpha_{\text {end }}}$ similarly to the case of asymmetrically coupled dots discussed above 
Figure 5.12: Conductance $G$ as function of lead length $N$ through tuned $(\delta=0)$ parallel double quantum dots coupled with $t_{L}^{(1)}=t_{R}^{(1)}=t_{L}^{(2)}=t_{R}^{(2)}=$ 0.1 to the leads. On resonance $\left(V_{g}=0\right)$ with $U=0.5$ [red crosses] and $U=$ 1.99 [blue circles], off resonance $\left(V_{g}=\right.$ $0.5)$ with $U=0.5$ [green triangles]. Inset: Effective exponents of the conductance curves, coloring as in main plot. Black, solid line indicates the fRG ap- $10^{2}$ proximation for $-2 \alpha_{\text {end }}$, cf. [ $\left.\mathrm{EMA}^{+} 05\right]$.

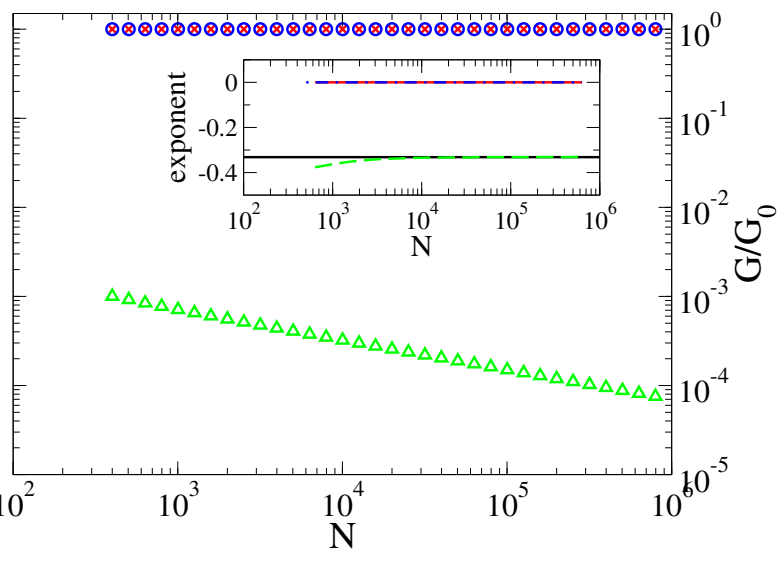

(exemplified at $U=0.5$ and $V_{g}=0.5$ [green triangles]). Therefor, the width of the resonance peaks vanishes.

Summarizing the results on the conductance and local spectral function for tuned dots, the asymptotic low-energy behavior is found to be governed by the same fixed points as the double-barrier problem, whose conductance was already discussed in the context of a single dot with two Luttinger-liquid leads, cf. section 5.2.1 ${ }^{9}$ To be specific, no traces of any fixed point, apart from the "perfect-chain" fixed point, asymptotically reached on resonance, and the "open-chain" fixed point, reached off resonance, could be observed in the data. On first glance, this seems to contradict the work of [RS04], who identified an intermediate fixed point, realized for $t_{L}^{(1)}=$ $t_{R}^{(1)}=t_{L}^{(2)}=t_{R}^{(2)}, K \searrow 1 / 2$ (i. e. $U \rightarrow 2$, cf. (2.6)) and $V_{g}=0$. The fixed point is characterized by a non-vanishing and non-perfect conductance $G / G_{0} \sim 1-1 /(2 K)$ [RS04, eq. (25)]. However, the apparent contradiction can be resolved by taking a closer look at the model studied. As done in [NFLL04] in modelling a single dot, the two dot levels ( 1 and 2) were represented by spin-1/2 raising and lowering operators $S_{j=1,2}^{ \pm}$. These operators anti-commute on the same site $\left(j_{1}=j_{2}\right)$, but commute on differing sites $\left(j_{1} \neq j_{2}\right)$. This model, thus, does not describe two fermionic levels, in contrast to the Hamiltonian $h^{\text {dot }}$, cf. eq. (5.10) ${ }^{10}$

${ }^{9}$ The results presented there for $T \gg \pi v_{F} / N$ have to be adapted by replacing $T$ with $N^{-1}$, as for $T=0$ and finite $N$, as used here, $N^{-1}$ constitutes the generic low-energy cutoff, cf. also $\mathrm{EMA}^{+} 05$ and section 2.1 .

${ }^{10}$ Correct fermionic anti-commutation relations can be obtained for the spin operators, replacing $S_{2}^{+}$by $\tilde{S}_{2}^{+} \rightarrow e^{-\imath \pi S_{1}^{+} S_{1}^{-}} S_{2}^{+}$and $S_{2}^{-}$accordingly (Jordan-Wigner transformation, e. g. [NO98, p. 434]). In [RS04, the fixed point structure of the model with the pure spin operatots $S_{1 / 2}^{ \pm}$ is studied by mapping the partition function onto a generalized Coulomb gas as done for the double-barrier problem in KF92a, KF92b. The differences arise from relative phases entering the partition function due to the presence of two levels (instead of one level as in KF92a, KF92b]), which introduce an additional non-vanishing "magnetic field" $\beta$ in the Coulomb gas (leading to the dissipative Hofstadter model [COA03, CJF92, CJKMY95]). Sample calculations along this reasoning of RS04 but with the operators $\tilde{S}_{2}^{ \pm}$show, that the additional relative phases from the phase operators in $\tilde{S}_{2}^{ \pm}$exactly cancel the additional relative phases due to the presence of the second level, leading back to a vanishing $\beta$. Thus, one may tentatively conjecture, that the introduction of fermionic operators $\tilde{S}_{2}^{ \pm}$leads to a reproduction of the results from the double-barrier problem. 
Figure 5.13: Conductance $G$ as function of gate voltage $V_{g}$ through detuned $(\delta=0.5)$ parallel double quantum dots coupled to the leads $(N=$ 1111) with $t_{L}^{(1)}=t_{R}^{(1)}=0.1, t_{L}^{(2)}=$ $t_{R}^{(2)}=0.2[$ solid lines] and interaction $U=0$ [green] and $U=0.5$ [cyan]. Alike but with $t_{L}^{(1)}=0.4, t_{R}^{(1)}=$ $0.3, t_{L}^{(2)}=0.2, t_{R}^{(2)}=0.1 \quad[$ dashed lines] and interaction $U=0$ [red] and $U=0.5$ [orange]. Inset: exponent extracted from a finite size scaling (cf. e. g. fig. 5.10) of $G$ at $V_{g}=0.5$,

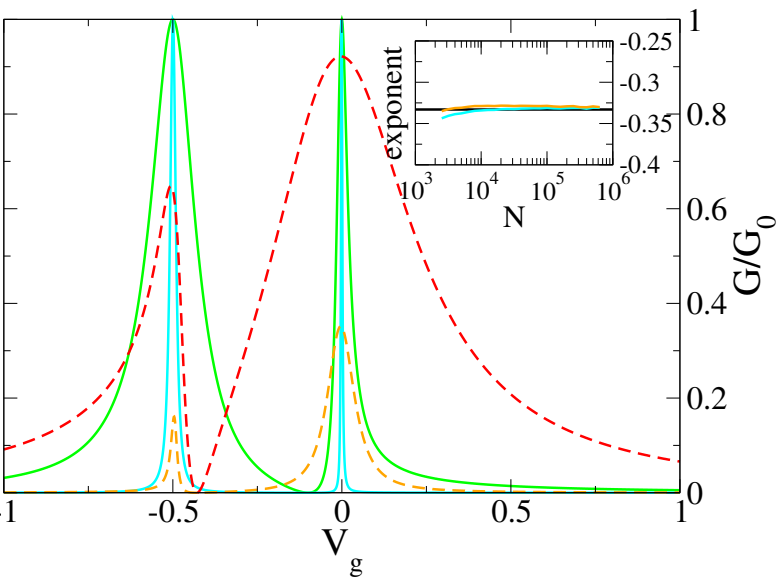
coloring as in main plot.

The case of detuned $(\delta \neq 0)$ dots is exemplified in figs. 5.13 and 5.14 . Fig. 5.13 compares the conductance $G\left(V_{g}\right)$ with detuning $\delta=0.5$ for non-interacting and interacting leads. It generically shows two pronounced peaks around gate voltages $V_{g}=0$ and $V_{g}=-\delta$. For dots, which are individually coupled symmetrically to the leads, the maximum of the peaks is $G / G_{0}=1$, irrespective of the interaction in the leads [solid lines with couplings $t_{L}^{(1)}=t_{R}^{(1)}=0.1$ and $t_{L}^{(2)}=t_{R}^{(2)}=0.2$ ]. For gate voltages away from resonance, i. e. any $V_{g}$ with $G\left(V_{g}\right) / G_{0}<1$ for noninteracting leads, the conductance is suppressed in the case of interacting leads, scaling $\sim N^{-2 \alpha_{\text {end }}}$ [cf. inset]. This leads to infinitely sharp resonance peaks in the low-energy limit.

Any asymmetry in the couplings leads to conductance maxima $G / G_{0}<1$, even in the absence of interactions in the leads, cf. eq. G.2a), and to the asymptotic suppression $G \sim N^{-2 \alpha_{\text {end }}}$ for any $V_{g}$. This is exemplified in fig. 5.13 for couplings $t_{L}^{(1)}=0.4, t_{R}^{(1)}=0.3, t_{L}^{(2)}=0.2, t_{R}^{(2)}=0.1$ by the dashed lines [red: $U=0$; orange: $U=0.5]$. The exponent of the suppression as function of $N$ is shown in the inset at $V_{g}=0.5$. For $N \gtrsim 10^{4}$, the asymptotic value of $-2 \alpha_{\text {end }}$ is reached.

The local spectral function on the dots (1 [red line] and 2 [blue line]) and on the site $N_{L}$ [green line] to the left of the dot structure is shown in fig. 5.14 for detuning $\delta=0.5$, asymmetric couplings $t_{L}^{(1)}=0.1, t_{R}^{(1)}=0.1, t_{L}^{(2)}=0.1, t_{R}^{(2)}=0.15$ and $V_{g}=-0.25$. The interaction in the leads $(N=1111)$ has strength $U=0.5$. On each dot, the local spectral function shows a pronounced peak around the eigenenergy of the isolated level (i. e. at $\omega=V_{g}=-0.25$ for $\operatorname{dot} 1$ and $\omega=V_{g}+\delta=0.25$ for dot 2) [cf. right inset]. At the eigenenergy of the other dot, each local spectral function shows a dip, which is not related to the correlations in the leads, cf. appendix $\mathrm{G}$. At $\omega=0$, the local spectral function is suppressed for interacting leads $(U>0)$ and shows finite size scaling $\sim N^{-\alpha_{\text {end }}}$ [cf. left inset] similar to the case of a single dot, cf. fig. 5.5. The local spectral function to the left (and equally to the right) of the dot, exhibits a dip at each eigenenergy of the isolated dots, and the correlations in 


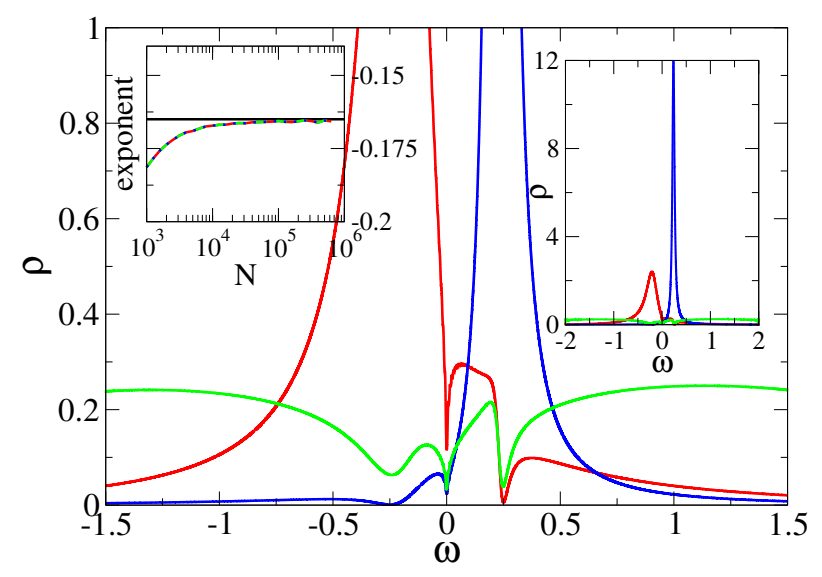

Figure 5.14: Local spectral function $\rho(\omega)$ of $\operatorname{dot} 1$ [red], dot 2 [blue] and site $N_{L}$ [green] for detuned $(\delta=$ $0.5)$ parallel double quantum dots coupled with $t_{L}^{(1)}=0.1, t_{R}^{(1)}=0.1, t_{L}^{(2)}=$ $0.1, t_{R}^{(2)}=0.15$ to the leads $(N=$ 1111). Gate voltage is $V_{g}=-0.25$, interaction in the leads has strength $U=0.5$. Only part of the band is shown in the figure to better resolve the low-energy features. Left inset: exponent extracted from a finite size scaling (cf. e. g. fig. 5.10) of the suppression at $\omega=0$, coloring as in main plot. Right inset: overview over entire local spectral functions, coloring as in main plot

the leads induce the asymptotic suppression $\rho(\omega=0) \sim N^{-\alpha_{\text {end }}}$. Thus, the behavior of the conductance can be explained similarly as in the case of tuned dots.

These examples show, that detuning does not change the physical picture explained above for tuned dots. The low-energy transport properties of the parallel double dot coupled to Luttinger-liquid leads are similar to the asymptotic behavior found in the problem of a double barrier embedded in a Luttinger liquid, cf. the references given in sec. 5.2 .1 .

\section{side note: dot-lead interaction within the approximate fRG}

Throughout the chapter, the charge on the $\operatorname{dot}(\mathrm{s})$ was assumed not to interact with the electrons in the leads. Physically, this seems legitimate as long as the dot-lead couplings are kept small. The technical reason, however, is that the implementation of dot-Luttinger-liquid-lead geometries within the fRG scheme presented in section 5.1 usually involves more-than-next-nearest-neighbour hoppings, for which no flow equations exist within the parametrization of the two-particle vertex, eq. (3.12). An exception are dots, exclusively coupled in series [EMA $\left.{ }^{+} 05\right]$.

In this side note, a single level, side-coupled to the bulk of a Luttinger-liquid lead is presented as special case, in which a dot-lead interaction can be treated within the fRG scheme of section 5.1 without adapting (3.12). The influence of this interaction on the conductance is briefly studied.

The Hamiltonian of the system explicitly reads

$$
\begin{aligned}
H^{\text {side }}= & H^{\text {wire }}+h_{L}^{\text {coupl }}+h_{R}^{\text {coupl }}+h_{L}^{1 \mathrm{~d} \text {-lead }}+h_{R}^{1 \mathrm{~d} \text {-lead }} \\
& +V_{g} d^{\dagger} d-t_{d} d^{\dagger} c_{N_{K}}+\text { H.c. }+U_{\mathrm{d}-1} d^{\dagger} d c_{N_{K}}^{\dagger} c_{N_{K}}
\end{aligned}
$$

The length $N$ is chosen to be odd and $N_{K}=(N+1) / 2$. The coupling to the non-interacting leads is perfect. Thus, the system is symmetric around $N_{K}$. 


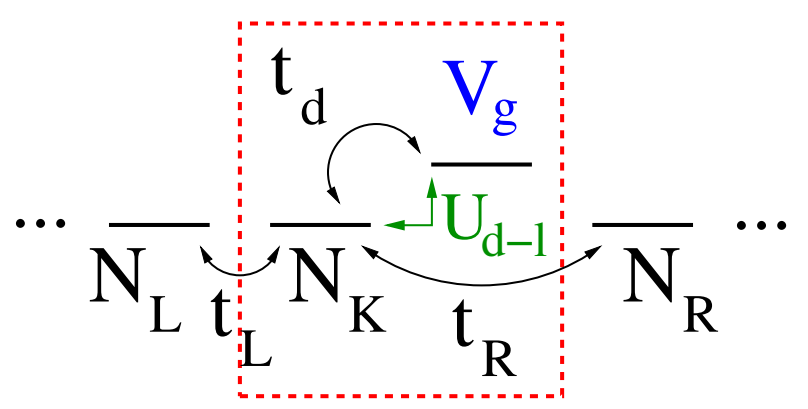

Figure 5.15: Side-coupled quantum dot: energy-level coupled to wire at site $N_{K}$ with hopping strength $t_{d}$. The charge on the dot is chosen to interact with the charge on $N_{K}$ with strength $U_{\mathrm{d}-1}$. Red, dashed box as in fig. 5.2 .

Fig. 5.15 illustrates the implementation in the scheme of section 5.1. The single level is coupled via a nearest-neighbour hopping term of strength $t_{d}$ to the site $N_{K}$, which is coupled by $t_{L / R}$ to $N_{L}$ and $N_{R}$ respectively. The Hamiltonian (5.11) implies $N_{L}=N_{R}=(N-1) / 2, t_{L}=t_{R}=t$. The coupling $t_{L}$ is a nearest-neighbour hopping, while $t_{R}$ is a next-nearest-neighbour hopping in the implementation and is not renormalized during the fRG flow. Therefor it would act as a hopping impurity, which breaks the symmetry. This problem can be circumvented by setting $\Sigma^{\Lambda}\left(N_{K}, N_{R}\right)=\Sigma^{\Lambda}\left(N_{L}, N_{K}\right)$ and thus $t_{R}^{\Lambda}=t+\Sigma^{\Lambda}\left(N_{K}, N_{R}\right)=t_{L}^{\Lambda}$ at each stage of the fRG flow, thereby mimicking the nearest-neighbour hopping $t_{R}$ and the nearestneighbour interaction $U$ between $N_{K}$ and $N_{R}$ by use of the symmetry properties.

For $U_{\mathrm{d}-1}=0$ and $t_{d}=0$, the dot is detached. For $U_{\mathrm{d}-1} \neq 0$, eqs. (3.14a) and $(3.14 \mathrm{~b})$ can locally be adapted to include the renormalization of the (nearestneighbour) hopping $t_{d}$, the gate voltage $V_{g}$ and the on-site energy of $N_{K}$ due to $U_{\mathrm{d}-1}$, giving $\left(j_{d}\right.$ labels the site of the dot)

$$
\begin{aligned}
\partial_{\Lambda} \Sigma^{\Lambda}\left(j_{d}, j_{d}\right)= & -\frac{U_{\mathrm{d}-1}}{\pi} \mathfrak{R e} \tilde{\mathcal{G}}^{\Lambda}\left(j_{d}, j_{d}, \imath \Lambda\right) \\
\partial_{\Lambda} \Sigma^{\Lambda}\left(N_{K}, N_{K}\right)= & -\frac{1}{\pi} \mathfrak{R e}\left[U^{\Lambda}\left(\tilde{\mathcal{G}}^{\Lambda}\left(N_{L}, N_{L}, \imath \Lambda\right)+\tilde{\mathcal{G}}^{\Lambda}\left(N_{R}, N_{R}, \imath \Lambda\right)\right)\right. \\
& \left.\quad+U_{\mathrm{d}-1} \tilde{\mathcal{G}}^{\Lambda}\left(j_{d}, j_{d}, \imath \Lambda\right)\right] \\
\partial_{\Lambda} \Sigma^{\Lambda}\left(j_{d}, N_{K}\right)= & \frac{U_{\mathrm{d}-1}}{\pi} \mathfrak{R e} \tilde{\mathcal{G}}^{\Lambda}\left(j_{d}, N_{K}, \imath \Lambda\right) .
\end{aligned}
$$

Note that $U_{\mathrm{d}-1}$ itself is not renormalized, since 3.13 only applies to the interaction $U$ in the wire.

The main plot of fig. 5.16 shows the fRG flow of the on-site energy $\varepsilon_{N_{K}}$ of the coupling site $N_{K}$ in the wire [open symbols], the hopping $t_{d}$ [lines] and the on-site energy of the $\operatorname{dot}\left(V_{g}\right)$ [filled symbols] for a system with $U=0.5, V_{g}=-1.0$, length $N=7959$, dot-lead interaction $U_{\mathrm{d}-1}=3.0$ and $t_{d}=0.1$ [green symbols and lines], $t_{d}=0$ [black symbols and lines] respectively. The dot-lead hopping is only slightly renormalized if initially not zero and stays zero otherwise. However, the renormalization of $V_{g}$ and $\varepsilon_{N_{K}}$ does not depend on the initial $t_{d}$. In particular, $\varepsilon_{N_{K}}$ flows from 0 to a value $\approx 1.77$ even if $t_{d}=0$ initially. The impact on the self-energy of the wire is shown in the inset of fig. 5.16. For comparison, the case $U_{\mathrm{d}-1}=0$ (homogeneous wire) is included [black lines]: the diagonal $\Sigma_{j, j}$ stays zero (cf. section 4.1: interacting electrons) and the off-diagonal elements $\Sigma_{j, j+1}$ are constant. For 
Figure 5.16: Flow of on-site energy $\varepsilon_{N_{K}}$ of site $N_{K}$ [open symbols], on-site energy of $\operatorname{dot}\left(V_{g}\right)$ [filled symbols] and hopping $t_{d}$ [lines] for flow parameter $\Lambda: 10^{8} \rightarrow 10^{-4}$. Black: $t_{d}=0, U_{\mathrm{d}-1}=$ $3.0, U=0.5$ and $V_{g}=-1.0$; green: $t_{d}=0.1, U_{\mathrm{d}-1}=3, U=0.5$ and $V_{g}=$ -1 . Inset: Self-energy $\Sigma_{j, j}$ and $\Sigma_{j, j+1}$ at the end of the fRG flow $(\Lambda=0)$ of a wire with $U=0.5, t_{d}=0, V_{g}=-1$, $N=7959, N_{K}=3980$ and $U_{\mathrm{d}-1}=0$ [black], $U_{\mathrm{d}-1}=3.0$ [blue]
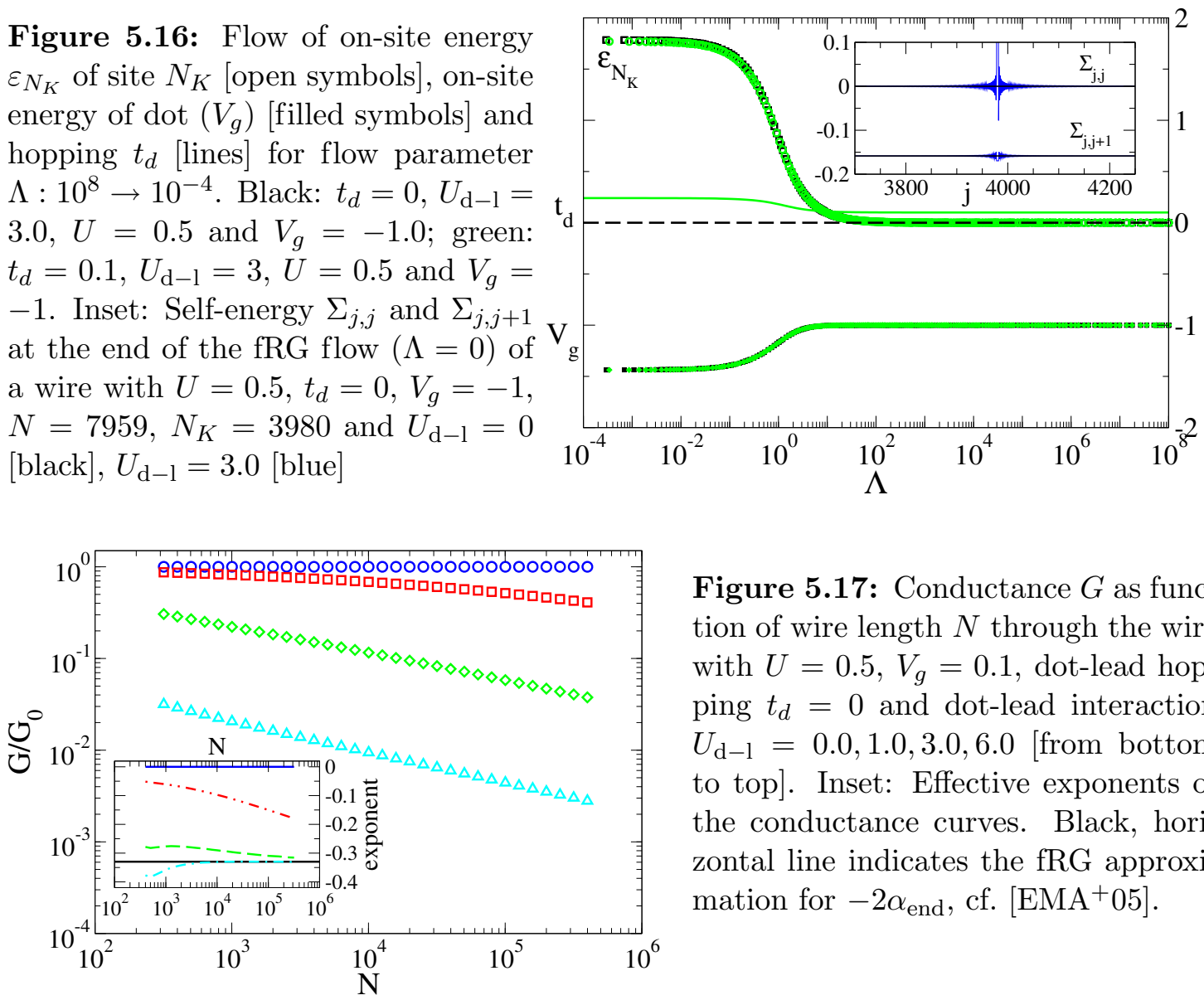

Figure 5.17: Conductance $G$ as function of wire length $N$ through the wire with $U=0.5, V_{g}=0.1$, dot-lead hopping $t_{d}=0$ and dot-lead interaction $U_{\mathrm{d}-1}=0.0,1.0,3.0,6.0$ [from bottom to top]. Inset: Effective exponents of the conductance curves. Black, horizontal line indicates the fRG approximation for $-2 \alpha_{\text {end }}$, cf. [EMA $\left.{ }^{+} 05\right]$.

$U_{\mathrm{d}-1} \neq 0$, the renormalized on-site-energy $\varepsilon_{N_{K}}$ induces oscillations in $\Sigma_{j, j}$ and $\Sigma_{j, j+1}$, which symmetrically decay with inverse distance around $N_{K}$. The oscillations are similar to those originating from a single site impurity, cf. section 4.1; interacting electrons and $\mathrm{AEM}^{+} 04$, YGM94.

Fig. 5.17 shows the scaling of the conductance $G$ with the system length $N$ for an interacting wire $(U=0.5)$, gate voltage $V_{g}=0.1$, zero dot-lead-hopping $t_{d}=0$ and various dot-lead interactions. For $U_{\mathrm{d}-1}=0$, the conductance stays perfect $\left(G / G_{0}=1\right)$ for any length $N$ as expected due to the perfect coupling of the wire to the leads. For $U_{\mathrm{d}-1}>0$, the conductance clearly exhibits power-law decay for large $N$. The exponent of the decay [cf. inset] asymptotically reaches a value of $-2 \alpha_{\text {end }}$, thus the conductance shows the scaling $G / G_{0} \sim N^{-2 \alpha_{\text {end }}}$, characteristic for a single site impurity embedded in a Luttinger liquid [KF92b, MMSS02b].

In summary, a detached $\left(t_{d}=0\right)$ dot with non-vanishing dot-lead interaction $\left(U_{\mathrm{d}-1}>0\right)$ has a similar effect on the self-energy and thereby the conductance as a single site impurity. The asymptotic low-energy behavior is described by the "openchain" fixed point KF92b. A similar scenario was found in the case of a single spin interacting locally with the spin density in an otherwise homogeneous Luttinger liquid (Kondo impurity in a Luttinger liquid) [FN94, Fur05, SI97]. 



\section{concluding remarks}

A short summary is given in this chapter and some future prospects are being discussed.

\section{\begin{tabular}{l|l} 
conductance of Luttinger-liquid wires & 6.1
\end{tabular}}

The first objective of this thesis was to generalize the studies on the conductance of a Luttinger-liquid wire by including the geometrical features necessarily appearing when coupling a finite wire to (higher-dimensional) leads. A major subobjective was the generalization of an existing scheme of the functional renormalization group to include these features.

In chapter 4, it was demonstrated that overhanging parts of the wire and leads induce energy scales, which lead to a split up of the basic temperature range $\pi v_{F} / L \ll T \ll B$ in which power-law scaling in the conductance can occur. The energy scales were shown to be inversely proportional to the length of the respective overhanging part. For a non-interacting wire $(K=1)$, the plateau of constant conductance in the range $\pi v_{F} / L \ll T \ll B$ splits into up to five plateaus of distinct constant conductance, separated by extended crossover regions. In going over to an interacting wire $(K<1)$, the energy scales remain unchanged, but the plateaus of formerly constant conductance show distinct power-law behavior $G \sim T^{\alpha(K)}$, still interrupted by extended crossover regimes. The scaling exponent $\alpha(K)$ was found to be $\alpha_{\text {end }}$ or $\alpha_{\text {bulk }}$, depending on wether the impact of the ends of the wire (boundary behavior) is readily cut off by the finite temperature. In temperature regimes, in which this impact was only cut off in one coupling region, no power law could be identified, however, scaling simply with $\alpha_{\text {end }}$ or $\alpha_{\text {bulk }}$ could be excluded. To further investigate this regime, an extended fRG scheme is needed, which includes the bulk exponent by e. g. keeping the frequency dependence of the two-particle vertex $\left[\mathrm{AEM}^{+} 04\right.$, p. 12]. All results were shown to hold for one-dimensional leads as well as for two-dimensional leads with extended coupling regions. Furthermore, the phenomena were argued to be robust against asymmetry of the width of the coupling regions and locally varying coupling strengths as long as the regime of low transmittance contacts is not being left.

Concerning experiments, which aim at the observation of power-law scaling in the temperature dependence of the conductance, the split up of the range $\pi v_{F} / L \ll$ $T \ll B$ into distinct regions of (potentially) distinct scaling behavior with extended separating crossover regions constitutes a significant difficulty. It indicates, that all lengths scales appearing in a specific experimental setup are potentially important 
and have to be treated with care. Setups have to be optimized in order to ensure that power-law behavior is not destroyed by the geometry of the setup in the accessible temperature range. One can speculate, that the inclusion of other phenomena, like inelastic scattering or relaxation and dissipation in the leads, which were neglected in this thesis, would worsen the situation by lowering the upper bound $B$ and leading to further obscuration of the power laws.

An interesting field for further development of the modeling and the fRG scheme of this thesis is the study of four-point measurements on Luttinger-liquid wires, cf. e. g. the theoretical studies [LRS02, DRS04, ANS08] and the experiments [YPBD99, $\mathrm{GKE}^{+} 04$. Steps into the direction of studying multi-terminal geometries within the fRG were done in [BTSMS05b, BTSMS05a, JMS07]. In particular, [JMS07] introduced a simple fRG scheme including a finite bias voltage, which, in combination with the modeling of extended leads and coupling regions presented in this thesis, would provide a unique tool for studying four-point measurements and resistances.

The role of spin was briefly discussed in the side note on page 49. The inclusion of spin- $\frac{1}{2}$ in the fRG scheme presented in section 3.1 is possible and some interesting studies on interacting quantum wires were performed And06, $\mathrm{AEM}^{+} 06 \mathrm{~b}, \mathrm{BM} 09$. A next step would be the adaption of the scheme developed in this thesis to spinfull systems. This would open the possibility to study further intriguing phenomena, like the appearance of the "0.7-structure" in the conductance, which is generally attributed to the interplay of spin and interaction ${ }^{1}$

\section{quantum-dot structures with Luttinger-liquid leads}

The second objective was to identify novel power laws in the observables of a single quantum dot, coupled to Luttinger-liquid leads and give hints on their utilization in an experimental setup, specifically suited to probe Luttinger-liquid predictions. A subobjective was to show, how the fRG scheme for treating complex coupling geometries can be adapted to study complex dot structures with Luttinger-liquid leads.

In chapter 5, the charging $n$ of a single-level quantum dot was studied in lowest order perturbation theory in the coupling to two (semi-infinite) Luttinger-liquid leads. Depending on the strength of the interaction in the leads (parametrized by the Luttinger-liquid parameter $K$ ), several regimes of distinct dependence on the gate voltage $V_{g}$ were identified. For $1 / 2<K \leq 1$, the charging is continuous. In the regime $1 / 2<K<2 / 3$ it approaches $1 / 2$ in leading order of $V_{g}$ in a power law with exponent $(2 K-1) /(1-K)$. For $0<K<1 / 2$, the charging has got a jump at $V_{g}=0$, which it approaches showing a power law with exponent $1 / K-2$ in the regime $1 / 3<K<1 / 2$. Furthermore, the characteristic width over which the continuous $n\left(V_{g}\right)$ changes was shown to exhibit a power-law dependence on the

\footnotetext{
${ }^{1}$ The literature in the field is vast, but the recent papers $\mathrm{KMHM}_{07}$, $\mathrm{DKC}^{+} 08$, $\mathrm{SGT}^{+} 08$ with their references provide an overview.
} 
coupling to the leads with exponent $K /(2 K-1)$. The results were complemented with an fRG calculation.

The power-law behavior of the charging was used to propose an experiment, in which the Luttinger-liquid parameter $K$ could be extracted from two observables as function of two distinct external parameters. This experiment circumvents the problem of theoretically estimating the parameter $K$ from first principles. Admittedly, an implementation of the scheme is a rather delicate experimental task, however, recent progress in the fabrication of highly tunable dots in carbon nanotubes [MBM04, $\mathrm{BGM}^{+}$05, JGRHL06, [SMB ${ }^{+}$06, SGK09] shows a promising way.

In a side note, recent studies on the charging of single-level quantum dots, sidecoupled or end-coupled to chiral and non-chiral Luttinger liquids were reviewed. In each model, certain regimes of the interaction strength (i. e. regimes of $K$ ) were identified, in which the charging approaches its value at vanishing gate voltage following characteristic power laws with exponent depending only on the Luttingerliquid parameter $K$. The fact that a single level, weakly end-coupled to a nonchiral Luttinger liquid can be shown to be equivalent to a single level side-coupled to a chiral Luttinger liquid [FM02 motivates the speculation that some similar equivalence might exist between a level, side-coupled to a non-chiral Luttinger liquid, a level, coupled to two chiral Luttinger liquids and (at least for weak coupling) a level coupled to two non-chiral Luttinger liquids. A first starting point might be the Coulomb gas GB09. Such an equivalence would provide a first step to perform an experiment as described in the preceding paragraph on quantum Hall bars [Cha03.

The fRG scheme was adapted to the study of complex dot geometries, cf. section 5.1, and its usefulness exemplified on the conductance and local spectral function of a parallel double quantum dot with Luttinger-liquid leads. This example can be extended by including a capacitive coupling $\hat{U}$ between the two dot levels, using the fRG schemes presented in [MM06, KEM06] to obtain flow equations for the dot levels and the interdot coupling. For Fermi liquid leads and tuned dots, the conductance as function of gate voltage was shown to exhibit resonances (so called "correlationinduced resonances") above some critical $\hat{U}_{c}$ [MM06]. Their position was identified as the Kondo temperature of an anisotropic Kondo model with magnetic field, thus getting exponentially smaller with increasing $\hat{U}>\hat{U}_{c}$ [KSAEW07]. The dependence of this position on the interaction in the leads, particularly changes in the functional form of its $\hat{U}$-dependence, is currently being studied. Traces of Luttinger-liquid physics are looked for by including interacting leads in the mappings of [KSAEW07] to the Kondo model.

Two interesting possible extensions of the fRG scheme shall finally be noted. First, interaction between the electrons on the dots and those in the leads could be included, cf. the side note on page 67 for a short example. To this end, the parametrization of the two-particle vertex, cf. section 3.1.2, has to be extended to include the renormalization of the respective more-than-nearest-neighbour hoppings appearing in the implementation of general dot structures, cf. section 5.1. Minimally this could be done by adding an appropriate $\Lambda$-independent term to (3.12). Second, 
the spin degree could be included, allowing for the study of the interplay of spin related phenomena in quantum $\operatorname{dot}^{2}$ and Luttinger-liquid physics in the leads.

${ }^{2}$ Compare with KEM06, $\mathrm{HKP}^{+} 07$ and references therein. 


\section{appendices}





\section{normalization of physical units}

A

The following conventions concerning the physical units are used throughout the thesis.

- Charge is measured in units of the elementary charge $e$.

- Temperature is measured in units of energy by setting $k_{B}=1$.

- Angular momentum is measured in units of action by setting $\hbar=1$.

In this convention, the conductance quantum $e^{2} / h=38.75 \mu \mathrm{S}$ is given as

$$
G_{0}=\frac{1}{2 \pi}
$$

Anytime the lattice model for the quantum wire (cf. section 2.2) is studied:

- energy is measured in units of the hopping $t$, cf. (2.3),

- distance is measured in units of the lattice spacing.

For example, temperatures $T$ can in this case be converted in SI units by $T[K]=$ $\frac{t}{k_{B}} T$. 



\section{projection formalism}

B

In the treatment of the semi-infinite leads throughout this work and in the algorithm described in section C.2 multiple use is made of the projection formula (so called "Feshbach projection")

$$
P \mathcal{G} P=\left(z P-P h P-P h Q(z Q-Q h Q)^{-1} Q h P\right)^{-1} .
$$

Therefor a short derivation is given following Feshbach's reformulation [Fes62] of his original work [Fes58].

Suppose $h$ being an Hermitian one-particle operator and define some projection operators $P$ and $Q=1-P$. The resolvent $\mathcal{G}=(z-h)^{-1}$ fulfills by definition

$$
(z-h) \mathcal{G}=1
$$

Operating on this equation with $P$ respectively $Q$ from the left and $P$ from the right the equations

$$
\begin{aligned}
& (z P-P h P) P \mathcal{G} P-P h Q Q \mathcal{G} P=P \\
& (z Q-Q h Q) Q \mathcal{G} P-Q h P P \mathcal{G} P=0
\end{aligned}
$$

are obtained. Note the insertion of $1=P+Q$ in front of $\mathcal{G}$. The second equation gives

$$
Q \mathcal{G} P=(z Q-Q h Q)^{-1} Q h P P \mathcal{G} P
$$

and upon inserting this in the first equation

$$
\left(z P-P h P-P h Q(z Q-Q h Q)^{-1} Q h P\right) P \mathcal{G} P=P \quad,
$$

which is equivalent to B.1. Similarly one obtains

$$
P \mathcal{G} Q=P \mathcal{G P} P Q(z Q-Q h Q)^{-1}
$$

and

$$
Q \mathcal{G} Q=(z Q-Q h Q)^{-1}+(z Q-Q h Q)^{-1} Q h P P \mathcal{G P P h} Q(z Q-Q h Q)^{-1}
$$

which are presented for later reference. 



\section{the inversion algorithms}

In this appendix, the algorithms needed to efficiently compute the Green functions in the flow equations (3.14a), (3.14b) and the transmission probabilities (E.4) are derived, cf. also chapter 4 and 5. The inversion algorithm for tridiagonal matrices (subsection C.1) was developed in $\left[\mathrm{AEM}^{+} 04\right]$. As this algorithm is build upon in the algorithm for complex geometries (subsection C.2), it is re-derived here in an elementary fashion.

\section{tridiagonal matrices $\mid$ C.1}

The following algorithm calculates the subdiagonal, superdiagonal and diagonal elements of the inverse of a symmetric, tridiagonal matrix.

Suppose A being a complex, symmetric, tridiagonal $(N \times N)$-matrix. The first step is to decompose $\mathbf{A}$ in a lower and upper triangular matrix $\mathbf{L}$ and $\mathbf{U}$, with $\mathbf{L}$ having 1 on the diagonal. The elements in the defining equation

$$
\mathbf{A}=\left(\begin{array}{lllll}
a_{1} & b_{1} & & & \\
b_{1} & a_{2} & b_{2} & & \\
& b_{2} & a_{3} & b_{3} & \\
& & \ddots & \ddots & \ddots
\end{array}\right)=\left(\begin{array}{ccccc}
1 & & & \\
l_{1} & 1 & & \\
& l_{2} & 1 & \\
& & \ddots & \ddots
\end{array}\right)\left(\begin{array}{llll}
m_{1} & r_{1} & & \\
& m_{2} & r_{2} & \\
& & \ddots & \ddots
\end{array}\right)=\mathbf{L} \mathbf{U}
$$

are calculated using a standard algorithm [SK04, p.62]:

$$
\begin{gathered}
m_{1}=a_{1} \\
\text { with } i=1,2, \ldots, N-1: \\
l_{i}=\frac{b_{i}}{m_{i}} \\
m_{i+1}=a_{i+1}-\frac{b_{i}^{2}}{m_{i}} .
\end{gathered}
$$

Define $\mathbf{Z}$ as the inverse of $\mathbf{A}$, i. e. $\mathbf{A Z}=\mathbf{L} \mathbf{U Z}=\mathbf{1}$ or

$$
\mathbf{U Z}=\mathbf{L}^{-1}
$$


with $\mathbf{L}^{-1}$ again being lower tridiagonal with 1 on the diagonal. In components, the equation reads

$$
\left(\begin{array}{cccc}
m_{1} & r_{1} & & \\
& m_{2} & r_{2} & \\
& & \ddots & \ddots
\end{array}\right)\left(\begin{array}{ccccc}
z_{11} & z_{12} & & & \\
z_{12} & z_{22} & z_{23} & & \bullet \\
& \ddots & \ddots & \ddots & \\
& \bullet & \ddots & \ddots & \ddots
\end{array}\right)=\left(\begin{array}{cccc}
1 & & & \\
& 1 & & \\
& & \ddots & \\
& \bullet & & \ddots
\end{array}\right) .
$$

The symbol • represents all elements off the subdiagonal, superdiagonal and diagonal, which are neither needed nor calculated. By explicitly writing down the set of linear equations for the $z_{i j}$ starting at the last row, one ends up with the following algorithm:

$$
\begin{gathered}
z_{N N}=m_{n}^{-1} \\
\text { with } i=N-1, N-2, \ldots, 1: \\
z_{i i+1}=-\frac{r_{i} z_{i+1 i+1}}{m_{i}} \\
z_{i i}=m_{i}^{-1}\left(1-r_{i} z_{i i+1}\right) .
\end{gathered}
$$

Starting from the $L R$-decomposition, the calculation of any single element of the inverse of $\mathbf{A}$ can be done using standard forward and backward substitution [SK04, p. 62 et seq.].

The runtime of the $L R$-decomposition, the calculation of the tridiagonal part of the inverse and the substitutions scale linearly with the dimension $N$ of the matrix A.

\section{algorithm for complex geometries}

Suppose $\mathcal{G}(z)=(z-h)^{-1}$ being the resolvent of an Hermitian operator $h$, whose representation as a $(N \times N)$-matrix consists of (large) tridiagonal blocks and (small) full blocks, connected by elements on the sub- and superdiagonal, cf. fig. C.1. The blocks on the diagonal are numbered from 1 to 5, while the off-diagonal blocks are numbered relative to the diagonal blocks. For example, the block $(1,3)$ refers to the elements $\langle i|\mathcal{G}| j\rangle$ with $i \in\left\{1, \ldots, N_{1}\right\}$ and $j \in\left\{N_{2}+1, \ldots, N_{3}\right\}$. Note that in the representation of $h$ all such off-diagonal blocks merely contain zeros.

The first of the following algorithms calculates efficiently the subdiagonal, superdiagonal and diagonal elements of $\mathcal{G}(z)$, which are needed in the calculation of the flow equations (3.14a) and (3.14b). Efficiently means, that the problem is decomposed such that only matrices of size and structure of the isolated blocks 1 to 5 have to be inverted. Accordingly, the (large) tridiagonal parts can be treated with the algorithm of subsection C.1, while a standard algorithm, e. g. [PTVF07, p. 54], has only to be used for the (small) full parts. This allows for the treatment of matrices up to $N \approx 10^{6}$ on an standard desktop PC. A short version of the derivation was already published in WMS09. 


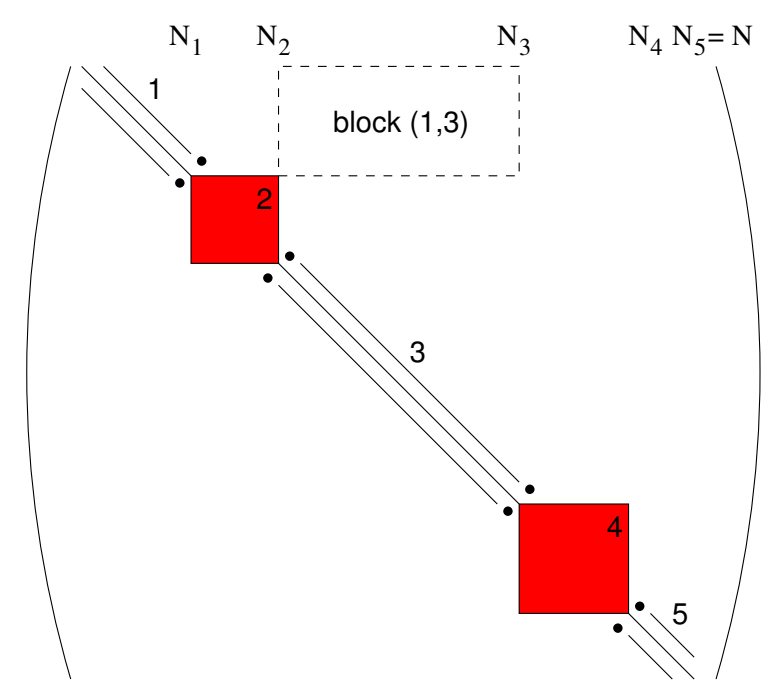

Figure C.1: Structure of the matrix: block 1, 3 and 5 are tridiagonal, block 2 and 4 are full matrices; the dots represent the connecting elements $\left(N_{j}, N_{j}\right)$ is the lower right element of the j-th block. The dashed rectangle exemplifies the numbering of the offdiagonal blocks.

The second algorithm calculates efficiently the block $(2,4)$ of $\mathcal{G}$, i. e. all $\langle i|\mathcal{G}| j\rangle$ with $i \in\left\{N_{1}+1, \ldots, N_{2}\right\}$ and $j \in\left\{N_{3}+1, \ldots, N_{4}\right\}$, which is needed in the calculation of the transmission probability (E.4). Efficiently again means, that the problem is decomposed such that only matrices of size and structure of the isolated blocks 1 to 5 have to be inverted, which again allows the usage of the algorithm in subsection C.1.

Note that both algorithms trivially work as well for a matrix, which has only one full block, i. e. one which is cut after block 3, cf. fig. C.1.

\section{subdiagonal, superdiagonal and diagonal}

Remember the basic definition $\mathcal{G}(z)=(z-h)^{-1}$ from above. The first step is to split $h$ into five smaller parts $h_{j}$, referring to block $j \in\{1, \ldots, 5\}$ respectively and elements $h_{j, j+1}^{\text {con }}=\beta_{j, j+1}\left|N_{j}\right\rangle\left\langle N_{j}+1\right|+$ H.c. connecting block $j$ and $j+1$ with $j \in\{1, \ldots, 4\}$ and $\beta_{j, j+1} \in \mathbb{R}$. Explicitly

$$
h=\sum_{j=1}^{5} h_{j}+\sum_{j=1}^{4} h_{j, j+1}^{\mathrm{con}} .
$$

Next define the projection operators $P_{j}$ on each block. The basic reasoning is to project the full resolvent $\mathcal{G}$ onto the block, whose elements are to be computed, thereby reducing the effort of inverting the full matrix to the inversion of an effective matrix of size and structure of block $j$ of $\mathcal{G}$. Note that the projection does not include 
any approximations. The following calculation of the elements of blocks 1 and 2 as well as those elements on the sub- and superdiagonal connecting block 1 and 2 exemplifies this reasoning. Making subsequent use of the projection formula (B.1),

$$
\begin{aligned}
P_{1} \mathcal{G} P_{1} & =\left(z-h_{1}-\beta_{1,2}^{2}\left|N_{1}\right\rangle\left\langle N_{1}+1\left|\tilde{\mathcal{G}}^{(2)}\right| N_{1}+1\right\rangle\left\langle N_{1}\right|\right)^{-1}, \\
P_{2} \tilde{\mathcal{G}}^{(2)} P_{2} & =\left(z-h_{2}-\beta_{2,3}^{2}\left|N_{2}\right\rangle\left\langle N_{2}+1\left|\tilde{\mathcal{G}}^{(3)}\right| N_{2}+1\right\rangle\left\langle N_{2}\right|\right)^{-1}, \\
P_{3} \tilde{\mathcal{G}}^{(3)} P_{3} & =\left(z-h_{3}-\beta_{3,4}^{2}\left|N_{3}\right\rangle\left\langle N_{3}+1\left|\tilde{\mathcal{G}}^{(4)}\right| N_{3}+1\right\rangle\left\langle N_{3}\right|\right)^{-1}, \\
P_{4} \tilde{\mathcal{G}}^{(4)} P_{4} & =\left(z-h_{4}-\beta_{4,5}^{2}\left|N_{4}\right\rangle\left\langle N_{4}+1\left|\tilde{\mathcal{G}}^{(5)}\right| N_{4}+1\right\rangle\left\langle N_{4}\right|\right)^{-1}, \\
P_{5} \tilde{\mathcal{G}}^{(5)} P_{5} & =
\end{aligned}
$$

with $\tilde{\mathcal{G}}^{(i)}=\left(z-\sum_{j=i}^{5} h_{j}-\sum_{j=i}^{4} h_{j, j+1}^{\text {con }}\right)^{-1}$ is obtained. The elements of block 1 of the resolvent $\mathcal{G}$ are now calculated by going through this hierarchy from bottom to top, necessitating only the inversion of matrices of size and structure of the isolated blocks at each step. Similarly, the elements of block 2 can be calculated going through the hierarchy

$$
\begin{aligned}
& P_{1} \tilde{\tilde{\mathcal{G}}}^{(1)} P_{1}=\left(z-h_{1}\right)^{-1}, \\
& P_{2} \mathcal{G} P_{2}= \\
& \left(z-h_{2}-\beta_{1,2}^{2}\left|N_{1}\right\rangle\left\langle N_{1}+1\left|\tilde{\tilde{\mathcal{G}}}^{(1)}\right| N_{1}+1\right\rangle\left\langle N_{1}\left|-\beta_{2,3}^{2}\right| N_{2}\right\rangle\left\langle N_{2}+1\left|\tilde{\mathcal{G}}^{(3)}\right| N_{2}+1\right\rangle\left\langle N_{2}\right|\right)^{-1}, \\
& P_{3} \tilde{\mathcal{G}}^{(3)} P_{3}=\left(z-h_{3}-\beta_{3,4}^{2}\left|N_{3}\right\rangle\left\langle N_{3}+1\left|\tilde{\mathcal{G}}^{(4)}\right| N_{3}+1\right\rangle\left\langle N_{3}\right|\right)^{-1}, \\
& P_{4} \tilde{\mathcal{G}}^{(4)} P_{4}=\left(z-h_{4}-\beta_{4,5}^{2}\left|N_{4}\right\rangle\left\langle N_{4}+1\left|\tilde{\mathcal{G}}^{(5)}\right| N_{4}+1\right\rangle\left\langle N_{4}\right|\right)^{-1}, \\
& P_{5} \tilde{\mathcal{G}}^{(5)} P_{5}=\left(z-h_{5}\right)^{-1}
\end{aligned}
$$

with $\tilde{\tilde{\mathcal{G}}}^{(i)}=\left(z-\sum_{j=1}^{i} h_{j}-\sum_{j=2}^{i} h_{j-1, j}^{\text {con }}\right)^{-1}$ beginning from top and bottom. The computations for the other blocks of $\mathcal{G}$ can be performed analogously.

The connecting element $\left\langle N_{1}+1|\mathcal{G}| N_{1}\right\rangle$ can be calculated by splitting $h$ into $\tilde{h}=\sum_{j=1}^{5} h_{j}+\sum_{j=2}^{4} h_{j, j+1}^{\text {con }}$ and $h_{1,2}^{\text {con }}$ to write

$$
\mathcal{G}=\tilde{\mathcal{G}}+\tilde{\mathcal{G}} h_{1,2}^{\text {con }} \tilde{\mathcal{G}}+\tilde{\mathcal{G}} h_{1,2}^{\text {con }} \mathcal{G} h_{1,2}^{\text {con }} \tilde{\mathcal{G}}
$$

with $\tilde{\mathcal{G}}=(z-\tilde{h})^{-1}$. After projecting $\mathcal{G}$ onto the blocks of interest, $P_{1} \mathcal{G} P_{2}$ and $P_{2} \mathcal{G} P_{1}$ and calculating the elements $\left\langle N_{1}+1|\mathcal{G}| N_{1}\right\rangle$ and $\left\langle N_{1}+1|\mathcal{G}| N_{1}\right\rangle$ respectively one ends up with a system of two linear equation, whose solution is

$$
\begin{aligned}
\left\langle N_{1}+1|\mathcal{G}| N_{1}\right\rangle= & \frac{\beta_{1,2}\left\langle N_{1}|\tilde{\mathcal{G}}| N_{1}\right\rangle\left\langle N_{1}+1|\tilde{\mathcal{G}}| N_{1}+1\right\rangle}{1-\beta_{1,2}^{4}\left\langle N_{1}|\tilde{\mathcal{G}}| N_{1}\right\rangle^{2}\left\langle N_{1}+1|\tilde{\mathcal{G}}| N_{1}+1\right\rangle^{2}} \\
& \times\left(1+\beta_{1,2}^{2}\left\langle N_{1}|\tilde{\mathcal{G}}| N_{1}\right\rangle\left\langle N_{1}+1|\tilde{\mathcal{G}}| N_{1}+1\right\rangle\right)
\end{aligned}
$$




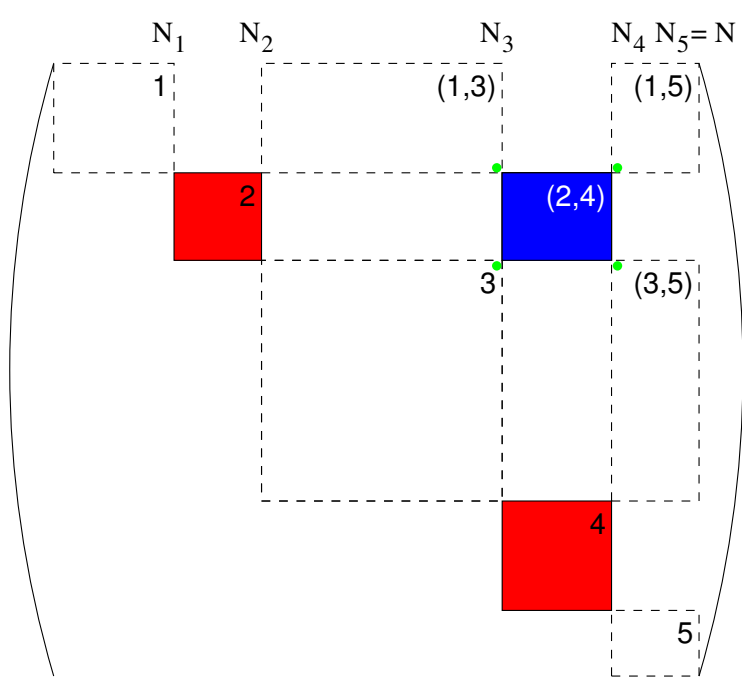

Figure C.2: Labeling of the blocks of $\mathcal{G}$ [for the structure of $\mathcal{G}^{-1}$ cf. fig. C.1]. The blue square [block $(2,4)]$ is to be calculated, the green dots represent $\left\langle N_{1}|\mathcal{G}| N_{3}\right\rangle,\left\langle N_{1}|\mathcal{G}| N_{4}+1\right\rangle$, $\left\langle N_{2}+1|\mathcal{G}| N_{4}+1\right\rangle$ and $\left\langle N_{2}+1|\mathcal{G}| N_{3}\right\rangle$ respectively.

The relevant elements of $\tilde{\mathcal{G}}$ can be efficiently calculated using the algorithm introduced above for the blocks. Again the calculation of the other elements $\left\langle N_{j}+1|\mathcal{G}| N_{j}\right\rangle$ can be performed analogously.

Note, that any single element from the tridiagonal blocks can be computed efficiently using this algorithm, cf. the comment at the end of section C.1.

\section{the block $(2,4)$}

In the calculation of the block $(2,4)$ of $\mathcal{G}$, i. e. the elements $\langle i|\mathcal{G}| j\rangle$ with $i \in\left\{N_{1}+\right.$ $\left.1, . ., N_{2}\right\}$ and $j \in\left\{N_{3}+1, \ldots, N_{4}\right\}$ (cf. fig. C.2 ), the first step is to split the full Hamiltonian C.2 into blocks $\tilde{h}=\sum_{j=1}^{5} h_{j}$ and connecting elements $h^{\text {con }}=\sum_{j=1}^{4} h_{j, j+1}^{\text {con }}$ to decompose

$$
\mathcal{G}=\tilde{\mathcal{G}}+\tilde{\mathcal{G}} h^{\mathrm{con}} \tilde{\mathcal{G}}+\tilde{\mathcal{G}} h^{\mathrm{con}} \mathcal{G} h^{\mathrm{con}} \tilde{\mathcal{G}}
$$

with $\tilde{\mathcal{G}}=(z-\tilde{h})^{-1}$. Multiplication with projection operators $P_{2}$ and $P_{4}$ yields

$$
\begin{aligned}
P_{2} \mathcal{G} P_{4}= & P_{2} \tilde{\mathcal{G}}\left(\beta_{2,3}\left|N_{2}\right\rangle\left\langle N_{2}+1\left|+\beta_{1,2}\right| N_{1}+1\right\rangle\left\langle N_{1}\right|\right) \\
& \mathcal{G}\left(\beta_{3,4}\left|N_{3}\right\rangle\left\langle N_{3}+1\left|+\beta_{4,5}\right| N_{4}+1\right\rangle\left\langle N_{4}\right|\right) \tilde{\mathcal{G}} P_{4}
\end{aligned}
$$

The elements of $\tilde{\mathcal{G}}$ can be efficiently computed as the disconnected blocks of $\tilde{\mathcal{G}}^{-1}$ can be inverted individually. The four elements $\left\langle N_{1}|\mathcal{G}| N_{3}\right\rangle,\left\langle N_{1}|\mathcal{G}| N_{4}+1\right\rangle,\left\langle N_{2}+\right.$ $\left.1|\mathcal{G}| N_{4}+1\right\rangle$ and $\left\langle N_{2}+1|\mathcal{G}| N_{3}\right\rangle$ constitute the corners at which the blocks $(1,3)$, $(1,5),(3,5)$ and 3 touch block $(2,4)$, cf. the green dots in fig. C.2. The further procedure relies on two basic notions. Firstly, elements lying inside the blocks 1 
to 5 can be computed efficiently using the algorithm described in subsection C.2.1. Secondly, when projecting on any block using the decomposition (C.3), only the corner elements of the blocks of $\mathcal{G}$ touching the block projected on at a single corner and some elements of $\tilde{\mathcal{G}}$ appear in the respective version of (C.4). This fact originates in the block structure of $\tilde{\mathcal{G}}$.

Thus, the element $\left\langle N_{2}+1|\mathcal{G}| N_{3}\right\rangle$ can be computed efficiently. The remaining three elements are calculated by projecting onto the respective blocks, cf. fig. C.2, leading to equations similar to (C.4) involving only corner elements from the blocks 2 and 4 and the block $(2,4)$ of $\mathcal{G}$ and elements of $\tilde{\mathcal{G}}$ as noted above. As an example, consider $\left\langle N_{1}|\mathcal{G}| N_{3}\right\rangle$. Projecting $\mathcal{G}$ onto the block (1,3) using (C.3) gives

$$
P_{1} \mathcal{G} P_{3}=P_{1} \tilde{\mathcal{G}}\left(\beta_{1,2}\left|N_{1}\right\rangle\left\langle N_{1}+1\right|\right) \mathcal{G}\left(\beta_{2,3}\left|N_{2}\right\rangle\left\langle N_{2}+1\left|+\beta_{3,4}\right| N_{3}+1\right\rangle\left\langle N_{3}\right|\right) \tilde{\mathcal{G}} P_{3}
$$

and thus

$$
\begin{array}{r}
\left\langle N_{1}|\mathcal{G}| N_{3}\right\rangle=\beta_{1,2}\left\langle N_{1}|\tilde{\mathcal{G}}| N_{1}\right\rangle\left(\beta_{2,3}\left\langle N_{1}+1|\mathcal{G}| N_{2}\right\rangle\left\langle N_{2}+1|\tilde{\mathcal{G}}| N_{3}\right\rangle\right. \\
\left.+\beta_{3,4}\left\langle N_{1}+1|\mathcal{G}| N_{3}+1\right\rangle\left\langle N_{3}|\tilde{\mathcal{G}}| N_{3}\right\rangle\right) .
\end{array}
$$

The element $\left\langle N_{1}+1|\mathcal{G}| N_{2}\right\rangle$ is the upper right corner of block 2 and can therefore be computed efficiently. $\left\langle N_{1}+1|\mathcal{G}| N_{3}+1\right\rangle$ constitutes the upper left corner of block $(2,4)$, thus can be evaluated using (C.4). By performing this projection for each element $\left\langle N_{1}|\mathcal{G}| N_{3}\right\rangle,\left\langle N_{1}|\mathcal{G}| N_{4}+1\right\rangle,\left\langle N_{2}+1|\mathcal{G}| N_{4}+1\right\rangle$ one obtains a closed set of equations, whose solution is given by

$$
\begin{aligned}
&\left\langle N_{1}|\mathcal{G}| N_{3}\right\rangle=-( D_{2}\left(D_{3}\left(A_{1}+A_{3}\right)+B_{1}\left(A_{2}+A_{5}\right)\right)+B_{5}\left(B_{4}\left(A_{2}+A_{5}\right)\right. \\
&-\left.\left.B_{6}\left(A_{1}+A_{3}\right)\right)+A_{4}\left(B_{4} D_{3}+B_{1} B_{6}\right)\right) \\
& /\left(D_{1}\left(B_{6} B_{5}-D_{2} D_{3}\right)+B_{2}\left(B_{4} D_{3}+B_{1} B_{6}\right)+B_{3}\left(B_{1} D_{2}+B_{4} B_{5}\right)\right) \\
&\left\langle N_{2}+1|\mathcal{G}| N_{4}+1\right\rangle=\left(D_{2}\left(A_{2}+A_{5}\right)+A_{4} B_{6}+\left(B_{3} D_{2}+B_{2} B_{6}\right)\left\langle N_{1}|\mathcal{G}| N_{3}\right\rangle\right) \\
& /\left(D_{2} D_{3}-B_{6} B_{5}\right) \\
&\left\langle N_{1}|\mathcal{G}| N_{4}+1\right\rangle=\left(A_{4}+B_{2}\left\langle N_{1}|\mathcal{G}| N_{3}\right\rangle+B_{5}\left\langle N_{2}+1|\mathcal{G}| N_{4}+1\right\rangle\right) / D_{3}
\end{aligned}
$$

with the abbreviations

$$
\begin{aligned}
& A_{1}=\beta_{1,2} \beta_{2,3}\left\langle N_{1}|\tilde{\mathcal{G}}| N_{1}\right\rangle\left\langle N_{1}+1|\mathcal{G}| N_{2}\right\rangle\left\langle N_{2}+1|\tilde{\mathcal{G}}| N_{3}\right\rangle \\
& A_{2}=\beta_{3,4} \beta_{4,5}\left\langle N_{2}+1|\tilde{\mathcal{G}}| N_{3}\right\rangle\left\langle N_{3}+1|\mathcal{G}| N_{4}\right\rangle\left\langle N_{4}+1|\tilde{\mathcal{G}}| N_{4}+1\right\rangle \\
& A_{3}=\beta_{1,2} \beta_{2,3} \beta_{3,4}^{2}\left\langle N_{1}|\tilde{\mathcal{G}}| N_{1}\right\rangle\left\langle N_{1}+1|\tilde{\mathcal{G}}| N_{2}\right\rangle\left\langle N_{2}+1|\mathcal{G}| N_{3}\right\rangle\left\langle N_{3}+1|\tilde{\mathcal{G}}| N_{3}+1\right\rangle\left\langle N_{3}|\tilde{\mathcal{G}}| N_{3}\right\rangle \\
& A_{4}=\beta_{1,2} \beta_{2,3} \beta_{3,4} \beta_{4,5}\left\langle N_{1}|\tilde{\mathcal{G}}| N_{1}\right\rangle\left\langle N_{1}+1|\tilde{\mathcal{G}}| N_{2}\right\rangle\left\langle N_{2}+1|\mathcal{G}| N_{3}\right\rangle\left\langle N_{3}+1|\tilde{\mathcal{G}}| N_{4}\right\rangle\left\langle N_{4}+1|\tilde{\mathcal{G}}| N_{4}+1\right\rangle \\
& A_{5}=\beta_{2,3}^{2} \beta_{3,4} \beta_{4,5}\left\langle N_{2}+1|\tilde{\mathcal{G}}| N_{2}+1\right\rangle\left\langle N_{2}|\tilde{\mathcal{G}}| N_{2}\right\rangle\left\langle N_{2}+1|\mathcal{G}| N_{3}\right\rangle\left\langle N_{3}+1|\tilde{\mathcal{G}}| N_{4}\right\rangle\left\langle N_{4}+1|\tilde{\mathcal{G}}| N_{4}+1\right\rangle \\
& B_{1}=\beta_{1,2} \beta_{2,3} \beta_{3,4} \beta_{4,5}\left\langle N_{1}|\tilde{\mathcal{G}}| N_{1}\right\rangle\left\langle N_{1}+1|\tilde{\mathcal{G}}| N_{2}\right\rangle\left\langle N_{4}|\tilde{\mathcal{G}}| N_{3}+1\right\rangle\left\langle N_{3}|\tilde{\mathcal{G}}| N_{3}\right\rangle \\
& B_{2}=\beta_{1,2}^{2} \beta_{3,4} \beta_{4,5}\left\langle N_{1}|\tilde{\mathcal{G}}| N_{1}\right\rangle\left\langle N_{1}+1|\tilde{\mathcal{G}}| N_{1}+1\right\rangle\left\langle N_{3}+1|\tilde{\mathcal{G}}| N_{4}\right\rangle\left\langle N_{4}+1|\tilde{\mathcal{G}}| N_{4}+1\right\rangle
\end{aligned}
$$




$$
\begin{aligned}
& B_{3}=\beta_{1,2} \beta_{2,3} \beta_{3,4} \beta_{4,5}\left\langle N_{2}+1|\tilde{\mathcal{G}}| N_{2}+1\right\rangle\left\langle N_{2}|\tilde{\mathcal{G}}| N_{1}+1\right\rangle\left\langle N_{3}+1|\tilde{\mathcal{G}}| N_{4}\right\rangle\left\langle N_{4}+1|\tilde{\mathcal{G}}| N_{4}+1\right\rangle \\
& B_{4}=\beta_{1,2}^{2} \beta_{3,4} \beta_{4,5}\left\langle N_{1}|\tilde{\mathcal{G}}| N_{1}\right\rangle\left\langle N_{1}+1|\tilde{\mathcal{G}}| N_{1}+1\right\rangle\left\langle N_{4}|\tilde{\mathcal{G}}| N_{3}+1\right\rangle\left\langle N_{3}|\tilde{\mathcal{G}}| N_{3}\right\rangle \\
& B_{5}=\beta_{1,2} \beta_{2,3} \beta_{3,4}^{2}\left\langle N_{1}|\tilde{\mathcal{G}}| N_{1}\right\rangle\left\langle N_{1}+1|\tilde{\mathcal{G}}| N_{2}\right\rangle\left\langle N_{4}|\tilde{\mathcal{G}}| N_{4}\right\rangle\left\langle N_{4}+1|\tilde{\mathcal{G}}| N_{4}+1\right\rangle \\
& B_{6}=\beta_{1,2} \beta_{2,3} \beta_{4,5}^{2}\left\langle N_{2}+1|\tilde{\mathcal{G}}| N_{2}+1\right\rangle\left\langle N_{2}|\tilde{\mathcal{G}}| N_{1}+1\right\rangle\left\langle N_{4}|\tilde{\mathcal{G}}| N_{4}\right\rangle\left\langle N_{4}+1|\tilde{\mathcal{G}}| N_{4}+1\right\rangle \\
& D_{1}=1-\beta_{1,2}^{2} \beta_{3,4}^{2}\left\langle N_{1}|\tilde{\mathcal{G}}| N_{1}\right\rangle\left\langle N_{1}+1|\tilde{\mathcal{G}}| N_{1}+1\right\rangle\left\langle N_{3}|\tilde{\mathcal{G}}| N_{3}\right\rangle\left\langle N_{3}+1|\tilde{\mathcal{G}}| N_{3}+1\right\rangle \\
& D_{2}=1-\beta_{1,2}^{2} \beta_{4,5}^{2}\left\langle N_{1}|\tilde{\mathcal{G}}| N_{1}\right\rangle\left\langle N_{1}+1|\tilde{\mathcal{G}}| N_{1}+1\right\rangle\left\langle N_{4}|\tilde{\mathcal{G}}| N_{4}\right\rangle\left\langle N_{4}+1|\tilde{\mathcal{G}}| N_{4}+1\right\rangle \\
& D_{3}=1-\beta_{2,3}^{2} \beta_{4,5}^{2}\left\langle N_{2}|\tilde{\mathcal{G}}| N_{2}\right\rangle\left\langle N_{2}+1|\tilde{\mathcal{G}}| N_{2}+1\right\rangle\left\langle N_{4}|\tilde{\mathcal{G}}| N_{4}\right\rangle\left\langle N_{4}+1|\tilde{\mathcal{G}}| N_{4}+1\right\rangle .
\end{aligned}
$$

With these equations, the calculation of $\left\langle N_{2}+1|\mathcal{G}| N_{3}\right\rangle,\left\langle N_{1}|\mathcal{G}| N_{3}\right\rangle,\left\langle N_{1}|\mathcal{G}| N_{4}+1\right\rangle$ and $\left\langle N_{2}+1|\mathcal{G}| N_{4}+1\right\rangle$ and therefore the calculation of any element of block $(2,4)$ by virtue of (C.4) only necessitates the computation of elements of $\tilde{\mathcal{G}}$ and elements from the blocks 1 to 5 of $\mathcal{G}$. All this can be done using the efficient algorithm described in subsection C.2.1. 



\section{the models for the leads}

$\mathrm{D}$

In this appendix a detailed technical account of the models for the leads, (2.7) and (2.8) is given. In particular, the resolvents of the models are derived in the representation needed.
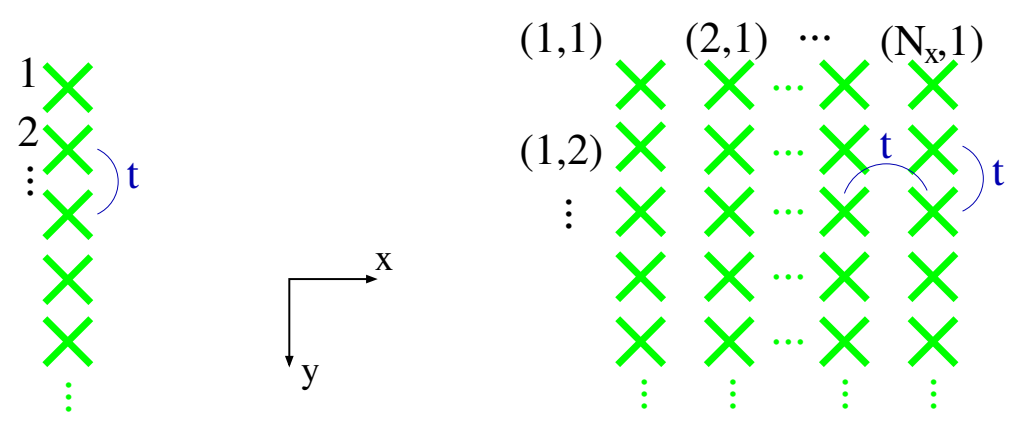

Figure D.1: Sketch of the one-dimensional hopping chain (left) and two-dimensional hopping stripe (right). Both are infinite in the $y$-direction; the numbers give the labeling scheme of the sites.

\section{\begin{tabular}{l|l} 
the semi-infinite hopping chain & D.1
\end{tabular}}

With the numbering introduced in fig. D.1, the Hamiltonian of the chain reads in Dirac notation

$$
h^{1 \mathrm{~d}}:=h^{1 \mathrm{~d}-\mathrm{lead}}=-t \sum_{j=1}^{\infty}(|j\rangle\langle j+1|+\text { H.c. })
$$

This is a discrete version of the problem of the particle in the box $\{1, \ldots, N\}$ in the limit $N \rightarrow \infty$ [CTDL77, vol. 1 p. 77 et seq.]. The eigenfunctions are thus plane waves, obeying the correct boundary condition:

$$
\langle j \mid k\rangle=\sqrt{\frac{2}{\pi}} \sin (k j)
$$

with $k \in(0, \pi)$. The dispersion relation is $\varepsilon(k)=-2 t \cos (k)$, the velocity AM76, p. 765] is defined as $v(k)=2 t \sin (k)$. 


\section{Green function of the one-dimensional chain}

D.2

Suppose $h^{1 \mathrm{~d}}$ to be the one-particle Hamiltonian of the semi-infinite chain and $\mathcal{G}^{1 \mathrm{~d}}=$ $\left(z-h^{1 \mathrm{~d}}\right)^{-1}$ its resolvent. Using the basic representation of $\mathcal{G}^{1 \mathrm{~d}}$ in the eigenbasis of $h^{1 \mathrm{~d}}$ (cf. [Eco79, p. 8]) one obtains

$$
\begin{aligned}
\left\langle j\left|\mathcal{G}^{1 \mathrm{~d}}\right| j^{\prime}\right\rangle & =\frac{2}{\pi} \int_{0}^{\pi} d k \frac{\sin (k j) \sin \left(k j^{\prime}\right)}{z+2 t \cos (k)} \\
& =\frac{1}{\pi} \int_{0}^{\pi} d k\left(\frac{\cos \left(k\left(j-j^{\prime}\right)\right)}{z+2 t \cos (k)}+\frac{\cos \left(k\left(j+j^{\prime}\right)\right)}{z+2 t \cos (k)}\right) .
\end{aligned}
$$

The identity $2 \sin (x) \sin (y)=\cos (x-y)-\cos (x+y)$ [AS72, p. 72] was used in the second line. The symmetry of the cosine further implies

$$
\frac{1}{\pi} \int_{0}^{\pi} d k \frac{\cos \left(k\left(j \pm j^{\prime}\right)\right)}{z+2 t \cos (k)}=\frac{1}{2 \pi} \int_{-\pi}^{\pi} d k \frac{e^{\imath k\left|j \pm j^{\prime}\right|}}{z+2 t \cos (k)} .
$$

As $2 \cos (x)=e^{\imath x}+e^{-\imath x}$, the integral only depends on $e^{\imath k}$ and can be solved by integrating over the unit circle in the complex plane, using the method of residues Eco79, p. 80 et seq.]:

$$
\frac{1}{2 \pi} \int_{-\pi}^{\pi} d k \frac{e^{\imath k\left|j \pm j^{\prime}\right|}}{z+2 t \cos (k)}=\frac{1}{\sqrt{z^{2}-4 t^{2}}}\left(-\frac{z}{2 t}+\frac{1}{2 t} \sqrt{z^{2}-4 t^{2}}\right)^{\left|j \pm j^{\prime}\right|} .
$$

Thus, the resolvent is given as

$$
\left\langle j\left|\mathcal{G}^{1 \mathrm{~d}}\right| j^{\prime}\right\rangle=\frac{1}{\sqrt{z^{2}-4 t^{2}}}\left(\left(-\frac{z}{2 t}+\frac{1}{2 t} \sqrt{z^{2}-4 t^{2}}\right)^{\left|j-j^{\prime}\right|}-\left(-\frac{z}{2 t}+\frac{1}{2 t} \sqrt{z^{2}-4 t^{2}}\right)^{\left|j+j^{\prime}\right|}\right) .
$$

Substituting $z \rightarrow \varepsilon+\imath \eta$ with $|\varepsilon|<2 t$ and $\eta \searrow 0$, this can be rewritten as

$$
\begin{aligned}
\left\langle j\left|\mathcal{G}^{1 \mathrm{~d}}(\varepsilon+\imath \eta)\right| j^{\prime}\right\rangle & =\frac{-\imath}{v[k(\varepsilon)]}\left(e^{\imath k(\varepsilon)\left|j-j^{\prime}\right|}-e^{\imath k(\varepsilon)\left|j+j^{\prime}\right|}\right) \\
& =-\frac{2 \sin \left(k(\varepsilon) j^{\prime}\right)}{v[k(\varepsilon)]} e^{\imath k(\varepsilon) j}
\end{aligned}
$$

whereat $k(\varepsilon)=\arccos \left(-\frac{\varepsilon}{2 t}\right)$ and $v(k)=2 t \sin (k)$. The second equality holds for $j \geq j^{\prime}$.

\section{the semi-infinite hopping stripe $\quad$ D.3}

With the basic definitions from fig. D.1, the Hamiltonian of the stripe reads

$$
h^{2 \mathrm{~d}}:=h^{2 \mathrm{~d}-\text { lead }}=-\sum_{\vec{\jmath}, \vec{\jmath}^{\prime} \in \mathbb{A}}\left(t^{\left(\vec{\jmath}, \vec{\jmath}^{\prime}\right)}|\vec{\jmath}\rangle\left\langle\vec{\jmath}^{\prime}\right|+\text { H.c. }\right)
$$


whereat $|\vec{\jmath}\rangle=\left|j_{x}, j_{y}\right\rangle=\left|j_{x}\right\rangle \otimes\left|j_{y}\right\rangle$ and $\mathbb{A}=\left\{\left(j_{x}, j_{y}\right)^{\mathrm{T}} \mid j_{x} \in\left\{1, \ldots, N_{x}\right\} \wedge j_{y} \in\right.$ $\{1, \ldots, \infty\}\}$. The matrix $t^{\left(\vec{\jmath}, \vec{\jmath}^{\prime}\right)}$ only connects nearest-neighbour sites. The eigenvalue problem is trivially separated [CTDL77, vol. 1 p. 60 et seq.], therefore the eigenvalues $\varepsilon(\vec{k})$ and eigenfunctions $\langle\vec{\jmath} \mid \vec{k}\rangle$ immediately follow from the one-dimensional case, cf. section D.1 as

$$
\begin{aligned}
\varepsilon(\vec{k}) & =-2 t_{x} \cos \left(k_{x}\right)-2 t_{y} \cos \left(k_{y}\right)=: \varepsilon_{x}\left(k_{x}\right)+\varepsilon_{y}\left(k_{y}\right) \quad \text { and } \\
\langle\vec{\jmath} \mid \vec{k}\rangle & =\sqrt{\frac{2}{N_{x}+1}} \sin \left(k_{x} j_{x}\right) \sqrt{\frac{2}{\pi}} \sin \left(k_{y} j_{y}\right) \quad,
\end{aligned}
$$

whereat $k_{y} \in(0, \pi)$ and $k_{x}=\frac{\pi}{N_{x}+1} n_{x}$ with $n_{x} \in\left\{1, \ldots, N_{x}\right\}$.

\section{Green function of the two-dimensional stripe}

Suppose $\mathcal{G}^{2 \mathrm{~d}}=\left(z-h^{2 \mathrm{~d}}\right)^{-1}$ to be the resolvent of (D.4). In the calculation of the transmission amplitude (E.4), its mixed representation, i. e. local in the $y$-direction and in $k$-space for the transverse direction, is needed:

$$
\begin{aligned}
\left\langle\tilde{k}_{x}, j_{y}\left|\mathcal{G}^{2 \mathrm{~d}}(z)\right| \vec{\jmath}^{\prime}\right\rangle & =\sum_{k_{x}} \frac{2}{\pi} \int_{0}^{\pi} d k_{y} \frac{\left\langle\tilde{k}_{x}, j_{y} \mid \vec{k}\right\rangle\left\langle\vec{k} \mid \vec{\jmath}^{\prime}\right\rangle}{z+2 t_{x} \cos \left(k_{x}\right)+2 t_{y} \cos \left(k_{y}\right)} \\
& =\left\langle\tilde{k}_{x} \mid j_{x}^{\prime}\right\rangle \frac{2}{\pi} \int_{0}^{\pi} d k_{y} \frac{\sin \left(k_{y} j_{y}\right) \sin \left(k_{y} j_{y}^{\prime}\right)}{z+2 t_{x} \cos \left(\tilde{k}_{x}\right)+2 t_{y} \cos \left(k_{y}\right)} \\
& =\left\langle\tilde{k}_{x} \mid j_{x}^{\prime}\right\rangle\left\langle j_{y}\left|\mathcal{G}^{1 \mathrm{~d}}(\tilde{z})\right| j_{y}^{\prime}\right\rangle
\end{aligned}
$$

by eq. (D.1) and defining $\tilde{z}=z+2 t_{x} \cos \left(\tilde{k}_{x}\right)$. The substitution $z \rightarrow \varepsilon\left(\tilde{k}_{x}, k_{y}\right)+\imath \eta$ with $\eta \searrow 0$, i. e. $\tilde{z} \rightarrow-2 t_{y} \cos \left(k_{y}\right)+\imath \eta$, gives with (D.3)

$$
\begin{aligned}
\left\langle\tilde{k}_{x}, j_{y}\left|\mathcal{G}^{2 \mathrm{~d}}(\varepsilon+\imath \eta)\right| \vec{\jmath}^{\prime}\right\rangle & =-\imath \frac{\left\langle\tilde{k}_{x} \mid j_{x}^{\prime}\right\rangle}{v_{y}\left[k_{y}(\varepsilon)\right]}\left(e^{\imath k_{y}(\varepsilon)\left|j_{y}-j_{y}^{\prime}\right|}-e^{\imath k_{y}(\varepsilon)\left|j_{y}+j_{y}^{\prime}\right|}\right) \\
& \stackrel{j_{y} \geq j_{y}^{\prime}}{=}-\frac{2\left\langle\tilde{k}_{x} \mid j_{x}^{\prime}\right\rangle \sin \left(k_{y}(\varepsilon) j_{y}^{\prime}\right)}{v_{y}\left[k_{y}(\varepsilon)\right]} e^{\imath k_{y}(\varepsilon) j_{y}}
\end{aligned}
$$

whereat $k_{y}(\varepsilon)=\arccos \left(-\frac{\varepsilon+2 t_{x} \cos \left(\tilde{k}_{x}\right)}{2 t_{y}}\right)$ and $v_{y}\left(k_{y}\right)=2 t_{y} \sin \left(k_{y}\right)$.

In a pure position space representation, the elements $\left\langle\vec{\jmath}\left|\mathcal{G}^{2 \mathrm{~d}}(z)\right| \vec{\jmath}^{\prime}\right\rangle$ read

$$
\left\langle\vec{\jmath}\left|\mathcal{G}^{2 \mathrm{~d}}(z)\right| \vec{\jmath}^{\prime}\right\rangle=\sum_{k_{x}}\left\langle j_{x} \mid k_{x}\right\rangle\left\langle k_{x} \mid j_{x}^{\prime}\right\rangle\left\langle j_{y}\left|\mathcal{G}^{1 \mathrm{~d}}(\tilde{z})\right| j_{y}^{\prime}\right\rangle
$$

Note that the summation cannot be done analytically, recently developed approaches, cf. Coj06 and Coj07, only hold for energies outside the band. In the limiting case $N_{x}=1$, i. e. $k_{x}=\pi / 2$, the one-dimensional Green function (D.3) is re-obtained. 



\section{explicit calculation of $\mathcal{T}^{(i)}$ and $\mathcal{T}^{(i i)} \quad \mathrm{E}$ \\ computing $\mathcal{T}^{(i)}$ : single-particle scattering theory $\quad$ E.1}

At the end of the approximate fRG flow, the one-particle Hamiltonian

$$
\bar{h}=h^{\text {wire }}+\Sigma+h_{L}^{\text {coupl }}+h_{R}^{\text {coupl }}+h_{L}^{\text {lead }}+h_{R}^{\text {lead }},
$$

is obtained, cf. (3.15). To compute the effective transmission probability $\mathcal{T}^{(i)}$ determining the conductance $G$ in the Landauer-Büttiker formula, cf. subsection 3.3.1. this Hamiltonian is decomposed into an unperturbed part $h_{0}:=h^{\text {wire }}+\Sigma+h_{L}^{\text {lead }}+h_{R}^{\text {lead }}$ and a perturbation $h_{1}:=h_{L}^{\text {coupl }}+h_{R}^{\text {coupl }}$ and the corresponding scattering problem is solved.

The outgoing scattering states $|\vec{k}+\rangle_{a}$ in lead $a$ obey the Lippmann-Schwinger equation [Sit71, p. 37]

$$
|\vec{k}+\rangle_{a}=|\vec{k}\rangle_{a}+\mathcal{G}\left(\varepsilon_{a}(\vec{k})+\imath \eta\right) h_{1}|\vec{k}\rangle_{a}
$$

with $\overline{\mathcal{G}}(z)=(z-\bar{h})^{-1}$ and $\eta \searrow 0$. The dispersion of lead $a$ is denoted by $\varepsilon_{a}(\vec{k})=$ $\varepsilon_{a, x}\left(k_{x}\right)+\varepsilon_{a, y}\left(k_{y}\right)$, its eigenstates by $|\vec{k}\rangle^{a}$, cf. appendix $\mathrm{D}$ for details. Inserting $h_{1}$ explicitly, cf. (2.9), the components in the representation, whose quantum numbers are the position $n_{y}$ in y-direction and $k_{x}$ are given by

$$
{ }_{\bar{a}}\left\langle k_{x}^{\prime}, n_{y} \mid \vec{k}+\right\rangle_{a}=\sum_{j=1}^{N} \sum_{\vec{l} \in \mathbb{A}_{a}}\left\langle k_{x}^{\prime}, n_{y}\left|\overline{\mathcal{G}}\left(\varepsilon_{a}(\vec{k})+\imath \eta\right)\right| j\right\rangle V_{a}^{(j, \vec{l})}{ }_{a}\langle\vec{l} \mid \vec{k}\rangle_{a}
$$

which, upon using the Dyson equation $\overline{\mathcal{G}}=\mathcal{G}_{0}+\mathcal{G}_{0} h_{1} \overline{\mathcal{G}}$ with $\mathcal{G}_{0}(z)=\left(z-h_{0}\right)^{-1}$, yields

$$
{ }_{\bar{a}}\left\langle k_{x}^{\prime}, n_{y} \mid \vec{k}+\right\rangle_{a}=\sum_{j, j^{\prime}=1}^{N} \sum_{\vec{l}^{\prime} \in \mathbb{A}_{\bar{a}} \vec{l} \in \mathbb{A}_{a}} \sum_{\bar{a}}\left\langle k_{x}^{\prime}, n_{y}\left|\mathcal{G}_{0}\right| \vec{l}^{\prime}\right\rangle_{\bar{a}} V_{\bar{a}}^{\left(\vec{l}^{\prime}, j^{\prime}\right)}\left\langle j^{\prime}|\overline{\mathcal{G}}| j\right\rangle V_{a}^{(j, \vec{l})}{ }_{a}\langle\vec{l} \mid \vec{k}\rangle_{a}
$$

The matrix element $\bar{a}_{\bar{a}}\left\langle k_{x}^{\prime}, n_{y}\left|\mathcal{G}_{0}\right| \vec{l}^{\prime}\right\rangle_{\bar{a}}$ was calculated in appendix D, eq. (D.5), and for $m_{y}>m_{y}^{\prime}$

${ }_{\bar{a}}\left\langle k_{x}^{\prime}, n_{y} \mid \vec{k}+\right\rangle_{a}=\left(-\sum_{j, j^{\prime}=1}^{N} \sum_{\vec{l}^{\prime} \in \mathbb{A}_{\bar{a}}} \sum_{\vec{l} \in \mathbb{A}_{a}} \frac{2 \pi}{v_{\bar{a}}\left(k_{y}^{\prime}\right)^{\bar{a}}} \overline{\langle} \vec{k}^{\prime}\left|\vec{l}^{\prime}\right\rangle_{\bar{a}} V_{\bar{a}}^{\left(\vec{l}^{\prime}, j^{\prime}\right)}\left\langle j^{\prime}|\overline{\mathcal{G}}| j\right\rangle V_{a}^{(j, \vec{l})}{ }_{a}\langle\vec{l} \mid \vec{k}\rangle_{a}\right) \frac{e^{2 k_{y}^{\prime} n_{y}}}{\sqrt{2 \pi}}$ 
is obtained upon insertion. For $\left|\varepsilon_{a}(\vec{k})-\varepsilon_{\bar{a}, x}\left(k_{x}^{\prime}\right)\right|<2\left|t_{\bar{a}, y}\right|, k_{y}^{\prime} \in(0, \pi)$ is determined by energy conservation $\varepsilon_{\bar{a}, y}\left(k_{y}^{\prime}\right)=\varepsilon_{a}(\vec{k})-\varepsilon_{\bar{a}, x}\left(k_{x}^{\prime}\right)$. For $\left|\varepsilon_{a}(\vec{k})-\varepsilon_{\bar{a}, x}\left(k_{x}^{\prime}\right)\right|>2\left|t_{\bar{a}, y}\right|$ the free resolvent $\mathcal{G}_{0}$ decays exponentially and thus "closes" the respective channels, such that only elastic scattering $\varepsilon_{a}(\vec{k})=\varepsilon_{\bar{a}}\left(\vec{k}^{\prime}\right)$ occurs.

The prefactor of the plane wave in $($ E.3 gives the transmission probability $\left|t_{|\vec{k}\rangle_{a} \rightarrow\left|\vec{k}^{\prime}\right\rangle_{\bar{a}}}\right|^{2}$ for the scattering event $|\vec{k}\rangle_{a} \rightarrow\left|\vec{k}^{\prime}\right\rangle_{\bar{a}}$ as

$$
\begin{aligned}
\left|t_{|\vec{k}\rangle_{a} \rightarrow\left|\vec{k}^{\prime}\right\rangle_{\bar{a}}}\right|^{2} & =\left|t\left(\varepsilon_{a}(\vec{k}), k_{x}^{\prime}\right)\right|^{2} \\
& =\left|\sum_{j, j^{\prime}=1}^{N} \sum_{\vec{l}^{\prime} \in \mathbb{A}_{\bar{a}}} \sum_{\vec{l} \in \mathbb{A}_{a}} \frac{2 \pi}{v_{\bar{a}}\left(k_{y}^{\prime}\right)} \bar{a}\left\langle\vec{k}^{\prime} \mid \vec{l}^{\prime}\right\rangle_{\bar{a}} V_{\bar{a}}^{\left(\vec{l}^{\prime}, j^{\prime}\right)}\left\langle j^{\prime}|\overline{\mathcal{G}}| j\right\rangle V_{a}^{(j, \vec{l})}{ }_{a}\langle\vec{l} \mid \vec{k}\rangle_{a}\right|^{2} .
\end{aligned}
$$

The transmission $\mathcal{T}^{(i)}$ is finally obtained by summing over all $k_{x}$ compatible with $\varepsilon_{a}(\vec{k})=\varepsilon$ and over all $k_{x}^{\prime}$ corresponding to "open" channels as

$$
\mathcal{T}_{a}^{(i)}(\varepsilon)=\sum_{\left\{k_{x}, k_{x}^{\prime}\right\}} \frac{v_{\bar{a}}\left(\varepsilon, k_{x}^{\prime}\right)}{v_{a}\left(\varepsilon, k_{x}\right)}\left|t\left(\varepsilon, k_{x}, k_{x}^{\prime}\right)\right|^{2},
$$

whereat the subscript $a$ can be dropped as $\mathcal{T}_{a}(\varepsilon)=\mathcal{T}_{\bar{a}}(\varepsilon)$ by unitarity of the $S$-matrix. In essence, this is a generalization of the calculation for end-coupled, one-dimensional leads presented in $\left[\mathrm{EMA}^{+} 05\right]$. The one-dimensional case can be obtained from (E.4) and E.5) by setting $N_{a}^{\text {con }}=1$, cf. chapter 4 .

\section{computing $\mathcal{T}^{(i i)}$ : vanishing corrections to the Landauer-Büttiker formula

In subsection 3.3.1, the calculation of the conductance within the approximate fRG scheme was explained and it was argued that there are no corrections to the transmission probability stemming from the vertex function $\gamma_{2}$, viz. $\mathcal{T}^{(i i)}=0$. This was first shown in the context of the fRG for a serial arrangement of quantum dots connected to the ends of semi-infinite, non-interacting hopping chains Ens05, p. 73 et seq.], [EMA $\left.{ }^{+} 05\right]$ and later extended to the coupling of arbitrary quantum dots [KEM06]. The basic scheme was introduced by Oguri Ogu01. In this appendix these calculations are generalized to a two-dimensional coupling setup, as presented in section chapter 4 and figure $4.1{ }^{1}$

Starting point is the Kubo formula for the conductance

$$
G=\lim _{\omega \rightarrow 0} \frac{C_{a \bar{a}}(\omega+\imath \eta)-C_{a \bar{a}}(0)}{\imath \omega}
$$

with $\eta \searrow 0$ and

$$
C_{a \bar{a}}\left(\imath \omega_{n}\right)=\int_{0}^{\beta} d \tau\left\langle\mathrm{T}_{\tau} J_{a}(\tau) J_{\bar{a}}(0)\right\rangle e^{\imath \omega_{n} \tau}
$$

\footnotetext{
${ }^{1}$ Any length scales appearing follow the numbering introduced in this figure.
} 


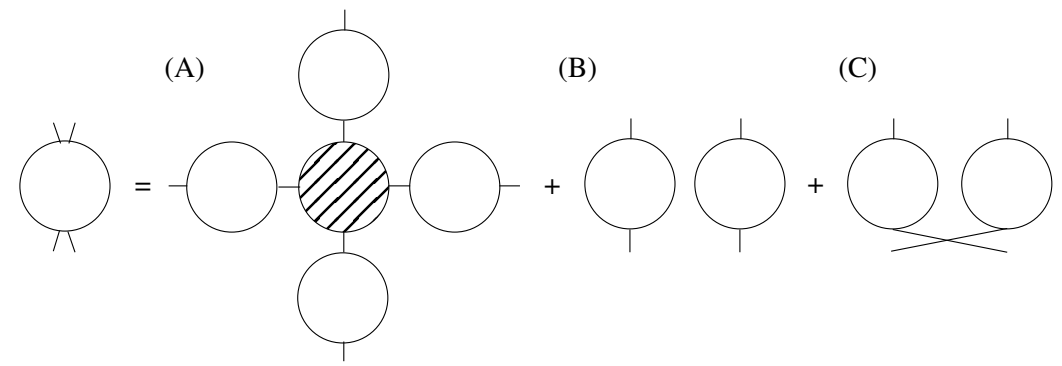

Figure E.1: Two-particle Green function $(\bigcirc$ with four legs) expressed in terms of one-particle Green function ( $\bigcirc$ with two legs) and vertex function $\gamma_{2}=$, cf. [NO98, eq. (2.158b)] in combination with [NO98, eq. (2.188a)].

cf. section 3.3.1. The current operators are explicitly given as

$$
J_{a}=\sum_{j=1}^{N_{a}^{\text {con }}} J_{a}^{(j)} \quad \text { with } \quad J_{a}^{(j)}=\imath t_{a}^{(j)}\left(c_{N_{a}^{\dagger}}^{\text {over }+j} d_{\left(j, N_{a}^{\text {lead }}\right)^{\mathrm{T}}, a}-\text { H.c. }\right)
$$

which upon inserting in (E.7) yields

$$
\begin{aligned}
C_{a \bar{a}}\left(\imath \omega_{n}\right)= & \sum_{j=1}^{N_{a}^{\text {con }}} \sum_{j^{\prime}=1}^{N_{\bar{a}}^{\text {con }}} \int_{0}^{\beta} d \tau\left\langle\mathrm{T}_{\tau} J_{a}^{(j)}(\tau) J_{\bar{a}}^{\left(j^{\prime}\right)}(0)\right\rangle e^{\imath \omega_{n} \tau} \\
= & \sum_{j=1}^{N_{a}^{\text {con }}} \sum_{j^{\prime}=1}^{N_{\bar{a}}^{\text {con }}} \int_{0}^{\beta} d \tau t_{a}^{(j)} t_{\bar{a}}^{\left(j^{\prime}\right)} \\
\times & \left(\mathcal{G}\left(\left\{N_{3}+j, \tau\right\},\left\{\left(j^{\prime}, N_{\bar{a}}^{\text {lead }}\right), 0\right\} ;\left\{\left(j, N_{a}^{\text {lead }}\right), \tau\right\},\left\{N_{1}+j^{\prime}, 0\right\}\right)\right. \\
& -\mathcal{G}\left(\left\{N_{3}+j, \tau\right\},\left\{N_{1}+j^{\prime}, 0\right\} ;\left\{\left(j, N_{a}^{\text {lead }}\right), \tau\right\},\left\{\left(j^{\prime}, N_{\bar{a}}^{\text {lead }}\right), 0\right\}\right) \\
& -\mathcal{G}\left(\left\{\left(j, N_{a}^{\text {lead }}\right), \tau\right\},\left\{\left(j^{\prime}, N_{\bar{a}}^{\text {lead }}\right), 0\right\} ;\left\{N_{3}+j, \tau\right\},\left\{N_{1}+j^{\prime}, 0\right\}\right) \\
& \left.+\mathcal{G}\left(\left\{\left(j, N_{a}^{\text {lead }}\right), \tau\right\},\left\{N_{1}+j^{\prime}, 0\right\} ;\left\{N_{3}+j, \tau\right\},\left\{\left(j^{\prime}, N_{\bar{a}}^{\text {lead }}\right), 0\right\}\right)\right)
\end{aligned}
$$

by definition of the two-particle Green functions.

Each two-particle Green function can be re-written as shown in fig. E.1 and $C_{a \bar{a}}\left(\imath \omega_{n}\right)$ can be split into a part $C_{a \bar{a}}^{(i)}\left(\imath \omega_{n}\right)$ only containing one-particle Green functions (diagrams B and C) and a part $C_{a \bar{a}}^{(i i)}\left(\imath \omega_{n}\right)$ containing all terms with the vertex 
function $\gamma_{2}$ (diagram A). Upon Fourier-transforming in each imaginary time variable and performing the time integration in each internal leg and over $\tau$, one obtains

$$
\begin{aligned}
C_{a \bar{a}}^{(i i)}\left(\imath \omega_{n}\right)= & \frac{1}{\beta^{2}} \sum_{\nu_{n}, \nu_{n}^{\prime}} \sum_{\{j\}=1}^{N} \sum_{j=1}^{N_{a}^{\text {con }}} \sum_{j^{\prime}=1}^{N_{\bar{a}}^{\text {con }}} t_{a}^{(j)} t_{\bar{a}}^{\left(j^{\prime}\right)} \\
& {\left[\mathcal{G}\left(\left(j^{\prime}, N_{\bar{a}}^{\text {lead }}\right), j_{2} ; \imath \nu_{n}\right) \mathcal{G}\left(j_{4}, N_{1}+j^{\prime} ; \imath \omega_{n}+\imath \nu_{n}\right)\right.} \\
& \left.\quad-\mathcal{G}\left(N_{1}+j^{\prime}, j_{2} ; \imath \omega_{n}\right) \mathcal{G}\left(j_{4},\left(j^{\prime}, N_{\bar{a}}^{\text {lead }}\right) ; \imath \omega_{n}+\imath \nu_{n}\right)\right] \\
& \times \gamma_{2}\left(\left\{j_{1}, \imath \omega_{n}+\imath \nu_{n}\right\},\left\{j_{2}, \imath \nu_{n}^{\prime}\right\} ;\left\{j_{3}, \imath \omega_{n}\right\},\left\{j_{4}, \imath \omega_{n}+\imath \nu_{n}^{\prime}\right\}\right) \\
& \times\left[\mathcal{G}\left(N_{3}+j, j_{1} ; \imath \omega_{n}+\imath \nu_{n}^{\prime}\right) \mathcal{G}\left(j_{3},\left(j, N_{a}^{\text {lead }}\right) ; \imath \nu_{n}^{\prime}\right)\right. \\
& \left.\quad-\mathcal{G}\left(\left(j, N_{a}^{\text {lead }}\right), j_{1} ; \imath \omega_{n}+\imath \nu_{n}^{\prime}\right) \mathcal{G}\left(j_{3}, N_{3}+j ; \imath \nu_{n}^{\prime}\right)\right]
\end{aligned}
$$

with $j=\left\{j_{1}, j_{2}, j_{3}, j_{4}\right\}$. The Green functions involving lead operators can be projected onto the wire using the projections $(\mathrm{B} .2)$ and $(\mathrm{B} .3)$, e. g.

$$
\mathcal{G}\left(\left(j^{\prime}, N_{a}^{\text {lead }}\right), j_{2} ; \imath \omega_{n}\right)=-\sum_{\tilde{\jmath}=1}^{N_{a}^{\text {lead }}} t_{a}^{(\tilde{\jmath})} \mathcal{G}^{2 \mathrm{~d}}\left(\left(j^{\prime}, N_{a}^{\text {lead }}\right),\left(\tilde{\jmath}, N_{a}^{\text {lead }}\right) ; \imath \omega_{n}\right) \mathcal{G}\left(N_{1}+\tilde{\jmath}, j_{2} ; \imath \omega_{n}\right)
$$

which upon insertion into $(\overline{E .8})$ and after some rearrangement gives

$$
\begin{aligned}
C_{a \bar{a}}^{(i i)}\left(\imath \omega_{n}\right)=-\frac{1}{\beta} \sum_{\nu_{n}} \sum_{j_{2}, j_{4}=1}^{N} \sum_{j^{\prime}, \tilde{\jmath}=1}^{N_{\bar{a}}^{\text {lead }}} t_{\bar{a}}^{\left(j^{\prime}\right)} t_{\bar{a}}^{(\tilde{\jmath})} \mathcal{G}\left(j_{4}, N_{1}+j^{\prime} ; \imath \omega_{n}+\imath \nu_{n}\right) \lambda_{\bar{a}}^{\left(j^{\prime}, \tilde{\jmath}\right)}\left(\imath \nu_{n}, \imath \omega_{n}+\imath \nu_{n}\right) \\
\times \mathcal{G}\left(N_{1}+\tilde{\jmath}, j_{2} ; \imath \nu_{n}\right) \Lambda_{a}^{\left(j_{2}, j_{4}\right)}\left(\imath \omega_{n} ; \imath \nu_{n}\right)
\end{aligned}
$$

with the definitions

$$
\begin{aligned}
\lambda_{\bar{a}}^{\left(j^{\prime}, \tilde{\jmath}\right)}\left(\imath \nu_{n}, \imath \omega_{n}+\imath \nu_{n}\right):= & \mathcal{G}^{2 \mathrm{~d}}\left(\left(j^{\prime}, N_{\bar{a}}^{\text {lead }}\right),\left(\tilde{\jmath}, N_{\bar{a}}^{\text {lead }}\right) ; \imath \nu_{n}\right)- \\
& \mathcal{G}^{2 \mathrm{~d}}\left(\left(j^{\prime}, N_{\bar{a}}^{\text {lead }}\right),\left(\tilde{\jmath}, N_{\bar{a}}^{\text {lead }}\right) ; \imath \nu_{n}+\imath \omega_{n}\right)
\end{aligned}
$$

and

$$
\begin{aligned}
\Lambda_{a}^{\left(j_{2}, j_{4}\right)}\left(\imath \omega_{n}, \imath \nu_{n}\right):= & -\frac{1}{\beta} \sum_{\imath \nu_{n}^{\prime}} \sum_{j_{1}, j_{3}=1}^{N} \sum_{j, \tilde{\tilde{j}}=1}^{N_{a}^{\text {lead }}} t_{a}^{\left(j^{\prime}\right)} t_{a}^{(\tilde{\tilde{\jmath}})} \\
& \times \gamma_{2}\left(\left\{j_{1}, \imath \omega_{n}+\imath \nu_{n}\right\},\left\{j_{2}, \imath \nu_{n}^{\prime}\right\} ;\left\{j_{3}, \imath \omega_{n}\right\},\left\{j_{4}, \imath \omega_{n}+\imath \nu_{n}^{\prime}\right\}\right) \\
& \times \mathcal{G}\left(j_{3}, N_{3}+j ; \imath \nu_{n}^{\prime}\right) \lambda_{R}^{(j, \tilde{\tilde{\jmath}})}\left(\imath \nu_{n}^{\prime}, \imath \omega_{n}+\imath \nu_{n}^{\prime}\right) \mathcal{G}\left(N_{3}+\tilde{\tilde{\jmath}}, j_{1} ; \imath \nu_{n}^{\prime}+\imath \omega\right) .
\end{aligned}
$$


The complicated frequency structure of $\Lambda$ can significantly be reduced by using the fact, that $\gamma_{2}$ is frequency independent in the approximate fRG scheme, cf. section 3.1.2. The sum over $\nu_{n}^{\prime}$ can then be cast into an integration on the real axis Mah00, p. 511], giving

$$
\begin{aligned}
\Lambda_{a}^{\left(j_{2}, j_{4}\right)}\left(\imath \omega_{n}\right)= & -\sum_{j_{1}, j_{3}=1}^{N} \gamma_{2}\left(j_{1}, j_{2} ; j_{3}, j_{4}\right) \sum_{j, \tilde{\tilde{\jmath}}=1}^{N_{a}^{\text {lead }}} t_{a}^{\left(j^{\prime}\right)} t_{a}^{(\tilde{\tilde{\jmath}})} \frac{1}{2 \pi \imath} \int_{-\infty}^{\infty} d \varepsilon f(\varepsilon) \\
& {\left[\mathcal{G}\left(j_{3}, N_{3}+j ; \varepsilon+\imath \eta\right) \lambda_{a}^{(j, \tilde{\tilde{\jmath}})}\left(\varepsilon+\imath \eta, \varepsilon+\imath \omega_{n}\right) \mathcal{G}\left(N_{3}+\tilde{\tilde{\jmath}}, j_{1} ; \varepsilon+\imath \omega_{n}\right)\right.} \\
& -\mathcal{G}\left(j_{3}, N_{3}+j ; \varepsilon-\imath \eta\right) \lambda_{a}^{(j, \tilde{\tilde{\jmath}})}\left(\varepsilon-\imath \eta, \varepsilon+\imath \omega_{n}\right) \mathcal{G}\left(N_{3}+\tilde{\tilde{\jmath}}, j_{1} ; \varepsilon+\imath \omega_{n}\right) \\
& +\mathcal{G}\left(j_{3}, N_{3}+j ; \varepsilon-\imath \omega_{n}\right) \lambda_{a}^{(j, \tilde{\tilde{\jmath}})}\left(\varepsilon-\imath \omega_{n}, \varepsilon+\imath \eta\right) \mathcal{G}\left(N_{3}+\tilde{\tilde{\jmath}}, j_{1} ; \varepsilon+\imath \eta\right) \\
& \left.-\mathcal{G}\left(j_{3}, N_{3}+j ; \varepsilon-\imath \omega_{n}\right) \lambda_{a}^{(j, \tilde{\tilde{\jmath}})}\left(\varepsilon-\imath \omega_{n}, \varepsilon-\imath \eta\right) \mathcal{G}\left(N_{3}+\tilde{\tilde{\jmath}}, j_{1} ; \varepsilon-\imath \eta\right)\right]
\end{aligned}
$$

with $\eta \searrow 0$. Since eventually the limit $C_{a \bar{a}}^{(i i)}(\omega \rightarrow 0)$ contributes to $G$, cf. (E.6), the leading $\omega_{n}$-behavior of the various terms is determined. First

$$
\begin{aligned}
\lambda_{a}^{(j \tilde{\tilde{\jmath}})}\left(\varepsilon \pm \imath \omega_{n}, \varepsilon \pm \imath \eta\right) & =\sum_{k_{x}}\left\langle j \mid k_{x}\right\rangle\left\langle k_{x} \mid \tilde{\tilde{\jmath}}\right\rangle\left(\left\langle N_{a}^{\text {lead }}\left|\mathcal{G}^{1 \mathrm{~d}}\left(\tilde{\varepsilon} \pm \imath \omega_{n}\right)\right| N_{a}^{\text {lead }}\right\rangle\right. \\
& \rightarrow \mathcal{O}\left(\omega_{n}\right), \\
& \left.\left.\rightarrow N_{a}^{\text {lead }}\left|\mathcal{G}^{1 \mathrm{~d}}(\tilde{\varepsilon} \pm \imath \eta)\right| N_{a}^{\text {lead }}\right\rangle\right) \\
\lambda_{a}^{(j, \tilde{\tilde{\jmath}})}\left(\varepsilon-\imath \omega_{n}, \varepsilon+\imath \eta\right) & =\lambda_{a}^{(j, \tilde{\tilde{\jmath}})}\left(\varepsilon-\imath \eta, \varepsilon+\imath \omega_{n}\right) \\
& \rightarrow \mathcal{O}\left(\omega_{n}^{0}\right)
\end{aligned}
$$

as follows from the definition (E.10) in combination with (D.6). The definition of $\tilde{\varepsilon}$ follows from the definition of $\tilde{z}$ in section D.4. The limiting behavior of $\mathcal{G}^{1 \mathrm{~d}}$ follows from expanding the retarded and advanced (D.2) in $\pm \imath \eta$ respectively, cf. [Ens05, p. 76]. This shows, that the first and last term in (E.13) are by an order $\mathcal{O}\left(\omega_{n}\right)$ smaller than the second and third. Discarding them, performing the continuation $\imath \omega_{n} \rightarrow \omega+\imath \eta$ and shifting $\varepsilon$ by $\omega$ in the resulting second term gives

$$
\begin{aligned}
\Lambda_{a}^{\left(j_{2}, j_{4}\right)}(\omega+\imath \eta) \approx & \sum_{j_{1}, j_{3}=1}^{N} \gamma_{2}\left(j_{1}, j_{2} ; j_{3}, j_{4}\right) \sum_{j, \tilde{\tilde{\jmath}}=1}^{N_{a}^{\text {lead }}} t_{a}^{\left(j^{\prime}\right)} t_{a}^{(\tilde{\tilde{\jmath}})} \frac{\imath}{2 \pi} \int_{-\infty}^{\infty} d \varepsilon \\
& {[f(\varepsilon+\omega)-f(\varepsilon)] \mathcal{G}\left(j_{3}, N_{3}+j ; \omega-\imath \eta\right) \lambda_{a}^{(j, \tilde{\tilde{\jmath}})}(\varepsilon-\imath \eta, \varepsilon+\omega+\imath \eta) } \\
& \quad \times \mathcal{G}\left(N_{3}+\tilde{\tilde{\jmath}}, j_{1} ; \varepsilon+\omega+\imath \eta\right) \\
\rightarrow & \mathcal{O}(\omega) .
\end{aligned}
$$

Going back to $(\mathrm{E} .9)$ in the light of this result shows, that $C_{a \bar{a}}^{(i i)}(\omega+\imath \eta)=\mathcal{O}\left(\omega^{2}\right)$ and thus

$$
\lim _{\omega \rightarrow 0} \frac{C_{a \bar{a}}^{(i i)}(\omega+\imath \eta)-C_{a \bar{a}}^{(i i)}(0)}{\imath \omega} \rightarrow \frac{\mathcal{O}\left(\omega^{2}\right)}{\omega} \rightarrow 0 .
$$


This shows that within the approximation of a frequency independent $\gamma_{2}$, the contribution of $\gamma_{2}$ to the conductance vanishes or in other words $\mathcal{T}^{(i i)}=0$. 


\section{details of the perturbation theory for the single dot $\mathrm{F}$}

This appendix contains details, which were omitted in the calculations of chapter 5. All results have been checked by numerical investigation of the basic formula.

\section{perturbative calculation of $\Sigma^{\operatorname{dot}}(z) \quad \mathrm{F} .1$}

By definition of the self-energy $\Sigma^{\operatorname{dot}}(z)$ (e. g. [NO98, p. 113]), the full Green function $\mathcal{G}^{\text {dot }}$ on the dot is given by

$$
\mathcal{G}^{\operatorname{dot}}(z)=\left(\left[\mathcal{G}_{0}^{\operatorname{dot}}\right]^{-1}(z)-\Sigma^{\operatorname{dot}}(z)\right)^{-1}
$$

with $\mathcal{G}_{0}^{\operatorname{dot}}(z)=1 /\left(z-V_{g}\right)$ being the Green function of the isolated $\operatorname{dot}\left(t_{L}=t_{R}=0\right)$. This reads in terms of the Dyson equation [NO98, p. 113]

$$
\mathcal{G}^{\text {dot }}=\mathcal{G}_{0}^{\text {dot }}+\mathcal{G}_{0}^{\text {dot }} \Sigma^{\text {dot }} \mathcal{G}_{0}^{\text {dot }}+\mathcal{G}_{0}^{\text {dot }} \Sigma^{\text {dot }} \mathcal{G}_{0}^{\text {dot }} \Sigma^{\text {dot }} \mathcal{G}_{0}^{\text {dot }}+\ldots
$$

Complementary, the equation of motion [BF04, p. 140 et. seq.] for $\mathcal{G}^{\text {dot }}(z)$ gives the closed set

$$
\begin{aligned}
\left(z-V_{g}\right) \mathcal{G}^{\mathrm{dot}}(z)-\sum_{a=L, R} t_{a} \mathcal{G}_{a}^{\mathrm{dot}-\mathrm{end}}(z) & =1 \\
\left(z-V_{g}\right) \mathcal{G}_{a}^{\text {dot-end }}(z)-t_{a} \mathcal{G}_{a}^{\text {end }}(z) & =0 .
\end{aligned}
$$

with the solution

$$
\mathcal{G}^{\operatorname{dot}}(z)=\mathcal{G}_{0}^{\text {dot }}+\mathcal{G}_{0}^{\text {dot }}\left[\sum_{a=L, R} t_{a}^{2} \mathcal{G}_{a}^{\text {end }}(z)\right] \mathcal{G}_{0}^{\text {dot }}
$$

Comparing (F.1) and (F.2) yields the self-energy to leading order in $\Gamma:=\sum_{a=L, R} t_{a}^{2}$,

$$
\Sigma^{\operatorname{dot}}(z)=\sum_{a=L, R} t_{a}^{2} \mathcal{G}_{a}^{\mathrm{end}}(z)=\Gamma \mathcal{G}^{\mathrm{end}}(z)
$$

whereat $\mathcal{G}_{a}^{\text {end }}(z)=\mathcal{G}_{\bar{a}}^{\text {end }}(z)$ was used. 


\section{charging and width}

The charging of the dot is given by, cf. eq. (5.5)

$$
n^{\mathrm{dot}}\left(V_{g}\right)=\int_{-\omega_{c}}^{0} d \omega \frac{\Gamma \rho^{\mathrm{end}}(\omega)}{\left(\omega-V_{g}-\Gamma \mathfrak{R e} \mathcal{G}_{\mathrm{ret}}^{\text {end }}(\omega)\right)^{2}+\left(\Gamma \rho^{\mathrm{end}}(\omega)\right)^{2}} .
$$

To ease the notation, $\alpha_{\text {end }}=1 / K-1$, cf. section 2.1, is used in the following.

the regime $K \in(0,1 / 2)\left[\alpha_{\text {end }}>1\right]$

The charging in leading order in $\Gamma$ is given by

$$
n^{\operatorname{dot}}\left(V_{g}\right)=\frac{\Gamma}{2 K \omega_{c}^{\alpha_{\text {end }}+1}} \int_{0}^{\omega_{c}} d \omega \frac{\omega^{\alpha_{\text {end }}}}{\left(\omega+V_{g}\right)^{2}},
$$

cf. eqs. (5.7) and (5.3). The integral can be done, yielding

$$
\begin{aligned}
n^{\operatorname{dot}}\left(V_{g}\right)= & \frac{\Gamma}{2 K \omega_{c}^{\alpha_{\text {end }}+1} \frac{1}{V_{g}}} \\
& \times\left[\frac{\omega_{c}^{\alpha_{\text {end }}}}{1+V_{g} / \omega_{c}}+\omega_{c}^{\alpha_{\text {end }}} \alpha_{\text {end }} \Phi\left(-V_{g} / \omega_{c}, 1,-\alpha_{\text {end }}\right)+\frac{\pi \alpha_{\text {end }}}{\sin \left(\alpha_{\text {end }} \pi\right)}\left(V_{g}\right)^{\alpha_{\text {end }}}\right] \\
= & \frac{\Gamma}{2 K}\left[\omega_{c}^{-2} \sum_{n=1}^{\infty}(-1)^{n}\left(\frac{V_{g}}{\omega_{c}}\right)^{n-1} \frac{n}{n-\alpha_{\text {end }}}+\frac{\pi \alpha_{\text {end }}}{\omega_{c}^{2} \sin \left(\alpha_{\text {end }} \pi\right)}\left(\frac{V_{g}}{\omega_{c}}\right)^{\alpha_{\text {end }}-1}\right]
\end{aligned}
$$

with the function $\Phi(z, s, v)=\sum_{n=0}^{\infty}(v+n)^{-s} z^{n}$ [EMOT53, p. 27]. The second equality was derived by inserting $\Phi$ and combining the first two terms in the first line. In the limit $V_{g} \searrow 0$, only the $n=1$-term in the sum survives $\left(\alpha_{\text {end }}>1\right)$ and thus

$$
\begin{aligned}
n^{\operatorname{dot}}\left(V_{g} \searrow 0\right) & =\frac{\Gamma}{2 K} \frac{\omega_{c}^{-2}}{\alpha_{\text {end }}-1} \\
& =\frac{\Gamma}{2 \omega_{c}^{2}} \frac{\alpha_{\text {end }}+1}{\alpha_{\text {end }}-1}=\frac{\Gamma}{\omega_{c}^{2}(2-4 K)} .
\end{aligned}
$$

The jump $\Delta$ follows as $\Delta=1-2 n^{\operatorname{dot}}\left(V_{g} \searrow 0\right)=1-\frac{\Gamma}{\omega_{c}^{2}(1-2 K)}$. The leading corrections to $n^{\operatorname{dot}}\left(V_{g} \searrow 0\right)$ for finite $V_{g} \ll 1$ can be extracted from (F.4). For $1 / 3<K<1 / 2$, i. e. $1<\alpha_{\text {end }}<2$, the second term in $\left(\right.$ F.4 dominates for $V_{g} \ll 1$ and thus

$$
n^{\operatorname{dot}}\left(V_{g} \searrow 0\right)-n^{\operatorname{dot}}\left(V_{g}\right) \sim\left(\frac{V_{g}}{\omega_{c}}\right)^{\alpha_{\text {end }}-1}=\left(\frac{V_{g}}{\omega_{c}}\right)^{1 / K-2} .
$$

For $K<1 / 3\left[\alpha_{\text {end }}>2\right]$, the (linear) $n=2$-term in the sum in $(\mathrm{F} .4)$ dominates for $V_{g} \ll 1$, giving

$$
n^{\operatorname{dot}}\left(V_{g} \searrow 0\right)-n^{\operatorname{dot}}\left(V_{g}\right) \sim\left(\frac{V_{g}}{\omega_{c}}\right) .
$$


At the point $K=1 / 3\left[\alpha_{\text {end }}=2\right]$, eq. (F.4), is split up as

$$
\begin{aligned}
n^{\operatorname{dot}}\left(V_{g}\right)= & n^{\operatorname{dot}}\left(V_{g} \searrow 0\right) \\
& +\frac{\Gamma}{2 K} \lim _{\alpha_{\text {end }} \rightarrow 2}\left[\omega_{c}^{-2}\left(\frac{V_{g}}{\omega_{c}}\right) \frac{2}{2-\alpha_{\text {end }}}+\frac{\pi \alpha_{\text {end }}}{\omega_{c}^{2} \sin \left(\alpha_{\text {end }} \pi\right)}\left(\frac{V_{g}}{\omega_{c}}\right)^{\alpha_{\text {end }}-1}\right] \\
& +\mathcal{O}\left(V_{g}^{2}\right) .
\end{aligned}
$$

Expanding $1 / \sin \left(\alpha_{\text {end }} \pi\right) \approx 1 /\left(\pi \alpha_{\text {end }}\right)$ and rearranging the terms in the bracket gives

$n^{\operatorname{dot}}\left(V_{g}\right)=n^{\operatorname{dot}}\left(V_{g} \searrow 0\right)-\frac{\Gamma}{2 K} \lim _{\alpha_{\text {end }} \rightarrow 2}\left(\left(\frac{V_{g}}{\omega_{c}}\right) \frac{\alpha_{\text {end }}}{2-\alpha_{\text {end }}}\left[1-\left(\frac{V_{g}}{\omega_{c}}\right)^{\alpha_{\text {end }}-2}\right]\right)+\mathcal{O}\left(V_{g}^{2}\right)$

With $\lim _{a \rightarrow 0}\left(1-x^{a}\right) / a=\ln x$ finally

$$
n^{\operatorname{dot}}\left(V_{g} \searrow 0\right)-n^{\operatorname{dot}}\left(V_{g}\right) \sim\left(\frac{V_{g}}{\omega_{c}}\right) \ln \left(\frac{V_{g}}{\omega_{c}}\right)
$$

is obtained. All results for $K \in(0,1 / 2)\left[\alpha_{\text {end }}>1\right]$ are summarized in (5.8).

the regime $K \in(1 / 2,1]\left[\alpha_{\text {end }}<1\right]$

Inserting the appropriate real part (5.4) and the imaginary part (5.3) of $\mathcal{G}_{\text {ret }}^{\text {end }}$ in F.3 and taking the derivative with respect to $V_{g}$ gives

$$
\frac{d n^{\mathrm{dot}}}{d V_{g}}=\int_{0}^{\omega_{c}} d \omega \frac{2 \Gamma c_{1} \omega^{\alpha_{\mathrm{end}}}\left(\omega+V_{g}+\Gamma c_{1} \omega^{\alpha_{\mathrm{end}}}\right)}{\left[\left(\omega+V_{g}+\Gamma c_{1} \omega^{\alpha_{\mathrm{end}}}\right)+\Gamma^{2} c_{1}^{2} \omega^{2 \alpha_{\mathrm{end}}}\right]^{2}}
$$

with $c_{1}=\frac{1}{2 K \omega^{\alpha} \text { end }^{+1}}$ to condense the notation. The lower integration limit dominates the integral, therefore the terms linear in $\omega$ are neglected $\left(\alpha_{\text {end }}<1\right)$. Substituting $\omega^{\alpha_{\text {end }}}=V_{g} \Omega$ gives

$$
\frac{d n^{\mathrm{dot}}}{d V_{g}} \sim V_{g}^{1 / \alpha_{\mathrm{end}}-2} \int_{0}^{\frac{\omega_{c}^{\alpha_{\mathrm{end}}}}{V_{g}}} d \Omega \frac{c_{1} \Omega^{1 / \alpha_{\mathrm{end}}}\left(\Gamma c_{1} \Omega+1\right)}{\left[\left(\Gamma c_{1} \Omega+1\right)^{2}+\Gamma^{2} c_{1}^{2} \Omega^{2}\right]^{2}}
$$

with the upper integration limit dominating the integral, being $\sim \Omega^{1 / \alpha_{\text {end }}-3}$. For small $V_{g}$ follows $\frac{d n^{\mathrm{dot}}}{d V_{g}} \sim$ const. $+c_{2} V_{g}^{1 / \alpha_{\text {end }}-2}$ with some $c_{2} \in \mathbb{R}$. Thus, for $\alpha_{\text {end }}<$ $1 / 2$, the leading term of $n^{\operatorname{dot}}\left(V_{g}\right)$ is linear, while for $\alpha_{\text {end }}>1 / 2$, the anomalous term $V_{g}^{1 / \alpha_{\text {end }}-1}=V_{g}^{(2 K-1) /(1-K)}$ determines the leading behavior. The logarithmic corrections at $\alpha_{\text {end }}=1 / 2$ were extracted numerically.

The width $W$ was defined by $W=2 V_{g}^{\text {ch }}$ with $n^{\operatorname{dot}}\left(V_{g}^{\text {ch }}\right)=1 / 4$, cf. section 5.2 For $\Gamma \ll 1$, the terms in the denominator containing $\Gamma$ are neglected, yielding

$$
n^{\operatorname{dot}}\left(V_{g}^{\mathrm{ch}}\right) \sim \Gamma \int_{0}^{\omega_{c}} d \omega \frac{\omega^{\alpha_{\text {end }}}}{\left(\omega+V_{g}^{\text {ch }}\right)^{2}}
$$


The integral is known from above and solving for $\Gamma$ gives

$$
\frac{1}{\Gamma} \sim\left[\omega_{c}^{-2} \sum_{n=1}^{\infty}(-1)^{n}\left(\frac{V_{g}^{\mathrm{ch}}}{\omega_{c}}\right)^{n-1} \frac{n}{n-\alpha_{\mathrm{end}}}+\frac{\pi \alpha_{\mathrm{end}}}{\omega_{c}^{2} \sin \left(\alpha_{\mathrm{end}} \pi\right)}\left(\frac{V_{g}^{\mathrm{ch}}}{\omega_{c}}\right)^{\alpha_{\mathrm{end}}-1}\right],
$$

which yields $\frac{1}{\Gamma} \sim \frac{\pi \alpha_{\text {end }}}{\omega_{c}^{2} \sin \left(\alpha_{\text {end }} \pi\right)}\left(V_{g}^{\text {ch }}\right)^{\alpha_{\text {end }}-1}+\mathcal{O}\left(V_{g}^{\text {ch }}\right)$ and thus

$$
W=2 V_{g}^{\mathrm{ch}} \sim \Gamma^{1 /\left(1-\alpha_{\mathrm{end}}\right)}=\Gamma^{K /(2 K-1)},
$$

cf. eq. (5.9). 


\section{parallel double quantum dot with non-interacting leads}

In this appendix, the conductance and local spectral function of the parallel double quantum dot with non-interacting leads, that is the Hamiltonian (5.1) with $h^{\text {dot }}$ given by (5.10) and $U=0$ is briefly reviewed. Detailed studies with a broader perspective are [KEM06, $\mathrm{KHW}^{+} 07$, KSAEW07].

Calculating the retarded Green function $\mathcal{G}_{\text {ret }}\left(N_{L}, N_{R}, \omega\right)$ using an equation of motion [BF04, p. 140 et. seq.], the conductance $G\left(V_{g}\right)$ follows (cf. [EMA ${ }^{+}$05]) as

$$
\begin{aligned}
& G\left(V_{g}\right) / G_{0}=4\left|\mathcal{G}_{\text {ret }}\left(N_{L}, N_{R}, \omega=0\right)\right|^{2}= \\
& \frac{4\left(t_{L}^{(1)} t_{R}^{(1)}\left(V_{g}+\delta\right)+t_{L}^{(2)} t_{R}^{(2)} V_{g}\right)^{2}}{\left(\left[t_{L}^{(1)} t_{R}^{(2)}-t_{L}^{(2)} t_{R}^{(1)}\right]^{2}-V_{g}\left(V_{g}+\delta\right)\right)^{2}+\left(V_{g}\left(\left[t_{L}^{(2)}\right]^{2}+\left[t_{R}^{(2)}\right]^{2}\right)+\left(V_{g}+\delta\right)\left(\left[t_{L}^{(1)}\right]^{2}+\left[t_{R}^{(1)}\right]^{2}\right)\right)^{2}} .
\end{aligned}
$$

For tuned dots, i. e. $\delta=0$, the conductance shows three basic peak forms around $V_{g}=0$ differing by their behavior at $G\left(V_{g}=0\right)$. Exemplary curves are shown in fig. G.1.

In the non-generic case of couplings obeying $t_{L}^{(1)} t_{R}^{(2)}-t_{L}^{(2)} t_{R}^{(1)}=0$, the conductance simplifies to

$$
G\left(V_{g}\right) / G_{0}=\frac{4\left(t_{L}^{(1)} t_{R}^{(1)}+t_{L}^{(2)} t_{R}^{(2)}\right)^{2}}{V_{g}^{2}+\left(\left[t_{L}^{(2)}\right]^{2}+\left[t_{R}^{(2)}\right]^{2}+\left[t_{L}^{(1)}\right]^{2}+\left[t_{R}^{(1)}\right]^{2}\right)^{2}}
$$

thus, with non-vanishing $G\left(V_{g}=0\right) / G_{0}=\frac{4\left(t_{L}^{(1)} t_{R}^{(1)}+t_{L}^{(2)} t_{R}^{(2)}\right)^{2}}{\left(\left[t_{L}^{(2)}\right]^{2}+\left[t_{R}^{(2)}\right]^{2}+\left[t_{L}^{(1)}\right]^{2}+\left[t_{R}^{(1)}\right]^{2}\right)^{2}}<1$ [black line in fig. G.1. For symmetric coupling of each dot, i. e. $t_{L}^{(1)}=t_{R}^{(1)}$ and $t_{L}^{(2)}=t_{R}^{(2)}$ (which automatically implies $t_{L}^{(1)} t_{R}^{(2)}-t_{L}^{(2)} t_{R}^{(1)}=0$ ), the conductance at vanishing gate voltage shows a perfect resonance, $G\left(V_{g}=0\right) / G_{0}=1$ [blue line in fig. G.1]. The third basic form is defined by couplings with $t_{L}^{(1)} t_{R}^{(2)}-t_{L}^{(2)} t_{R}^{(1)} \neq 0$, which leads to a sharp anti-resonance around $V_{g}=0$ with $G\left(V_{g}=0\right) / G_{0}=0$ [red line in fig. G.1]. 


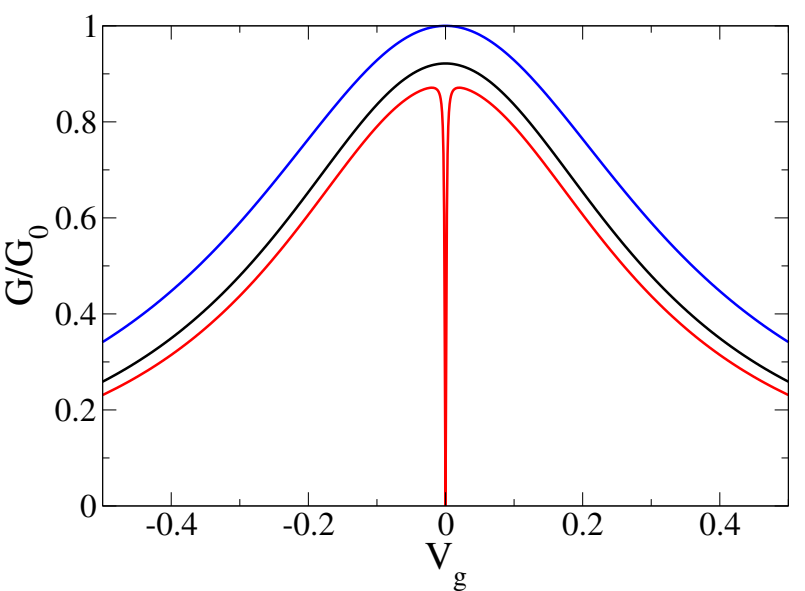

Figure G.1: Conductance $G$ as function of gate voltage $V_{g}$ through tuned $(\delta=0)$ parallel double quantum dots coupled to non-interacting leads with $t_{L}^{(1)}=t_{R}^{(1)}=t_{L}^{(2)}=t_{R}^{(2)}=0.3$ [blue], $t_{L}^{(1)}=0.4, t_{R}^{(1)}=0.3, t_{L}^{(2)}=0.2, t_{R}^{(2)}=$ 0.15 (i. e. $\left.t_{L}^{(1)} t_{R}^{(2)}-t_{L}^{(2)} t_{R}^{(1)}=0\right)$ [black] and $t_{L}^{(1)}=0.4, t_{R}^{(1)}=0.3, t_{L}^{(2)}=0.2$, $t_{R}^{(2)}=0.1$ (i. e. $t_{L}^{(1)} t_{R}^{(2)}-t_{L}^{(2)} t_{R}^{(1)} \neq 0$ ) [red].

For detuned dots, i. e. $\delta \neq 0$, the $G\left(V_{g}\right)$-curve shows two resonance peaks at vanishing eigenenergies of the isolated $\operatorname{dots}\left(V_{g}=0\right.$ and $\left.V_{g}=-\delta\right)$. Their height is given by

$$
\begin{gathered}
G\left(V_{g}=0\right) / G_{0}=\frac{4 \delta^{2}\left(t_{L}^{(1)} t_{R}^{(1)}\right)^{2}}{\left[t_{L}^{(1)} t_{R}^{(2)}-t_{L}^{(2)} t_{R}^{(1)}\right]^{4}+\delta^{2}\left(\left[t_{L}^{(1)}\right]^{2}+\left[t_{R}^{(1)}\right]^{2}\right)^{2}} \text { and } \\
G\left(V_{g}=-\delta\right) / G_{0}=\frac{4 \delta^{2}\left(t_{L}^{(2)} t_{R}^{(2)}\right)^{2}}{\left[t_{L}^{(1)} t_{R}^{(2)}-t_{L}^{(2)} t_{R}^{(1)}\right]^{4}+\delta^{2}\left(\left[t_{L}^{(2)}\right]^{2}+\left[t_{R}^{(2)}\right]^{2}\right)^{2}}
\end{gathered}
$$

respectively. Exemplary curves are shown in fig. G.2. Eqs. (G.2a) and (G.2b show that, if each dot is coupled symmetrically $\left(t_{L}^{(1)}=t_{R}^{(1)}\right.$ and $\left.t_{L}^{(2)}=t_{R}^{(2)}\right)$, the peaks show perfect conductance, $G\left(V_{g}\right) / G_{0}=1$ and $G\left(V_{g}=-\delta\right) / G_{0}=1$ [green line in fig. G.2. For any other relation between the couplings, the conductance is nonperfect, $G\left(V_{g}\right) / G_{0}<1$ and $G\left(V_{g}=-\delta\right) / G_{0}<1$ [red and orange line in fig. G.2. This even holds, if one of the two dots is coupled symmetrically and the other asymmetrically (implying $t_{L}^{(1)} t_{R}^{(2)}-t_{L}^{(2)} t_{R}^{(1)} \neq 0$ ) [orange line in fig. G.2, the peak at $V_{g}=0$ is 0.99999375 , cf. (G.2a)]. Between the two resonance peaks, the conductance vanishes at $V_{g}=\frac{-\delta t_{L}^{(1)} t_{R}^{(1)}}{t_{L}^{(2)} t_{R}^{(2)}+t_{L}^{(1)} t_{R}^{(1)}}$.

The local spectral functions $\rho(\omega)$ on the dots and on the sites next to the dots follow from equation (3.20) by taking the imaginary part of the respective Green function, which can directly be calculated from their equation of motion.

Fig. G.3 illustrates, that in the case of tuned dots, the line shapes of the local spectral functions differ significantly for $t_{L}^{(1)} t_{R}^{(2)}-t_{L}^{(2)} t_{R}^{(1)}=0$ [dashed lines] and $t_{L}^{(1)} t_{R}^{(2)}-t_{L}^{(2)} t_{R}^{(1)} \neq 0$ [solid lines]. The local spectral functions on the dots [black: dot 1 , red: $\operatorname{dot} 2] t_{L}^{(1)} t_{R}^{(2)}-t_{L}^{(2)} t_{R}^{(1)}=0$ have a Lorentzian shape centered close to $\omega=V_{g}$ with width determined by the dot-lead coupling strengths. Apart from this continuous spectrum, two bound states exist at $\omega=V_{g}$ with weight $\frac{\left[t_{L}^{(2 / 1)}\right]^{2}+\left[t_{R}^{(2 / 1)}\right]^{2}}{\left[t_{L}^{(1)}\right]^{2}+\left[t_{R}^{(1)}\right]^{2}+\left[t_{L}^{(2)}\right]^{2}+\left[t_{R}^{(2)}\right]^{2}}(\operatorname{dot}$ 
Figure G.2: Conductance $G$ as function of gate voltage $V_{g}$ through detuned $(\delta=0.5)$ parallel double quantum dots coupled to non-interacting leads with $t_{L}^{(1)}=t_{R}^{(1)}=0.1, t_{L}^{(2)}=$ $t_{R}^{(2)}=0.2$ [green], $t_{L}^{(1)}=0.4, t_{R}^{(1)}=0.3$, $t_{L}^{(2)}=0.2, t_{R}^{(2)}=0.1$ [red] and $t_{L}^{(1)}=$ $t_{R}^{(1)}=0.1, t_{L}^{(2)}=0.1, t_{R}^{(2)}=0.15$ [orange].
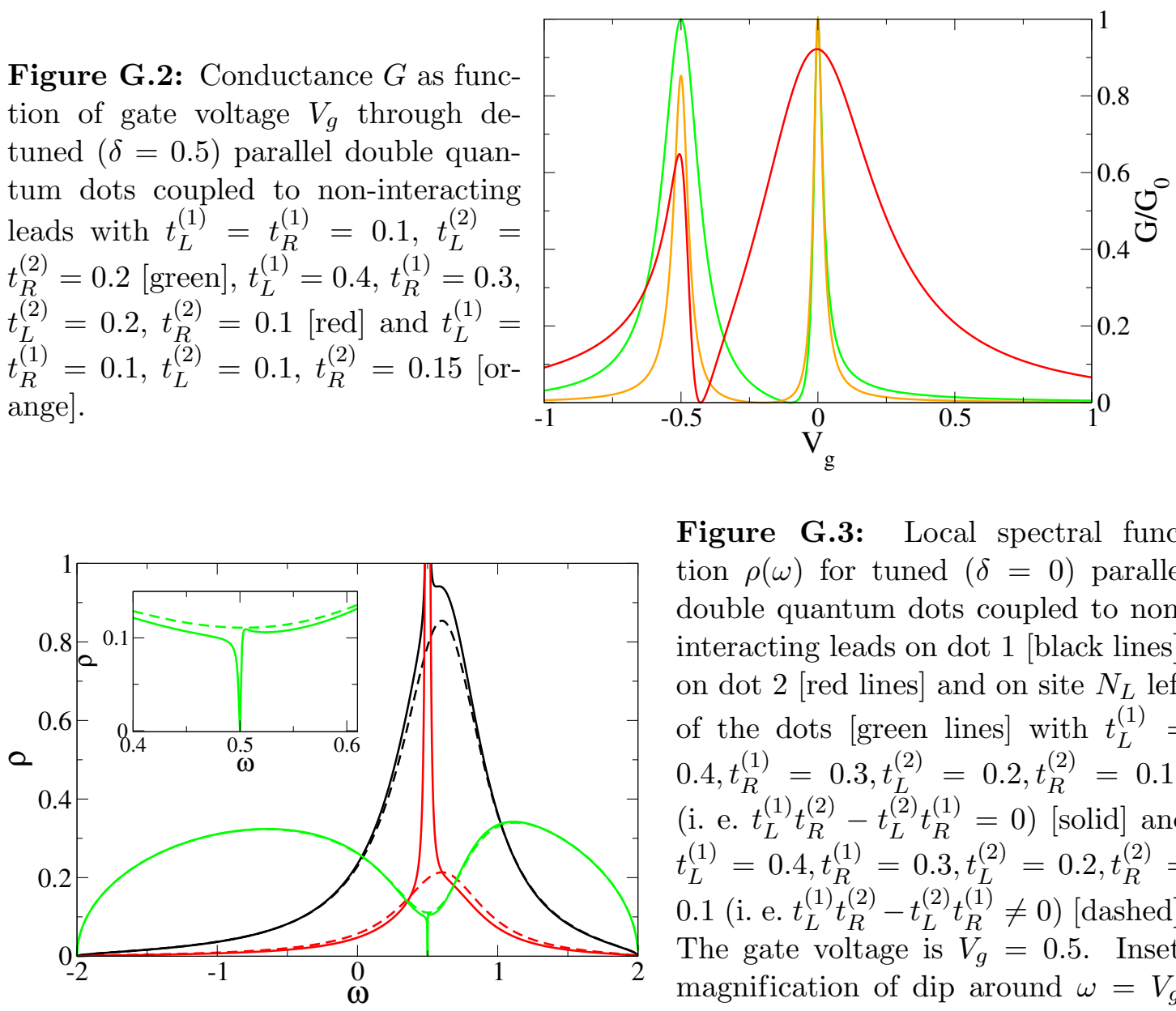

Figure G.3: Local spectral function $\rho(\omega)$ for tuned $(\delta=0)$ parallel double quantum dots coupled to noninteracting leads on dot 1 [black lines], on dot 2 [red lines] and on site $N_{L}$ left of the dots [green lines] with $t_{L}^{(1)}=$ $0.4, t_{R}^{(1)}=0.3, t_{L}^{(2)}=0.2, t_{R}^{(2)}=0.15$ (i. e. $t_{L}^{(1)} t_{R}^{(2)}-t_{L}^{(2)} t_{R}^{(1)}=0$ ) [solid] and $t_{L}^{(1)}=0.4, t_{R}^{(1)}=0.3, t_{L}^{(2)}=0.2, t_{R}^{(2)}=$ 0.1 (i. e. $t_{L}^{(1)} t_{R}^{(2)}-t_{L}^{(2)} t_{R}^{(1)} \neq 0$ ) [dashed]. The gate voltage is $V_{g}=0.5$. Inset: magnification of dip around $\omega=V_{g}$, coloring as in main plot.

1/2). The associated $\delta$-peaks are not included in fig. G.3. For $t_{L}^{(1)} t_{R}^{(2)}-t_{L}^{(2)} t_{R}^{(1)} \neq 0$, the bound states merge with the band, leading to the two sharp peaks at $\omega=V_{g}$ [solid lines]. Their sharpness decreases with increasing $\left|t_{L}^{(1)} t_{R}^{(2)}-t_{L}^{(2)} t_{R}^{(1)}\right|$. Note that for the parameters of the solid curves $\left|t_{L}^{(1)} t_{R}^{(2)}-t_{L}^{(2)} t_{R}^{(1)}\right|=0.02$ holds, which leads to the very sharp peak structure.

The local spectral function next to the dots [green lines in fig. G.3, on site $N_{L}$, but similar on $N_{R}$ ] generically shows a dip at $\omega=V_{g}$, whose width changes similar to the width of the Lorentz peaks on the dots. For $t_{L}^{(1)} t_{R}^{(2)}-t_{L}^{(2)} t_{R}^{(1)} \neq 0, \rho\left(\omega=V_{g}\right)=0$ in an additional sharp dip at $\omega=V_{g}$. The width of this dip is determined by $\left|t_{L}^{(1)} t_{R}^{(2)}-t_{L}^{(2)} t_{R}^{(1)}\right|$ as the width of the sharp additional peaks on the dots. This so called Fano anti-resonance, cf. [Fan61], induces the vanishing conductance at $V_{g}=0$ [red line in fig. G.1] as the transmission probability for an electron to tunnel from the left to the right of the dot structure is (at $T=0)$ proportional to the product of the local spectral functions at $\omega=0$ on the left and right of the structure (Fermi's Golden rule, as used in chapter 4 and section 5.3.

Fig. G.4 exemplifies the local spectral functions on dot 1 [black line], on dot 2 [red line] and on site $N_{L}$ next to the dots [green line] for detuned dots with 
Figure G.4: Local spectral function $\rho(\omega)$ for detuned $(\delta=0.5)$ parallel double quantum dots coupled to noninteracting leads on dot 1 [black], on dot 2 [red] and on site $N_{L}$ left of the dots [green] with $t_{L}^{(1)}=0.4, t_{R}^{(1)}=$ $0.3, t_{L}^{(2)}=0.2, t_{R}^{(2)}=0.1$. The gate voltage is $V_{g}=-0.25$. Inset: overview over entire local spectral functions, coloring as in main plot.

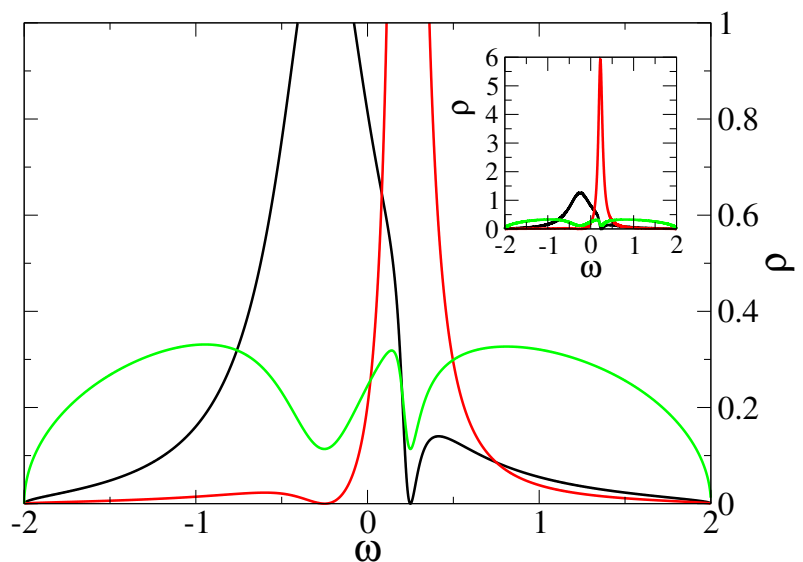

$\delta=0.5$ and $V_{g}=-0.25$. With detuning, no bound states exist and no Fano antiresonance appears, such that the difference between the cases $t_{L}^{(1)} t_{R}^{(2)}-t_{L}^{(2)} t_{R}^{(1)} \neq 0$ and $t_{L}^{(1)} t_{R}^{(2)}-t_{L}^{(2)} t_{R}^{(1)}=0$ is no longer significant. Each dot has a pronounced peak in the local spectral function around its eigenenergy at zero couplings and a vanishing local spectral function at the peak position of the other dot. The width and height of these peaks is determined by the strength of the coupling to the leads. The local spectral function next to the dot (exemplified for $N_{L}$ in the figure but similar on $N_{R}$ ) exhibits two dips, one at each peak position of the dots. The width of these dips changes with the width of the respective peak. 
list of references 



\section{in alphabetical order of labels}

[AEM $\left.{ }^{+} 04\right] \quad$ Andergassen S., Enss T., Meden V., Metzner W., Schollwöck U. and Schönhammer K., Functional renormalization group for Luttinger liquids with impurities. Physical Review B, 70, 075102 (2004).

[AEM06a] Andergassen S., Enss T. and Meden V., Kondo physics in transport through a quantum dot with Luttinger liquid leads. Physical Review B, 73, 153308 (2006).

$\left[\mathrm{AEM}^{+}\right.$06b] Andergassen S., Enss T., Meden V., Metzner W., Schollwöck U. and Schönhammer K., Renormalization-group analysis of the onedimensional extended Hubbard model with a single impurity. Physical Review B, 73, 045125 (2006).

[AG69] Anderson P.W. and Gefen Y., Exact results in the Kondo problem: equivalence to a classical one-dimensional Coulomb gas. Physical Review Letters, 23, 89 (1969).

[AM76] Ashcroft N.W. and Mermin M.D., Solid state physics (Thomson Learning, New York, 1976), 1st edition.

[And70] Anderson P.W., A poor man's derivation of scaling laws for the Kondo problem. Journal of Physics C: Solid State Physics, 3, 2436 (1970).

[And06] Andergassen S., Functional renormalization-group analysis of Luttinger liquids with impurities, thesis, Stuttgart (2006).

[ANS08] Arrachea L., Naón C. and Salvay M., Voltage profile and four-terminal resistance of an interacting wire. Physical Review B, 77, 233105 (2008).

[AR82] Apel W. and Rice T.M., Combined effect of disorder and interaction on the conductance of a one-dimensional fermion system. Physical Review B, 26, 7063 (1982).

[AS72] Abramowitz M. and Stegun I.A., Handbook of mathematical functions (Dover Publications, New York, 1972), 9th edition.

[AW09] Aristov D.N. and Wölfle P., Conductance through a potential barrier embedded in a Luttinger liquid: nonuniversal scaling at strong coupling. Physical Review B, 80, 045109 (2009). 
[BCL $\left.{ }^{+} 99\right] \quad$ Bockrath M., Cobden D.H., Lu J., Rinzler A.G., Smalley R.E., Balents L. and McEuen P.L., Luttinger-liquid behavior in carbon nanotubes. Nature, 397, 598 (1999).

[BD04] Baeriswyl D. and Degiorgi L. (editors), Strong interactions in low dimensions (Kluwer Academic, New York, 2004), 1st edition.

[Bet31] Bethe H., Zur Theorie der Metalle: I. Eigenwerte und Eigenfunktionen der linearen Atomkette. Zeitschrift für Physik, 71, 205 (1931).

[BF04] Bruus H. and Flensberg K., Many-body quantum theory in condensed matter physics (Oxford University Press, Oxford, 2004), 1st edition.

[BGM $\left.{ }^{+} 05\right]$ Biercuk M.J., Garaj S., Mason N., Chow J.M. and Marcus C.M., Gate-defined quantum dots on carbon nanotubes. Nano Letters, 5, 1267 (2005).

[BILP85] Büttiker M., Imry Y., Landauer R. and Pinhas S., Generalized manychannel conductance formula with application to small rings. Physical Review B, 31, 6207 (1985).

[BM09] Birkholz J.E. and Meden V., Spin-polarized currents through interacting quantum wires with nonmagnetic leads. Physical Review B, 79, 085420 (2009).

[BTSMS05a] Barnabé-Thérault X., Sedeki A., Meden V. and Schönhammer K., Junction of Three Quantum Wires: Restoring Time-Reversal Symmetry by Interaction. Physical Review Letters, 94, 136405 (2005).

[BTSMS05b] Barnabé-Thérault X., Sedeki A., Meden V. and Schönhammer K., Junctions of one-dimensional quantum wires: Correlation effects in transport. Physical Review B, 71, 205327 (2005).

[BZAS99] Berman D., Zhitenev N.B., Ashoori R.C. and Shayegan M., Observation of quantum fluctuations of charge on a quantum dot. Physical Review Letters, 82, 161 (1999).

[Cal74] Callaway J., Quantum theory of the solid state (Academic Press, London, 1974), 1st edition.

[CBR07] Charlier J.C., Blase X. and Roche S., Electronic and transport properties of nanotubes. Reviews of Modern Physics, 79, 677 (2007).

[CGH ${ }^{+}$96] Corless R.M., Gonnet G.H., Hare D.E.G., Jeffrey D.J. and Knuth D.E., On the Lambert $W$ function. Advances in Computational Mathematics, 5, 329 (1996).

[Cha03] Chang A.M., Chiral Luttinger liquid at the fractional quantum Hall edge. Reviews of Modern Physics, 75, 1449 (2003). 
[CJF92] Callan Jr. C.G. and Freed D., Phase diagram of the dissipative Hofstatder model. Nuclear Physics B, 374, 543 (1992).

[CJKMY95] Callan Jr. C.G., Klebanov I.R., Maldacena J.M. and Yegulalp A., Magnetic fields and fractional statistics in boundary conformal field theory. Nuclear Physics B, 443, 444 (1995).

[CJM05] Cornean H.D., Jensen A. and Moldoveanu V., A rigorous proof of the Landauer-Büttiker formula. Journal of Mathematical Physics, 46, 042106 (2005).

[COA03] Chamon C., Oshikawa M. and Affleck I., Junctions of three wires and the dissipative Hofstadter model. Physical Review Letters, 91, 206403 (2003).

[Coj06] Cojocaru S., Green's function of a finite chain and the discrete Fourier transform. International Journal of Modern Physics B, 20, 593 (2006).

[Coj07] Cojocaru S., Green's functions on finite lattices and their connection to the infinite lattice limit (2007), arXiv:0709.0917v2.

[COK98] Cronenwett S.M., Oosterkamp T.H. and Kouwenhoven L.P., A tunable Kondo effect in quantum dots. Science, 281, 540 (1998).

[CPW96] Chang A.M., Pfeiffer L.N. and West K.W., Observation of chiral Luttinger behavior in electron tunneling into fractional quantum Hall edges. Physical Review Letters, 77, 2538 (1996).

[CPW98] Chang A.M., Pfeiffer L.N. and West K.W., Apparent $g=1 / 2$ chiral Luttinger liquid at the edge of the $\nu=1 / 2$ compressible composite Fermion liquid. Physica B, 249, 383 (1998).

[CTDL77] Cohen-Tannoudji C., Diu B. and Laloë F., Quantum mechanics (John Wiley \& Sons, New York, 1977), 1st edition.

[CW93] Chamon C. and Wen X.G., Resonant tunneling in the fractional quantum hall regime. Physical Review B, 47, 3827 (1993).

[Dat95] Datta S., Electronic transport in mesoscopic systems (Cambridge University Press, New York, 1995), 1st edition.

[DC96] Dupuis N. and Chitov G.Y., Renormalization-group approach to Fermi-liquid theory. Physical Review B, 54, 3040 (1996).

[DHL07] Dupas C., Houdy P. and Lahmani M., Nanoscience: Nanotechnologies and nanophysics (Springer Verlag, Berlin, 2007), 1st edition. 
[DKC $\left.{ }^{+} 08\right]$ Danneau R., Klochan O., Clarke W.R., Ho L.H., Micolich A.P., Simmons M.Y., Hamilton A.R., Pepper M. and Ritchie D.A., 0.7 structure and zero bias anomaly in ballistic hole quantum wires. Physical Review Letters, 100, 016403 (2008).

$\left[\mathrm{DLW}^{+}\right.$99] Duncan D.S., Livermore C., Westervelt R.M., Maranowski K.D. and Gossard A.C., Direct measurement of the destruction of charge quantization in a single-electron box. Applied Physics Letters, 74, 1045 (1999).

[DRS04] Das S., Rao S. and Sen D., Renormalization group study of the conductances of interacting quantum wire systems with different geometries. Physical Review B, 70, 085318 (2004).

[Eco79] Economou E.N., Green's functions in quantum physics (Springer Verlag, Berlin, 1979), 1st edition.

[EG96] Egger R. and Grabert H., Voltage-biased quantum wire with impurities. Physical Review Letters, 77, 538 (1996).

[EG97] Egger R. and Gogolin A.O., Effective low-energy theory for correlated carbon nanotubes. Physical Review Letters, 79, 5082 (1997).

[EG98] Egger R. and Gogolin A.O., Correlated transport and non-Fermi-liquid behavior in single-wall carbon nanotubes. European Physical Journal B, 3, 281 (1998).

[EH98] Egger R. and H. G., Applying voltage sources to a Luttinger liquid with arbitrary transmission. Physical Review B, 58, 10761 (1998).

$\left[\mathrm{EMA}^{+} 05\right]$ Enss T., Meden V., Andergassen S., Barnabé-Thériault X., Metzner W. and Schönhammer K., Impurity and correlation effects on transport in one-dimensional quantum wires. Physical Review B, 71, $155401(2005)$.

[EMOT53] Erdélyi A., Magnus W., Oberhettinger F. and Tricomi F.G., Higher Transcendental Functions (McGraw-Hill Book Company, New York, 1953), 1st edition.

[Ens05] Enss T., Renormalization, conservation laws and transport in correlated electron systems, thesis, Stuttgart (2005).

[Fan61] Fano U., Effects of Configuration Interaction on Intensities and Phase Shifts. Physical Review, 124, 1866 (1961).

[Fes58] Feshbach H., A unified theory of nuclear reactions. Annals of Physics, 5, 357 (1958). 
[Fes62] Feshbach H., A unified theory of nuclear reactions. II. Annals of Physics, 19, 287 (1962).

[FG95] Fabrizio M. and Gogolin A.O., Interacting one-dimensional electron gas with open boundaries. Physical Review B, 51, 17827 (1995).

[FM02] Furusaki A. and Matveev K.A., Occupation of a resonant level coupled to a chiral Luttinger liquid. Physical Review Letters, 88, 226404 (2002).

[FN93] Furusaki A. and Nagaosa N., Resonant tunneling in a Luttinger liquid. Physical Review B, 47, 3827 (1993).

[FN94] Furusaki A. and Nagaosa N., Kondo effect in a Tomonaga-Luttinger liquid. Physical Review Letters, 72, 892 (1994).

[FSP ${ }^{+}$93] Field M., Smith C.G., Pepper M., Ritchie D.A., Frost J.E.F., Jones G.A.C. and Hasko D.G., Measurements of Coulomb blockade with a noninvasive voltage probe. Physics Review Letters, 70, 1311 (1993).

[Fur98] Furusaki A., Resonant tunneling through a quantum dot weakly coupled to quantum wires or quantum Hall edge states. Physical Review B, 57, 7141 (1998).

[Fur05] Furusaki A., Kondo problems in Tomonaga-Luttinger liquids. Journal of the Physical Society of Japan, 74, 73 (2005).

[GAD03] Gweon G.H., Allen J.W. and Denlinger J.D., Generalized spectral signatures of electron fractionalization in quasi-one-and two-dimensional molybdenum bronzes and superconducting cuprates. Physical Review B, 68, 195117 (2003).

[GB09] Goldstein M. and Berkovits R., Duality between the different geometries of a resonant level in a Luttinger liquid (2009), arXiv:0907.0424.

$\left[\mathrm{GDO}^{+} 02\right]$ Gweon G.H., Denlinger J.D., Olson C.G., Höchst H., Marcus J. and Schlenker C., Photoemission view of electron fractionalization in quasi-one-dimensional metal $\mathrm{Li}_{0.9} \mathrm{Mo}_{6} \mathrm{O}_{17}$. Physica $\mathrm{B}, \mathbf{3 1 2 - 3 1 3}, 584$ (2002).

[Gia04] Giamarchi T., Quantum physics in one dimension (Oxford University Press, Oxford, 2004), 1st edition.

[GKE ${ }^{+}$04] Gao B., Komnik A., Egger R., Glattli D.C. and Bachtold A., Evidence for Luttinger-liquid behavior in crossed metallic single-wall nanotubes. Physical Review Letters, 92, 216804 (2004).

[GM09] Grap S. and Meden V., Renormalization group study of Luttinger liquids with boundaries. Physical Review B, 80, 193106 (2009). 
[Gol93] Goldenfeld N., Lectures on phase transitions and the renormalization group (Addison-Wesley, New York, 1993), 3rd edition.

[GV05] Giuliani G.F. and Vignale G., Quantum theory of the electron liquid (Cambridge University Press, Cambridge, 2005), 1st edition.

[GWB09] Goldstein M., Weiss Y. and Berkovits R., Interacting resonant level coupled to a Luttinger liquid: Universality of thermodynamic properties. Europhysics Letters, 86, 67012 (2009).

[Hal80] Haldane F.D.M., General relation of correlation exponents and spectral properties of one-dimensional Fermi systems: application to the anisotropic $S=\frac{1}{2}$ Heisenberg chain. Physical Review Letters, 45, 1358 (1980).

[Hal81a] Haldane F.D.M., Demonstration of the "Luttinger liquid" character of Bethe-ansatz-soluble models of 1-d quantum fluids. Physics Letters, 81A, 153 (1981).

[Hal81b] Haldane F.D.M., "Luttinger liquid theory" of one-dimensional quantum fluids: I. Properties of the Luttinger model and their extension to the general 1D interacting spinless Fermi gas. Journal of Physics C, 14, 2585 (1981).

[Har01] Harris P.J.F., Carbon nanotubes and related structures: New materials for the twenty-first century (Cambridge University Press, Cambridge, 2001), 2nd edition.

[HE04] Hügle S. and Egger R., Resonant tunneling in a Luttinger liquid for arbitrary barrier transmission. Europhysics Letters, 66, 565 (2004).

[Hei03] Heinzel T. (editor), Mesoscopic electronics in solid state nanostructures (Wiley-VCH, Weinheim, 2003), 1st edition.

$\left[\mathrm{HKP}^{+} 07\right]$ Hanson R., Kouwenhoven L.P., Peta J.R., Tarucha S. and Vandersypen L.M.K., Spin in few-electron quantum dots. Reviews of Modern Physics, 79, 1217 (2007).

[HMPS04] Hedden R., Meden V., Pruschke T. and Schönhammer K., Functional renormalization group approach to zero-dimensional interacting systems. Journal of Physics: Condensed Matter, 16, 5279 (2004).

[IG09a] Imambekov A. and Glazman L.I., Phenomenology of one-dimensional quantum liquids beyond the low-energy limit. Physical Review Letters, 102, 126405 (2009).

[IG09b] Imambekov A. and Glazman L.I., Universal theory of nonlinear Luttinger liquids. Science, 323, 228 (2009). 
[IL99] Imry Y. and Landauer R., Conductance viewed as transmission. Reviews of Modern Physics, 71, S306 (1999).

[Imr97] Imry Y., Introduction to mesoscopic physics (Oxford University Press, New York, 1997), 1st edition.

[JGRHL06] Jørgensen H.I., Grove-Rasmussen K., Hauptmann J.R. and Lindelof P.E., Single wall carbon nanotubes double quantum dot. Applied Physics Letters, 89, 232113 (2006).

[JMS06] Janzen K., Meden V. and Schönhammer K., Influence of the contacts on the conductance of interacting quantum wires. Physical Review B, 74, 085301 (2006).

[JMS07] Jakobs S.G., Meden V. and Schoeller H., Nonequilibrium functional renormalization group for interacting quantum systems. Physical Review Letters, 99, 150603 (2007).

[JMSE07] Jakobs S.G., Meden V., Schoeller H. and Enss T., Temperatureinduced phase averaging in one-dimensional mesoscopic systems. Physical Review B, 75, 035126 (2007).

[JW28] Jordan P. and Wigner E., Über das Paulische Äquivalenzverbot. Zeitschrift für Physik, 47, 631 (1928).

[Kaw09] Kawaguchi S., Nonmonotonic behaviors of Fano factor in double quantum dot connected with Luttinger liquid electrodes. Journal of Physics: Condensed Matter, 21, 395303 (2009).

[KBF97] Kane C., Balents L. and Fisher M.P.A., Coulomb interactions and mesoscopic effects in carbon nanotubes. Physical Review Letters, 79, 5086 (1997).

[KCSK02] Kleimann T., Cavaliere F., Sassetti M. and Kramer B., Theory of measuring the Luttinger $g$ of a one-dimensional quantum dot. Physical Review B, 66, 165311 (2002).

[Kel76] Keller H. (editor), Chemistry and physics of one-dimensional metals, volume 25 of NATO Advanced study institutes series: Series B, Physics (Plenum Press, New York, 1976).

[KEM06] Karrasch C., Enss T. and Meden V., A novel approach to transport through correlated quantum dots. Physical Review B, 73, 235337 (2006).

[KF92a] Kane C.L. and Fisher M.P.A., Resonant tunneling in an interacting one-dimensional electron gas. Physical Review B, 46, 7268 (1992). 
[KF92b] Kane C.L. and Fisher M.P.A., Transmission through barriers and resonant tunneling in an interacting one-dimensional electron gas. Physical Review B, 46, 15233 (1992).

[KF92c] Kane C.L. and Fisher M.P.A., Transport in a one-channel Luttinger liquid. Physical Review Letters, 68, 1220 (1992).

[KHM98] Karbach M., Hu K. and Müller G., Introduction to the Bethe Ansatz II. Computers in Physics, 12, 565 (1998).

[KHM00] Karbach M., Hu K. and Müller G., Introduction to the Bethe Ansatz III (2000), arXiv:cond-mat/0008018.

[KHP $\left.{ }^{+} 08\right] \quad$ Karrasch C., Hedden R., Peters R., Pruschke T., Schönhammer K. and Meden V., A finite-frequency functional $R G$ approach to the single impurity Anderson model. Journal of Physics: Condensed Matter, 20, 345205 (2008).

$\left[\mathrm{KHW}^{+} 07\right]$ Karrasch C., Hecht T., Weichselbaum A., von Delft J., Oreg Y. and Meden V., Phase lapses in transmission through interacting two-level quantum dots. New Journal of Physics, 9, 123 (2007).

[KM97] Karbach M. and Müller G., Introduction to the Bethe Ansatz I. Computers in Physics, 11, 36 (1997).

[KMHM07] Klironomos A.D., Meyer J.S., Hikihara T. and Matveev K.A., Spin coupling in zigzag Wigner crystals. Physical Review B, 76, 075302 (2007).

[KROY07] Kim N.Y., Recher P., Oliver W.D. and Yamamoto Y., TomonagaLuttinger liquid features in ballistic single-walled carbon nanotubes: conductance and shot noise. Physical Review Letters, 99, 156803 (2007).

[KSAEW07] Kashcheyevs V., Schiller A., Aharony A. and Entin-Wohlman O., Unified description of phase lapses, population inversion, and correlationinduced resonances in double quantum dots. Physical Review B, 75, 115313 (2007).

[Lan70] Landauer R., Electrical resistance of disordered one-dimensional lattices. Philosophical Magazine, 21, 863 (1970).

[Lan96] Landauer R., Spatial variation of currents and fields due to localized scatteres in metallic conduction (and comment). Journal of Mathematical Physics, 37, 5259 (1996).

[LM66] Lieb E.H. and Mattis D.C., Mathematical physics in one dimension (Academic Press, New York, 1966), 1st edition. 
[LP74] Luther A. and Peschel I., Fluctuation conductivity and lattice stability in one dimension. Physical Review Letters, 32, 992 (1974).

[LRS02] Lal S., Rao S. and Sen D., Junction of several weakly interacting quantum wires: A renormalization group study. Physical Review B, 66, 165327 (2002).

[Lut63] Luttinger J.M., An exactly soluble model of a many-fermion system. Journal of Mathematical Physics, 4, 1154 (1963).

[LYY08] Lerner I.V., Yudson V.I. and Yurkevich I.V., Quantum wire hybridized with a single-level impurity. Physical Review Letters, 100, 256805 (2008).

[MAE $\left.{ }^{+} 08\right] \quad$ Meden V., Andergassen S., Enss T., Schoeller H. and Schönhammer K., Fermionic renormalization group methods for transport through inhomogeneous Luttinger liquids. New Journal of Physics, 10, 045012 (2008).

[Mah00] Mahan G.D., Many-particle physics (Kluwer Academic, New York, 2000), 3rd edition.

$\left[\mathrm{MAM}^{+}\right.$03] Meden V., Andergassen S., Metzner W., Schollwöck U. and Schönhammer K., Scaling of the conductance in a quantum wire. Europhysics Letters, 64, 769 (2003).

[Mas95] Maslov D.L., Transport through dirty Luttinger liquids connected to reservoirs. Physical Review B, 52, R14368 (1995).

[MBM04] Mason N., Biercuk M.J. and Marcus C.M., Local gate control of a carbon nanotube double quantum dot. Science, 303, 655 (2004).

[Med] Meden V., Lecture Notes, URL http://www.theorie.physik.uni -goettingen.de/\$ $\backslash$ sim\$meden/funRG/

[Med96] Meden V., Spektrale Eigenschaften niedrigdimensionaler korrelierter Elektronensysteme, thesis, Göttingen (1996).

[ML65] Mattis D.C. and Lieb E.H., Exact solution of a many-fermion system and its associated boson field. Journal of Mathematical Physics, 6, 304 (1965).

[MM06] Meden V. and Marquardt F., Correlation-induced resonances in transport through coupled quantum dots. Physical Review Letters, 96, 146801 (2006).

$\left[\mathrm{MMS}^{+} 00\right]$ Meden V., Metzner W., Schollwöck U., Schneider O., Stauber T. and Schönhammer K., Luttinger liquids with boundaries: Power laws and energy scales. European Journal of Physics, 16, 631 (2000). 
[MMSS02a] Meden V., Metzner W., Schollwöck U. and Schönhammer K., Scaling behavior of impurities in mesoscopic Luttinger liquids. Physical Review B, 65, 045318 (2002).

[MMSS02b] Meden V., Metzner W., Schollwöck U. and Schönhammer K., A single impurity in a Luttinger liquid: How it "Cuts" the chain. Journal of Low Temperature Physics, 126, 1147 (2002).

[Mor11] Morris T.R., The exact renormalisation and approximate solutions. International Journal of Modern Physics A, 9, 1994 (2411).

[MS92] Meden V. and Schönhammer, Spectral functions of the TomonagaLuttinger model. Physical Review B, 46, 15753 (1992).

[MS95] Maslov D.L. and Stone M., Landauer conductance of Luttinger liquids with leads. Physical Review B, 52, R5539 (1995).

[MS03] Meden V. and Schollwöck U., Conductance of interacting nanowires. Physical Review B, 67, 193303 (2003).

[MYG93] Matveev K.A., Yue D. and Glazman L.I., Tunneling in onedimensional non-Luttinger electron liquid. Physical Review Letters, 71, 3351 (1993).

[NB09] Nazarov Y.V. and Blanter Y.M., Quantum transport (Cambridge University Press, New York, 2009), 1st edition.

[NFLL04] Nayak C., Fisher M.P.A., Ludwig A.W.W. and Lin H.H., Resonant multilead point-contact tunneling. Physical Review B, 70, 195115 (2004).

[NG03] Nazarov Y.V. and Glazman L.I., Resonant tunneling of interacting electrons in a one-dimensional wire. Physical Review Letters, 91, 126804 (2003).

[NO98] Negele J.W. and Orland H., Quantum many-particle systems (Westview Press, Aspen, 1998), 1st edition.

[NTC06] Nemec N., Tománek D. and Cuniberti G., Contact dependence of carrier injection in carbon naotubes: an ab-initio study. Physical Review Letters, 96, 076802 (2006).

[NTC08] Nemec N., Tománek D. and Cuniberti G., Modeling extended contacts for nanotube and graphene devices. Physical Review B, 77, 125420 (2008).

[Ogu99] Oguri A., Transport through a finite Hubbard chain connected to reservoirs. Physical Review B, 59, 12240 (1999). 
[Ogu01] Oguri A., Transmission probability for interacting electrons connected to reservoirs. Journal of the Physical Society of Japan, 70, 2666 (2001).

[PdYD00] Postma H.W.C., de Jonge M., Yao Z. and Dekker C., Electrical transport through carbon nanotube junctions created by mechanical manipulation. Physical Review B, 62, R10653 (2000).

[PG03] Polyakov D.G. and Gornyi I.V., Transport of interacting electrons through a double barrier in quantum wires. Physical Review B, 68, 035421 (2003).

[Pin61] Pines D., The many-body problem (W. A. Benjamin, New York, 1961), 1st edition.

[Pon95] Ponomarenko V.V., Renormalization of the one-dimensional conductance in the Luttinger-liquid model. Physical Review B, 52, R8666 (1995).

[PPJL $\left.{ }^{+} 03\right]$ Palacios J.J., Pérez-Jiménez A.J., Louis E., SanFabián E. and Vergés J.A., First-principles phase-coherent transport in metallic nanotubes with realistic contacts. Physical Review Letters, 90, 106801 (2003).

[PTVF07] Press W.H., Teukolsky S.A., Vetterling W.T. and Flannery B.P., Numerical Recipes (Cambridge University Press, New York, 2007), 3rd edition.

$\left[\mathrm{PTY}^{+} 01\right]$ Postma H.W.C., Teepen T., Yao Z., Grifoni M. and Dekker C., Carbon nanotube single-electron transistors at room temperature. Science, 293, 76 (2001).

[Qua08] Quandt A., Ab-initio approach to the many-electron problem, in Computational Many Particle Physics (edited by H. Fehske, R. Schneider and A. Weiße) (Springer, Berlin, 2008), 1st edition.

[RS04] Rao S. and Sen D., Tunneling through two resonant levels: Fixed points and conductances. Physical Review B, 70, 195115 (2004).

$\left[\right.$ RWD $\left.^{+} 00\right] \quad$ Rother M., Wegscheider W., Deutschmann R.A., Bichler M. and Abstreiter G., Evidence of Luttinger liquid behavior in GaAs/AlGaAs quantum wires. Physica E, 6, 551 (2000).

[Saf97] Safi I., Resonance in a Tomonaga-Luttinger liquid. Physical Review B, 56, R12691 (1997).

[Sch97] Schönhammer K., Interacting fermions in one dimension: the Tomonaga Luttinger model (1997), arXiv:cond-mat/9710330. 
[Sch03] Schönhammer K., Luttinger Liquids: the basic concepts, in Strong interactions in low dimensions (edited by D. Baeriswyl and L. Degiorgi) (Kluwer Academic, New York, 2003), 1st edition.

[Sch05] Schollwöck U., The density-matrix renormalization group. Reviews of Modern Physics, 77, 259 (2005).

[SGK09] Steele G.A., Gotz G. and Kouwenhoven L.P., Tunable few-electron double quanutm dots and Klein tunnelling in ultra-clean carbon nanotubes. Nature Nanotechnology, 4, 363 (2009).

$\left[\mathrm{SGT}^{+} 08\right] \quad$ Sfigakis F., Graham A.C., Thomas K.J., Pepper M., Ford C.J.B. and Ritchie D.A., Spin effects in one-dimensional systems. Journal of Physics: Condensed Matter, 20, 164213 (2008).

[SH01] Salmhofer M. and Honerkamp C., Fermionic renormalization group flows. Progress of Theoretical Physics, 105, 1 (2001).

[Sha94] Shankar R., Renormalization-group approach to interacting fermions. Reviews of Modern Physics, 66, 129 (1994).

[S197] Schiller A. and Ingersent K., Renormalization-group study of a magnetic impurity in a Luttinger liquid. Europhysics Letters, 39, 645 (1997).

[Sit71] Sitenko A.G., Lectures in scattering theory (Pergamon Press, Oxford, 1971), 1st edition.

[SK04] Schwarz H.R. and Köckler N., Numerische Mathematik (Teubner Verlag, Wiesbaden, 2004), 1st edition.

$\left[\mathrm{SMB}^{+}\right.$06] Sapmaz S., Meyer C., Beliczynski P., Jarillo-Herrero P. and Kouwenhoven L.P., Excited state spectroscopy in carbon nanotube double quantum dots. Nano Letters, 6, 1350 (2006).

[Sól79] Sólynom J., The Fermi gas model of one-dimensional conductors. Advances in Physics, 28, 201 (1979).

[SRI ${ }^{+}$04] Schleser R., Ruh E., Ihn T., Ensslin K., Driscoll D.C. and Gossard A.C., Time-resolved detection of individual electrons in a quantum dot. Applied Physics Letters, 85, 2005 (2004).

[SS95] Safi I. and Schulz H.J., Transport in an inhomogeneous interacting one-dimensional system. Physical Review B, 52, R17040 (1995).

[SWGB05] Sade M., Weiss Y., Goldstein M. and Berkovits R., Level coupled to a one-dimensional interacting reservoir: A density matrix renormalization group study. Physical Review B, 71, 153301 (2005). 
[Tak99] Takahashi M., Thermodynamics of one-dimensional solvable models (Cambridge University Press, Cambridge, 1999), 1st edition.

[TDD $\left.{ }^{+} 97\right] \quad$ Tans S.J., Devoret M.H., Dai H., Thess A., Smalley R.E., Geerligs L.J. and Dekker C., Individual single-wall carbon nanotubes as quantum wires. Nature, 386, 474 (1997).

[TEG02] Trauzettel B., Egger R. and Grabert H., Coulomb drag shot noise in coupled Luttinger liquids. Physical Review Letters, 99, 116401 (2002).

[TEG05] Thorwart M., Egger R. and Grifoni M., Correlated sequential tunneling through a double barrier for interacting one-dimensional electrons. Physical Review B, 72, 035330 (2005).

[TGC $\left.{ }^{+} 02\right]$ Thorwart M., Grifoni M., Cuniberti G., Postma H.W.C. and C. D., Correlated tunneling in intramolecular carbon nanotube quantum dots. Physical Review Letters, 89, 196402 (2002).

[THS95] Tarucha S., Honda T. and Saku T., Reduction of quantizes conductance at low temperatures observed in 2 to $10 \mu \mathrm{m}$-long quantum wires. Solid State Communications, 94, 413 (1995).

[Tom50] Tomonaga S., Remarks on Bloch's method of sound waves applied to many-fermion problems. Progress of Theoretical Physics, 5, 544 (1950).

[TW83] Tsvelick A.M. and Wiegmann P.B., Exact results in the theory of magnetic alloys. Advances in Physics, 32, 453 (1983).

[vDS98] von Delft J. and Schoeller H., Bosonization for beginners - refermionization for experts. Annalen der Physik (Leipzig), 7, 225 (1998).

[Voi94] Voit J., One-dimensional Fermi liquids. Reports on Progress in Physics, 57, 977 (1994).

[Wäc06] Wächter P., Indirekte Wechselwirkung zwischen Verunreinigungen in Luttingerflüssigkeiten, diploma thesis, University of Göttingen (2006).

[Wet01] Wetterich C., Exact evolution potential for the effective potential. Progress of Theoretical Physics, 105, 1 (2001).

[Whi92] White S.R., Density matrix formulation for quantum renormalization groups. Physical Review Letters, 69, 2863 (1992).

[Wil75] Wilson K.G., The renormalization group: Critical phenomena and the Kondo problem. Reviews of Modern Physics, 773, 47 (1975).

[WK74] Wilson K.G. and Kogut J., The renormalization group and the $\varepsilon$ expansion. Physics Reports, 12, 75 (1974). 
[WMS07a] Wächter P., Meden V. and Schönhammer K., Charging of a quantum dot coupled to Luttinger liquid leads. Physical Review B, 76, 125316 (2007).

[WMS07b] Wächter P., Meden V. and Schönhammer K., Indirect forces between impurities in one-dimensional quantum liquids. Physical Review B, 76, 045123 (2007).

[WMS09] Wächter P., Meden V. and Schönhammer K., Coupling-geometryinduced temperature scales in the conductance of Luttinger liquid wires. Journal of Physics: Condensed Matter, 21, 215608 (2009).

[WQN $\left.{ }^{+} 07\right]$ Wu F., Queipo P., Nasibulin A., Tsuneta T., Wang T.H., Kauppinen E. and Hakonen P.J., Shot noise with interaction effects in singelwalled carbon nanotubes. Physical Review Letters, 99, 156803 (2007).

[YGM94] Yue D., Glazman L.I. and Matveev K.A., Conduction of a weakly interacting one-dimensional electron gas through a single barrier. Physical Review B, 49, 1966 (1994).

[YPBD99] Yao Z., Postma H.W.C., Balents L. and Dekker C., Carbon nanotube intramolecular junctions. Nature, 402, 273 (1999).

[YY66a] Yang C.N. and Yang C.P., One-dimensional chain of anisotropic spinspin interactions. I. proof of Bethe's hypothesis for ground state in a finite system. Physical Review, 150, 321 (1966).

[YY66b] Yang C.N. and Yang C.P., One-dimensional chain of anisotropic spinspin interactions. II. properties of the ground-state energy per lattice site for an infinite system. Physical Review, 150, 327 (1966).

[YY66c] Yang C.N. and Yang C.P., One-dimensional chain of anisotropic spinspin interactions. III. applications. Physical Review, 151, 258 (1966).

[ZJ89] Zinn-Justin J., Quantum field theory and critical phenomena (Oxford University Press, Oxford, 1989), 1st edition. 


\section{Lebenslauf}

Name: Hans Peter Wächter

Staatsangehörigkeit: deutsch

- 05.06.1981: geboren in Hannover, Niedersachsen

- 06.1987-06.2000: Besuch von Grundschule, Orientierungsstufe und Gymnasium (Wilhelm-Raabe Schule, Hannover)

- 30.06.2000: Erwerb der allgemeinen Hochschulreife

- 10.2001-09.2004: Studium der Physik an der Universität Göttingen

- 17.07.2003: Erwerb des Vordiploms in Physik

- 09.2004-06.2005: Studium der Physik an der Universität von Kalifornien, Santa Barbara

- 07.2005-11.2006: Studium der Physik an der Universität Göttingen

- 09.11.2006: Verleihung des Hochschulgrades „Diplom-Physiker“

- seit 01.2007: Arbeit an der vorliegenden Dissertation bei Prof. Dr. K. Schönhammer

- seit 04.2007: Studium im wirtschaftswissenschaftlichen Zusatzstudiengang für Ingenieure und Naturwissenschaftler der Fernuniversität Hagen

- 31.03.2008: Erwerb des Vordiploms im wirtschaftswissenschaftlichen Zusatzstudiengang für Ingenieure und Naturwissenschaftler 